\title{
GA-A16258
}

UC-77

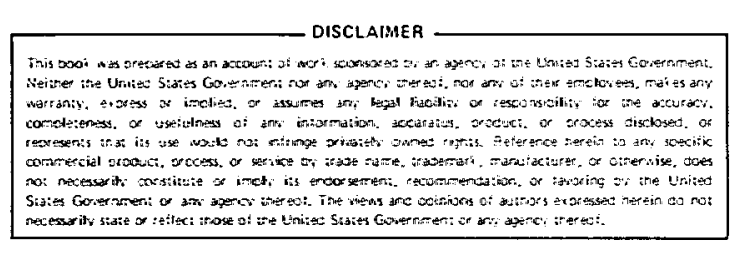

\section{POSTIRRADIATION EXAMINATION AND EVALUATION OF FORT ST. VRAIN FUEL ELEMENT 1-0743}

\author{
by \\ J. J. SAURWEIN, C. M. MILLER, and C. A. YOUNG
}

\author{
Prepared under \\ Contract DE-AT03-76ET35301 \\ for the San Francisco Operations Office \\ Department of Energy
}

\section{GENERAL ATOMIC PROJECT 7400 \\ DATE PUBLISHED: MAY 1981}

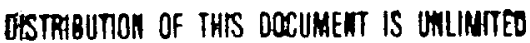

\section{GENERAL ATOMIC COMPANY}




\section{DISCLAIMER}

This report was prepared as an account of work sponsored by an agency of the United States Government. Neither the United States Government nor any agency Thereof, nor any of their employees, makes any warranty, express or implied, or assumes any legal liability or responsibility for the accuracy, completeness, or usefulness of any information, apparatus, product, or process disclosed, or represents that its use would not infringe privately owned rights. Reference herein to any specific commercial product, process, or service by trade name, trademark, manufacturer, or otherwise does not necessarily constitute or imply its endorsement, recommendation, or favoring by the United States Government or any agency thereof. The views and opinions of authors expressed herein do not necessarily state or reflect those of the United States Government or any agency thereof. 


\section{DISCLAIMER}

Portions of this document may be illegible in electronic image products. Images are produced from the best available original document. 


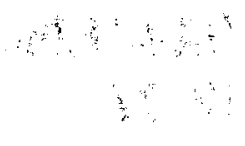

$\bullet$ 
ABSTRACT

Fort St. Vrain (FSV) fuel element 1-0743 was irradiated in core location 17.04.F.06 from July 3, 1976 until February 1, 1979. The element experienced an average fast neutron exposure of about $0.95 \times 10^{25} \mathrm{n} / \mathrm{m}^{2}$ (E > $29 \mathrm{fJ})_{\mathrm{HTGR}}$, a time-and-volume-averaged fuel temperature in the vicinity of $680^{\circ} \mathrm{C}$, fissile and fertile particle burnups of approximately $6.2 \%$ anc. $0.3 \%$, respectively, and a total burnup of $12,210 \mathrm{MWd} /$ tonne. The postirradiation examination of the fuel element was performed as part of the Department of Energy (DOE) sponsored surveillance program for the FSV hightemperature gas-cooled reactor (HTGR). The purpose of the examination was to verify the acceptable performance of the element and to acquire in-pile data for verification of HTGR core design data and methods.

The postirradiation examination revealed that the element was in excellent condition. No cracks were observed on any of the element surfaces. The structural integrity of the fuel rods was good. No evidence of mechanical interaction between the fuel rods and fuel body was observed. The performance of the TRISO fuel particles was excellent. No kernel migration or fission product attack on the SiC coating was detected. As a result of the fabrication process, there was some fuel dispersion in the buffer coating, but it apparently did not detrimentally affect the irradiation performance of the particles. Metallography and fission gas release measurements revealed that there was no in-pile fuel failure.

Calculated irradiation parameters obtained with HTGR design codes were compared with measured data. Radial and axial power distributions, irradiation temperatures, neutron fluences, and fuel burnups were in good agreement with measurements. Calculated fuel rod strains were about a factor of three greater than were observed. In-pile failure of $0.3 \%$ for the $(\mathrm{Th}, \mathrm{U}) \mathrm{C}_{2}$ fissile particles and $0.1 \%$ for the $\mathrm{ThC}_{2}$ fertile particles, primarily due 
to failure of as-manufactured defective particles, was calculated, but no in-pile failure was observed. This suggests that the model for failure of particles with as-manufactured defects is conservative. However, more comparisons of calculations and in-pile data over a wider range of irradiation conditions are required before conclusions concerning the accuracy of HTGR design data and methods can be made.

An additional result of the postirradiation examination of FSV fuel element 1-0743 was verification of the techniques developed for performing nondestructive examinations of irradiated core components in the hot service facility at FSV using automated surveillance equipment. 
CONTENTS

ABSTRACT .............................

1. INTRODUCTION . . . . . . . . . . . . . . . 1-1

2. ELEMENT DESCRIPTION . . . . . . . . . . . . . 2-1

3. IRRADIATION CONDITIONS .................... 3-1

3.1. Irradiation History . . . . . . . . . . . 3-1

3.2. Power Distribution Measurements ........ 3-4

3.2.1. Description of Gamma Scanning System ..... 3-4

3.2.2. Radial Power Distributions ........ 3-5

3.2.3. Axial Power Distributions ....... . . 3-6

3.2.4. Fuel Rod Homogeneity . . . . . . . . 3-7

3.3. Fluence Measurements . . . . . . . . . 3-7

3.4. Temperature Measurements ... . . . . . . 3-8

3.5. Burnup Measurements . . . . . . . . . . . . 3-9

4. RESULTS OF POSTIRRADIATION EXAMINATION ........ . 4-1

4.1. Examination of Graphite Fuel Block ........ 4-1

4.1.1. Visual Examination.......... . 4-1

4.1.2. Metrological Examination ......... 4-1

4.2. Disassembly of Element . . . . . . . . . 4-4

4.2.1. Coring ............. 4 4-. 4

4.2.2. Plenum Depth Measurements ........ 4-5

4.2.3. Removal of Fuel Rods........... 4-5

4.3. Examination of Fuel Rods . . . . . . . . 4-6

4.3.1. Visual Examination .......... 4-6

4.3.2. Fue1 Rod Metrology .......... 4-7

4.3.3. Fuel Rod Strength Measurements . . . . . 4-8

4.3.4. Fission Gas Release ........... 4- . 4

4.3.5. Metallography ........... 4-10 
5. SUMMARY AND CONCLUSIONS . . . . . . . . . . . . . 5-1

5.1. Fuel Element Performance . . . . . . . . . . . 5-1

5.2. Verification of HTGR Core Design Methods . . . . . . 5-3

5.3. Verification of Nondestructive Examination Techniques • 5-7

6. ACKNOWLEDGMENTS . . . . . . . . . . . . . . . 6-1

7. REFERENCES . . . . . . . . . . . . . . . . . . 7-1

APPENDIX A: DISCUSSION OF BURNUP ANALYSIS . . . . . . . . . . A-1

FIGURES

2-1. FSV fuel element 1-0743 .. . . . . . . . . . 2-6

2-2. Temperature, fluence, and burnup monitor package . . . . 2-7

2-3. Top view of FSV fuel element 1-0743 showing locations of fuel holes containing precharacterized fuel rods . . . 2-8

2-4. Locations of fuel rods which underwent preirradiation fission gas release measurement and of monitor packages in FSV fuel element 1-0743 . . . . . . . . . . . . . . 2-9

2-5. Preirradiation fuel block measurements for FSV fuel element 1-0743 .................. . 2-11

2-6. Preirradiation fuel block dimensions for FSV fue1 element 1-0743 ................. 2-13

3-1. FSV reactor power history: SURVEY analysis of cycle 1 . 3-33

3-2. Local point numbering for GAUGE-SURVEY analysis . . . . 3-34

3-3. System for scanning FSV core components . . . . . . . 3-35

3-4. Scanning geometry 1: axial corner scans . . . . . . 3-36

3-5. Scanning geometry 2: axial sideface scans . . . . . 3-37

3-6. Scanning geometry 3: end-on scans . . . . . . . . 3-38

3-7. Measured time-averaged radial power distribution for FSV fuel element 1-0743 . . . . . . . . . . . . . 3-39

3-8. Measured EOL distribution for FSV fuel element 1-0743 . . . 3-40

3-9. Measured and calculated time-averaged axial power profiles for FSV fuel element 1-0743 . . . . . . . . . . . 3-41

3-10. Measured and calculated EOL axial power profiles for FSV fuel element 1-0743 ................

3-11. Cross-sectional view showing portion of element observed by detector for six axial scans averaged to give measured

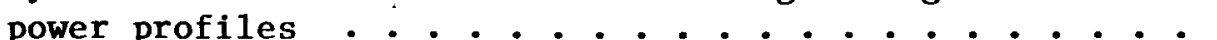


FIGURES (Continued)

3-12. Typical Cs-137 trace (partial) for axial scan of FSV fuel element 1-0743.............. . . . 3-44

3-13. Determination of irradiation temperatures for SiC pellets irradiated in FSV fuel element 1-0743 . . . . . . . . . . . 3-45

3-14. Core power history over the last $\sim 2 \times 10^{24} \mathrm{n} / \mathrm{m}^{2}$ (E > $29 \mathrm{fJ})_{\mathrm{HTGR}}$ exposure for FSV fuel element 1-0743 . . 3-46

4-1. FSV fuel element 1-0743, side face A . . . . . . . . 4-37

4-2. FSV fuel element 1-0743, side face B . . . . . . . . 4-38

4-3. FSV fuel element 1-0743, side face C . . . . . . . . 4-39

4-4. FSV fuel element 1-0743, side face D . . . . . . . . . 4-40

4-5. FSV fuel element 1-0743, side face E . . . . . . . . . 4-41

4-6. FSV fuel element 1-0743, side faces $E$ and $F$; numerous horizontal markings observed on face $F$. . . . . . . . 4-42

4-7. FSV fuel element 1-0743, top surface: fuel handling machine extension sleeve is at top of photograph . . . . 4-43

4-8. FSV fuel element 1-0743, close-up of fuel handling hole: small chip observed at edge of hole. . . . . . . . . 4-43

4-9. Coring tool. . . . . . . . . . . . . . . . . 4-44

4-10. Coring tool in operation .. . . . . . . . . . . . 4-45

4-11. Close-up of coring tool in operation . . . . . . . . . . . 4-45

4-12. Plenum depth measurement at top surface of fuel element . 4 4-46

4-13. Push-out drive in operation. . . . . . . . . . . . . . . . 4-47

4-14. Dual-tube receiving trough for fuel rod stacks . . . . . 4-47

4-15. Fuel rods irradiated in FSV fuel element 1-0743. Chipping and end cap cracking observed at ends of rods: (a) rod 12-2, end of rod is chipped; (b) rod 278-13: cracks in matrix end cap; rod 47-14: chipping at end of rod, failed fuel particles observed ... . . . . . . . . 4-48

4-16. Fuel rods irradiated in FSV fuel element 1-0743. Debonding observed on surfaces of rods: (a) rod 278-2; (b) rod 278-8; (c) rod 189-7; striation resulting from interaction of loose particles or graphite debris also observed . . . 4-49

4-17. Fuel rods irradiated in FSV fuel element 1-0743. Broken particles observed on end surfaces of fuel rods: (a) rod 12-2; (b) $\operatorname{rod} 12-7$................ 4- 450

4-18. Automated fuel rod dimensioning device used for metrology of fuel rods irradiated in FSV fuel element 1-0743 . . . . 4-51 
FIGURES (Continued)

4-19. Comparison of calculated and measured strain for fuel rods irradiated in FSV fuel element 1-0743 . . . . . . . . . 4 4-52

4-20. Load distribution during strength testing of a typical irradiated fuel rod from FSV fuel element 1-0743 . . . . 4-53

4-21. Photomicrographs representative of matrix phase . . . . . 4-54

4-22. Representative photomicrograph of composite of radial cross section of fuel rod irradiated in FSV fuel element $1-0743$ at $705^{\circ} \mathrm{C}$ to a fluence of $1.1 \times 10^{25} \mathrm{n} / \mathrm{m}^{2}$ (E $>29 \mathrm{fJ})_{\mathrm{HTGR}}$. The matrix phase is difficult to distinguish because the polished section was etched . . . 4-55

4-23. Photomicrographs of fissile and fertile particles . . . 4 4-56

4-24. Photomicrographs of examples of as-manufactured defective particles in irradiated fuel rods . . . . . . . 4-57

4-25. Photomicrographs of TRISO (Th, U) $\mathrm{C}_{2}$ particles apparently exhibiting slight fuel dispersion in buffer coating . . . 4-58

TABLES

1-1. Objectives of FSV surveillance program . . . . . . . . 1-3

2-1. Preirradiation fuel rod attributes for FSV fuel element $1-0743$. . . . . . . . . . . . . . . . 2-3

2-2. Fissile fuel particle attributes for FSV fuel element 1-0743 . . . . . . . . . . . . . . . . . . . 2-4

2-3. Fertile fuel particle attributes for FSV fuel element $1-0743$. . . . . . . . . . . . . . . . . 2-5

3-1. Envelope and time-averaged temperatures for FSV fuel element 1-0743 - element averge . . . . . . . . . 3-13

3-2. Envelope and time-averaged temperatures for FSV fuel element 1-0743 - survey local point 1 . . . . . . . 3-14

3-3. Envelope and time-averaged temperatures for FSV fuel element 1-0743 - survey local point 2. . . . . . . 3-15

3-4. Envelope and time-averaged temperatures for FSV fuel element 1-0743 - survey local point 3......... 3-16

3-5. Envelope and time-averaged temperatures for FSV fuel element 1-0743 - survey local point 4... . . . . . 3-17

3-6. Envelope and time-averaged temperatures for FSV fue1 element 1-0743 - survey local point 5........ . 3-18 
TABLES (Continued)

3-7. Envelope and time-averaged temperatures for FSV fuel element 1-0743 - survey local point 6... . . . . . 3-19

3-8. Envelope and time-averaged temperatures for FSV fuel element 1-0743 - survey local point 7 . . . . . . . 3-20

3-9. Fast neutron fluences for FSV fuel element 1-0743 . . . . 3-21

3-10. Fuel accountability for FSV fuel element 1-0743 . . . . . 3-22

3-11. Comparison of measured (Cs-137) and calculated time-averaged radial power distributions for FSV fuel element 1-0743 . . 3-23

3-12. Comparison of measured $(2 r-95)$ and calculated radial power distributions at EOL for FSV fue1 element 1-0743 . . . . 3-24

3-13. Dosimeter wire reactions . . . . . . . . . . . 3-25

3-14. Cross sections used for dosimetry calculations . . . . . 3-26

3-15. Comparison of calculated and measured neutron fluence for FSV fuel element 1-0743 . . . . . . . . . . . 3-27

3-16. Comparison of measured and calculated temperatures for SiC pellets irradiated in FSV fuel element 1-0743 . . . . . 3-28

3-17. Burnup measurements for FSV fuel element 1-0743 using destructive techniques . . . . . . . . . . . . 3-29

3-18. Burnup measurements for FSV fuel element 1-0743 using

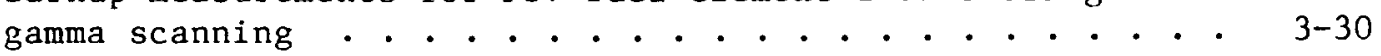

3-19. Comparison of calculated and measured fuel burnup for FSV fuel element 1-0743............. . . 3-31

3-20. Comparison of calculated and measured uranium isotopic concentrations for $\mathrm{UC}_{2}$ burnup monitors irradiated in FSV fuel element 1-0743................ 3- . . . 32

4-1. FSV fuel element 1-0743 axial dimensions . . . . . . . 4-13

4-2. FSV fue1 element 1-0743 transverse dimensional change location and minimum distance between coolant holes . . . . 4-14

4-3. FSV fuel element 1-0743 squareness datum planes . . . . . 4-15

4-4. FSV fuel element 1-0743 coolant hole diameters . . . . 4-16

4-5. FSV fuel element 1-0743 bow of six side faces . . . . . . 4-17

4-6. FSV fuel element 1-0743 displacement from vertical of six side faces .................. 4-18

4-7. FSV fuel element 1-0743 distance across flats . . . . . . 4-19

4-8. FSV fuel element 1-0743 length ........... . . 4-20 
TABLES (Continued)

4-9. FSV fuel element 1-0743 distances between centerlines

of coolant holes ................. . 4-21

4-10. FSV fuel element 1-0743 coolant hole diameters . . . . . 4-22

4-11. Accuracy of metrology robot measurements . . . . . . . 4-23

4-12. Calculated and measured irradiation-induced strains and bow for FSV fuel element 1-0743. . . . . . . . . . . 4-24

4-13. Plenum depth, fuel stack length, and push-out force measurements for FSV fuel element 1-0743 . . . . . . . 4-25

4-14. Broken fuel particles observed on surfaces of seventeen fuel rods from FSV fuel element 1-0743 . . . . . . . 4-26

4-15. Measured strains for fuel rods irradiated in FSV fuel element 1-0743 .. . . . . . . . . . . . . 4-27

4-16. Dimensional and strain data for fuel rods irradiated in fuel stack 12 of FSV fuel element 1-0743 . . . . . . . . 4-28

4-17. Dimensional and strain data for fuel rods irradiated in fuel stack 47 of FSV fuel element 1-0743 . . . . . . . . . 4-29

4-18. Dimensional and strain data for fuel rods irradiated in fuel stack 189 of FSV fuel element 1-0743 . . . . . . . . . 4-30

4-19. Dimensional and strain data for fuel rods irradiated in fuel stack 278 of FSV fuel element 1-0743 . . . . . . . . . 4-31

4-20. Dimensional and strain data for fuel rods irradiated in fue1 stack 285 of FSV fuel element 1-0743 . . . . . . . . 4-32

4-21. Compression testing of fuel rods from FSV fuel element $1-0743$. . . . . . . . . . . . . 4-33

4-22. Fission gas release measurements for fuel rods irradiated in FSV fuel element 1-0743 ............. 4-34

4-23. Fissile particle results of metallographic examination of fuel rods irradiated in FSV fuel element 1-0743 . . . . . 4-35

4-24. Fertile particle results of metallographic examination of fuel rods irradiated in FSV fuel element 1-0743 . . . . . 4-36 


\section{INTRODUCTION}

Fort St. Vrain (FSV) fuel element 1-0743 (serial number) was irradiated for 174 effective full-power days (EFPD) in core location 17.04.F.06; ${ }^{*}$ it experienced an average fast neutron exposure of about $0.95 \times 10^{25} \mathrm{n} / \mathrm{m}^{2}$

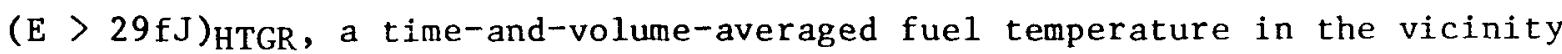
of $680^{\circ} \mathrm{C}$, fissile and fertile fuel particle burnups of approximately $6.2 \%$ and $0.3 \%$ fissions per initial heavy metal atom (FIMA), respectively, and a total burnup of $12,210 \mathrm{MWd} /$ tonne. The element was removed from the reactor during the first refueling in February 1979. After undergoing nondestructive examination in the hot service facility at FSV in July 1979, the element was shipped to General Atomic Company (GA) for extensive postirradiation examination (PIE).

The first part of the PIE involved visual and metrological examinations of the fuel block to verify the results obtained with the metrology robot system at FSV (Ref. 1). Next, extensive gamma scanning of the intact fuel element was performed to determine the distributions of measurable radioisotopes in the fuel. This exercise also served as a demonstration of the validity of gamma scanning as a method for determining fuel burnup and of the capabilities of the gamma scan robot. This device is currently being developed at GA for performing gamma spectroscopic examinations of FSV fuel elements at FSV.

Upon completion of the nondestructive portion of the PIE, the fuel hole plugs at the top of the element and the graphite containment at the bottom were cored and broken out, and the fuel rods were removed from the element. Examination of the fuel rods included visual examination, dimensional

\footnotetext{
${ }^{*}$ Core region 17 , column 4 , axial layer 6 (axial layer 3 of active core).
} 
characterization, fission gas release measurements, metallography, and compressive strength testing. Individual stacks of fuel rods were also gamma scanned to verify the results obtained from the earlier in situ scanning of the fuel. Four monitor packages containing SiC pellets, dosimetry wires, and $\mathrm{UC}_{2}$ particles for monitoring temperatures, neutron fluence, and fuel burnup were recovered from the element and subjected to analysis. The results of these analyses were compared with design code predictions.

The postirradiation examinations of FSV fuel element 1-0743 at FSV and at GA were performed as part of the surveillance program for the FSV hightemperature gas-cooled reactor (HTGR) sponsored by the Department of Energy (DOE). The FSV surveillance program includes nondestructive and destructive examinations of core components from the initial core reload segments. The purpose of these examinations is to verify the acceptable performance of the components and to acquire in-pile data over a wide range of irradiation conditions for verification of HTGR design data and methods. The benefit of these examinations will be early identification of performance defects and design margins. Specific objectives of the surveillance program are given in Table $1-1$. 
TABLE 1-1

OBJECTIVES OF FSV SURVEILLANCE PROGRAM

\begin{tabular}{|c|c|c|}
\hline Required Data & Objective & Postirradiation Examination Techniques \\
\hline $\begin{array}{l}\text { General mechanical integrity } \\
\text { and dimensional changes of } \\
\text { fuel rods at reactor tempera- } \\
\text { tures and fast neutron } \\
\text { exposures }\end{array}$ & $\begin{array}{l}\text { To judge irradiation limit for mechanical } \\
\text { integrity of fuel rods and fuel blocks, and } \\
\text { to permit the extrapolation necessary for } \\
\text { predicting fuel performance and confirming } \\
\text { existing design data based on irradiation } \\
\text { capsule experiments. }\end{array}$ & $\begin{array}{l}\text { Comparison of preirradiation and post- } \\
\text { irradiation dimensional measurements, } \\
\text { visual examination, comparison with pre- } \\
\text { irradiation photographs }\end{array}$ \\
\hline $\begin{array}{l}\text { Fuel block mechanical integ- } \\
\text { rity and critical dimensions, } \\
\text { Including bow at several } \\
\text { reactor temperatures and fast } \\
\text { neutron exposures }\end{array}$ & $\begin{array}{l}\text { To judge the irradiation limit for mechan- } \\
\text { lcal integrity of fuel rods and fuel } \\
\text { blocks, and to confirm design data and, in } \\
\text { confunction with fuel rod dimensional } \\
\text { change data, permit a confident prediction } \\
\text { of fuel performance }\end{array}$ & $\begin{array}{l}\text { Visual examination, comparison of pre- } \\
\text { irradiation and postirradiation dimensional } \\
\text { measurements }\end{array}$ \\
\hline $\begin{array}{l}\text { Fission product release rate } \\
\text { from fuel rods }\end{array}$ & $\begin{array}{l}\text { To evaluate the validity of design data and } \\
\text { confirm the limit for time-temperature- } \\
\text { irradiation with regard to fission product } \\
\text { release from the particles }\end{array}$ & $\begin{array}{l}\text { Burn-leach test for } S 1 C \text { integrity, com- } \\
\text { parison of preirradiation and post- } \\
\text { irradiation } \mathrm{Kr}-85 \mathrm{~m} \mathrm{R} / \mathrm{B} \text { values }\end{array}$ \\
\hline Fuel rod microstructure & $\begin{array}{l}\text { To Judge fuel performance relative to } \\
\text { kernel-coating interaction and coating } \\
\text { microstructure. These data are needed for } \\
\text { correlation with Irradiation capsule data } \\
\text { and out-of-pile data. }\end{array}$ & Metallographic examination \\
\hline $\begin{array}{l}\text { Mechantcal strength of fuel } \\
\text { rods }\end{array}$ & $\begin{array}{l}\text { To obtain knowledge of the change in } \\
\text { mechanical strength of fuel rods with } \\
\text { Increasing neutron exposure. The relative } \\
\text { integrity of the rod, and the exposure at } \\
\text { which integrity may be lost, could be } \\
\text { judged from this work. }\end{array}$ & $\begin{array}{l}\text { Uniaxial compression tests to fallure. } \\
\text { Includes irradiated fuel rods as well as } \\
\text { nonirradiated historical samples }\end{array}$ \\
\hline $\begin{array}{l}\text { Measured temperature, neutron } \\
\text { exposure, and fuel burnup }\end{array}$ & $\begin{array}{l}\text { To conflrm calculated temperatures, neutron } \\
\text { exposures, and fuel burnup }\end{array}$ & $\begin{array}{l}\text { Samples of SIC placed in fuel holes will } \\
\text { provide a temperature monitor. Standard } \\
\text { dosimetry wires developed for capsule } \\
\text { Irradiations placed in fuel holes will pro- } \\
\text { vide a measure of neutron exposure. UC } 2 \\
\text { particles placed in fuel holes will provide } \\
\text { a measure of the fissile burnup. Fertile } \\
\text { burnup can be determined through analysis } \\
\text { of ThC } 2 \text { particles from fuel rods. }\end{array}$ \\
\hline
\end{tabular}


$\bullet$ 


\section{ELEMENT DESCRIPTION}

Fuel element 1-0743 consisted of a standard H-327 graphite fuel body having 210 fuel holes, 6 burnable poison holes, and 108 coolant holes. The element (see Fig. 2-1) contained 3130 fuel rods consisting of $(\mathrm{Th}, \mathrm{U}) \mathrm{C}_{2}$ TRISO* fissile particles and $\mathrm{ThC}_{2}$ TRISo fertile particles bonded together by a carbonaceous matrix. The fuel rods were carbonized at $1800^{\circ} \mathrm{C}$ in a packed bed of $\mathrm{Al}_{2} \mathrm{O}_{3}$ powder. The nominal dimensions of the rods were $12.5 \mathrm{~mm}$ (0.49 in.) in diameter and $29.3 \mathrm{~mm}$ (1.94 in.) in length. Fuel rod and fuel particle attributes are given in Tables 2-1, 2-2, and 2-3. The element contained no lumped burnable poison.

Fuel element 1-0743 was one of 32 surveillance fuel elements irradiated in the initial core. Surveillance elements are readily distinguished from nonsurveillance elements by the fiducial holes drilled in each corner of the block. The dimensions of these elements were accurately characterized prior to loading the fuel. The elements contain fuel rods which were dimensionally characterized and measured for fission gas release prior to irradiation. In addition, SiC pellets, dosimetry wires, and $\mathrm{UC}_{2}$ fuel particles enclosed in 25.4-mm-1ong crucibles made of H-327 graphite are included in all surveillance elements to monitor temperature, neutron fluence, and fuel burnup. The design of the monitor packages is shown in Fig. 2-2.

*In the TRISO particle design, a layer of SiC is sandwiched between two layers of high-density pyrolytic carbon, which provides a composite pressure vessel to retain gaseous fission products. The SiC coating also provides a barrier against the diffusion of metallic fission products and increases the mechanical and dimensional stability of the particle during irradiation. An inner low-density, or buffer, coating adjacent to the fuel kernel provides a void volume to accommodate fission gases and kernel swelling and, in addition, attenuates fission product recoils. 
Fuel element 1-0743 contained 87 fuel rods that were dimensionally characterized prior to irradiation. These rods were loaded into fuel holes $12,47,157,189,278$, and 285. The locations of these holes are shown in Fig. 2-3. Preirradiation fission gas release measurements were made on a group of five rods, four of which were loaded into the fuel element. (The fifth rod was placed in permanent storage as a historical sample.) The four rods were situated in fuel stacks 47, 157, 278, and 285. The element included four monitor packages located in fuel stacks 12 and 278 . The axial locations of the fuel rods measured for fission gas release prior to irradiation and of the monitor packages are shown in Fig. 2-4. The preirradiation dimensional measurements for the fuel block are shown in Figs. 2-5 and $2-6$. 
TABLE 2-1

PREIRRADIATION FUEL ROD ATTRIBUTES FOR FSV FUEL ELEMENT 1-0743

Fue1 blend type:

CR-18-1-0165-1

Preirradiation fission gas release,

$\mathrm{Kr}-85 \mathrm{~m}$ at $1100^{\circ} \mathrm{C}$ :

$1.3 \times 10^{-4}$

Fraction exposed fuel after burning $\operatorname{rod}(a)$

$\mathrm{U}:$

$7.1 \times 10^{-3}$

Th:

$5.2 \times 10^{-3}$

Thorium contamination: (b)

$5.9 \times 10^{-5}$

Heavy metal loadings

$\mathrm{U}$ :

Th :

$0.148 \mathrm{~g} / \mathrm{rod}$

$4.082 \mathrm{~g} / \mathrm{rod}$

Impurities ( ppm)

$B$ :

2

Fe:

$\mathrm{S}:$

80

Ti :

V:

Residua1 hydrogen:

Residual ash:

$\mathrm{H}_{2} \mathrm{O}$ :

$\mathrm{Cl}$ :

280

40

40

100

2053

1

$--(\mathrm{c})$

Firing temperature:(d)

(a) Determined by burn leach test; value indicates hroken SiC 1ayer.

(b) Determined by hydrolysis test; value indicates exposed Th.

(c) (-) denotes no available data.

(d) Final heat treatment. 
TABLE 2-2

FISSILE FUEL PARTICLE ATTRIBUTES FOR FSV FUEL ELEMENT 1-0743

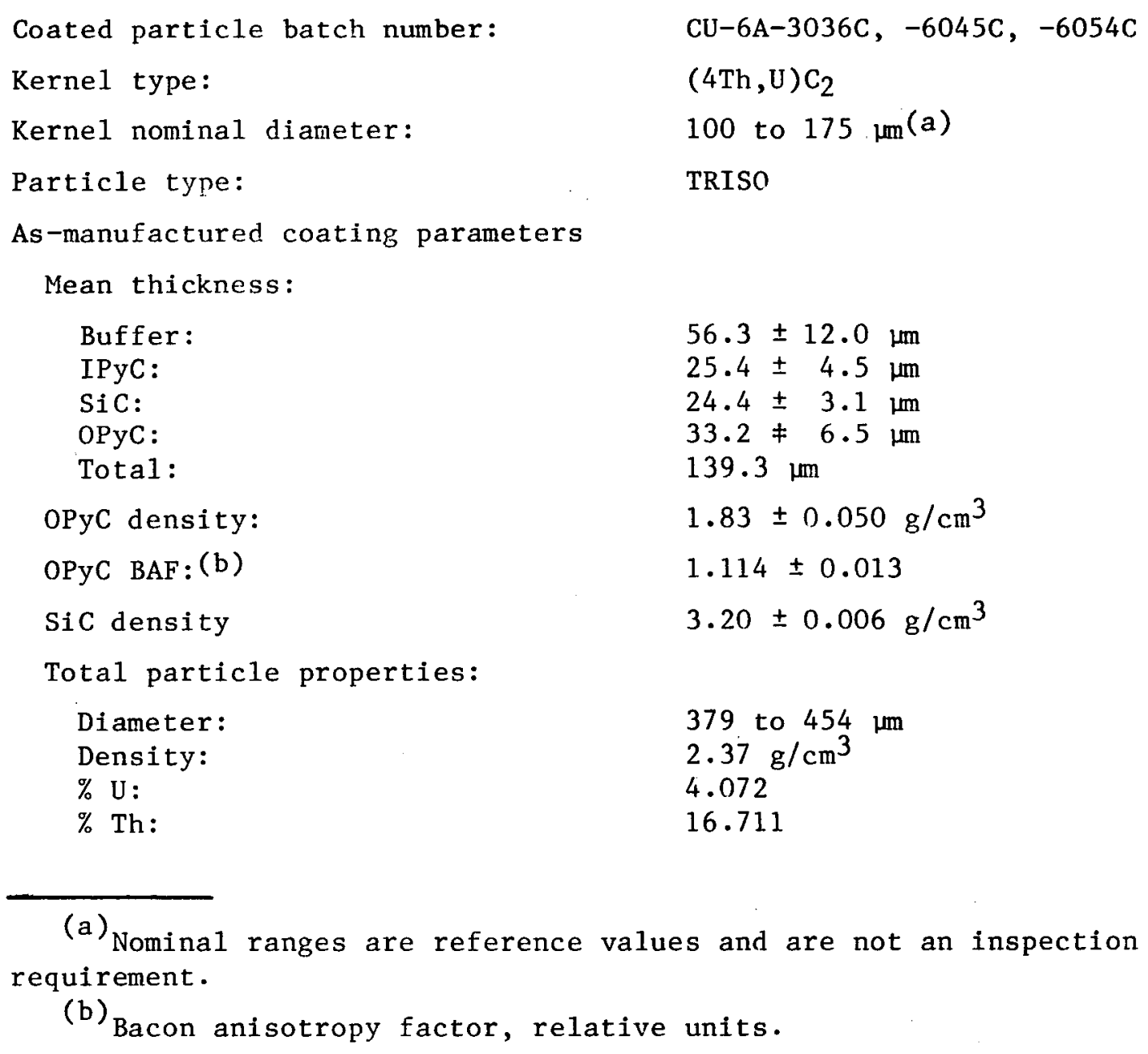

Total particle properties:

Diameter:

Density:

$\% \mathrm{U}$ :

$\%$ Th:

379 to $454 \mu \mathrm{m}$ $2.37 \mathrm{~g} / \mathrm{cm}^{3}$$$
4.072
$$

16.711

(a) Nominal ranges are reference values and are not an inspection requirement.

(b) Bacon anisotropy factor, relative units. 
TABLE 2-3

FERTILE FUEL PARTICLE ATTRIBUTES FOR FSV FUEL ELEMENT 1-0743

\begin{tabular}{|c|c|c|}
\hline & Fertile A & Fertile B \\
\hline Coated particle batch number & $\mathrm{CT}-6 \mathrm{~A}-1101 \mathrm{C}$ & $\mathrm{CT}-6 \mathrm{~B}-0127 \mathrm{C}$ \\
\hline Kernel type & $\mathrm{ThC}_{2}$ & $\mathrm{ThC}_{2}$ \\
\hline Kernel nominal diameter & 300 to $410 \mu \mathrm{m}(\mathrm{a})$ & 410 to $500 \mu \mathrm{m}(\mathrm{a})$ \\
\hline Particle type & TRISO & TRISO \\
\hline As-manufactured coating parameters & & \\
\hline Mean thickness & & \\
\hline Buffer & $52.5 \pm 13.1 \mathrm{um}$ & $56.7 \pm 14.9 \mathrm{\mu m}$ \\
\hline I PyC & $29.6 \pm 7.8 \mathrm{um}$ & $33.8 \pm 8.0 \mathrm{\mu m}$ \\
\hline $\mathrm{SiC}$ & $25.6 \pm 3.8 \mu \mathrm{m}$ & $26.4 \pm 4.5 \mu \mathrm{m}$ \\
\hline OPyC & $42.7 \pm 10.3 \mathrm{\mu m}$ & $44.0 \pm 8.3 \mu \mathrm{m}$ \\
\hline Total & $150.4 \mathrm{um}$ & $160.9 \mathrm{um}$ \\
\hline OPyC density & $1.773 \pm 0.086 \mathrm{~g} / \mathrm{cm}^{3}$ & $1.799 \pm 0.037 \mathrm{~g} / \mathrm{cm}^{3}$ \\
\hline OPyC BAF(b) & $1.14 \pm 0.035$ & $1.16 \pm 0.039$ \\
\hline SiC density & $3.19 \pm 0.016 \mathrm{~g} / \mathrm{cm}^{3}$ & $3.19 \pm 0.016 \mathrm{~g} / \mathrm{cm}^{3}$ \\
\hline Total particle properties & & \\
\hline Diameter & 601 to $711 \mathrm{~m}$ & 732 to $822 \mathrm{~lm}$ \\
\hline Density & $3.17 \mathrm{~g} / \mathrm{cm}^{3}$ & $3.45 \mathrm{~g} / \mathrm{cm}^{3}$ \\
\hline$\% \mathrm{U}$ & & 0 \\
\hline$\% \mathrm{Th}$ & 45.32 & 51.97 \\
\hline
\end{tabular}

(a) Nominal ranges are reference values and are not an inspection requirement .

(b) Bacon anisotropy factor, relative units. 
ALL DIMENSIONS IN mm
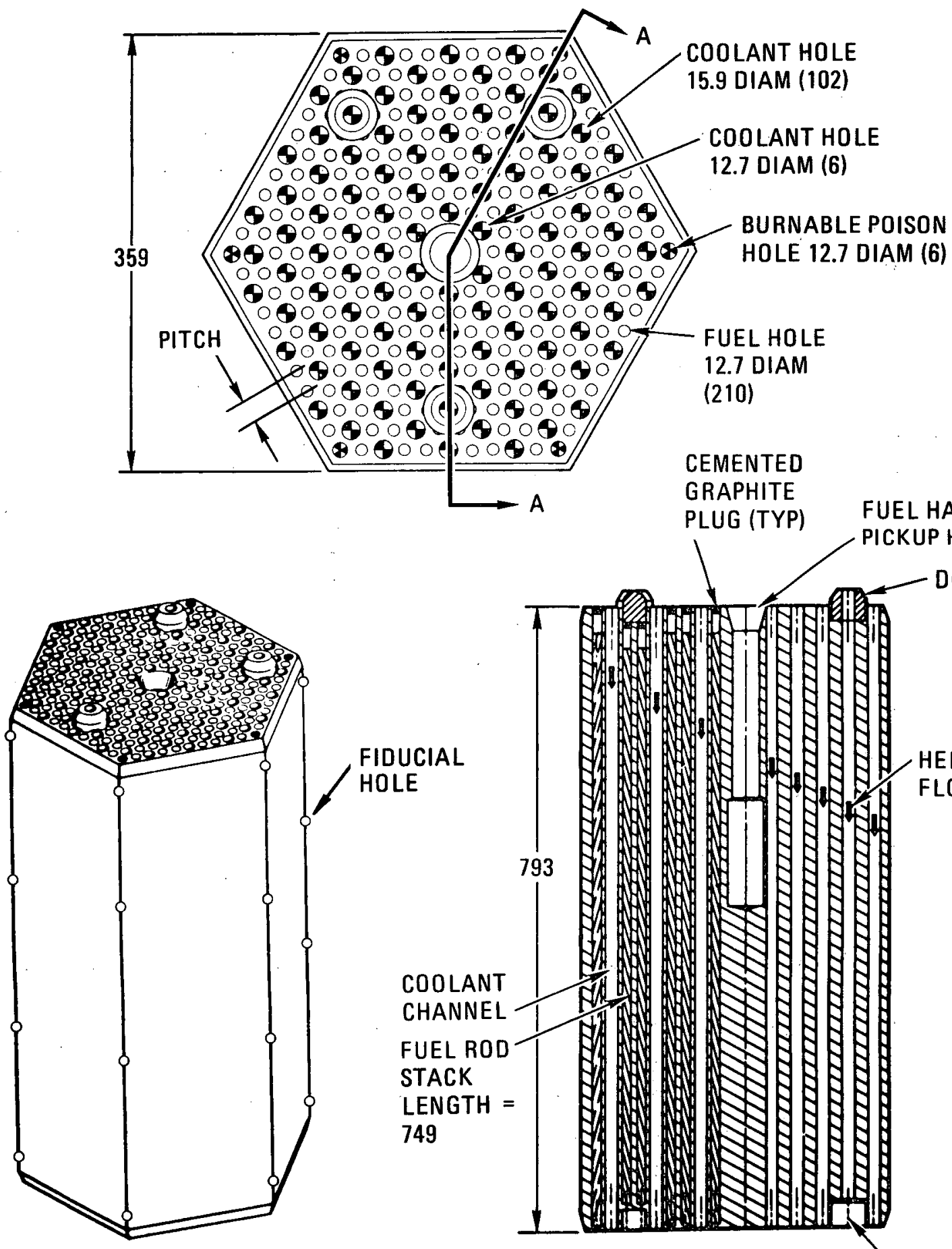

FIDUCIAL HOLE

A

\section{GRAPHITE}

PLUG (TYP) FUEL HANDLING PICKUP HOLE DOWEL PIN
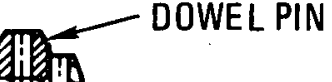

Fig. 2-1. FSV fuel element 1-0743 


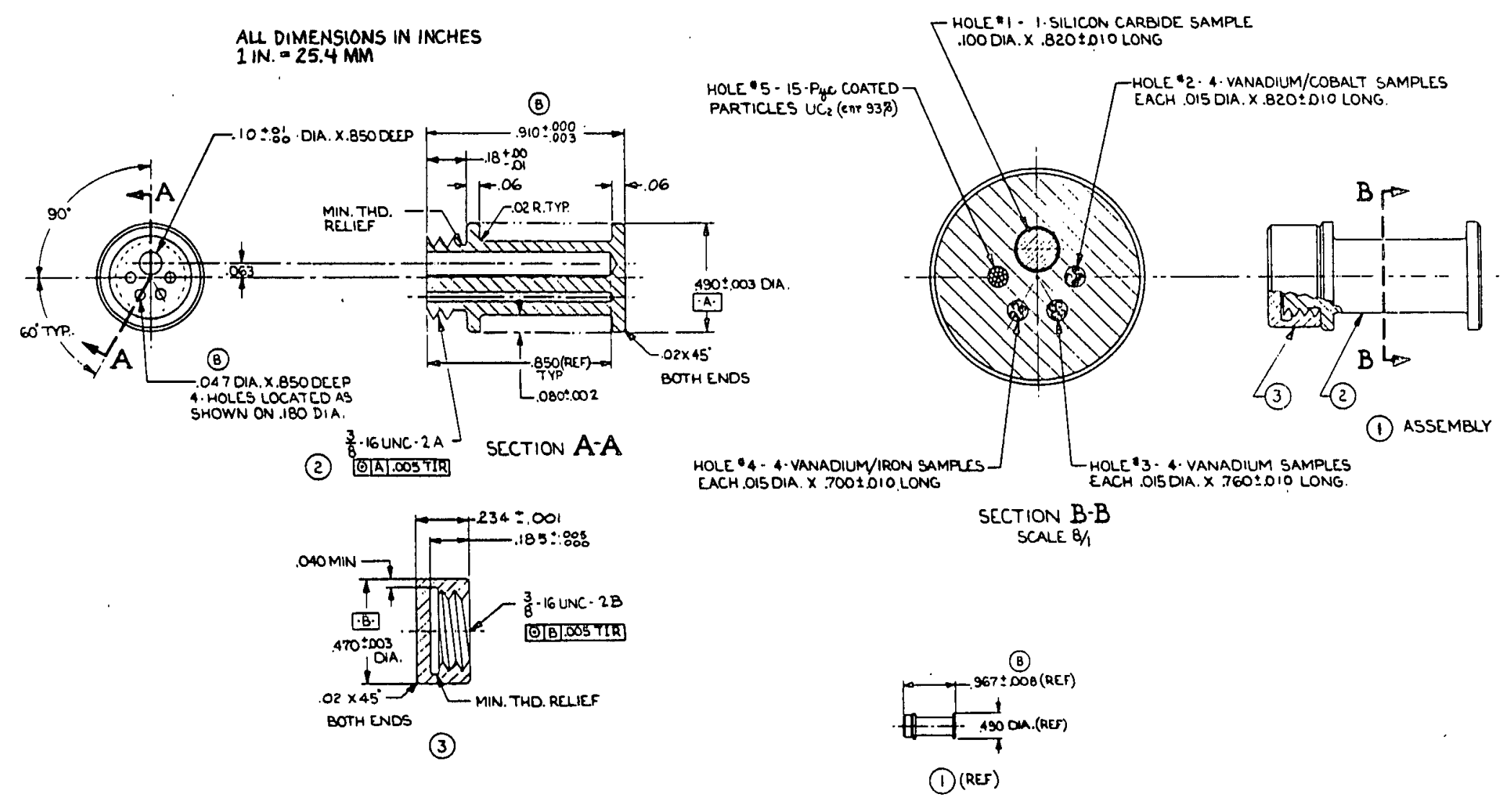

Fig. 2-2. Temperature, fluence, and burnup monitor package 


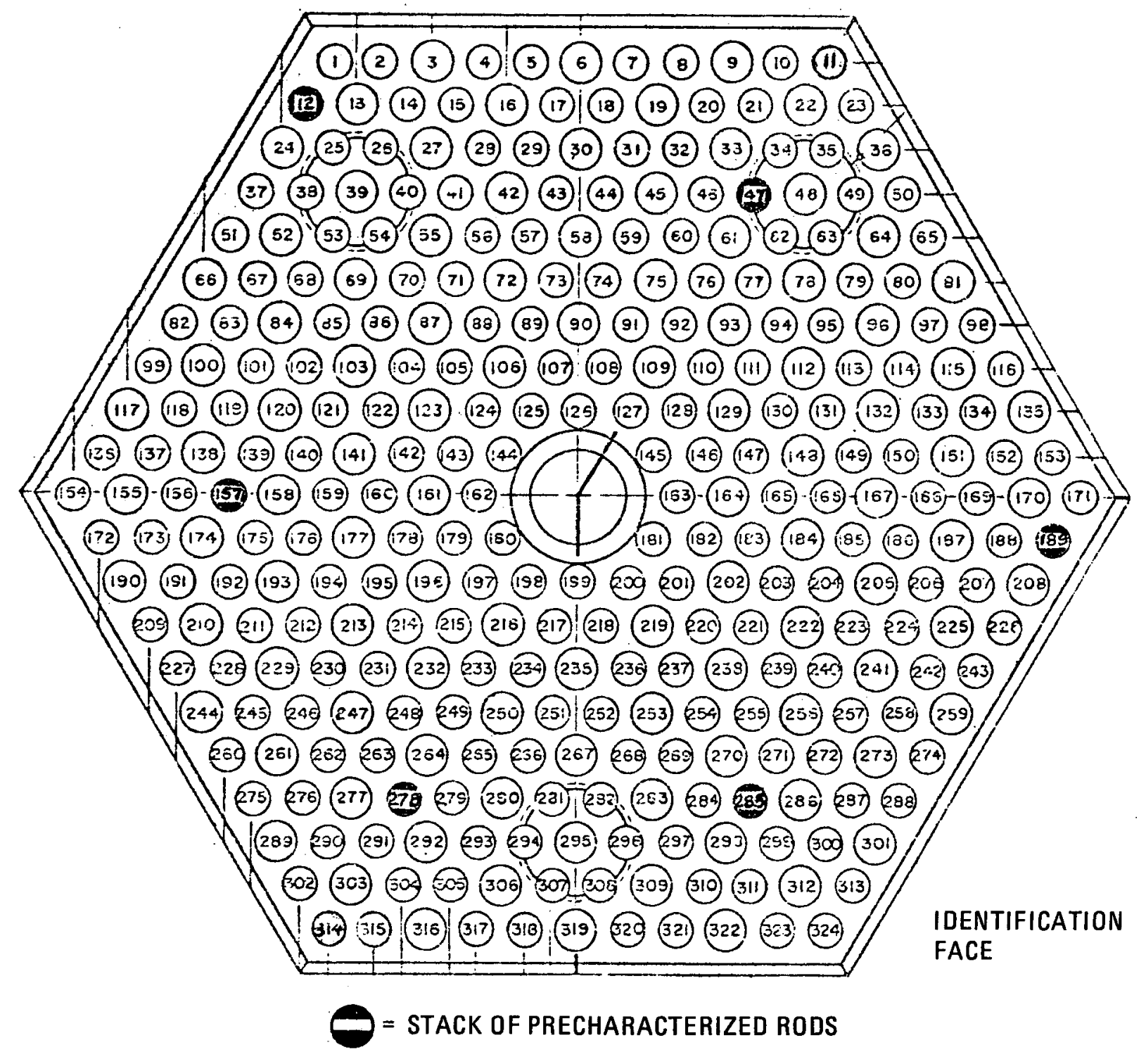

Fig. 2-3. Top view of FSV fuel element 1-0743 showing locations of fue1 holes containing precharacterized fuel rods 


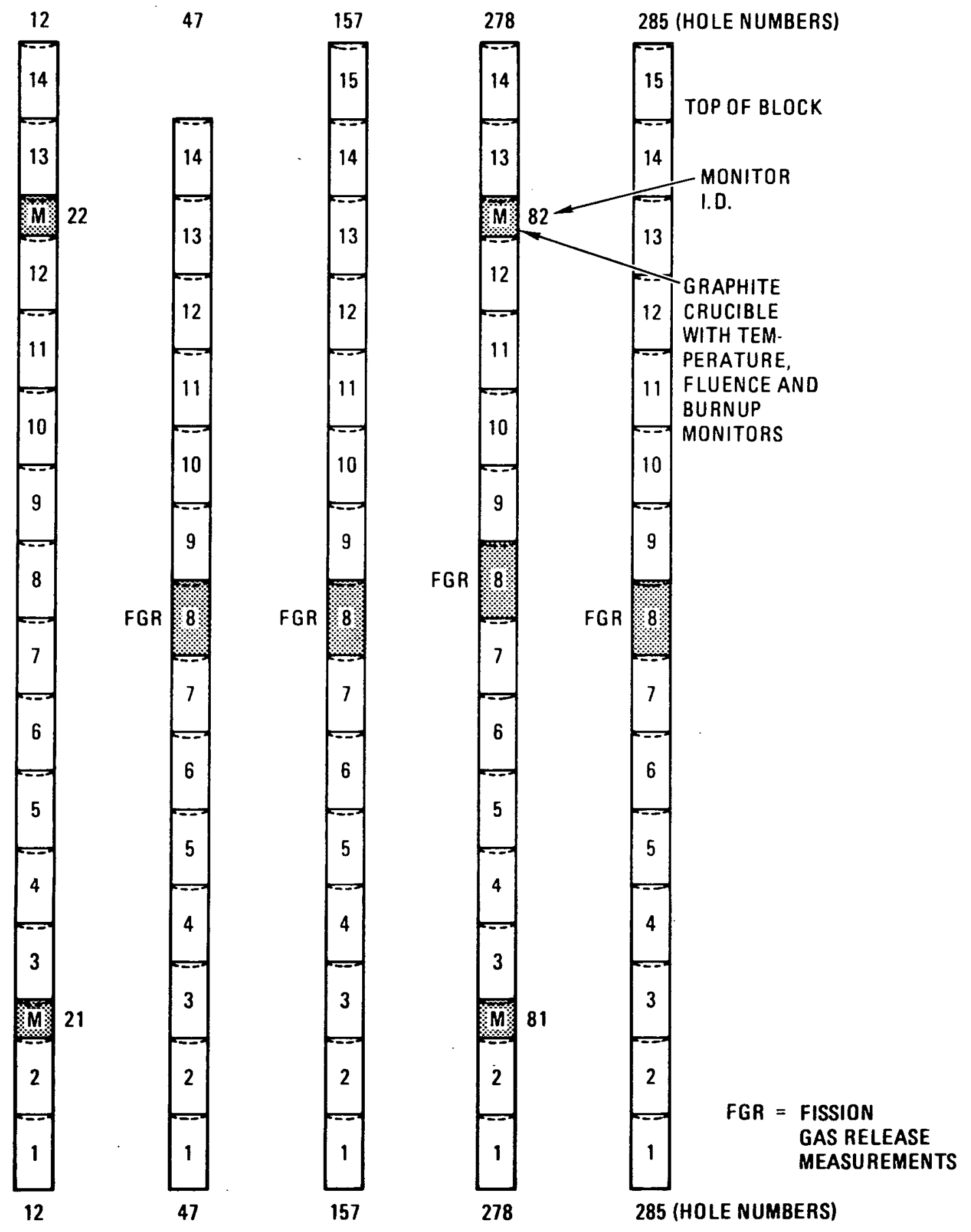

Fig. 2-4. Locations of fuel rods which underwent preirradiation fission gas release measurement and of monitor packages in FSV fuel element 1-0743 
• 

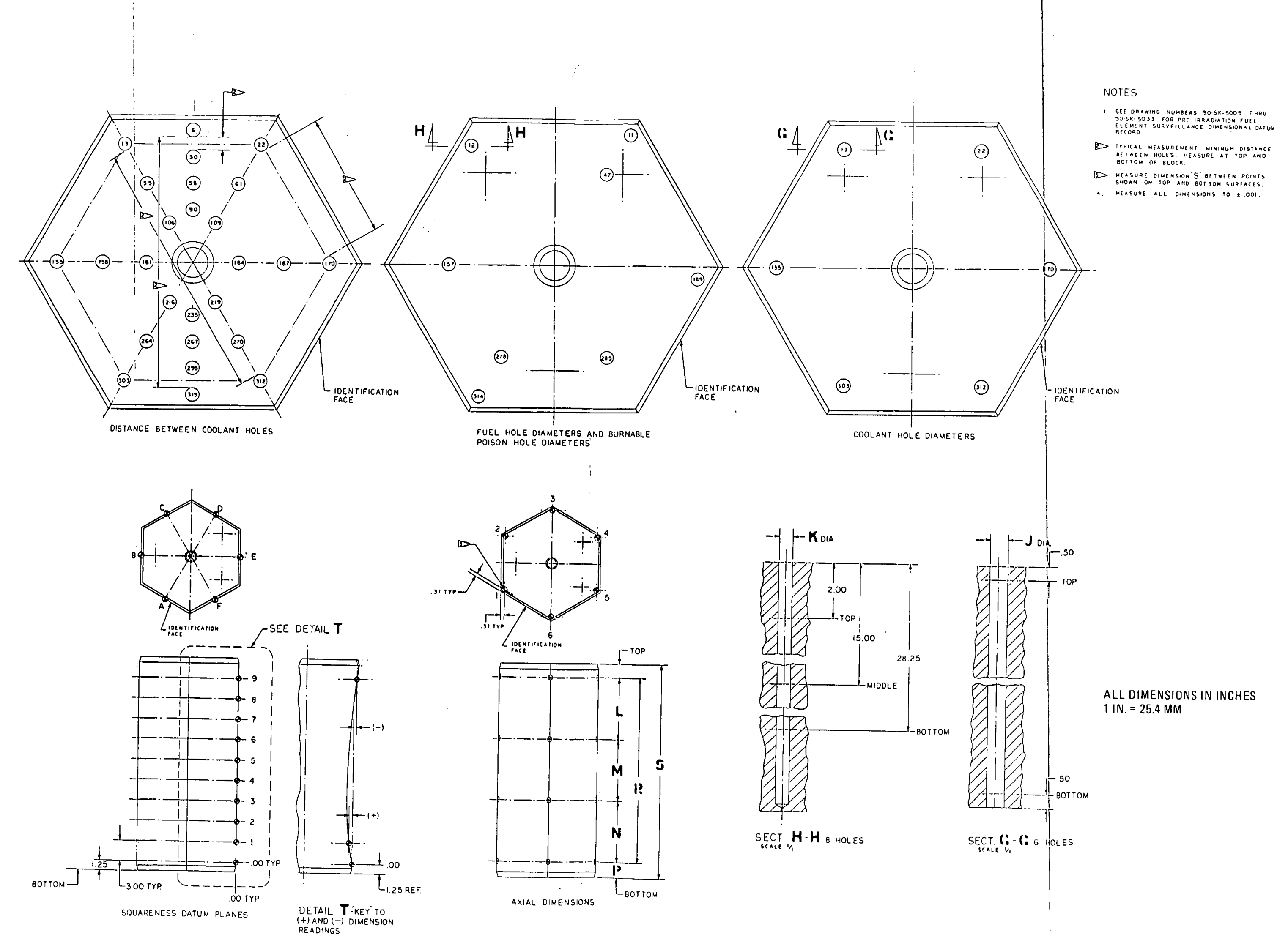

A $=254$ IONS IN INCHES 1N. 25.4 m

Fig. 2-5. Preirradiation fuel block measurements for FSV fue1 element 1-0743 


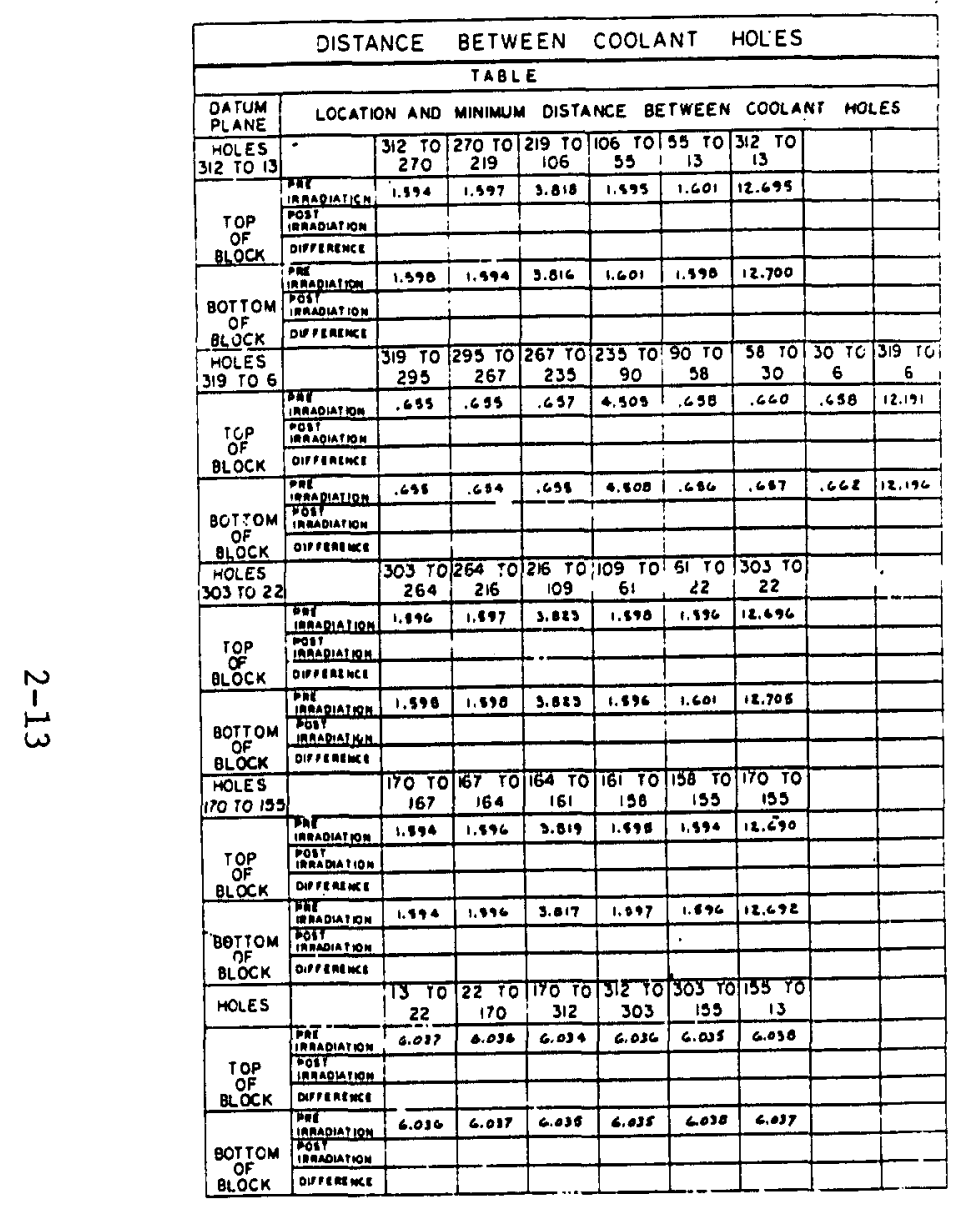

ALL OIMENSIONS IN IMCHEE, I IN. • 25.4 MM
FUEL HOLE DIAMET ERS AND
BURNABLE POISON HOLE DIAMETERS

\begin{tabular}{|c|c|c|c|c|}
\hline \multicolumn{5}{|c|}{ TABLE } \\
\hline \multirow{3}{*}{$\begin{array}{l}\text { HOLE } \\
\text { NO. }\end{array}$} & & \multicolumn{3}{|c|}{ HOLE DIAMETER } \\
\hline & & TOP & MIDOLE & BOCTOM \\
\hline & & $K_{\text {DIA }}$ & $K_{\text {DIA. }}$ & $K_{\text {OIA. }}$ \\
\hline \multirow{3}{*}{11} & 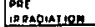 & 198 & .4979 & \\
\hline & 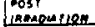 & & & \\
\hline & Dorrentexer & & & \\
\hline \multirow{3}{*}{12} & 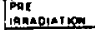 & 498 & .490 & (5985 \\
\hline & $\begin{array}{l}\text { Post } \\
\text { inenourion }\end{array}$ & & & \\
\hline & torrinter & & & \\
\hline \multirow[b]{2}{*}{47} & 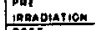 & .40 & .098 & .4975 \\
\hline & 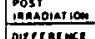 & & & \\
\hline \multirow{3}{*}{137} & apat & 4919 & .47 &. \\
\hline & & & & \\
\hline & Orictenest & & $\therefore$ & \\
\hline \multirow{3}{*}{189} & infopiation & 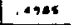 & ...ess & .40 \\
\hline & & & & \\
\hline & 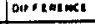 & & & \\
\hline \multirow[b]{2}{*}{278} & Paration & .40 & .90 & .9913 \\
\hline & Labiantion & & & \\
\hline \multirow{3}{*}{203} & 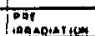 & 498 & . & .073 \\
\hline & 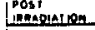 & & & \\
\hline & | & & & 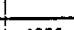 \\
\hline \multirow{2}{*}{314} & 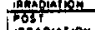 & 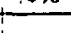 & 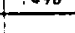 & \\
\hline & Darreten oxt & & & \\
\hline
\end{tabular}

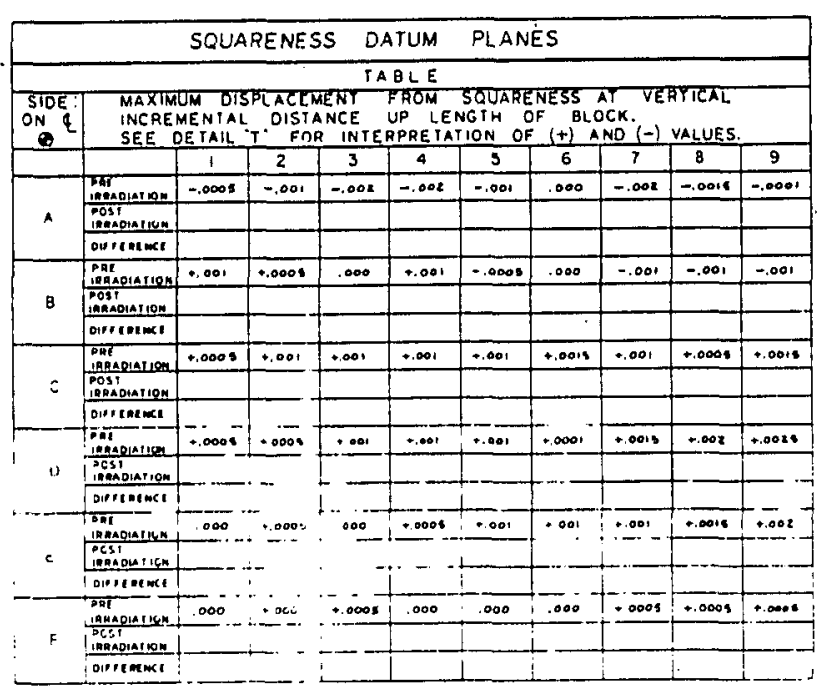

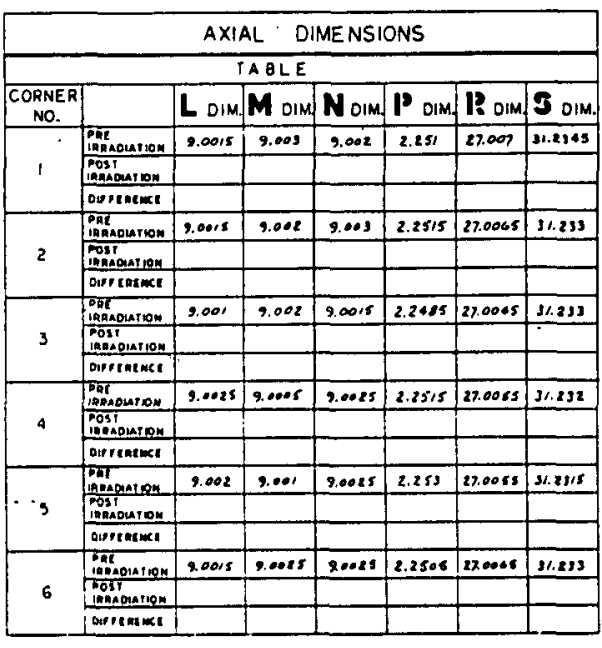

COOLANT HOLE DIAMETERS

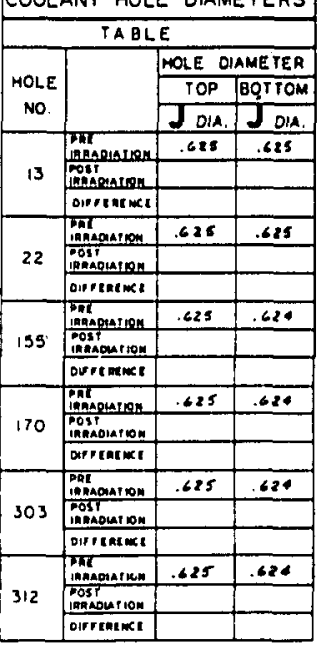

Fig. 2-6. Preirradiation fuel block dimensions for FSV fuel element 1-0743 
。
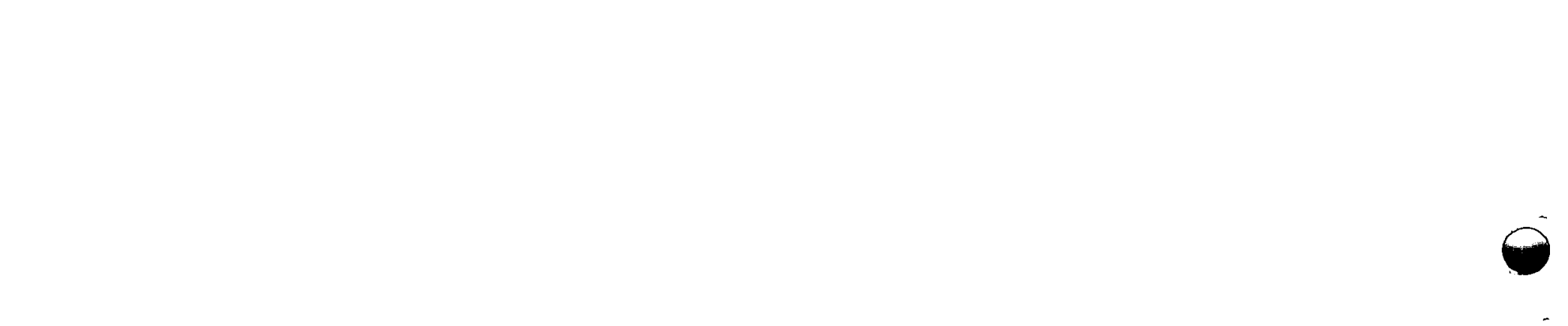

-

, 


\section{IRRADIATION CONDITIONS}

\subsection{IRRADIATION HISTORY}

Fort St. Vrain fuel element 1-0743 was irradiated in core location 17.04.F.06 from July 3, 1976 until February 1, 1979. During this time, the cumulative core power was $146,500 \mathrm{MWd}$. The reactor was at significant power ( $>10 \mathrm{MW}$ ) for aproximately 500 days, and the average reactor power was about 293 MW (35\% power). In terms of EFPD, ${ }^{*}$ the irradiation time was 174 days .

The irradiation history of the element has been simulated using the following HTGR design codes:

GAUGE (Ref. 2): a two-dimensional, four-group neutron diffusion and core depletion code. GAUGE treats the core as a single layer and calculates nuclide densities as a function of time and radial core location.

GATT (Ref. 3): a three-dimensiona1, four-group neutron diffusion and core depletion code. GATT calculates nuclide densities as a function of time and axial and radial core location.

FEVER (Ref. 4): a one-dimensional, multigroup neutron diffusion and depletion program for calculating nuclide densities as a function of axial core location.

\footnotetext{
(842 MW).

*An EFPD is the equivalent of 1 day of operation at full power
} 
BUG-2 (Ref. 5): a two-dimensional, multigroup neutron diffusion and depletion program for calculating nuclide densities as a function of axial core location for fuel assemblies influenced by partially inserted control rods.

SURVEY (Ref. 6): a computer program for the thermal and fuel performance analysis of HTGR fuel elements. The code is used to perform coarse mesh survey analyses for large numbers of spatial positions, calculating a time history of the irradiation conditions and fuel performance for each space point. SURVEY calculations are based on radial power distributions obtained from GAUGE and axial power distributions obtained from FEVER and BUG-2.

SURVEY/STRESS (Ref. 7): a computer program for calculating stresses, strains, and deformations in a large HTGR fuel block using viscoelastic beam theory. The code employs a relatively simple model and is used to survey an entire core to identify elements with high stresses. Once identified, these elements are subjected to more rigorous analyses using codes which employ more complex models. The irradiation conditions used in the stress calculations are obtained from SURVEY.

The reactor operating power is logged on an hourly basis. However, because of the numerous changes in power during cycle 1 , an analysis of the actual power history would be prohibitively expensive. Consequent1y, the power history for cycle 1 was reduced to 335 time intervals of approximately uniform power. Cycle 1 operation was simulated with the GAUGE code using this "detailed" power history. A SURVEY analysis of selected elements, including fuel element 1-0743, was then performed based on the GAUGE results. The number of time intervals was further reduced from 335 to 36 representative time intervals for this analysis. The power history for the SURVEY analysis is shown in Fig. 3-1. Finally, a SURVEY/STRESS analysis was performed based on the SURVEY results. In GAUGE, SURVEY, and SURVEY/ STRESS analyses, calculations are performed at seven radial locations per element, as shown in Fig. 3-2. 
In addition to the detailed GAUGE analysis, a three-dimensional burnup analysis of cycle 1 was performed using GATT. The primary objective was to obtain the fuel accountability for the segment 1 fuel elements. Power distributions, neutron fluences, and fuel burnup were also obsained. Because of the great expense of running GATT, the power history had to be reduced to a relatively few time intervals. For the GATT analysis, described in Ref. 8 , cycle 1 was represented by 11 time intervals.

A second GAUGE analysis of cycle 1, based on the 11-time-interval power history, and a FEVER code analysis, specifically for fuel element 1-0743, were also performed (Ref. 9). SURVEY code analyses based on the results of these analyses and the results from GATT were not performed.

Envelope and time-averaged temperatures calculated for fuel element 1-0743 are given in Tables 3-1 through 3-8. Fast neutron fluences are shown in Table 3-9. The time- and volume-averaged graphite and fuel temperatures for the element were $646^{\circ} \mathrm{C}$ and $680^{\circ} \mathrm{C}$, respectively. The maximum fuel temperature experienced by the element was $935^{\circ} \mathrm{C}$. The element average fast neutron fluence was $0.95 \times 10^{25 \mathrm{n} / \mathrm{m}^{2}}$ ( $\mathrm{E}>29 \mathrm{fJ}$ ) HTGR, and the maximum fast $\mathrm{flu}-$ ence was $1.1 \times 10^{25} \mathrm{n} / \mathrm{m}^{2}$ ( $E>29 \mathrm{fJ}$ ) HTGR. Temperatures and fluences were lowest on the side of the element adjacent to the central column of region 17 and highest on the opposite side. The differences between the highest and lowest time-averaged graphite and fuel temperatures in the element are $68^{\circ}$ and $70^{\circ} \mathrm{C}$, respectively. The difference between the highest and lowest fast fluence is $0.28 \times 10^{25} \mathrm{n} / \mathrm{m}^{2}$ (E $>29 \mathrm{fJ}$ ) HTGR. The fissile and fertile burnups remained approximately constant over the length of the element and were $6.2 \%$ and $0.3 \%$ FIMA. Fuel burnups were not computed as a function of radial location.

The above results were obtained from the SURVEY-detailed GAUGE analysis. The fuel accountability for element 1-0743 (obtained from GATT) is given in Table 3-10. 


\subsection{POWER DISTRIBUTION MEASUREMENTS}

As part of the PIE of FSV surveillance element 01-0743, extensive gamma scanning was performed to determine the relative distributions of measurable radioisotopes in the fuel. These data provide information on the power distribution in the element during irradiation and can be used to verify nuclear design calculations and to better define the nuclear and thermal parameters corresponding to observed materials performance.

Of particular value are the measured $\mathrm{Cs}-137$ and $\mathrm{Zr}-95$ distributions. Since Cs-137 is a direct-yield isotope from the fission of U-235 and U-233 and has a half-life (30 yr) far greater than the irradiation period for the element, the Cs-137 distribution is representative of the time-averaged power distribution, providing that significant quantities of Cs-137 did not escape from the fuel. This can reasonably be assumed to be the case, since the element contained all-TRISO fuel and experienced relatively low temperature $\left(<1000^{\circ} \mathrm{C}\right)$ and neutron exposure $\left[\sim 1.0 \times 10^{25} \mathrm{n} / \mathrm{m}^{2}(\mathrm{E}>29 \mathrm{fJ})_{\mathrm{HTGR}}\right]$. $\mathrm{Zr}-95$ is also a direct-yield isotope from the fission of U-235 and U-233 but has a half-life of only 65.5 days. The $\mathrm{Zr}-95$ distribution is therefore representative of the power distribution at end of life (EOL).

A brief discussion of how the gamma scanning was performed is presented below. The measured $\mathrm{Cs}-137$ and $\mathrm{Zr}-95$ distributions are then presented and compared with predicted power distributions. Homogeneity data obtained for segment 1 fuel rods are also discussed.

\subsubsection{Description of Gamma Scanning System}

The gamma scanning system consists of a robotic device that accurately positions the fuel element in front of a collimator aligned with an outof-cell high-resolution $\mathrm{Ge}(\mathrm{Li})$ detector. The signal from the detector is sent to a Nuclear Data (ND) 6620 data acquisition system and to a singlechannel analyzer (SCA)-ratemeter-recorder system. The ND 6620 system collects the spectra and stores them on a disk, where they are later 
accessed and analyzed by various spectral analysis programs. The SCAratemeter-recorder system monitors and traces the Cs-137 distribution. A collimator constructed of aluminum and having a length of $1759 \mathrm{~mm}$ and a $15.9 \times 12.7$ mm cross-sectional opening is used for all gamma scanning. An overview of the system is shown in Fig. 3-3.

The in situ gamma spectroscopic examination of FSV surveillance element 01-0743 was performed using three basic scanning geometries. These geometries, which are referred to as the axial corner, axial side-face, and endon scanning geometries, are shown in Figs. 3-4, 3-5, and 3-6, respectively.

Axial scanning was performed as the fuel block was moved slowly past the collimator. Spectra were acquired at intervals approximately equal to the length of a fuel rod. The acquisition times for an axial corner scan and for a side-face scan (one rod length per scan) were approximately 8 and 5 min, respectively. The length of the block was scanned a total of 15 times, 9 times via the side-face scaning geometry and 6 times via the corner scanning geometry. Each end-on scan was obtained by summing a series of static scans that traversed the cross section of the fuel stack under observation. The acquisition time for an end-on scan was approximately 6 min. End-on scans of 70 fuel stacks were acquired. The end-on scans were performed with the bottom of the block facing the detector. All in situ gamma scanning was performed in an automated mode under the direction of the ND 6620 computer.

\subsubsection{Radial Power Distributions}

The normalized radial distributions of $\mathrm{Cs}-137$ and $\mathrm{Zr}-95$ in FSV surveillance element 01-0743 are shown in Figs. 3-7 and 3-8. The Cs-137 distribution is compared with calculated time-averaged power distributions in Table 3-11 and the $\mathrm{Zr}-95$ distribution with calculated radial power distributions at EOL in Table 3-12. Little intrablock tilting in the radial power distribution was calculated and little was observed. For timeaveraged power the maximum observed tilt (difference between the 
highest and lowest relative power factor) was $9 \%$, and the maximum calculated tilts were $13 \%$ for the SURVEY-detailed GAUGE analysis and $4 \%$ for the $11-$ time-interval GAUGE analysis. The reason for the relatively large difference in the calculated tilts has not been determined. At EOL, the maximum observed tilt was $8 \%$ and the calculated tilts were $4 \%$ for the SURVEYdetailed GAUGE analysis, $3 \%$ for the GATT analysis, and $4 \%$ for the 11 -timeinterval GAUGE analysis. The agreement between calculated and measured local-to-block average power factors was within $7.5 \%$ for all local points. This is wel1 within the $\pm 10 \%$ uncertainty $(1 \sigma)$ generally quoted for GAUGE calculations and confirmed in Ref. 10 .

\subsubsection{Axial Power Distributions}

Measured and calculated axial power distributions for fuel element 1-0743 are shown in Figs. 3-9 (time averaged) and 3-10 (EOL). The measured profiles are normalized $\mathrm{Cs}-137$ and $\mathrm{Zr}-95$ profiles obtained by averaging the results of six axial side-face scans. A cross-sectional view of the portion of the element observed by these scans is shown in Fig. 3-11. The calculated profiles were otained with the FEVER code.

The agreement between the measured and calculated profiles at EOL is excellent. The time-averaged profiles are also in good agreement except near the bottom of the element, where the disagreement approaches $10 \%$. The reason for the discrepancy near the bottom of the element is that the FEVER model cannot account for the control rod in region 34, which was partially inserted during much of cycle 1. The effect of this partially inserted control rod was to tilt the axial power distribution toward the bottom of the element. At EOL the rod was nearly withdrawn, so its influence on the axial power distribution was minimal. This explains the improved agreement between the measured and calculated power profiles at EOL. 


\subsubsection{Fuel Rod Homogeneity}

The distribution of $\mathrm{Cs}-137$ and other measured radioisotopes along the length of individual fuel rods was observed to be markedly U-shaped, with the activity near the ends being almost twice the activity in the middle for many of the rods. A portion of a typical Cs-137 trace for an axial scan is shown in Fig. 3-12. Nearly all rods were observed to have this U-shaped profile, suggesting a manufacturing process that tended to segregate the fissile particles toward the ends of the rods. This has been confirmed via gamma scanning of unirradiated fuel (Ref. 11), which showed the U-235 distribution in segment 1 fuel rods to have the same shape as the Cs-137 distribution.

\subsection{FLUENCE MEASUREMENTS}

Three types of dosimeters were included in the monitor packages irradiated in fuel element 1-0743: V-Co and pure $V$ wires for measuring the thermal neutron fluence and $V-F e$ wires for measuring the fast neutron fluence. The reactions of interest for the dosimeters are listed in Table 313. All dosimeters were recovered from the four monitor packages and submitted for gamma ray analysis. The measured activities for the radionuclides of interest were back-decayed to EOL and used to compute the fast and thermal fluences for each monitor location. The cross sections used in the calculations were obtained from Ref. 12 and are listed in Table 3-14.

Measured fluences are compared with predictions in Table 3-15. The predicted fluences were obtained from the SURVEY-detailed GAUGE, GATT, and 11-time-interval GAUGE analyses of cycle 1 . The agreement between measured and calculated fast fluences is excellent (within $6 \%$ for all comparisons). The agreement between measured and calculated thermal fluences is not as good. The predicted thermal fluence is $11.9 \%$ smaller than the thermal fluence determined from the V-Co dosimeters and $39.9 \%$ greater than the fluence 
determined from the pure $V$ dosimeters. The fluence established from the $V$ dosimeters is believed to be in error, but it is not certain at this time whether the error is due to using the wrong cross section for the ${ }^{51} \mathrm{~V}(n, \gamma)^{52} \mathrm{~V}$ reaction or to a defect in the technique for measuring the ${ }^{52} \mathrm{Cr}$ resulting from the $\beta$ decay of ${ }^{52} \mathrm{~V}$.

\subsection{TEMPERATURE MEASUREMENTS}

Irradiation of SiC produces a small increase in macrodimensions which is related to the irradiation temperature. Postirradiation annealing at progressively higher temperatures causes no change to occur in the SiC until a critical temperature is reached, after which the length decreases as the irradiation damaged is annealed out. This decrease in length is approximately linear with increasing temperature. The critical temperature, which is determined from the intersection of the regression lines for the two essentially linear portions of the annealing curve, is related to the irradiation temperature.

Irradiation temperatures for the four SiC pellets recovered from the monitor packages were determined via isochronal annealing. The pellets were annealed for a period of about $1 \mathrm{hr}$ at temperatures from $200^{\circ}$ to $1100^{\circ} \mathrm{C}$ in $50^{\circ} \mathrm{C}$ increments. The annealing curves for the SiC pellets are shown in Fig. 3-13. Irradiation temperatures were determined from the annealing curve intersection temperatures using the calibration curve for SiC temperature monitors presented in Ref. 13 .

A comparison of measured and calculated temperatures for the monitors is made in Table 3-16. The measured temperatures are assumed to be approximately representative of temperatures during periods of higher reactor power operation shortly before shutdown. This is thought to be the case since irradiation damage accumulated at low temperatures would have been annealed out at the relatively high temperatures experienced by the samples during these periods, and since the period of lower power (and temperature) operation just prior to shutdown was too short for a significant accumulation of 
low-temperature-related irradiation damage. The core power over the last $\sim 2 \times 10^{20} \mathrm{n} / \mathrm{cm}^{2}(\mathrm{E}>29 \mathrm{fJ})_{\mathrm{HTGR}}$ is shown in Fig. 3-14.* Calculated temperatures were obtained from SURVEY-calculated peak fuel and coolant temperatures at the axial locations of neighboring fuel rods using a factor obtained with the TAC-2D (Ref. 14) code.**

The calculated temperature for each temperature monitor was approximately $25^{\circ} \mathrm{C}$ greater than the measured temperature. In all cases, the calculated temperature was within the $95 \%$ confidence limits for the measured temperature.

\subsection{BURNUP MEASUREMENTS}

$\mathrm{UC}_{2}$ fissile particles from three of the four monitor packages and $\mathrm{ThC}_{2}$ fertile particles obtained from neighboring fuel rods were submitted for burnup analysis. The fissile particles were analyzed using (1) a radiochemistry method employing $\mathrm{Cs}^{-137}$ as a burnup monitor and (2) a mass spectrometric method in which burnup was determined from changes in uranium isotopic composition. The fertile particles were analyzed using a method in which the thorium content in the particles was deduced from the Pa-233 activity following a short irradiation in the TRIGA test reactor. The details of the analyses are provided in Appendix A. The results of the analyses are summarized in Table 3-17. The composite burnups for the $(\mathrm{Th}, \mathrm{U}) \mathrm{C}_{2}$ fissile particle and for the total fuel have been calculated from the fissile and fertile burnups using the equation

$$
F_{c}=F_{5} \cdot X+F_{3}(1-X),
$$

where $F_{c}=$ composite burnup,

$\mathrm{F}_{5}=$ fissile burnup from analyses of $\mathrm{UC}_{2}$ particles, $\mathrm{F}_{3}=$ fertile burnup from analyses of $\mathrm{ThC}_{2}$ particles,

\footnotetext{
* The power history shown is from the 335 time interval history used for the "detailed" GAUGE analysis of FSV cycle 1. The hour-by-hour power history exhibited far more variations in power.$$
{ }^{* *} \mathrm{~T}_{\mathrm{C}}=\mathrm{T}_{\text {coolant }}+\mathrm{f}\left(\mathrm{T}_{\text {fuel }}-\mathrm{T}_{\text {coolant }}\right) ; \mathrm{f}=0.62 \text {. }
$$ 
and

$$
\mathrm{X}=\frac{\mathrm{U}_{0}}{\mathrm{U}_{\mathrm{o}}+\mathrm{Th}_{\mathrm{o}}},
$$

where $\mathrm{U}_{\mathrm{O}}=$ appropriate initial uranium loading (atoms), $\mathrm{Th}_{\mathrm{o}}=$ appropriate initial thorium loading (atoms).

Initial heavy metal loadings were obtained from the fuel accountability (Table 3-10).

In addition to the above burnup analyses, fuel burnup was also measured via gamma spectrometry. As part of the gamma spectroscopic examination of the intact fuel element (see Section 3.2), all six pairs of fuel stacks occupying the corner fuel holes were scanned. Later, upon removal of the fuel from the element, each of these 12 fuel stacks was scanned individually. The stacks were placed in thin-walled plexiglass tubes and scanned rod-by-rod as they were moved slowly past the collimator. Absolute calibration of the gamma scanning system using a Cs-137 standard permitted fuel burnup to be determined for the fuel stacks. Burnup data obtained from gamma spectrometry are presented in Table 3-18. Since gamma spectrometry cannot distinguish between the components of an aggregate sample, only the composite burnup for the aggregate (in this case, fuel rods) was determined. However, the composite burnup could be divided into fissile and fertile particle burnup if the fraction of fissions occurring in each type of particle were accurately known from some other source.

Examination of the burnups determined by gamma spectroscopy and by destructive techniques yields the following conclusions:

1. The relative difference between the burnups determined from the gamma scanning of single fuel stacks after removal from the 
element and the burnups determined from scanning of the fuel while still in the element is $\pm 5.6 \%$ ( $1 \sigma$ ) with a bias of $1.9 \%$. The bias is not statistically significant.

2. The relative difference between the element average composite burnup determined from gamma spectrometry (1.38\%) and from destructive measurements $(1.42 \% \pm 0.03 \%)$ is $2.8 \% \pm 2.1 \%(1 \sigma)$.

These results are important because they verify the calibration of the gamma scanning system and demonstrate the validity of gamma scanning as a means of inexpensively acquiring data for fuel burnup (and therefore power generation) in an HTGR fuel element. As part of the FSV surveillance program, gamma spectrometric examinations of irradiated fuel elements in the hot service facility at FSV are planned after each reload, starting with reload 3. These examinations will be performed using a gamma scan robot system currently being developed at GA. This system was successfully employed, in a preliminary state of development, to examine fuel element 1-0743 in the hot cell at GA.

Measured and calculated element average burnups for fuel element 1-0743 are compared in Table 3-19. The relative differences between calculated and measured composite burnups (indicative of total power generation) are $-3.5 \% \pm 2.0 \%{ }^{*}(1 \sigma)$ for the SURVEY-detailed GAUGE analysis, $-9.9 \% \pm 1.9 \%$ ( $\left.1 \sigma\right)$ for the GATT analysis, and $-17.6 \% \pm 1.7 \%(1 \sigma)$ for burnups calculated using fluxes from the FEVER analysis. In each case, the fissile particle burnup is somewhat better predicted than the fertile particle burnup.

A comparison of measured and calculated uranium isotopic concentrations in the $\mathrm{UC}_{2}$ fissile particles irradiated in the burnup monitors is given in Table 3-20. The U-234 and U-235 concentrations are slightly overpredicted

\footnotetext{
* The uncertainties in the relative differences are based on the measurement uncertainties only. The relative difference is given by (Calc - Meas)/Meas, so a negative value means that the calculated burnup is less than the measured burnup.
} 
and the U-236 and U-238 concentrations are underpredicted. This result is as expected, since it has already been observed that the burnup was underpredicted. 
TABLE 3-1

ENVELOPE AND TIME-AVERAGED TEMPERATURES FOR FSV FUEL ELEMENT 1-0743 ELEMENT AVERAGE

TEMPERATURE ENVELOPE

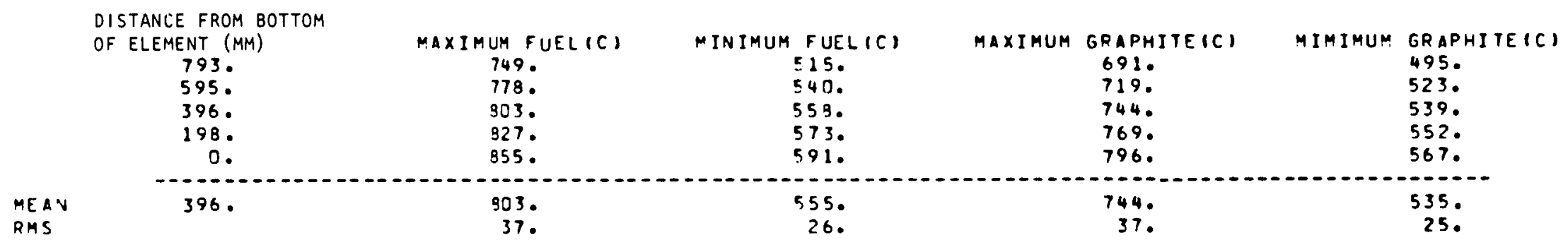

TIME WEIGHTED IRRADIATION TEMPERATURES

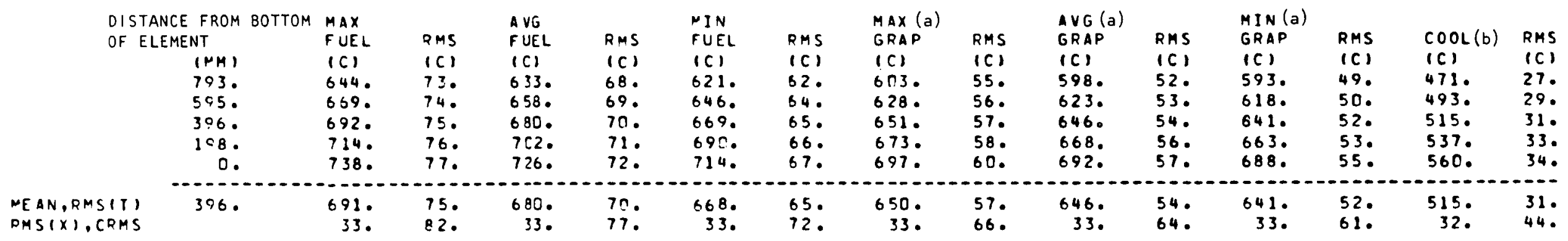

(a) GRAP = GRAPHITE

(b) COOL $=$ COOLANT 
TABLE 3-2

ENVELOPE AND TIME-AVERAGED TEMPERATURES FOR FSV FUEL ELEMENT 1-0743

SURVEY LOCAL POINT $1^{\text {(a) }}$

TEMPERATURE ENVELOPE

\begin{tabular}{|c|c|c|c|c|c|c|c|}
\hline & $\begin{array}{c}\text { DISTANCE FROM BOTTOM } \\
\text { OF ELEMENT (MM) } \\
793 . \\
595 . \\
396 . \\
198 . \\
9 .\end{array}$ & $\begin{array}{c}\text { MAXIMUM FUELIC } \\
749 . \\
779^{\circ} \\
803 . \\
828^{\circ} \\
856 .\end{array}$ & $\begin{array}{l}\text { MINIMUM FUEL ICI } \\
517 . \\
544 . \\
560^{\circ} \\
5750^{\circ} \\
593 .\end{array}$ & MAXIMUM & $\begin{array}{l}\text { GRAPHITE(C) } \\
691 . \\
719 . \\
744 . \\
769 . \\
797 .\end{array}$ & MIMIMUM & $\begin{array}{l}\text { GRAPHITE (C) } \\
498 . \\
525 . \\
539 . \\
552 . \\
567 .\end{array}$ \\
\hline $\begin{array}{l}\text { MEAN } \\
\text { RMS }\end{array}$ & 396. & 803 & $\begin{array}{r}558 . \\
26 .\end{array}$ & & $\begin{array}{r}744 \\
37\end{array}$ & & $\begin{array}{r}536 . \\
24 .\end{array}$ \\
\hline
\end{tabular}

TIME WEIGHTED IRRAOIATION TEMPERATURES

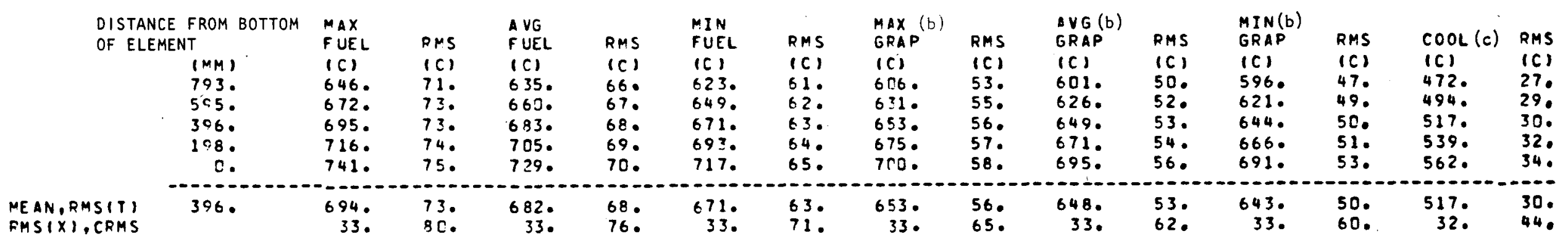

(a) SEE FIG. 3-2

(b) GRAP = GRAPHITE 
TABLE 3-3

ENVELOPE AND TIME-AVERAGED TEMPERATURES FOR FSV FUEL ELEMENT 1-0743 SURVEY LOCAL POINT $2^{\text {(a) }}$

TEMPERATURE ENVELOPE

\begin{tabular}{|c|c|c|c|c|c|c|c|}
\hline & $\begin{array}{l}\text { DISTANCE FROM BOTTOM } \\
\text { OF ELEMENT (MM) } \\
793 . \\
595 . \\
396 . \\
198 . \\
0 .\end{array}$ & $\begin{array}{c}\text { MAXIMUH FUEL(C) } \\
741^{\circ} \\
7690^{\circ} \\
794 . \\
218 . \\
846 .\end{array}$ & $\begin{array}{l}\text { MINIMUM FUEL (C) } \\
439 . \\
455 . \\
469 . \\
482 . \\
497 .\end{array}$ & MAXIMUM & $\begin{array}{l}\text { GRAPHITE(C) } \\
683 . \\
711 . \\
736 \\
760 . \\
788 .\end{array}$ & MI MI MUM & $\begin{array}{l}\text { GRAPHITE (C) } \\
437 . \\
452 . \\
466 . \\
479 . \\
495 .\end{array}$ \\
\hline $\begin{array}{l}\text { MEAN } \\
\text { RMS }\end{array}$ & 396. & $\begin{array}{r}794 . \\
37 .\end{array}$ & $\begin{array}{r}468 \\
20 .\end{array}$ & & $\begin{array}{r}736 \\
37\end{array}$ & & $\begin{array}{r}466 \\
20\end{array}$ \\
\hline
\end{tabular}

TIME WEIGHTED IRRADIATION TEMPERATUPES

\begin{tabular}{|c|c|c|c|c|c|c|c|c|c|c|c|c|c|c|c|}
\hline $\begin{array}{l}\text { DISTAN } \\
\text { OF ELE }\end{array}$ & $\begin{array}{l}\text { FROM BOTTOM } \\
\text { VT }\end{array}$ & $\begin{array}{l}\text { MAX } \\
\text { FUEL }\end{array}$ & RMS & $\begin{array}{l}\text { A VG } \\
\text { F UEL }\end{array}$ & RMS & $\begin{array}{l}\text { MIN } \\
\text { FUEL }\end{array}$ & RMS & $\begin{array}{l}\operatorname{MAX}(b \\
\text { GRAP }\end{array}$ & RMS & $\begin{array}{l}\text { A VG (b) } \\
\text { GRAP }\end{array}$ & RMS & $\begin{array}{l}\operatorname{MIN}(b) \\
\text { GRAP }\end{array}$ & RMS & $\operatorname{coOL}(c)$ & RMS \\
\hline & $\begin{array}{l}(M M) \\
793 . \\
595 . \\
396 . \\
198 . \\
0 .\end{array}$ & $\begin{array}{l}(0) \\
616 . \\
639 . \\
660 . \\
679 . \\
701 .\end{array}$ & $\begin{array}{l}101 \\
96 . \\
100 . \\
103 . \\
106 . \\
109 .\end{array}$ & $\begin{array}{l}(c) \\
675 . \\
628 . \\
648 . \\
668 . \\
690 .\end{array}$ & $\begin{array}{l}101 \\
90 . \\
94 . \\
97 . \\
100 . \\
103 .\end{array}$ & $\begin{array}{l}101 \\
594 . \\
617 . \\
637 . \\
657 . \\
678 .\end{array}$ & $\begin{array}{l}101 \\
85 . \\
88 . \\
91 . \\
94 . \\
97 .\end{array}$ & $\begin{array}{l}(0) \\
577 . \\
600 . \\
620 . \\
640 . \\
662 .\end{array}$ & $\begin{array}{l}101 \\
76 . \\
80 . \\
83 . \\
86 . \\
89 .\end{array}$ & $\begin{array}{l}101 \\
573 . \\
595 . \\
616 . \\
635 . \\
657 .\end{array}$ & $\begin{array}{l}101 \\
73 . \\
77 . \\
80 . \\
83 . \\
87 .\end{array}$ & $\begin{array}{l}(C) \\
568 . \\
591 . \\
611 . \\
631 . \\
653 .\end{array}$ & $\begin{array}{l}101 \\
70 . \\
74 . \\
77 . \\
80 . \\
84 .\end{array}$ & $\begin{array}{l}(C) \\
456 . \\
476 . \\
496 . \\
516 . \\
536 .\end{array}$ & $\begin{array}{l}(C) \\
39 . \\
42 . \\
46 . \\
49 . \\
52 .\end{array}$ \\
\hline $\begin{array}{l}\text { MEAN,RMS (T) } \\
\text { RMS }(X), \text { CRMS }\end{array}$ & 396. & $\begin{array}{l}659 . \\
30 .\end{array}$ & $\begin{array}{l}103 . \\
107 .\end{array}$ & $\begin{array}{r}648 . \\
30 .\end{array}$ & $\begin{array}{r}97 . \\
101 .\end{array}$ & $\begin{array}{r}637 . \\
29 .\end{array}$ & 91. & $\begin{array}{l}620 . \\
30 .\end{array}$ & $\begin{array}{l}83 . \\
88 .\end{array}$ & $\begin{array}{l}615 . \\
30 .\end{array}$ & $\begin{array}{l}80 . \\
85 .\end{array}$ & $\begin{array}{l}611 . \\
30 .\end{array}$ & $\begin{array}{l}77 . \\
83 .\end{array}$ & $\begin{array}{r}496 . \\
28 .\end{array}$ & $\begin{array}{l}46 . \\
54 .\end{array}$ \\
\hline
\end{tabular}
(a) SEE FIG. 3-2
(b) GRAP = GRAPHITE
(c) $\mathrm{COOL}=$ COOLANT 
TABLE 3-4

ENVELOPE AND TIME-AVERAGED TEMPERATURES FOR FSV FUEL ELEMENT 1-0743

SURVEY LOCAL POINT $3^{(a)}$

TEMPERATURE ENVELOPE

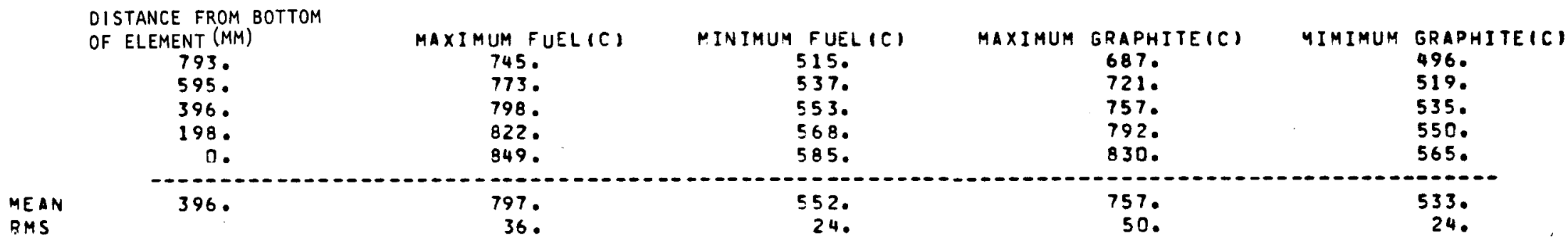

IIME WEIGHTEO IRRADIATION TEMPERATURES

\begin{tabular}{|c|c|c|c|c|c|c|c|c|c|c|c|c|c|c|c|}
\hline $\begin{array}{l}\text { DISTAN } \\
\text { OF ELE }\end{array}$ & $\begin{array}{l}\text { FROM BOTTOM } \\
\text { NT }\end{array}$ & $\begin{array}{l}\text { MAX } \\
\text { FUEL }\end{array}$ & RMS & $\begin{array}{l}\text { A VG } \\
\text { F UEL }\end{array}$ & RMS & $\begin{array}{l}\text { MIN } \\
\text { FUEL }\end{array}$ & RMS & $\begin{array}{l}\operatorname{MAX}(\mathrm{b}) \\
\text { GRAP }\end{array}$ & RMS & $\begin{array}{l}\text { AVG (b) } \\
\text { GRAP }\end{array}$ & RMS & $\begin{array}{l}\operatorname{MIN}(b) \\
\text { GRAP }\end{array}$ & RMS & $\operatorname{coOL}(c)$ & RMS \\
\hline & $\begin{array}{l}(M M) \\
793 . \\
595 \\
396 \\
198 . \\
C .\end{array}$ & $\begin{array}{l}(0) \\
647 . \\
672 . \\
695 . \\
717 . \\
742 .\end{array}$ & $\begin{array}{l}101 \\
64 . \\
65 . \\
65 . \\
66 . \\
67 .\end{array}$ & $\begin{array}{l}(0) \\
635 . \\
661 . \\
684 . \\
706 . \\
731 .\end{array}$ & $\begin{array}{l}101 \\
59 . \\
60 . \\
61 . \\
61 . \\
62 .\end{array}$ & $\begin{array}{l}(0) \\
624 . \\
650 . \\
672 . \\
694 . \\
719 .\end{array}$ & $\begin{array}{l}(0) \\
55 . \\
56 . \\
56 . \\
57 . \\
58 .\end{array}$ & $\begin{array}{l}101 \\
606 . \\
632 . \\
655 . \\
677 . \\
771 .\end{array}$ & $\begin{array}{l}101 \\
48 . \\
49 . \\
50 . \\
51 . \\
52 .\end{array}$ & $\begin{array}{l}(C) \\
601 . \\
627 . \\
650 . \\
672 . \\
697 .\end{array}$ & $\begin{array}{l}101 \\
45 . \\
46 . \\
47 . \\
48 . \\
50 .\end{array}$ & $\begin{array}{l}(C) \\
596 . \\
622 . \\
645 . \\
667 . \\
692 .\end{array}$ & $\begin{array}{l}(c) \\
420^{\circ} \\
440^{\circ} \\
450^{\circ} \\
46 . \\
48 .\end{array}$ & $\begin{array}{l}(C) \\
473 . \\
495 . \\
518 . \\
540 . \\
563 .\end{array}$ & $\begin{array}{l}101 \\
25 . \\
27 . \\
29 . \\
31 . \\
33 .\end{array}$ \\
\hline RMS $(x)$, CRMS & 356. & $\begin{array}{r}695 . \\
33 .\end{array}$ & 73. & $\begin{array}{l}633 . \\
33 .\end{array}$ & $\begin{array}{l}61 . \\
69 .\end{array}$ & $\begin{array}{r}672 . \\
33 .\end{array}$ & $\begin{array}{l}56 . \\
65 .\end{array}$ & $\begin{array}{r}654 \\
33 .\end{array}$ & $\begin{array}{l}50 . \\
60 .\end{array}$ & $\begin{array}{r}649 . \\
33 .\end{array}$ & $\begin{array}{l}47 . \\
58 .\end{array}$ & $\begin{array}{r}644 . \\
33 .\end{array}$ & $\begin{array}{l}45 . \\
56 .\end{array}$ & $\begin{array}{r}518 . \\
32\end{array}$ & $\begin{array}{l}29 . \\
43 .\end{array}$ \\
\hline
\end{tabular}

(a) SEE FIG. 3-2

(b) GRAP = GRAPHITE

(c) $\mathrm{COOL}=$ COOLANT 
TABLE 3-5

ENVELOPE AND TIME-AVERAGED TEMPERATURES FOR FSV FUEL ELEMENT 1-0743

SURVEY LOCAL POINT $4^{(a)}$

TEMDERATURE ENVELOPE

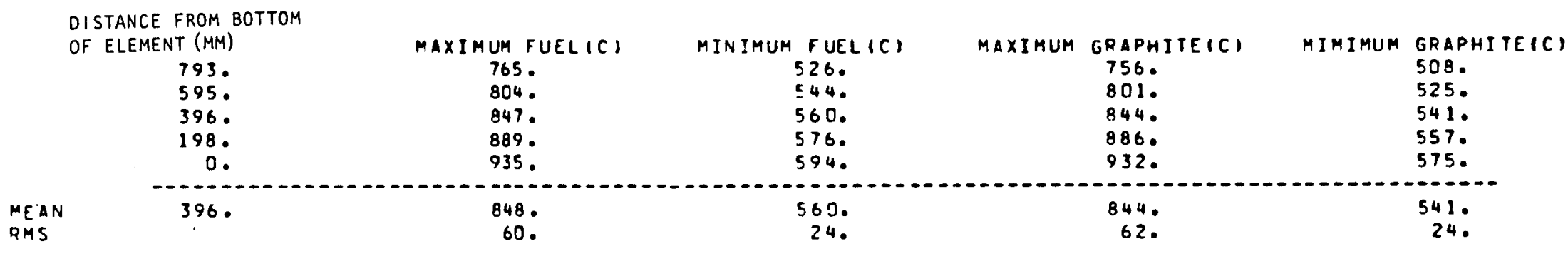

TIME NEIGHTEO IRRAOIATION TEMPERATURES

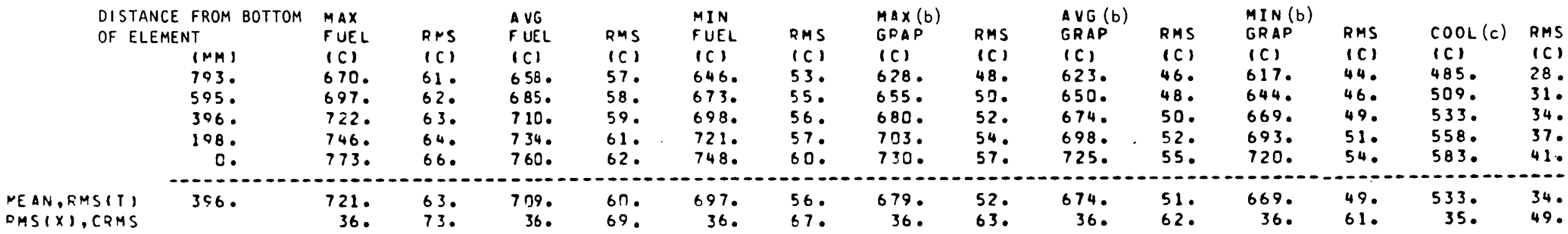

\footnotetext{
(a) SEE FIG. 3-2

(b) GRAP = GRAPHITE

(c) = COOLANT
} 
TABLE 3-6
ENVELOPE AND TIME-AVERAGED TEMPERATURES FOR FSV FUEL ELEMENT 1-0743

SURVEY LOCAL POINT $5^{(a)}$

TEMPERATURE ENVELOPE

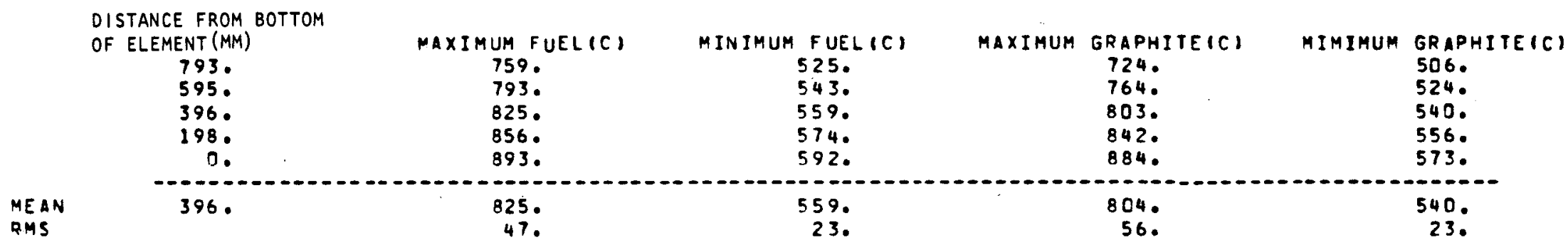

TIME WEIGHTED IRRADIATION TEMPERATURES

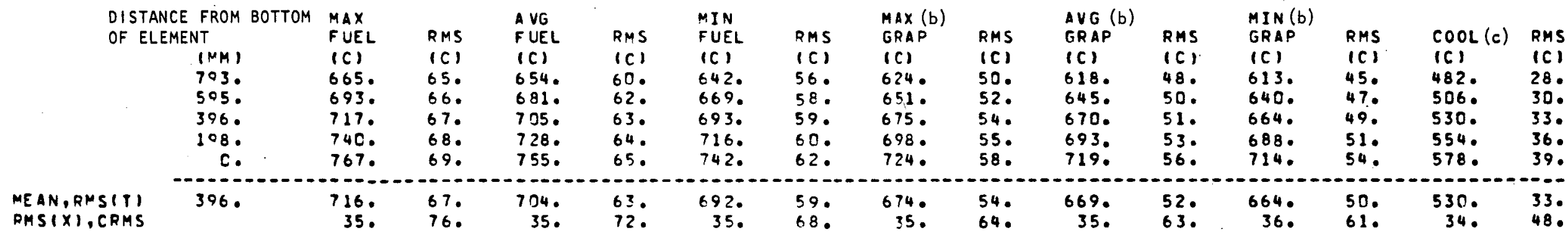

(a) SEE FIG. 3-2

(b) GRAP $=$ GRAPHITE

(c) $\mathrm{COOL}=$ COOLANT 
TABLE 3-7

ENVELOPE AND TIME-AVERAGED TEMPERATURES FOR FSV FUEL ELEMENT 1-0743 SURVEY LOCAL POINT $6^{(a)}$

\section{TEMDERATURE ENVELOPE}

\begin{tabular}{|c|c|c|c|c|c|c|c|}
\hline & $\begin{array}{c}\text { DISTANCE FROM BOTTOM } \\
\text { OF ELEMENT (MM) } \\
793 . \\
595 . \\
396 . \\
198 . \\
3 .\end{array}$ & $\begin{array}{l}\text { MAXIMUM FUEL (C) } \\
745^{\circ} \\
774^{\circ} \\
798 . \\
822 . \\
850^{\circ}\end{array}$ & $\begin{array}{l}\text { MIN IMUM F UEL I C I } \\
506 . \\
527 . \\
547 . \\
566 . \\
583 .\end{array}$ & MAXIMUM & $\begin{array}{l}\text { GRAPHITEICI } \\
687 . \\
715 . \\
740 . \\
764 . \\
791 .\end{array}$ & MIMIMUM & $\begin{array}{l}\text { GRAPHITE(C) } \\
493 . \\
5170 \\
5320 \\
547 . \\
563 .\end{array}$ \\
\hline $\begin{array}{l}\text { MEAN } \\
\text { RMS }\end{array}$ & 396. & $\begin{array}{r}798 . \\
36 .\end{array}$ & $\begin{array}{r}546 . \\
27\end{array}$ & & $\begin{array}{r}739 . \\
36 .\end{array}$ & & $\begin{array}{r}530 . \\
24 .\end{array}$ \\
\hline
\end{tabular}

IIME WEIGHTED IRRADIATION TEMPERATURES

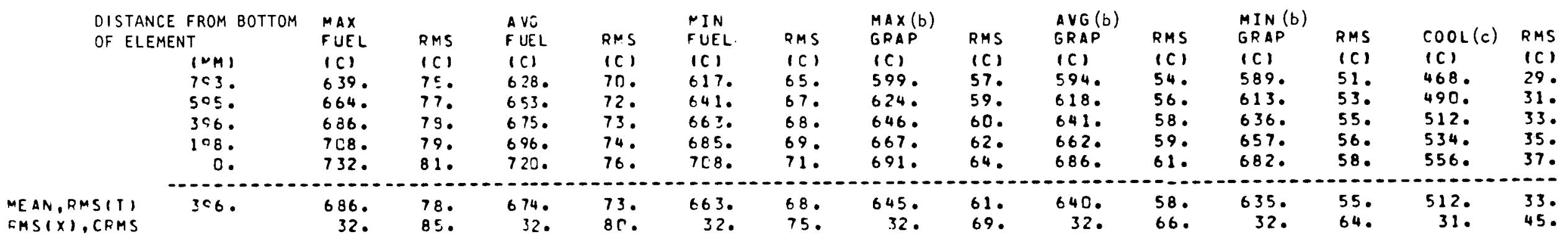

(a) SEE FIG. 3-2

(b) GRAP = GRAPHITE

(c) $\mathrm{COOL}=$ COOLANT 
TABLE 3-8

ENVELOPE AND TIME-AVERAGED TEMPERATURES FOR FSV FUEL ELEMENT 1-0743 SURVEY LOCAL POINT $7^{(a)}$

TEMPERATURE ENVELOPE

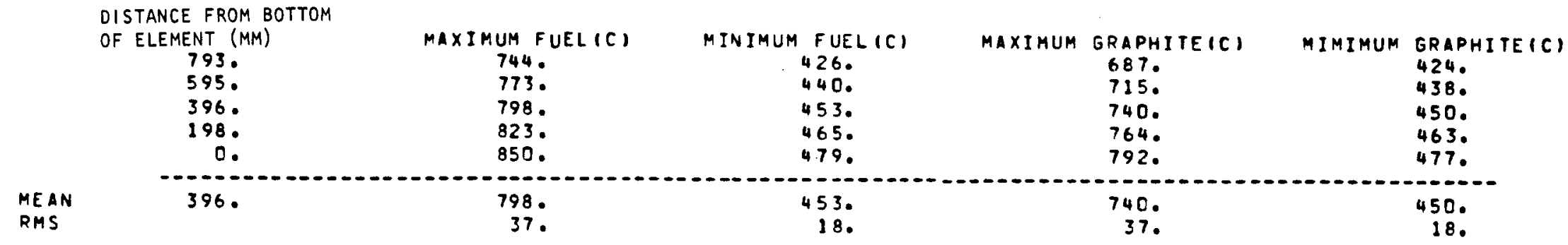

TIME WEIGHTED IRRADIATION TEMPERATURES

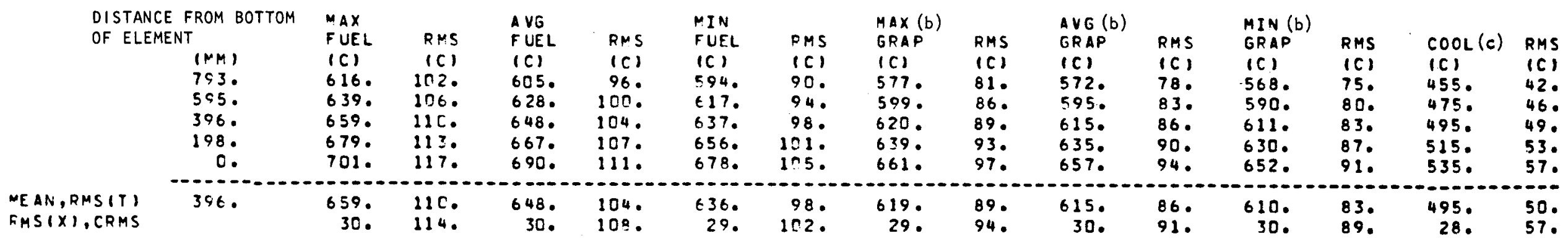
(a) See Fig. 3-2
(b) GRAP = GRAPHIT
(c) COOL $=$ COOLANT 
TABLE 3-9

FAST NEUTRON FLUENCES FOR FSV FUEL ELEMENT 1-0743

\begin{tabular}{c|c|c|c|c|c|c}
\hline \multicolumn{2}{c|}{ Rad1al Location } & \multicolumn{5}{|c}{ Fast Neutron F1uence $\left(10^{25} \mathrm{n} / \mathrm{m}^{2}\right)(\mathrm{E}>29 \mathrm{fJ})_{\mathrm{HTGR}}(\mathrm{a})$} \\
\cline { 2 - 6 } FSV & $\begin{array}{c}\text { SURVEY } \\
\text { Local Point }\end{array}$ & $z=793 \mathrm{~mm}(\mathrm{~b})$ & $z=594.7 \mathrm{~mm}$ & $z=396.5 \mathrm{~mm}$ & $z=198.2 \mathrm{~mm}$ & $z=0 \mathrm{~mm}$ \\
\hline Center & 1 & 0.95 & 0.96 & 0.96 & 0.96 & 0.91 \\
Corner 1 & 4 & 1.09 & 1.10 & 1.10 & 1.10 & 1.04 \\
Corner 2 & 5 & 1.08 & 1.09 & 1.10 & 1.09 & 1.03 \\
Corner 3 & 6 & 0.99 & 1.00 & 1.00 & 0.99 & 0.94 \\
Corner 4 & 7 & 0.83 & 0.83 & 0.84 & 0.83 & 0.79 \\
Corner 5 & 2 & 0.82 & 0.82 & 0.83 & 0.82 & 0.78 \\
Corner 6 & 3 & 0.98 & 0.99 & 0.99 & 0.99 & 0.93 \\
Element & Element & 0.96 & 0.97 & 0.97 & 0.96 & 0.91 \\
average & average & & & & & 0.96 \\
\hline
\end{tabular}

(a) From SURVEY-detailed GAUGE analyses.

(b) Axial location relative to bottom of element. 
TABLE 3-10

FUEL ACCOUNTABILITY FOR FSV FUEL ELEMENT 1-0743

\begin{tabular}{|c|c|c|c|}
\hline \multirow[b]{2}{*}{ Particle } & \multirow[b]{2}{*}{ Nuclide } & \multicolumn{2}{|c|}{ Heavy Meta 1 Weight } \\
\hline & & Initial & Current \\
\hline Fertile & Th-232 & 10827.37 & 10680.89 \\
\hline Fertile & $\mathrm{Pa}-231$ & 0.00 & 0.03 \\
\hline Fertile & $\mathrm{U}-232$ & 0.00 & 0.01 \\
\hline Fertile & $\mathrm{U}-233$ (a) & 0.00 & 114.24 \\
\hline Fertile & $\mathrm{U}-234$ & 0.00 & 5.02 \\
\hline Fertile & $\mathrm{U}-235$ & 0.00 & 0.28 \\
\hline Fertile & $\mathrm{U}-236$ & 0.00 & 0.01 \\
\hline Fissile & $\mathrm{Th}-232$ & 1949.63 & 1923.25 \\
\hline Fissile & $\mathrm{Pa}-231$ & 0.00 & 0.01 \\
\hline Fissile & $\mathrm{U}-232$ & 0.00 & 0.00 \\
\hline Fissile & $\mathrm{U}-233(\mathrm{a})$ & 0.00 & 20.57 \\
\hline Fissile & $U-234$ & 3.45 & 3.81 \\
\hline Fissile & $\mathrm{U}-235$ & 433.15 & 263.76 \\
\hline Fissile & $\mathrm{U}-236$ & 1.32 & 31.94 \\
\hline Fissile & $\mathrm{U}-238$ & 27.09 & 25.70 \\
\hline Fissile & $\mathrm{Np}-237$ & 0.00 & 1.01 \\
\hline Fissile & $\mathrm{Pu}-232$ & 0.00 & 0.09 \\
\hline Fissile & $\mathrm{Pu}-239(\mathrm{~b})$ & 0.00 & 0.56 \\
\hline Fissile & $\mathrm{Pu}-240$ & 0.00 & 0.17 \\
\hline Fissile & $\mathrm{Pu}-241$ & 0.00 & 0.10 \\
\hline Fissile & $\mathrm{Pu}-242$ & 0.00 & 0.02 \\
\hline \multicolumn{2}{|l|}{ Total } & 13242.00 & 13071.44 \\
\hline \multicolumn{2}{|c|}{$\begin{array}{l}\text { Total fissile uranium } \\
\text { Total uranium }\end{array}$} & $\begin{array}{l}433.15 \\
465.00\end{array}$ & $\begin{array}{l}398.85 \\
465.33\end{array}$ \\
\hline \multicolumn{2}{|c|}{$\begin{array}{l}\text { Total fissile plutonium } \\
\text { Total plutonium }\end{array}$} & $\begin{array}{l}0.00 \\
0.00\end{array}$ & $\begin{array}{l}0.66 \\
0.93\end{array}$ \\
\hline \multicolumn{2}{|c|}{$\begin{array}{l}\text { Effective U-233 enrichment (\%) } \\
\text { Effective U-235 enrichment (\%) } \\
\text { U-232 ( ppm) }\end{array}$} & $\begin{array}{r}0.00 \\
93.15 \\
0.00\end{array}$ & $\begin{array}{l}28.97 \\
56.74 \\
26.26\end{array}$ \\
\hline \multirow{4}{*}{\multicolumn{2}{|c|}{$\begin{array}{l}\text { Fertile particle FIMA }(\%) \\
\text { Fissile particle FIMA }(\%) \\
\text { Burnup (MWd/tonne) } \\
\text { Cumulative EFPD }\end{array}$}} & 0.00 & 0.25 \\
\hline & & 0.00 & 5.90 \\
\hline & & 0.00 & 12208.26 \\
\hline & & 0.00 & 174.00 \\
\hline
\end{tabular}

(a) Includes full decay of Pa-233.

(b) Includes full decay of $\mathrm{Np}-239$. 
TABLE 3-11

COMPARISON OF MEASURED (Cs-137) AND CALCULATED TIME-AVERAGED

RADIAL POWER DISTRIBUTIONS FOR FSV FUEL ELEMENT 1-0743

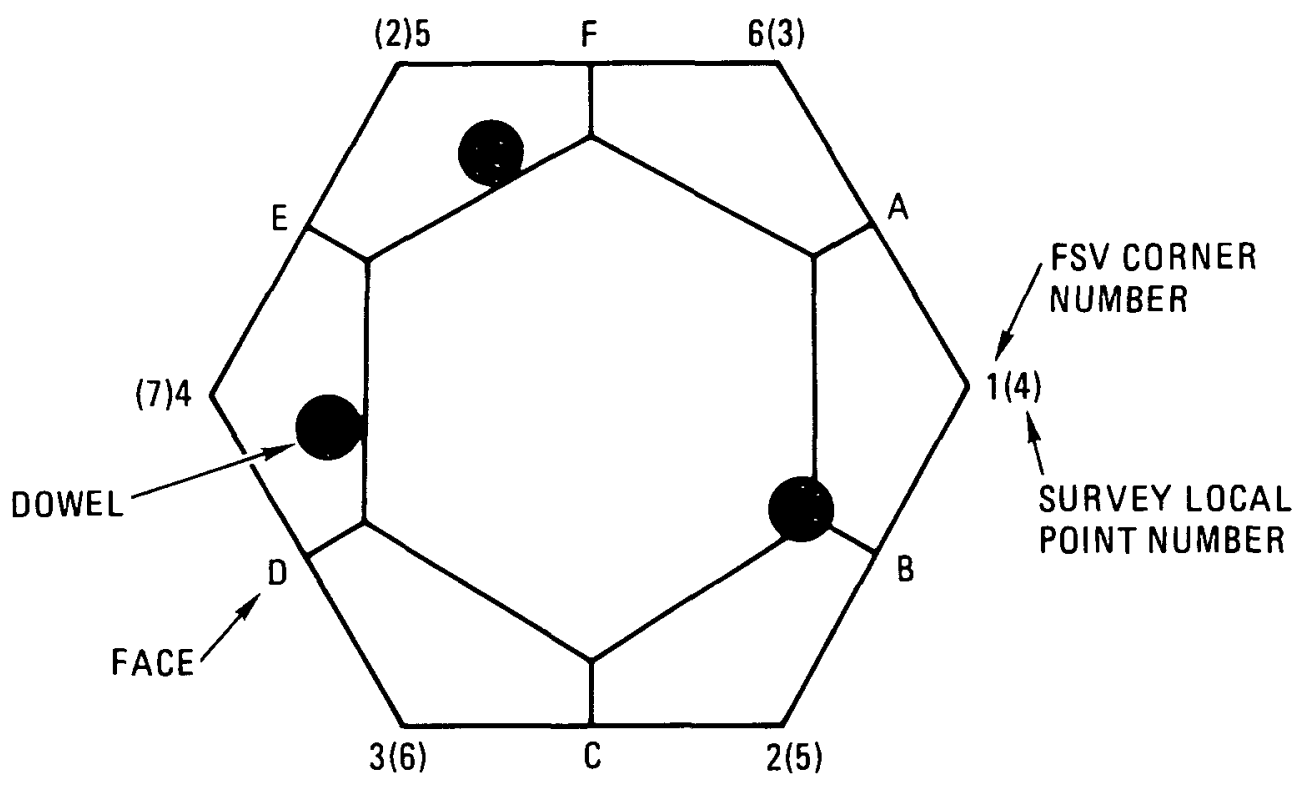

\begin{tabular}{|c|c|c|c|c|c|c|c|}
\hline \multirow[b]{4}{*}{$\begin{array}{l}\text { Portion } \\
\text { of } \\
\text { Element }\end{array}$} & \multicolumn{7}{|c|}{ Normalized Radial Power } \\
\hline & \multirow{2}{*}{\multicolumn{3}{|c|}{ Measured }} & \multicolumn{4}{|c|}{ Calculated } \\
\hline & & & & \multicolumn{2}{|c|}{ Case $I(b)$} & \multicolumn{2}{|c|}{ Case $\operatorname{III}(c)$} \\
\hline & $\begin{array}{l}\text { Number } \\
\text { of Fuel } \\
\text { Stacks }\end{array}$ & $\begin{array}{l}\text { Relative } \\
\text { Power }\end{array}$ & $\pm 1 \sigma(a)$ & $\begin{array}{l}\text { Relative } \\
\text { Power }\end{array}$ & $\begin{array}{c}\frac{\mathrm{Calc}}{\text { Meas }}-1 \\
(\%)\end{array}$ & $\begin{array}{l}\text { Relative } \\
\text { Power }\end{array}$ & $\begin{array}{c}\frac{\mathrm{Calc}}{\text { Meas }}-1 \\
(\%)\end{array}$ \\
\hline Center & 30 & 0.98 & 0.01 & 1.01 & +3.1 & 1.00 & +2.0 \\
\hline Corner 1 & 7 & 1.04 & 0.02 & 1.06 & +1.9 & 0.99 & -4.8 \\
\hline Corner 2 & 7 & 1.06 & 0.02 & 1.05 & -0.9 & 1.00 & -5.7 \\
\hline Corner 3 & 5 & 0.98 & 0.02 & 0.98 & 0 & 1.00 & +2.0 \\
\hline Corner 4 & 5 & 0.97 & 0.02 & 0.93 & $-4 \cdot 1$ & 1.02 & +5.2 \\
\hline Corner 5 & 5 & 1.00 & 0.02 & 0.93 & -7.0 & 1.01 & +1.0 \\
\hline Corner 6 & 7 & 1.05 & 0.02 & 1.01 & -3.8 & 0.98 & -6.7 \\
\hline
\end{tabular}

(a) $\pm 1 \sigma$ error on mean; $\varepsilon=s / \sqrt{n}$, where $s=s t a n d a r d$ deviation and $\mathbf{n}=$ number of fuel stacks.
(b) SURVEY-detailed GAUGE analysis.
(c) GAUGE analysis with 11 -time-interval power history. 
TABLE 3-12

COMPARISON OF MEASURED ( $\mathrm{z}-95$ ) AND CALCULATED RADIAL POWER DISTRIBUTIONS AT EOL FOR FSV FUEL ELEMENT 1-0743

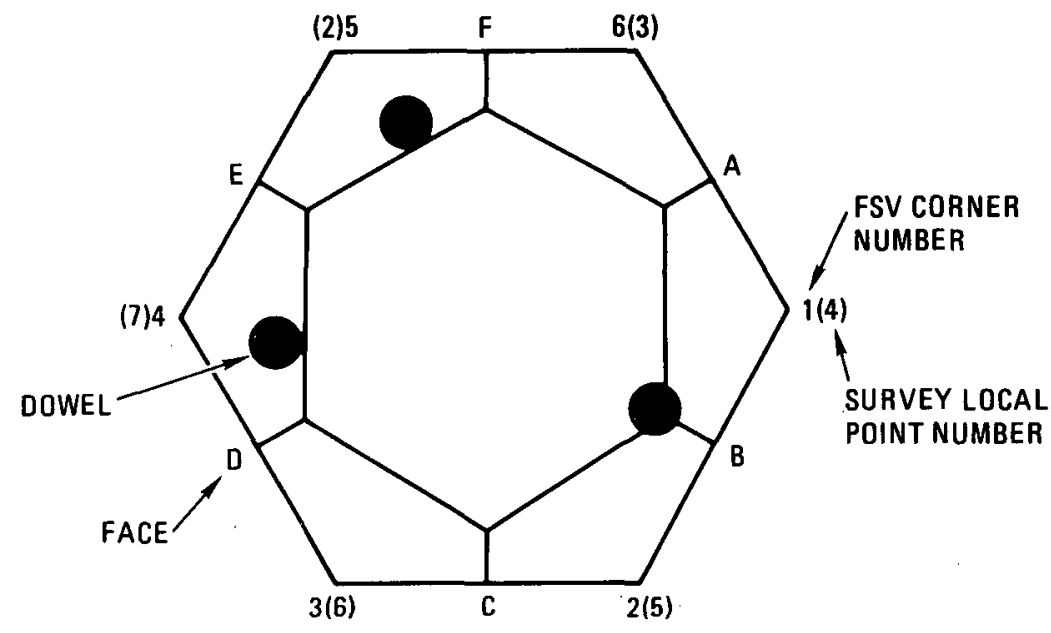

\begin{tabular}{|c|c|c|c|c|c|c|c|c|c|}
\hline \multirow[b]{4}{*}{$\begin{array}{l}\text { Portion } \\
\text { of } \\
\text { Element }\end{array}$} & \multicolumn{9}{|c|}{ Normalized Radial Power } \\
\hline & \multirow{2}{*}{\multicolumn{3}{|c|}{ Measured }} & \multicolumn{6}{|c|}{ Calculated } \\
\hline & & & & \multicolumn{2}{|c|}{ Case I (b) } & \multicolumn{2}{|c|}{ Case II (c) } & \multicolumn{2}{|c|}{ Case $\operatorname{III}(d)$} \\
\hline & $\begin{array}{l}\text { Number } \\
\text { of Fuel } \\
\text { Stacks }\end{array}$ & $\begin{array}{l}\text { Relative } \\
\text { Power }\end{array}$ & $\pm 1 \sigma(a)$ & $\begin{array}{l}\text { Relative } \\
\text { Power }\end{array}$ & $\begin{array}{c}\frac{\text { Calc }}{\text { Meas }}-1 \\
(\%)\end{array}$ & $\begin{array}{l}\text { Relative } \\
\text { Power }\end{array}$ & $\begin{array}{l}\frac{\text { Calc }}{\text { Meas }}-1 \\
(\%)\end{array}$ & $\begin{array}{l}\text { Relative } \\
\text { Power }\end{array}$ & $\frac{\mathrm{Ca} 1 \mathrm{c}}{\text { Meas }}-1$ \\
\hline Center & 30 & 0.98 & 0.01 & 1.00 & +2.0 & 1.00 & +2.0 & 1.00 & +2.0 \\
\hline Corner 1 & 7 & 1.03 & 0.02 & 1.02 & $-1 \cdot 0$ & 1.01 & -1.9 & 1.00 & -2.9 \\
\hline Corner 2 & 7 & 1.04 & 0.01 & 1.02 & -1.9 & 1.01 & -2.9 & 1.02 & -1.9 \\
\hline Corner 3 & 5 & 0.98 & 0.02 & 0.99 & +1.0 & 0.99 & +1.0 & 1.00 & +2.0 \\
\hline Corner 4 & 5 & 0.98 & 0.02 & 0.99 & +1.0 & 1.01 & +3.1 & 1.01 & +3.1 \\
\hline Corner 5 & 5 & 1.02 & 0.01 & 0.98 & -3.9 & 1.00 & -2.0 & 0.99 & -2.9 \\
\hline Corner 6 & 7 & 1.06 & 0.01 & 0.99 & -6.6 & 0.98 & -7.5 & 0.98 & -7.5 \\
\hline
\end{tabular}

(a) $\pm 1 \sigma$ error on mean; $\varepsilon=s / \sqrt{n}$, where $s=$ standard deviation and $n=$ number of fuel stacks.

(b) SURVEY-detalled GAUGE analysis.

(c) GATT analysis with 11-time-interval power history.

(d) GAUGE analysis with 11-time-interval power history. 
TABLE 3-13

DOSIMETER WIRE REACTIONS

\begin{tabular}{c|l|l|l}
\hline $\begin{array}{c}\text { Monitor } \\
\text { Type }\end{array}$ & $\begin{array}{c}\text { Reaction of } \\
\text { Interest }\end{array}$ & $\begin{array}{c}\text { Product } \\
\text { Half-Life }\end{array}$ & \multicolumn{1}{c}{$\begin{array}{c}\text { Neutron } \\
\text { Energy Group }\end{array}$} \\
\hline $\mathrm{V}-\mathrm{Co}, 0.216 \% \mathrm{Co}$ & $5{ }^{9} \mathrm{Co}(n, \gamma)^{60} \mathrm{Co}$ & $5.26 \mathrm{yr}$ & Thermal (0-0.38 aJ) \\
$\begin{array}{c}\mathrm{V}-\mathrm{Fe}, 0.522 \% \mathrm{Fe} \\
(88.24 \% \mathrm{Fe}-54)\end{array}$ & $54 \mathrm{Fe}(n, \rho)^{54 \mathrm{Mn}}$ & 312.1 days & Fast (>29 fJ $)$ \\
$\mathrm{V}$ & $51_{\mathrm{V}(n, \gamma)^{32} \mathrm{~V} \stackrel{\mathrm{B}-}{\rightarrow} 52 \mathrm{Cr}}$ & Stable & Thermal (0-0.38 aJ) \\
\hline
\end{tabular}


TABLE $3-14$

CROSS SECTIONS USED FOR DOSIMETRY CALCULATIONS

\begin{tabular}{c|c}
\hline Reaction & $\begin{array}{c}\text { Cross Section(a) } \\
\text { (barns) }\end{array}$ \\
\hline${ }^{59} \mathrm{Co}(n, \gamma)^{60} \mathrm{Co}$ & 18.9 \\
$51_{\mathrm{V}}(n, \gamma)^{52 \mathrm{~V}}$ & 2.04 \\
${ }^{54} \mathrm{Fe}(n, \gamma)^{54} \mathrm{Mn}$ & 0.0275 \\
${ }^{60} \mathrm{Co}(n, \gamma)^{61} \mathrm{Co}$ & 1.0 \\
${ }^{54} \mathrm{Mn}(n, \gamma)^{55} \mathrm{Mn}$ & 5.8 \\
${ }^{54} \mathrm{Fe}(n, \gamma)^{55} \mathrm{Fe}$ & 1.13 \\
\hline
\end{tabular}

(a) Cross section obtained from Ref. 12 . 
TABLE 3-15

COMPARISON OF CALCULATED AND MEASURED NEUTRON FLUENCE FOR FSV FUEL ELEMENT 1-0743

\begin{tabular}{|c|c|c|c|c|c|c|c|c|c|c|c|}
\hline \multirow[b]{2}{*}{ Dosimeter } & \multirow[b]{2}{*}{$\begin{array}{l}\text { Monl cor } \\
\text { Number }\end{array}$} & \multicolumn{2}{|c|}{ Location in Element } & \multirow[b]{2}{*}{$\begin{array}{l}\text { Neutron } \\
\text { Group }\end{array}$} & \multicolumn{3}{|c|}{$\begin{array}{c}\text { Calculated } \\
\text { Fluence } \\
\left(10^{25} \mathrm{n} / \mathrm{m}^{2}\right)(E>29 \mathrm{fJ})_{\mathrm{HTGR}}\end{array}$} & \multirow{2}{*}{$\begin{array}{l}\text { Measured } \\
\text { Fluence } \\
\left(\times 10^{25} \mathrm{n} / \mathrm{m}^{2}\right)\end{array}$} & \multicolumn{3}{|c|}{$\begin{array}{l}\text { Relative Difference } \\
\frac{\text { Calc }}{\text { Meas }}-1 \\
(\%)\end{array}$} \\
\hline & & $\begin{array}{l}\text { Stack } \\
\text { Number }\end{array}$ & $\left(1 n_{0}\right)(a)^{\text {Bock }}$ & & Case I(b) & Case II (c) & Case III(d) & & Case I(b) & Case II (c) & Case III (d) \\
\hline v & $\begin{array}{l}21 \\
22 \\
81 \\
82 \\
\text { Av }\end{array}$ & $\begin{array}{r}12 \\
12 \\
278 \\
278 \\
--\end{array}$ & $\begin{array}{r}4.8 \\
25.2 \\
4.8 \\
25.2 \\
--\end{array}$ & Thermal & $\mathrm{ND}(e)$ & ND & $1.93(f)$ & $\begin{array}{l}1.40 \\
1.38 \\
1.33 \\
1.41 \\
1.38\end{array}$ & & & +39.9 \\
\hline $\mathrm{v}-\mathrm{Co}$ & $\begin{array}{l}21 \\
22 \\
81 \\
82 \\
\text { Av }\end{array}$ & $\begin{array}{r}12 \\
12 \\
278 \\
278 \\
--\end{array}$ & $\begin{array}{r}4.8 \\
25.2 \\
4.8 \\
25.2 \\
--\end{array}$ & Thermal & ND & $\mathrm{ND}$ & $1.93(f)$ & $\begin{array}{l}2.09 \\
2.19 \\
2.24 \\
2.26 \\
2.19\end{array}$ & & & -11.9 \\
\hline $\mathrm{V}-\mathrm{Fe}$ & $\begin{array}{l}21 \\
22 \\
81 \\
82 \\
\text { Av }\end{array}$ & $\begin{array}{r}12 \\
12 \\
278 \\
278 \\
--\end{array}$ & $\begin{array}{r}4.8 \\
25.2 \\
4.8 \\
25.2 \\
--\end{array}$ & Fast & $\begin{array}{l}0.81 \\
0.83 \\
1.07 \\
1.09 \\
0.95(g)\end{array}$ & $0.91(f)$ & $0.94(f)$ & $\begin{array}{l}0.84 \\
0.88 \\
1.03 \\
1.06 \\
0.95\end{array}$ & $\begin{array}{c}-3.6 \\
-5.7 \\
+3.9 \\
+2.8 \\
-0.7 \pm 4.7(h)\end{array}$ & -4.2 & -1.1 \\
\hline
\end{tabular}

(a) 1 1n. $=25.4 \mathrm{~mm}$.

(b) SURVEY-detalled GAUGE analysis.

(c) GATT analysis with 11-time-1nterval power history.

(d) GAUGE analysis with 11-time-1ntervals (column average fluxes) and GATT analysis (axial flux factors). Values are taken from Ref. 3-9.

(e) $\mathrm{ND}=$ not determined.

(f) Element average fluence.

(g) Shown for comparison only. Not used to calculate average relative difference.

(h) Mean difference and standard deviation. 
TABLE $3-16$

COMPARISON OF MEASURED AND CALCULATED TEMPERATURES FOR SiC PELLETS IRRADIATED IN FSV FUEL ELEMENT 1-0743

\begin{tabular}{|c|c|c|c|c|c|c|c|}
\hline $\begin{array}{l}\text { Monftor } \\
\text { ID }\end{array}$ & $\begin{array}{l}\text { Fuel } \\
\text { Stack }\end{array}$ & $\begin{array}{c}\text { Axial } \\
\text { Position } \\
\text { (cm from bottom) }\end{array}$ & $\begin{array}{l}\text { Annealing Curve } \\
\text { Intersection } \\
\text { Temperature } \\
\left({ }^{\circ} \mathrm{C}\right)\end{array}$ & $\begin{array}{l}\text { Measured (a) } \\
\text { Irradiation } \\
\text { Temperature } \\
\quad\left({ }^{\circ} \mathrm{C}\right)\end{array}$ & $\begin{array}{l}95 \% \text { Confidence Limits }(\mathrm{a}) \\
\text { for Measured } \\
\text { Irradiation Temperature } \\
\left({ }^{\circ} \mathrm{C}\right)\end{array}$ & $\begin{array}{l}\text { Calculated }(\mathrm{b}) \\
\text { Temperature } \\
\qquad\left({ }^{\circ} \mathrm{C}\right)\end{array}$ & $\begin{array}{l}\text { Difference } \\
\mathrm{T}_{\mathrm{C}}-\mathrm{T}_{\mathrm{M}} \\
\left({ }^{\circ} \mathrm{C}\right)\end{array}$ \\
\hline 21 & 12 & 12 & 755 & 704 & $674<\mathrm{T}<737$ & 728 & +24 \\
\hline 22 & 12 & 64 & 720 & 648 & $615<T<683$ & 668 & +20 \\
\hline 81 & 278 & 12 & 758 & 707 & $677<\mathrm{T}<740$ & 737 & +30 \\
\hline 82 & 278 & 64 & 723 & 651 & $618<\mathrm{T}<686$ & 675 & +24 \\
\hline Average & - & -- & -- & -- & -- & -- & $+24 \pm 4$ \\
\hline
\end{tabular}

(a) Irradiation temperatures determined from annealing curve intersection temperatures using the calibration curve for SiC temperature monitors presented in Ref. 13.

(b) Temperatures obtained from SURVEY-calculated peak fuel and coolant temperatures at the axial locations of the

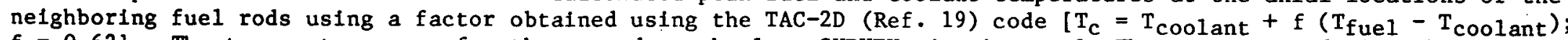
$f=0.62]$. The temperatures are for the second to the last SURVEY time interval. The core power during this interval was $546 \mathrm{MW}$, and the temperatures are representative of the highest temperatures over the last $1 \mathrm{x} 10^{20}$ $\mathrm{n} / \mathrm{cm}^{2}$ (E > $\left.29 \mathrm{fJ}\right)_{\mathrm{HTGR}}$. 
TABLE 3-17

BURNUP MEASUREMENTS FOR FSV FUEL ELEMENT 1-0743 USING DESTRUCTIVE TECHNIQUES

\begin{tabular}{|c|c|c|c|c|c|c|c|c|c|c|c|c|c|c|c|c|}
\hline \multirow{4}{*}{$\begin{array}{l}\text { Burnup (a) } \\
\text { Monitor }\end{array}$} & \multicolumn{6}{|c|}{ F18s1le Burnup } & \multicolumn{6}{|c|}{ Fertile Burnup } & \multirow{2}{*}{\multicolumn{2}{|c|}{$\begin{array}{c}\text { (Th, U) })_{2}(\mathrm{c}) \\
\text { Burnup }\end{array}$}} & \multirow{2}{*}{\multicolumn{2}{|c|}{$\begin{array}{c}\text { Composite(c) } \\
\text { Burnup }\end{array}$}} \\
\hline & \multirow{3}{*}{$\begin{array}{l}\text { Sample } \\
\text { No. }\end{array}$} & \multirow{3}{*}{$\begin{array}{l}\text { Axta1 } \\
\text { Location } \\
(\mathrm{cm})(\mathrm{b})\end{array}$} & \multicolumn{4}{|c|}{ FIMA (\%) } & \multirow{3}{*}{$\begin{array}{c}\text { Fuel } \\
\text { Rod } \\
\text { (Stack-Rod) }\end{array}$} & \multirow{3}{*}{$\begin{array}{c}\text { Sample e } \\
\text { No. }\end{array}$} & \multirow{3}{*}{$\begin{array}{l}\text { Axtal } \\
\text { Location } \\
\text { (cm)(b) }\end{array}$} & \multicolumn{3}{|c|}{ FIMA $(\%)$} & & & & \\
\hline & & & \multirow{2}{*}{$\begin{array}{l}\text { Radio- } \\
\text { chemt atry } \\
\text { Method }\end{array}$} & \multirow{2}{*}{$\begin{array}{c}\text { Mass } \\
\text { Spectometric } \\
\text { Method } \\
\end{array}$} & \multicolumn{2}{|c|}{ Al1 Measurements } & & & & \multirow{2}{*}{$\begin{array}{l}\text { Individual } \\
\text { Particles }\end{array}$} & \multicolumn{2}{|c|}{ All Measurements } & \multirow{2}{*}{\begin{tabular}{|l} 
FIMA \\
$(\%)$
\end{tabular}} & \multirow{2}{*}{$\begin{array}{c} \pm 1 \sigma(d) \\
(\%)\end{array}$} & \multirow{2}{*}{$\begin{array}{l}\text { FIMA } \\
\text { (z) }\end{array}$} & \multirow{2}{*}{$\begin{array}{c} \pm 10(d) \\
(z)\end{array}$} \\
\hline & & & & & Avg. & Std. Dev. & & & & & Avg. & Std. Dev. & & & & \\
\hline \multirow[t]{3}{*}{21} & 4 & 12.2 & 32.1 & 30.2 & & & $12-4$ & 1 & 20.7 & 0.30 & & & & & & \\
\hline & 5 & & 32.2 & 30.8 & 31.3 & \pm 1.0 & & 2 & & 0.31 & 0.30 & \pm 0.01 & 6.27 & 0.19 & 1.38 & 0.04 \\
\hline & & & & & & & & 8 & & 0.30 & & & & & & \\
\hline \multirow{2}{*}{22} & 4 & & 31.6 & 30.1 & 30.9 & 50.8 & & 4 & & 0.32 & 0.32 & \pm 0.01 & 6.21 & 0.15 & 1.38 & 0.03 \\
\hline & & & & & & & & 5 & & 0.33 & & & & & & \\
\hline \multirow[t]{3}{*}{81} & 4 & 12.2 & 33.7 & 32.8 & & & $279-3$ & 2 & 12.2 & 0.35 & & & & & & \\
\hline & 5 & & 31.6 & 31.1 & 32.3 & \pm 1.2 & & 6 & & 0.33 & 0.34 & \pm 0.01 & 6.49 & 0.23 & 1.45 & 0.04 \\
\hline & & & & & & & & 8 & & 0.35 & & & & & & \\
\hline Element avo & $=\operatorname{rage}(0)$ & & & & & & & & & & 0.32 & \pm 0.01 & 6.38 & 0.15 & 1.42 & 0.03 \\
\hline
\end{tabular}

(a) Monitors 21 and 22 were in fuel stack 12 and monitor 81 was in fuel stack 278 .

(b) Centimeters from bottom of element.

(c) $(T h, U) C_{2}$ burnup $-F_{C}=\left(F_{5}\right)(X)+\left(F_{3}\right)(1-X)$, where $F_{5}=$ fissfle burnup, $F_{3}=$ fertlle burnup, and $x=U_{0} /\left(U_{0}+T h_{0}\right)$. $U_{0}$ and $T h_{0}$ are the Initial heavy metal loadings.

(d) $\mathrm{dF}_{\mathrm{C}}=\left[\left(\partial \mathrm{F}_{\mathrm{c}} / \partial \mathrm{F}_{5}\right)^{2}(\mathrm{dF})^{2}+\left(\partial \mathrm{F}_{\mathrm{c}} / \partial \mathrm{F}_{3}\right)^{2}(\mathrm{dF})^{2}\right]^{1 / 2}=\left[(\mathrm{X})^{2}(\mathrm{dF} 5)^{2}+(1-\mathrm{X})^{2}(\mathrm{dF})^{2}\right)^{2}$. Uncertalnty in heavy metal loadings was omitted because results are to be compared with calculations that assumed the same loadings.

(e) Element average burnups obtalned by averaglng the results at the locations of monitors 21 and 81 . The average neutron flux for these two locations was approximately equivalent to the element average flux. 
TABLE 3-18

BURNUP MEASUREMENTS FOR FSV FUEL ELEMENT 1-0743 USING GAMMA SCANNING

\begin{tabular}{|c|c|c|c|c|c|}
\hline \multirow[b]{3}{*}{$\begin{array}{l}\text { Fuel } \\
\text { Stack }\end{array}$} & \multicolumn{5}{|c|}{ Fuel Stack Average Burnup } \\
\hline & \multirow[b]{2}{*}{$\begin{array}{l}\text { Corner Scans, (a) } \\
\text { Composite } \\
\text { FIMA } \\
(\%)\end{array}$} & \multirow{2}{*}{\multicolumn{2}{|c|}{$\begin{array}{c}\text { Single Stack Scans, (b) } \\
\text { Composite } \\
\text { FIMA } \\
(\%)\end{array}$}} & \multicolumn{2}{|c|}{ Average } \\
\hline & & & & $\begin{array}{l}\text { Composite } \\
\text { FIMA } \\
(\%)\end{array}$ & $\begin{array}{l}\text { Relative Diff, } \\
\frac{\text { Corner }}{\text { Single }}-1 \\
(\%)\end{array}$ \\
\hline $\begin{array}{r}2 \\
12\end{array}$ & 1.38 & $\left.\begin{array}{l}1.39 \\
1.44\end{array}\right\}$ & 1.42 & 1.40 & -2.82 \\
\hline $\left.\begin{array}{l}10 \\
23\end{array}\right\}$ & 1.27 & $\left.\begin{array}{l}1.31 \\
1.47\end{array}\right\}$ & 1.39 & 1.33 & -8.63 \\
\hline $\left.\begin{array}{c}153 \\
189\end{array}\right\}$ & 1.45 & $\left.\begin{array}{l}1.36 \\
1.37\end{array}\right\}$ & 1.36 & 1.40 & +6.62 \\
\hline $\left.\begin{array}{c}313 \\
323\end{array}\right\}$ & 1.49 & $\left.\begin{array}{l}1.51 \\
1.53\end{array}\right\}$ & 1.52 & 1.50 & -1.97 \\
\hline $\left.\begin{array}{c}302 \\
315\end{array}\right\}$ & 1.38 & $\left.\begin{array}{l}1.48 \\
1.47\end{array}\right\}$ & 1.48 & 1.43 & -6.76 \\
\hline $\left.\begin{array}{l}136 \\
172\end{array}\right\}$ & 1.48 & $\left.\begin{array}{l}1.45 \\
1.45\end{array}\right\}$ & 1.45 & 1.46 & +2.07 \\
\hline Averag & & & & 1.42 & $-1.9 \pm 5.6$ \\
\hline E1emen & average & & & $1.38(c)$ & -- \\
\hline
\end{tabular}

(a) Gamma scans of corner fuel stacks while in block (see Fig. 3-4).

(b) Gamma scans of individual fuel stacks after removal from element.

(c) Average radial power (relative to block average) was 1.027 for the 12 fuel stacks. Average burnup divided by this factor to obtain element average burnùp. 
TABLE $3-19$

COMPARISON OF CALCULATED AND MEASURED FUEL BURNUP FOR FSV FUEL ELEMENT 1-0743

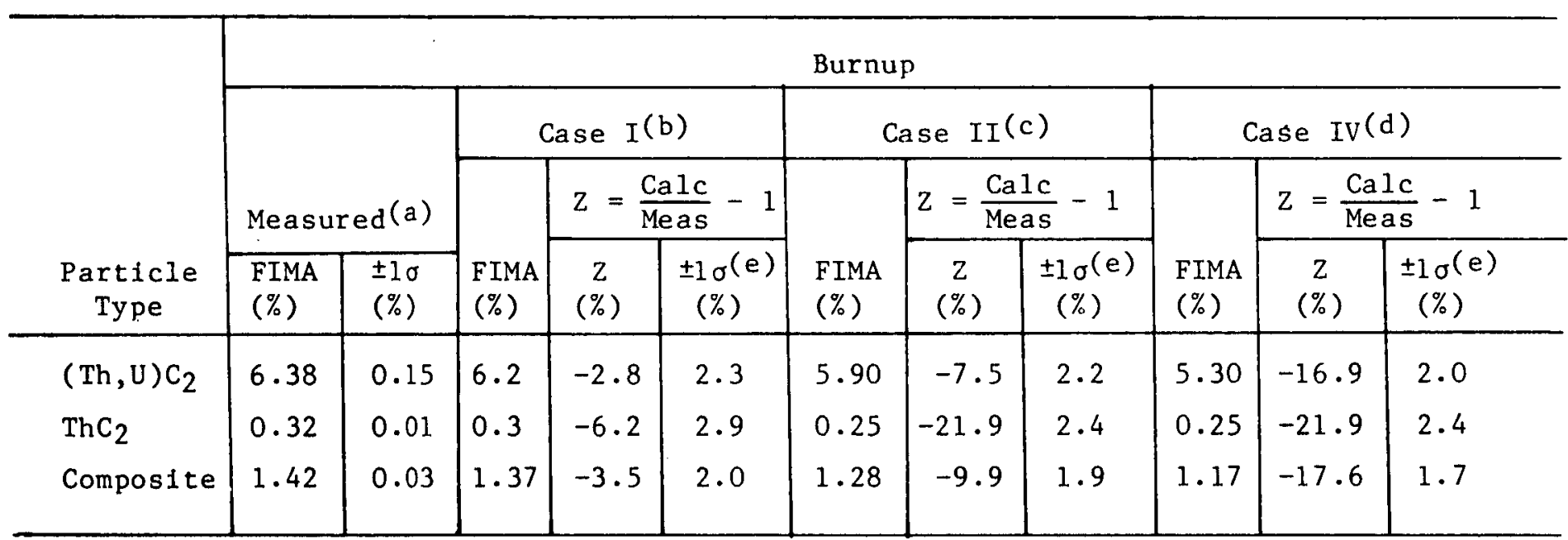

(a) Determined by averaging (Th,U) $C_{2}$ burnups at location of monitors 21 and 81 and $\mathrm{ThC}_{2}$ burnups for fuel rods $12-4$ and $279-3$. These averages should be approximately equivalent to element average burnups.
(b) SURVEY-detailed GAUGE analysis.
(c) GATT analysis.
(d) Calculations based on FEVER-calculated fluxes.
${ }^{(e)}$ Progressed uncertainty due to measurement uncertainty only. 
TABLE $3-20$

COMPARISON OF CALCULATED AND MEASURED URANIUM ISOTOPIC CONCENTRATIONS FOR UC 2 BURNUP MONITORS IRRADIATED IN FSV FUEL ELEMENT 1-0743

\begin{tabular}{|c|c|c|c|c|c|}
\hline \multirow[b]{4}{*}{ Isotope } & \multicolumn{5}{|c|}{ Isotopic Concentration } \\
\hline & \multirow{2}{*}{\multicolumn{2}{|c|}{ Measured (a) }} & \multirow{3}{*}{$\begin{array}{l}\text { Calculated (b) } \\
\text { Atom Percent }\end{array}$} & \multirow{2}{*}{\multicolumn{2}{|c|}{$\begin{array}{l}\text { Relative Difference } \\
\mathrm{Z}=\frac{\text { Calc }}{\text { Meas }}-1(\%)\end{array}$}} \\
\hline & & & & & \\
\hline & Atom Percent & $\pm \sigma$ & & Z & $\pm 1 \sigma(c)$ \\
\hline $\mathrm{U}-234$ & 0.797 & 0.002 & 0.8 & 0.38 & 0.25 \\
\hline $\mathrm{U}-235$ & 79.62 & 0.02 & 82.6 & 3.74 & 0.03 \\
\hline $\mathrm{U}-236$ & 10.98 & 0.02 & 8.9 & -18.94 & 0.15 \\
\hline $\mathrm{U}-238$ & 8.60 & 0.01 & 7.7 & -10.46 & 0.10 \\
\hline
\end{tabular}

(a) Average values for monitors 21 and 81 . The average neutron flux for these two monitors is approximately equivalent to the element average flux.

(b) Calculations based on fluxes obtained from the FEVER code. (c) Progressed uncertainty due to measurement uncertainty only. 


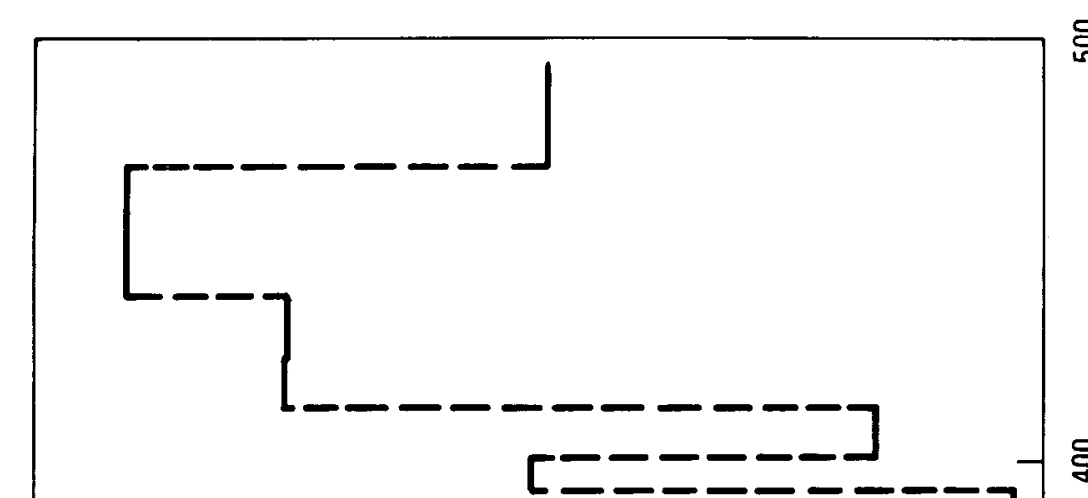

웅

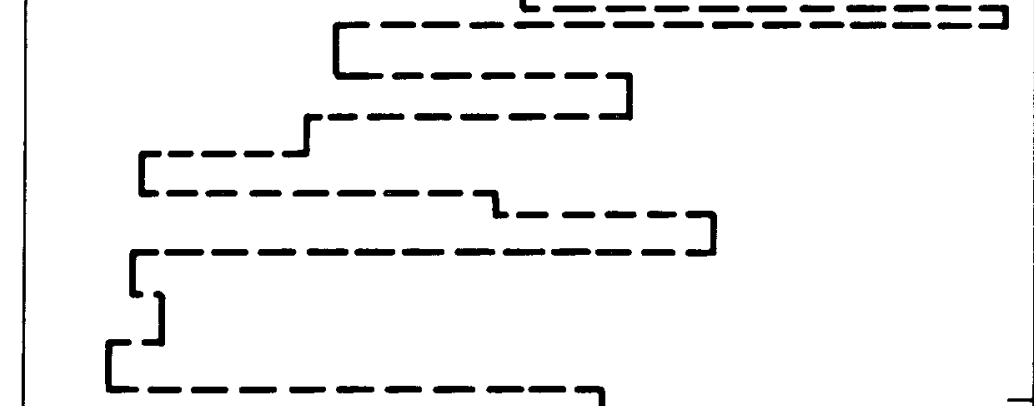

ఫ

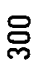

$-1$

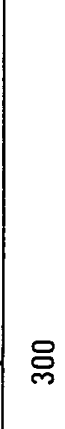

E- - - - - - -
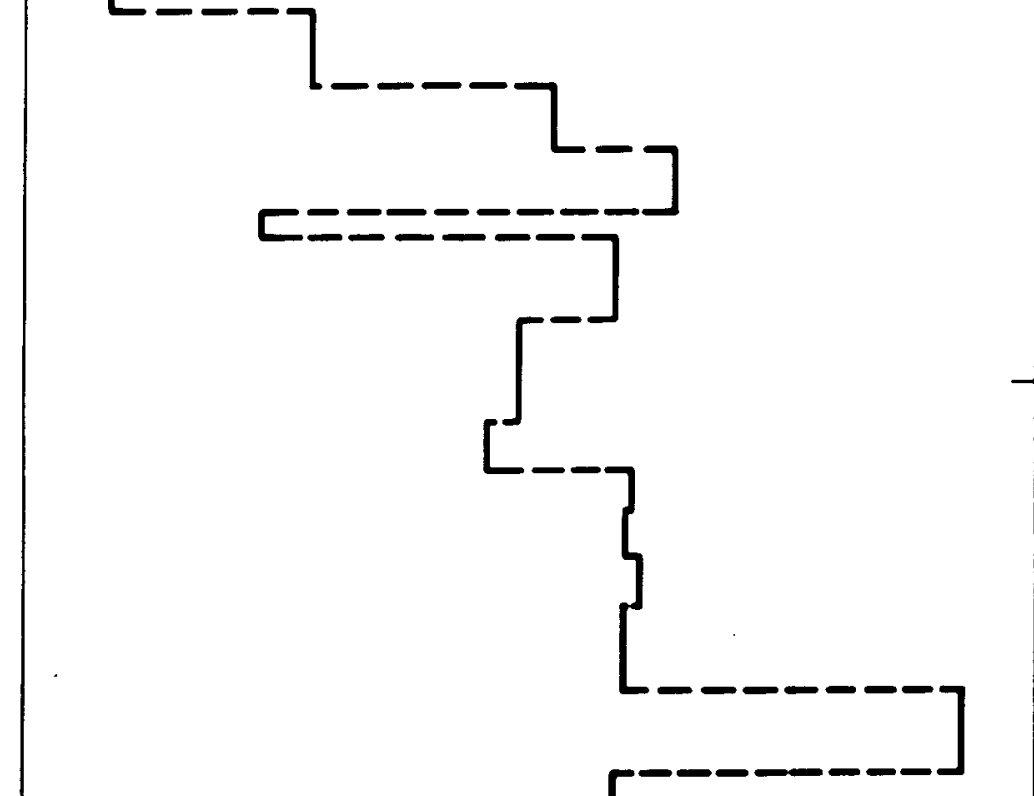

$\ominus$

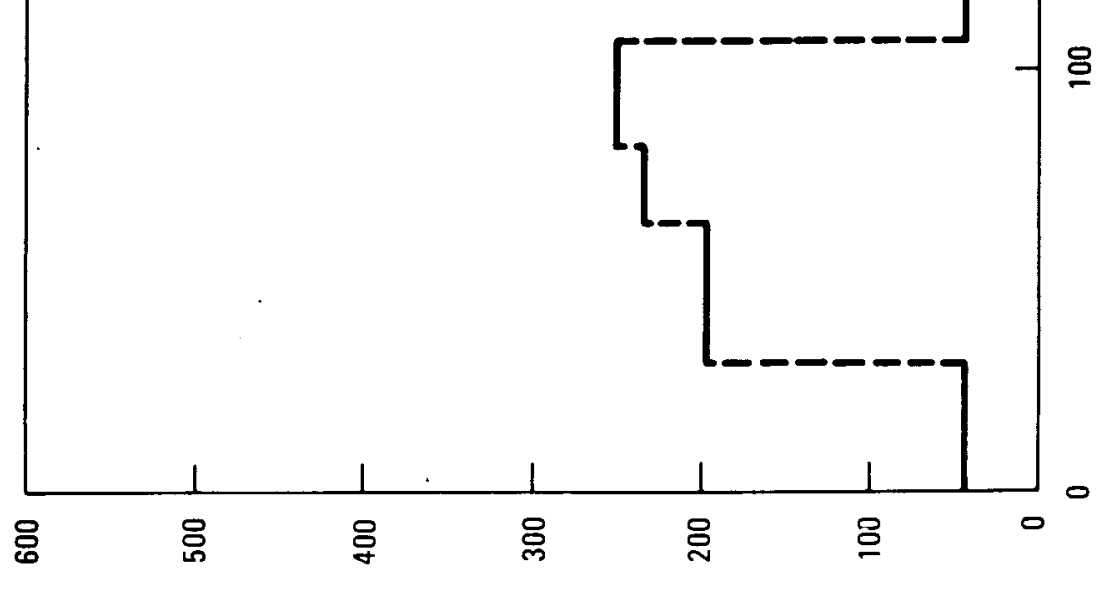

(MW) Y $\mathrm{MMOd} \exists \mathrm{YOO}$ 


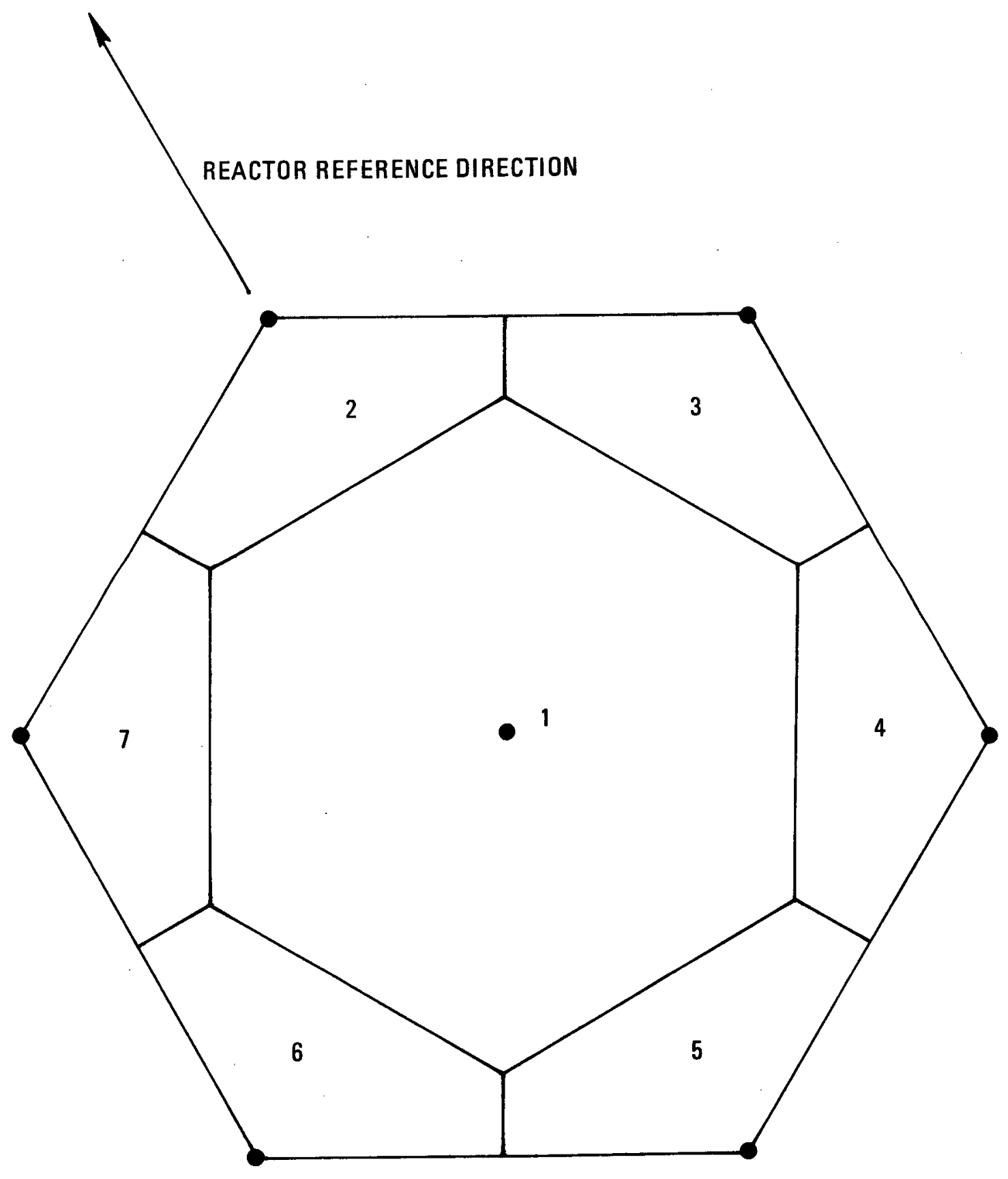

Fig. 3-2. Local point numbering for GAUGE-SURVEY analysis 


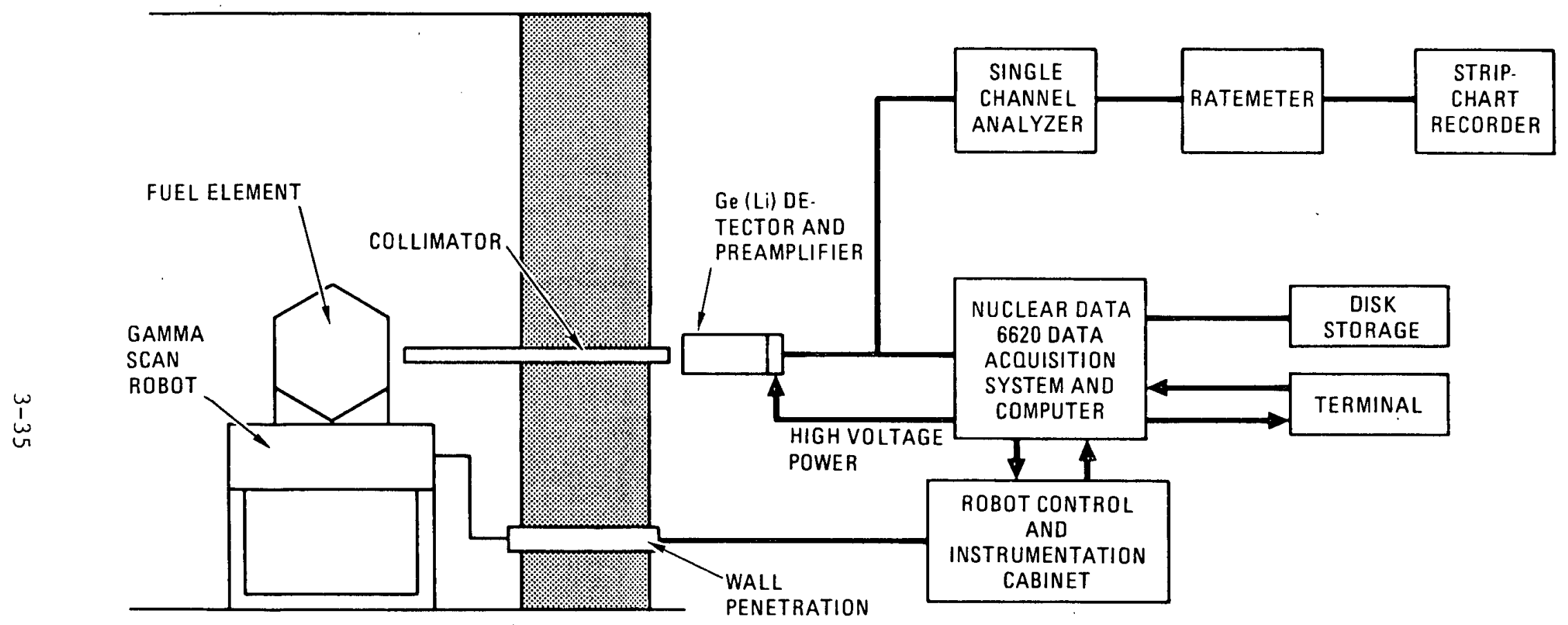

Fig. 3-3. System for scanning FSV core components 


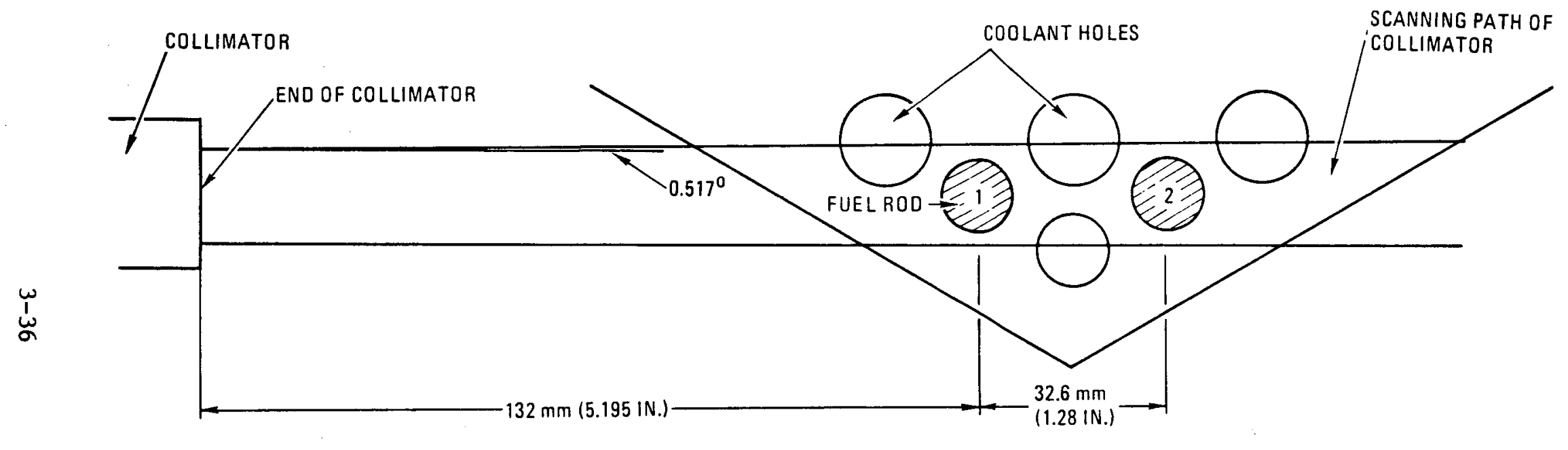

Fig. 3-4. Scanning geometry 1: axial corner scans 


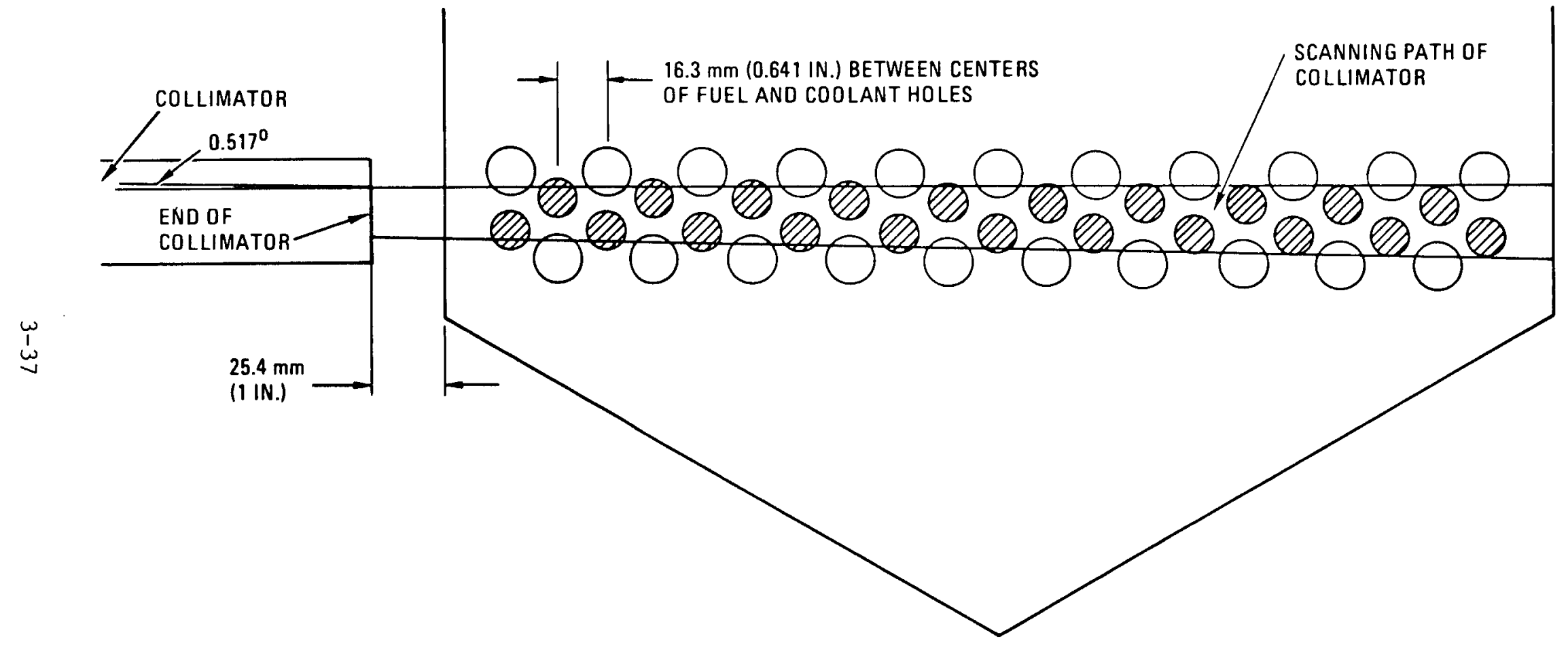

Fig. 3-5. Scanning geometry 2: axia1 side-face scans 


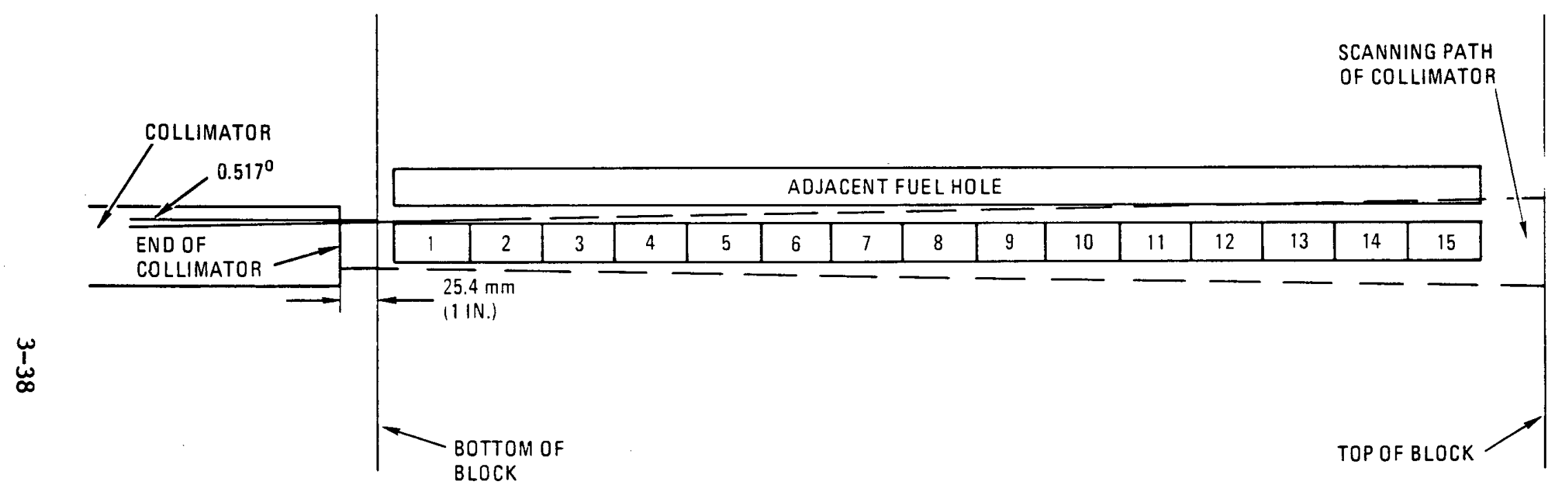

Fig. 3-6. Scanning geometry 3: end-on scans 


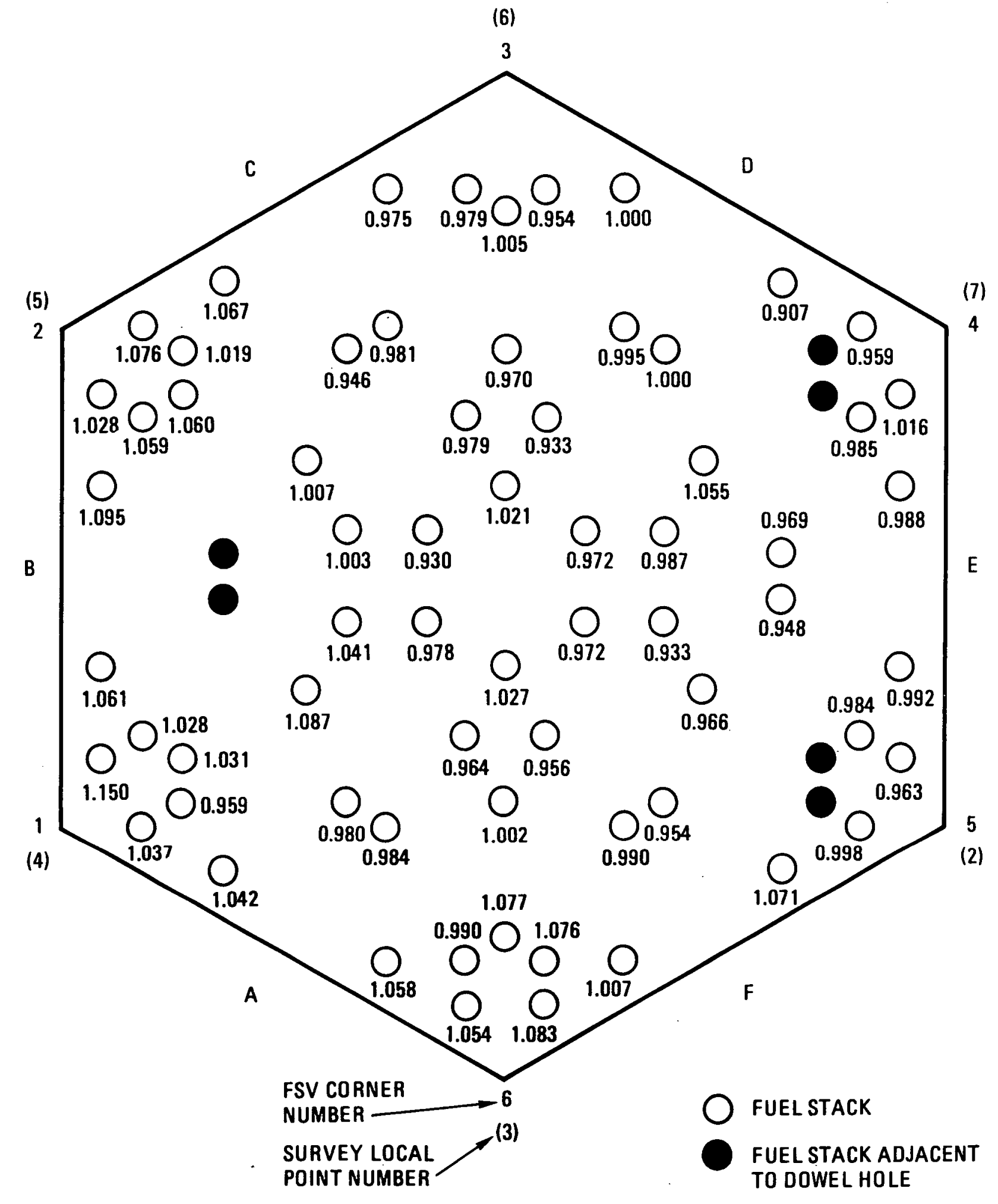

Fig. 3-7. Measured time-averaged radial power distribution for FSV fuel element 1-0743 (normalized Cs-137 distribution from end-on gamma scanning of fuel stacks at bottom of element) 


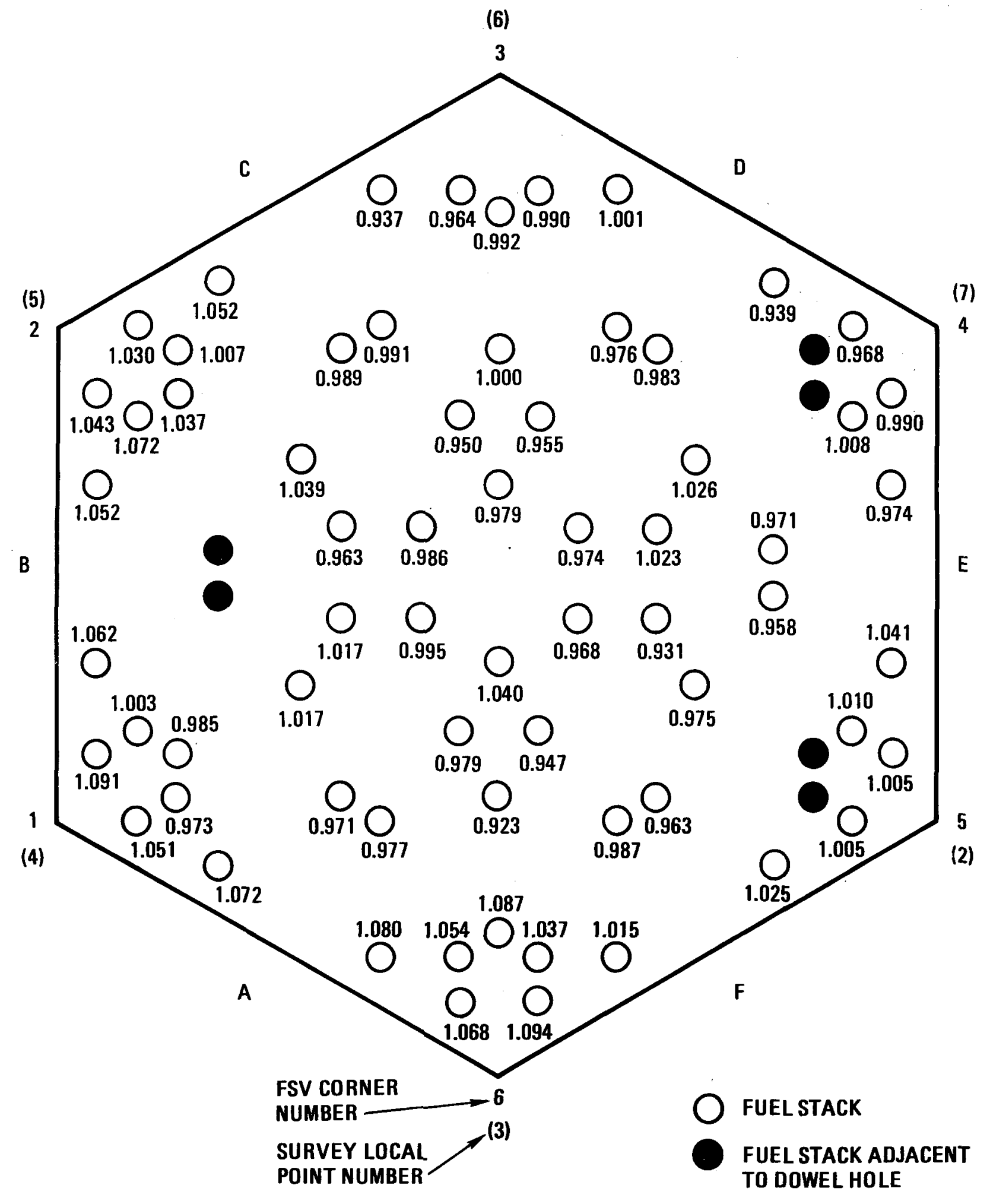

Fig. 3-8. Measured EOL distribution for FSV fuel element 1-0743 (normalized Zr-95 distribution obtained from end-on gamma scanning of fuel stacks at bottom of element) 


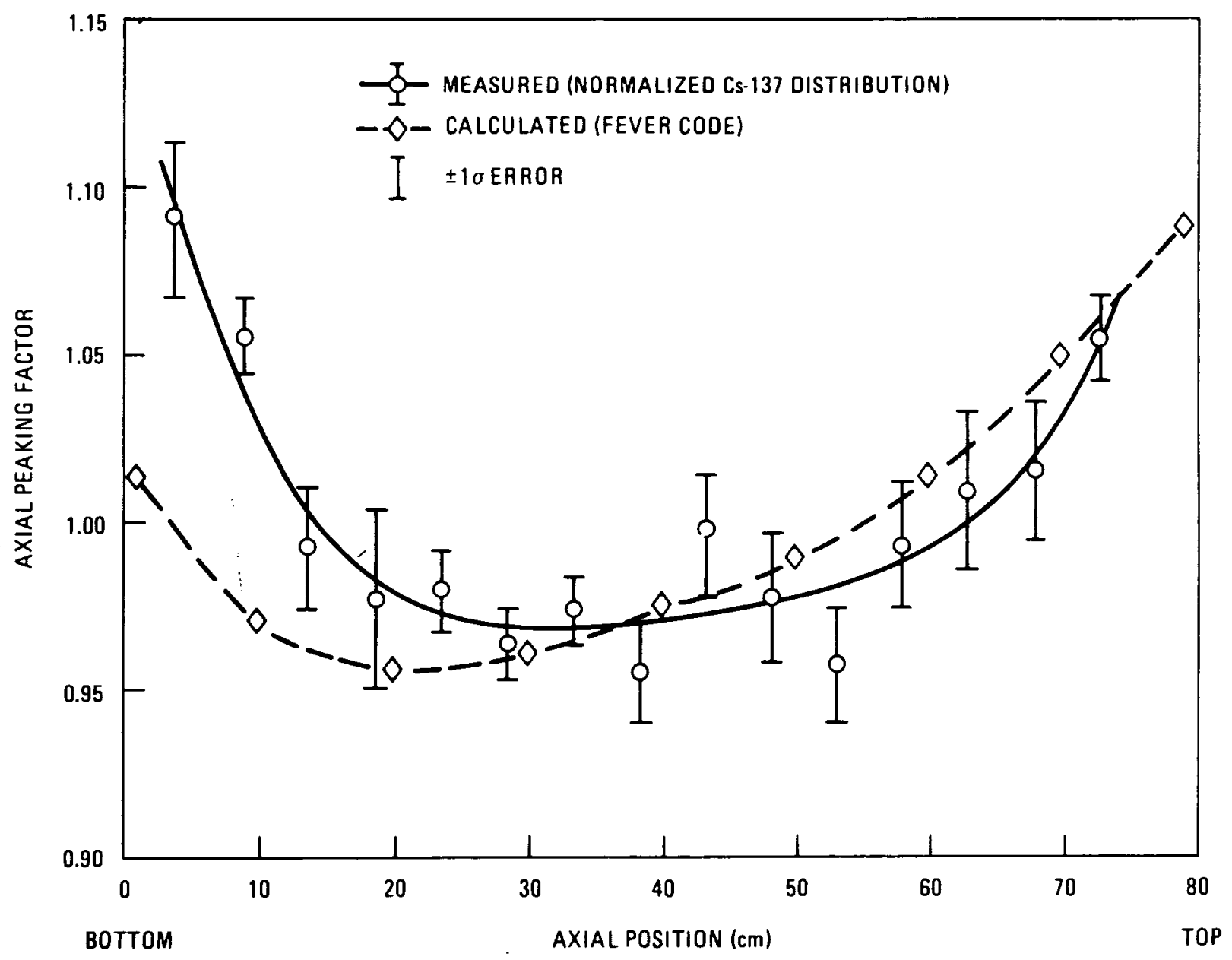

Fig. 3-9. Measured and calculated time-averaged axial power profiles for FSV fue1 element 1-0743 


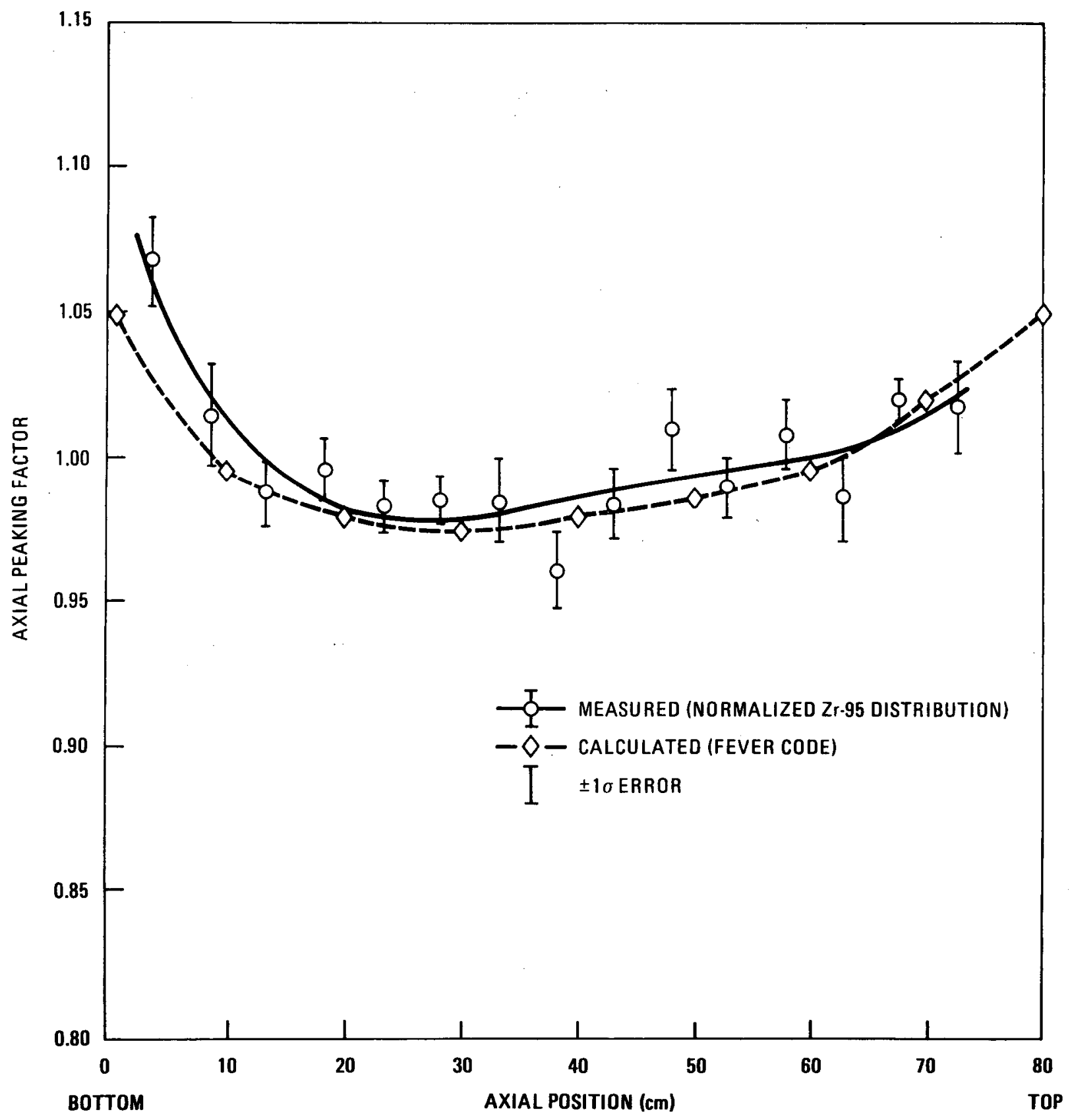

Fig. 3-10. Measured and calculated EOL axial power profiles for FSV fuel element 1-0743 


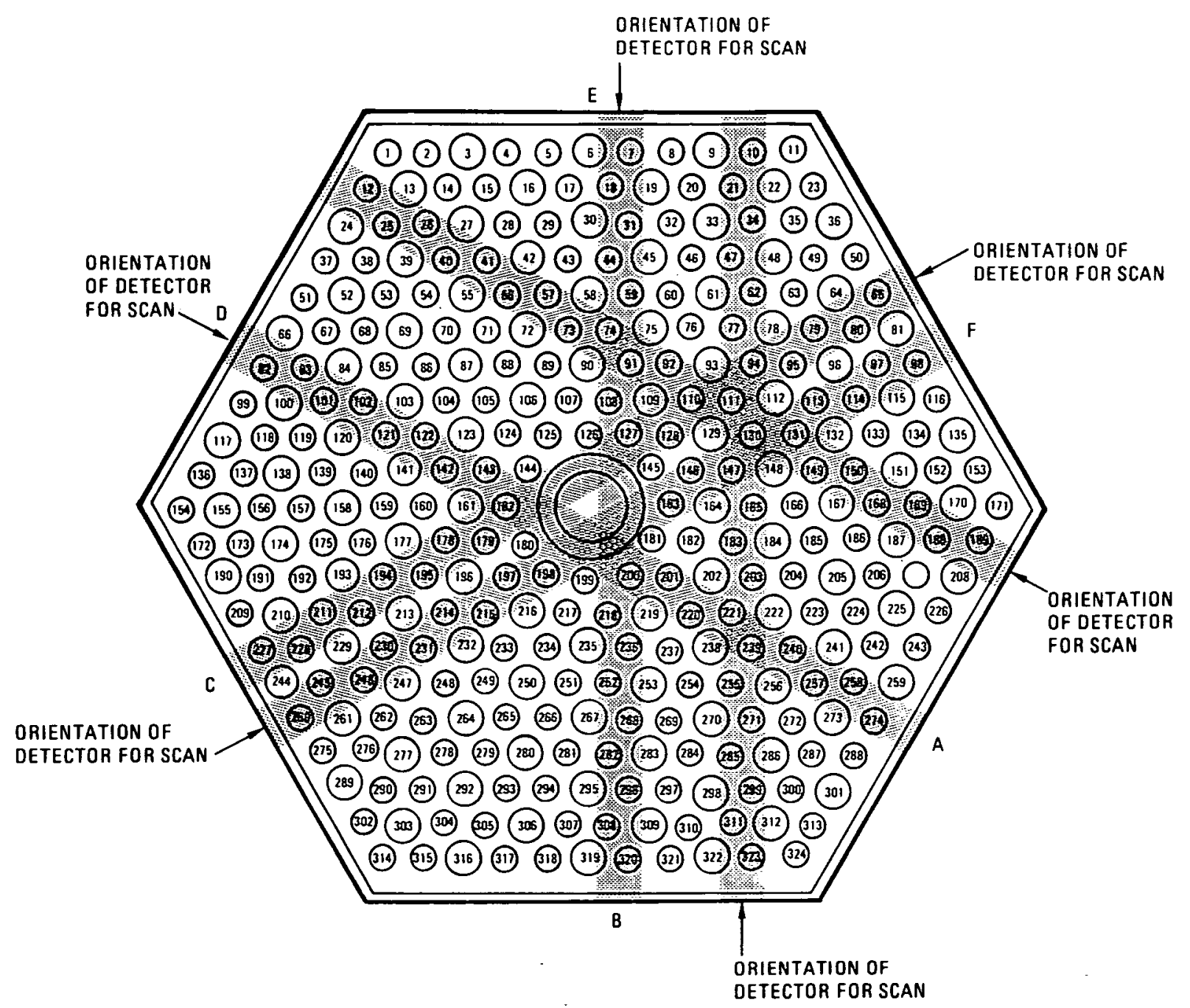

Fig. 3-11. Cross-sectional view showing portion of element observed by detector for six axial scans averaged to give measured power profiles 


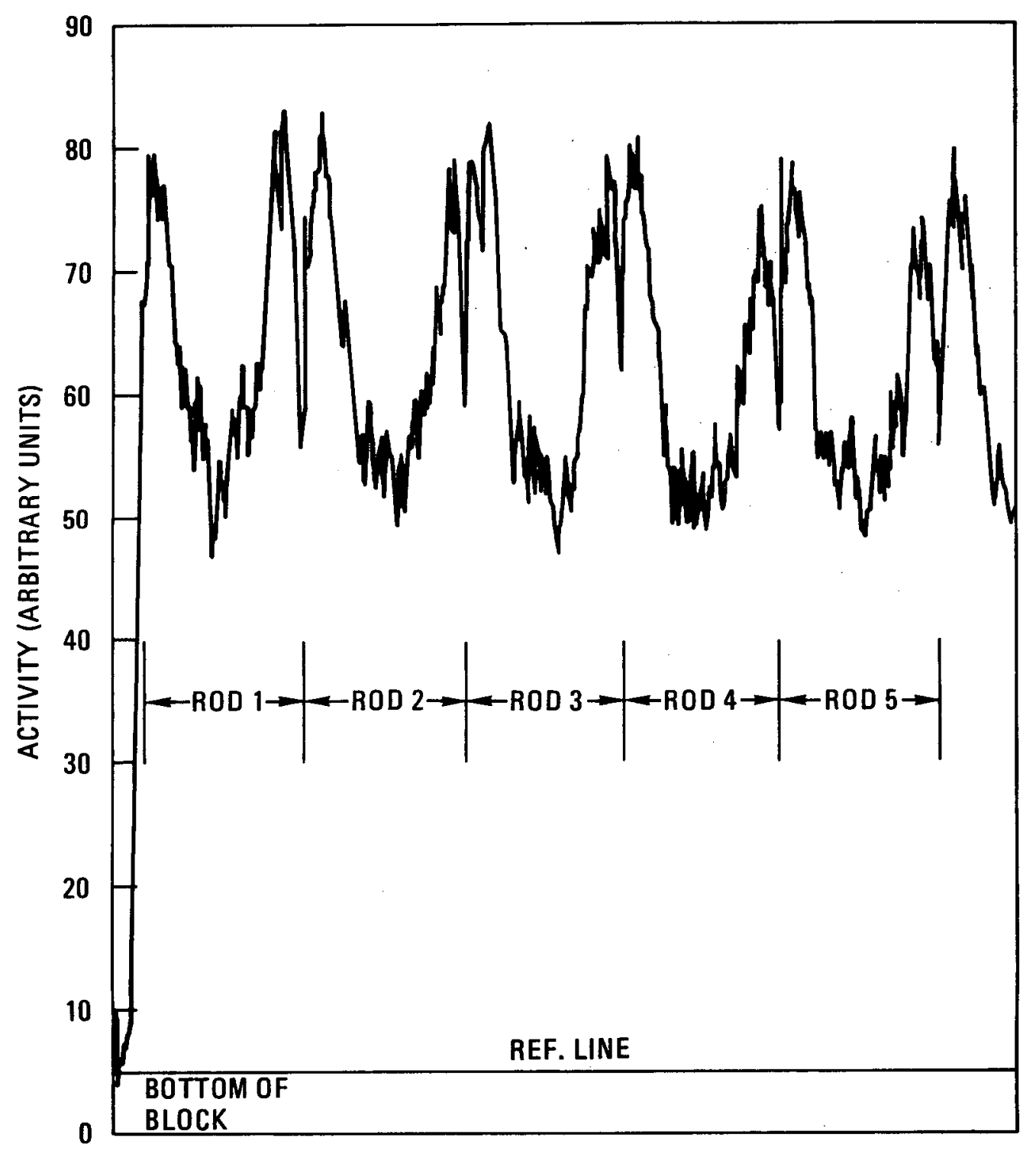

Fig. 3-12. Typical Cs-137 trace (partial) for axial scan of FSV fuel element 1-0743 


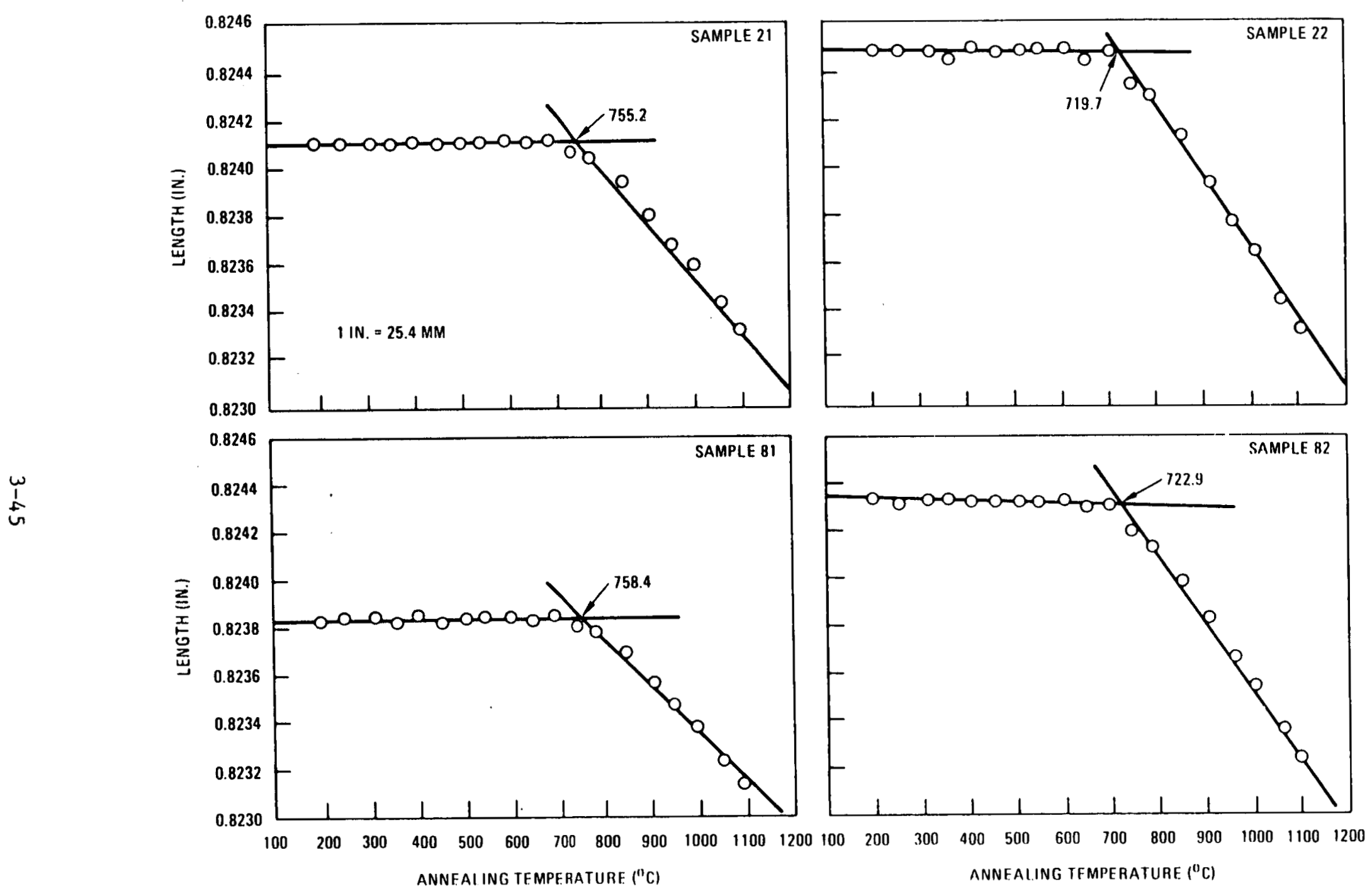

Fig. 3-13. Determination of irradiation temperatures for SiC pellets irradiated in FSV fuel element 1-0743 


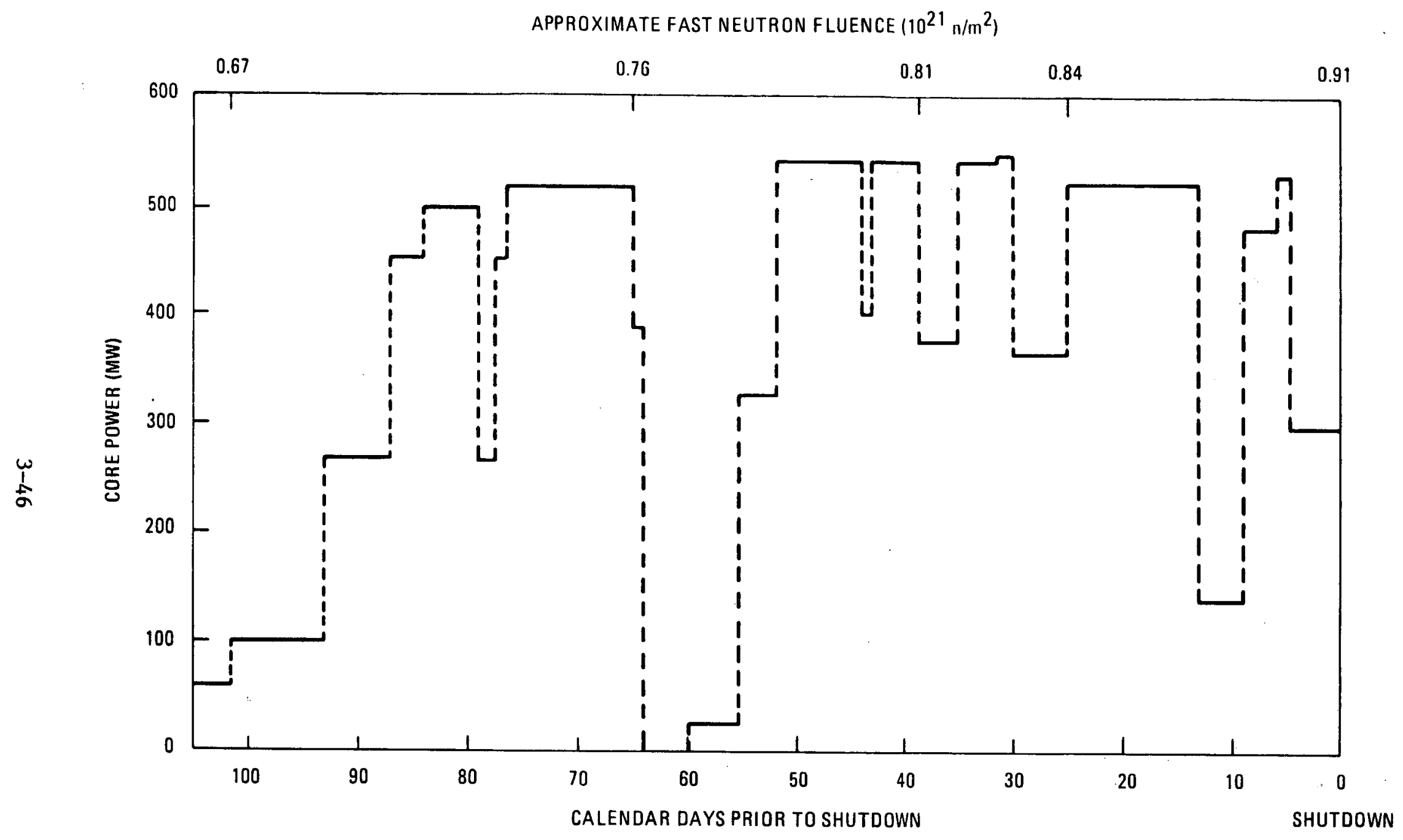

Fig. 3-14. Core power history over the last $\sim 2 \times 10^{24} \mathrm{n} / \mathrm{m}^{2}(\mathrm{E}>29 \mathrm{fJ})_{\text {HTGR }}$ exposure for FSV fuel element
$1-0743$ 


\section{RESULTS OF POSTIRRADIATION EXAMINATION}

\subsection{EXAMINATION OF GRAPHITE FUEL BLOCK}

\subsubsection{Visual Examination}

Like all of the segment 1 fuel elements examined in the hot service facility at FSV, fuel element 1-0743 was in good condition. No cracks were observed on any of the element surfaces. All observed abnormalities were surface markings only and had not etched the graphite to any harmful extent. Observed abnormalities included rub marks, soot deposits, scrapes, and scratches. Photographs of each side face are presented in Figs. 4-1 through 4-6, and the top surface is shown in Figs. 4-7 and 4-8. The bottom surface of the block was also photographed, but the quality of the pictures is too poor for them to be reproduced in this report. The element was visually examined again in the hot cell at GA, but nothing of significance was observed that had not been observed during the initial examination at FSV. The results of the visual examinations of all 51 segment 1 fuel and reflector blocks inspected at FSV are presented in detail in Ref. 1.

\subsubsection{Metrological Examination}

To verify the results of the metrological inspections performed by the metrology robot on segment 1 fuel elements at FSV following the first reload (Ref. 1), the dimensional measurements performed on element 1-0743 were repeated at GA using conventional hot cell measuring techniques. These techniques are described in Ref. 15. The results of these measurements are presented below. 
4.1.2.1. Irradiation-Induced Dimensional Changes. The metrology robot measurements and hot cell measurements for element 1-0743 are presented and compared with each other and with preirradiation measurements in Tables 4-1 through 4-10. The following results are based on the measurements taken in the GA hot cell:

1. The block average axial strain was $-0.17 \%$, corresponding to a length reduction of $1.32 \mathrm{~mm}$. Maximum and minimum length reductions were $1.73 \mathrm{~mm}$ and $0.91 \mathrm{~mm}$ adjacent to face $B$ and face $E$, respectively.

2. Block average axial strains determined from preirradiation and postirradiation distances were $-0.21 \%,-0.18 \%$, and $-0.19 \%$ for dimensions $L, M$, and $N$, respectively (see Fig. 2-5). These strains are consistent with each other and with the axial strain determined from element length measurements, indicating the axial strain to be uniform over the length of the block.

3. The block average radial strain was $-0.13 \%$, corresponding to a shrinkage of $0.46 \mathrm{~mm}$ across flats. The radial strain was nearly uniform for all three pairs of parallel side faces. The radial strain obtained from coolant hole diameter measurements was much higher, $-0.38 \%$, but is suspect because of the very small dimensional changes involved. The radial strain deduced from changes in the distances between coolant holes was $-0.16 \%$.

4. Face $B$ of the element was observed to have undergone the greatest convex bow and $f$ ace $E$ the greatest concave bow. The maximum bow for side faces $B$ and $E$ was $0.28 \mathrm{~mm}$.

4.1.2.2. Verification of Metrology Robot Measurements. In addition to the comparison between metrology robot and hot cell measurements for element 1-0743, a comparison between metrology robot measurements and Quality Control (QC) measurements on a spare (calibration) fuel block was performed 
to quantify and verify the accuracy of the metrology robot. The details of both comparisons have already been presented in Ref. 1 and are therefore omitted in this report. However, a summary of the results is given below.

Accuracy and bias statements developed from these comparisons for the various types of robot measurements are summarized in Table 4-11. The accuracy of the metrology robot was determined to be $\pm 0.18 \mathrm{~mm}(0.007 \mathrm{in.}) 1 \sigma$, or better, for each type of robot measurement after corrections were applied for observed measurement biases. Measurement biases were determined to be $0.05 \mathrm{~mm}(0.002 \mathrm{in.})$ or less for all robot measurements except length measurements. The bias (Actual -Robot) in the length measurements is 0.18 to $0.28 \mathrm{~mm}(0.007$ to $0.011 \mathrm{in.})$. The cause of the bias is not currently known but will be identified and corrected prior to inspection of FSV core segment 2. The length measurements for segment 1 fuel elements were corrected to account for this bias.

The comparisons of metrology robot data with the corresponding hot cell and $Q C$ measurements also revealed two mechanical defects in the robot which slightly affect the quality of robot measurements. These defects are discussed in Ref. 1. The segment 1 data have been corrected accordingly, and steps have been taken to eliminate the defects.

4.1.2.3. Comparison of Calculated and Measured Strain and Bow. Calculated and measured irradiation-induced strains and bow for fuel element 1-0743 are presented in Table 4-12. Calculated strains and bow were obtained from SURVEY/STRESS and are based on irradiation conditions from SURVEY. The SURVEY analysis is in turn based on the detailed GAUGE analysis of FSV cycle 1. In the sense that both calculated and measured strains and bow are small, the calculations and measurements are in good agreement. However, some discrepancies are observed. In particular, the bow in the element and the variation in the axial strain are greater than expected. The reader is 
directed to Ref. 1 for a systematic comparison of measured and calculated strains and bow for all 49 fuel elements examined (including element 1-0743) from FSV core segment 1 .

\subsection{DISASSEMBLY OF ELEMENT}

The postirradiation examination of fuel element 1-0743 was unique in that it was the first destructive examination performed at GA on a fuel element having the large HTGR prismatic block design. As such, it required the development of new devices and techniques for handling and disassembling the element. These devices and techniques have been employed, for the most part, with very satisfactory results. The disassembly of the element is described below.

\subsubsection{Coring}

A coring tool was developed and used to core out the fuel hole plugs at the top of the element and the graphite containment at the bottom. The device is positioned and aligned using the coolant holes and has six stations for the cutter to permit the six fuel holes surrounding a given coolant hole to be cored without relocating the tool. The coring tool is shown in Figs. 4-9 through 4-11. The cutter can be driven either directly by a drill motor or by a conventional ac motor via a flexible shaft. For hole diameters of $12.7 \mathrm{~mm}$, a cutter with an inside diameter of $16.5 \mathrm{~mm}$ and an outside diameter of $18.67 \mathrm{~mm}$ is used. This allows for some misalignment of the device and prevents damage to the fuel. The cored sections remain in place until forcibly removed. For the element, depths of cut ranged from $7.62 \mathrm{~mm}$ at the top surface to $11.4 \mathrm{~mm}$ at the bottom. A 40.4-mm depth of cut was required for fuel stacks situated beneath dowels. Once the device was positioned, the coring operation required only about 1 min per fuel stack, except for the stacks beneath dowels. 


\subsubsection{Plenum Depth Measurements}

Once all fuel holes had been cored at both the top and bottom of the element, the cored sections at the top were removed for the six holes containing precharacterized fuel rods. The distance from the top surface to the top fuel rod in each stack was then measured using a depth gauge. These measurements are given in Table 4-13. The measurement technique is illustrated in Fig. 4-12. An approximate 2.5-mm increase in plenum depth was observed for all six fuel holes.

\subsubsection{Removal of Fuel Rods}

The fuel rods were removed from the element by breaking out the cored sections and pushing the fuel stacks into a dual-tube receiving trough. The fuel stacks were pushed out of the element using either a metal rod or a special device designed to measure the push-out force. The push-out device and receiving trough are shown in Figs. 4-13 and 4-14, respectively. When measuring push-out forces, two forces are generally recorded: (1) the initial force required to start the stack moving and (2) the sustaining force required to continue pushing the rods. The initial force is generally higher, since more fuel rods are resisting.

Since the dimensional changes in the fuel rods and fuel body were quite small, no fuel rod-fuel body interaction, and consequently low push-out forces, were expected. The push-out forces measured for fuel element 1-0743 are given in Table 4-13. As expected, the push-out forces were generally low. However, in a few cases, the push-out forces required were considerable (up to $10 \mathrm{~kg}$ ). These high push-out forces are believed to be the result of misalignment between the fuel hole and receiving trough and of graphite debris from the breaking-out operation which become wedged between the fuel rods and fuel hole surface. It is concluded that there was no appreciable fuel rod -fuel block interaction in fuel element 1-0743. 


\subsection{EXAMINATION OF FUEL RODS}

\section{3:1. Visual Examination}

Following fuel stack removal, the six precharacterized stacks were measured for length (Table 4-13), and the fuel rods were individually photographed using the hot cell Kollmorgan periscope system. For the photography, the rods were placed in a trough with mirrors on each side at an angle of 90 deg relative to each other. This arrangement permitted approximately $300 \mathrm{deg}$ of the surface of each fuel rod to be photographed. In addition, stereophotography was performed in the metallography cell for each of the rods selected for fission gas release measurements (Section 4.3.4).

In general, the appearance of the fuel rods was good, although considerable chipping at the ends of the rods (Fig. 4-15) and some surface debonding (Fig. 4-16) were observed. No more than 21 failed particles were observed on the surface of any of the rods (Table 4-14 and Fig. 4-17). Very little particulate debris was found during unloading.

About $3 \%$ of the 3130 rods removed from the element were broken. Approximately $2 \%$ of these are thought to have been broken when pushed out of the block; the remaining $1 \%$ were probably broken prior to assembly of the element. Evidence of breakage prior to assembly was apparent in many instances. The orientation of the pieces in some of the broken rods was reversed so that one or both end caps were toward the middle of the rod rather than at the ends. Some broken fuel rods consisted of nonmatching pieces so that the composite length differed significantly from that of an unbroken rod. Also, some fuel stacks had broken pieces at each end with 14 unbroken rods in between. 


\subsubsection{Fuel Rod Metrology}

A representative sampling of fuel rods, including 70 of the 87 rods dimensionally characterized prior to irradiation (the other 17 were broken during unloading), was measured using an automated fuel rod measuring device. This device consists primarily of a slide with three linear potentiometers that engage the fuel rod and measure the diameter at three axial locations, a slide with one potentiometer for measuring the length, and a motor-driven support roller that holds and rotates the fuel rod. The quick action of the solenoids is dampened by small cylindrical shock absorbers working on the compression and vacuum of air. Several limit switches are attached for remotely signaling the computer that the slides are properly located for each measurement. This device is shown in Fig. 4-18 and an operational description is given in Ref. 16. The device is capable of making eight measurements per fuel rod in a few seconds. The time required to measure a stack of 15 fuel rods averaged about 22 min. (including fuel rod handling time), i.e., l-1/2 min per rod. When compared with the 6 min per rod required by the measuring technique employed for Peach Bottom fuel rods, it is evident that the automated fuel rod metrology device represents a major improvement in fuel rod measuring techniques.

The irradiation-induced strains* in the all-TRISO-particle fuel rods were found to be small and somewhat anisotropic, with the axial strain exceeding the radial. The average radial and axial strains for the 71 precharacterized fuel rods are $-0.36 \%$ and $-0.49 \%, r \in$ spectively. The stackaveraged fuel rod strains for each of the five fuel stacks containing

\footnotetext{
*The strain is calculated using the equation $\varepsilon=\mathrm{X}_{2} / \mathrm{X}_{1}-1$, where $\mathrm{X}_{2}$ is the postirradiation dimension and $x_{1}$ the preirradiation dimension. In calculating radial strain, the preirradiation dimensions measured using an air gauge were increased by $0.036 \mathrm{~mm}$ (Ref. 17) to make them compatible with the postirradiation micrometer-like measurements.
} 
precharacterized rods (all rods in the sixth stack were broken during unloading) are given in Table 4-15 and compared with predicted fuel rod strain curves in Fig. 4-19. The predicted strain curves were obtained using the model presented in Ref. 18 for irradiation-induced dimensional changes in HTGR fuel rods. It is observed that the predicted strains are about three times the measured strains. In addition, radial strains are predicted to be greater than axial strains, but the opposite occurs. One possible explanation is that the model was developed primarily from design data in the fast fluence range 4 to $10 \times 10^{25} \mathrm{n} / \mathrm{m} 2$ ( $\mathrm{E}>29 \mathrm{fJ}$ ) HTGR and extrapolated to low fluence. The curve for OPyC densification versus fluence is very steep at low fluence but is unverified, since no low-fluence data are available. This is a potential source of the observed discrepancies.

The detailed strain data for the precharacterized fuel rods are given in Tables 4-16 through 4-20.

\subsubsection{Fue1 Rod Strength Measurements}

Strength testing was performed on 13 irradiated fuel rods from element 1-0743 and 10 unirradiated rods from the same rod lot (CR-18-10165-1). The rods were compressed using an Instron tensile/compression testing machine at a rate of $0.002 \mathrm{~mm} / \mathrm{s}(0.005 \mathrm{in} . / \mathrm{min})$. A typical trace showing applied force as a function of time (and fuel rod compression) is shown in Fig. 4-20. Table 4-21 presents the failure load at rupture for each irradiated and unirradiated fuel rod. The mean failure load at rupture was $541.8 \pm 16.4$ $(1 \sigma) \mathrm{N}(121.8 \pm 3.7 \mathrm{lb})$ for the irradiated rods and $470.6 \pm 13.0(1 \sigma) \mathrm{N}$ $(105.8 \pm 2.9 \mathrm{lb})$ for the unirradiated rods. The mean compressive stresses at rupture for the irradiated and unirradiated rods were 4.3 and $3.7 \mathrm{MPa}$, respectively. The data indicate a statistically significant increase of approximately $15 \%$ in the compressive strength of the fuel rods with irradiation. 
Although the mean failure load at rupture for the irradiated rods was $541.8 \mathrm{~N}$, evidence of damage to the rods was observed for applied forces as low as $275 \mathrm{~N}$. This indicates that the maximum force applied in pushing fuel rods out of an element during disassembly should be limited to approximately $220 \mathrm{~N}(50$ 1b).

\subsubsection{Fission Gas Release}

Fission gas release for fuel rods irradiated in fuel element 1-0743 was measured before and after irradiation via neutron activation of the rods in the GA TRIGA reactor facility. Preirradiation measurements yield the uranium contamination and as-manufactured failed fissile particles. Postirradiation measurements yield the heavy metal contamination, as-manufactured failed particles, and in-pile coating failure. The in-pile coating failure can be estimated from the preirradiation and postirradiation fission gas release measurements using the calculation outlined in Ref. 18. This calculation also requires information concerning thorium contamination, asmanufactured defective fertile particles, and the fraction of fissions occurring in the fissile and fertile fuel at EOL.

The results of the fission gas release measurements are given in Table 4-22. Postirradiation measurements on groups of 3 and 10 rods and on 4 individual rods were performed. The $\mathrm{Kr}-85 \mathrm{~m} \mathrm{R} / \mathrm{B}$ value obtained for the 17 rods was $1.0 \times 10^{-4}$ (weighted average). The preirradiation $\mathrm{Kr}-85 \mathrm{~m} \mathrm{R} / \mathrm{B}$ value was $1.3 \times 10^{-4}$. The difference between the preirradiation and postirradiation $R / B$ values is attributed to the uncertainty of the measurement, which is approximately a factor of $1.6(1 \sigma)$ for $\mathrm{Kr}-85 \mathrm{~m}$ (Ref. 19).

Both the fissile and fertile particles potentially contribute to the postirradiation fission gas release. At EOL, approximately $65 \%$ of the fissions were occurring in the fissile particles and $35 \%$ in the fertile particles. The fission gas release results indicate that there was no 
significant fuel failure during irradiation, since there was no increase in the fission gas release. This conclusion is supported by the results of metallography.

\subsubsection{Meta1lography}

Four irradiated fuel rods and one unirradiated rod from the same rod lot were subjected to metallographic examination. The four irradiated rods were among the 17 rods for which fission gas release measurements were performed. Each rod was mounted in resin, ground, and polished. Prior to examination, all polished sections were passivated with a 50/50 solution of $\mathrm{HNO}_{3}$ and $\mathrm{H}_{2} \mathrm{O}$ to decrease the rate of hydrolysis of the $\mathrm{ThC}_{2}$ kernels. The entire polished surface of each rod was examined.

4.3.5.1. Results of Metallographic Examination. The fuel rod matrix appeared to be in good condition. No cracking was observed except for minor cracking in the matrix end cap. The microstructure of the matrix prior to and after irradiation is shown in Fig. 4-21. The irradiated microstructure is similar to the microstructure observed for FSV fuel rods irradiated in capsule F-30 (Ref. 20). The matrix porosity, which is composed of voids $\geq 50$ $\mu \mathrm{m}$, was measured for the irradiated rods and averaged $26 \%$. The macropor- osity of the unirradiated rod was $19 \%$. Both values are within the range of macroporosities observed for fuel rods from capsule F-30. An example of a radial cross section showing the macroporosity in the matrix is shown in Fig. 4-22.

The results of the metallographic examination of the four irradiated fuel rods are presented in Tables 4-23 and 4-24. The irradiation performance of the fissile and fertile TRISO coated particles was satisfactory. The microstructures of the particle types before and after irradiation are shown in Fig. 4-23. The microstructures had not changed significantly 
after being exposed to a fast neutron fluence of $\sim 1 \times 10^{25} \mathrm{n} / \mathrm{m}^{2}$

(E > $29 \mathrm{fJ})_{\mathrm{HTGR}}$ and a time-averaged temperature of $\sim 700^{\circ} \mathrm{C}$. Approximately 1500 fissile and 925 fertile particles were examined in the four rods.

The OPyC coating failure was $0.5 \%$ and $1.1 \%$ for the $(\mathrm{Th}, \mathrm{U}) \mathrm{C}_{2}$ and $\mathrm{ThC}_{2}$ particles, the SiC coating failure was $0.7 \%$ and $0.5 \%$, and the total coating failure was $0.3 \%(0.1 \leq \mathrm{F} \% \leq 0.5 ; 95 \%$ confidence $)$ and $0.2 \%(0.0 \leq \mathrm{F} \% \leq 0.7$; 95\% confidence). The coating failures were apparently as-manufactured failures which occurred during coating or fuel rod fabrication. The following evidence supports this conclusion:

1. The appearance of the failed particles. Two examples of failed particles are shown in Fig. 4-24. Particle (a) has the appearance of having been crushed, and part of the coating is missing in particle (b). In both cases, as-manufactured failure, rather than in-pile failure, is indicated.

2. The kernels of most particles with total coating failure were at least partially leached. This indicates as-manufactured failure, since the as-manufactured fuel rods were leached with $\mathrm{HCl}$.

3. The defective SiC coating fractions measured prior to irradiation using a burn-leach technique are the same as those measured for the four irradiated rods: $0.7 \%$ for $(\mathrm{Th}, \mathrm{U}) \mathrm{C}_{2}$ particles and $0.5 \%$ for $\mathrm{ThC}_{2}$ particles.

The chemical behavior of the TRISO particles was acceptable. No attack of the SiC coating was observed, and kernel migration was not seen. A small amount of a dense phase was observed in the buffer coating of some TRISO $(\mathrm{Th}, \mathrm{U}) \mathrm{C}_{2}$ particles. All the particles with this dense phase had a lowdensity, porous IPyC coating. The dense phase is attributed to fuel dispersion in as-manufactured fissile A particles (Refs. 21 and 22). The fuel dispersion was apparently caused by chlorine in the buffer coating. The chlorine had diffused through a permeable IPyC coating during the SiC 
coating operation. Fuel dispersion was observed in one out of 131 particles in the unirradiated rod. The fuel dispersion in an unirradiated and an irradiated particle is shown in Fig. 4-25. The fuel dispersion did not detrimentally affect the irradiation performance of the particles.

\subsubsection{Comparison of Calculated and Measured Fuel Failure. The meta1-} lographic examination of four irradiated fuel rods from fuel element 1-0743 revealed total coating failures of $0.3 \%$ and $0.2 \%$ for the $(\mathrm{Th}, \mathrm{U}) \mathrm{C}_{2}$ and $\mathrm{ThC}_{2}$ particles, respectively. However, based on the evidence discussed in Section 4.3.5.1, it was concluded that these were as-manufactured failures and that no in-pile failure occurred.

Fuel failure predictions for fuel element 1-0743 were obtained from SURVEY-PERFOR. In-pile failure due to manufacturing defects was predicted to be $0.32 \%$ for $(\mathrm{Th}, U) \mathrm{C}_{2}$ particles and $0.07 \%$ for $\mathrm{ThC}_{2}$ particles. No in-pile failure due to fission product-SiC interactions, kernel migration, or the pressure vessel failure mechanism was predicted for either particle. In view of the observation of no in-pile failure, the model for failure due to manufacturing defects appears to be conservative. 
TABLE $4-1$

FSV FUEL ELEMENT 1-0743 AXIAL DIMENSIONS

(inches) (a)

\begin{tabular}{|c|c|c|c|c|c|c|c|}
\hline $\begin{array}{l}\text { Corner } \\
\text { No. }\end{array}$ & Meas. & L Dim. (a) & $\ln (\mathrm{b})$ & $\operatorname{Dim}^{\mathrm{N}}(\mathrm{b})$ & ${ }_{\text {Dim. }}^{\mathrm{P}}(\mathrm{b})$ & $\lim ^{R}(b)$ & $\operatorname{Dim}^{\mathrm{S}}(\mathrm{b})$ \\
\hline 1 & $\begin{array}{l}\text { Pre I } \\
\text { Robot } \\
\text { PIE } \\
\text { Robot-Pre I } \\
\text { PIE-Pre I }\end{array}$ & $\begin{array}{l}9.0015 \\
8.975 \\
8.964 \\
-0.027 \\
-0.038\end{array}$ & $\begin{array}{r}9.003 \\
8.979 \\
8.976 \\
-0.024 \\
-0.027\end{array}$ & $\begin{array}{r}9.002 \\
8.981 \\
8.985 \\
-0.021 \\
-0.017\end{array}$ & $\begin{array}{r}2.251 \\
2.270 \\
2.2575 \\
+0.019 \\
+0.0065\end{array}$ & $\begin{array}{l}27.007 \\
26.935 \\
26.925 \\
-0.072 \\
-0.082\end{array}$ & $\begin{array}{l}31.2345 \\
31.150 \\
31.167 \\
-0.084 \\
-0.068\end{array}$ \\
\hline 2 & $\begin{array}{l}\text { Pre I } \\
\text { Robot } \\
\text { PIE } \\
\text { Robot-Pre I } \\
\text { PIE-Pre I }\end{array}$ & $\begin{array}{l}9.0015 \\
8.980 \\
8.987 \\
-0.022 \\
-0.015\end{array}$ & $\begin{array}{r}9.002 \\
8.970 \\
8.979 \\
-0.032 \\
-0.023\end{array}$ & $\begin{array}{r}9.003 \\
8.979 \\
8.980 \\
-0.024 \\
-0.023\end{array}$ & $\begin{array}{r}2.2515 \\
2.2610 \\
2.2575 \\
+0.010 \\
+0.006\end{array}$ & $\begin{array}{l}27.0065 \\
26.929 \\
26.946 \\
-0.078 \\
-0.061\end{array}$ & $\begin{array}{l}31.233 \\
31.147 \\
31.165 \\
-0.086 \\
-0.068\end{array}$ \\
\hline 3 & $\begin{array}{l}\text { Pre I } \\
\text { Robot } \\
\text { PIE } \\
\text { Robot-Pre I } \\
\text { PIE-Pre I }\end{array}$ & $\begin{array}{r}9.001 \\
8.987 \\
8.989 \\
-0.014 \\
-0.012\end{array}$ & $\begin{array}{r}9.002 \\
8.984 \\
8.984 \\
-0.018 \\
-0.018\end{array}$ & $\begin{array}{l}9.0015 \\
8.973 \\
8.982 \\
-0.029 \\
-0.020\end{array}$ & $\begin{aligned} & 2.2485 \\
& 2.273 \\
& 2.2525 \\
&+0.025 \\
&+0.004\end{aligned}$ & $\begin{array}{l}27.0045 \\
26.944 \\
26.955 \\
-0.061 \\
-0.050\end{array}$ & $\begin{array}{l}31.233 \\
31.159 \\
31.180 \\
-0.074 \\
-0.053\end{array}$ \\
\hline 4 & $\begin{array}{l}\text { Pre I } \\
\text { Robot } \\
\text { PIE } \\
\text { Robot-Pre I } \\
\text { PIE-Pre I }\end{array}$ & $\begin{array}{l}9.0025 \\
8.996 \\
8.991 \\
-0.007 \\
-0.012\end{array}$ & $\begin{array}{r}9.0005 \\
8.990 \\
8.996 \\
-0.011 \\
-0.005\end{array}$ & $\begin{array}{l}7.0025 \\
8.993 \\
8.986 \\
-0.010 \\
-0.017\end{array}$ & $\begin{array}{r}2.2515 \\
2.260 \\
2.2565 \\
+0.009 \\
+0.005\end{array}$ & $\begin{array}{l}27.0055 \\
26.979 \\
26.973 \\
-0.027 \\
-0.033\end{array}$ & $\begin{array}{l}31.232 \\
31.182 \\
31.195 \\
-0.050 \\
-0.037\end{array}$ \\
\hline 5 & $\begin{array}{l}\text { Pre I } \\
\text { Robot } \\
\text { PIE } \\
\text { Robot-Pre I } \\
\text { PIE-Pre I }\end{array}$ & $\begin{array}{r}9.002 \\
8.993 \\
8.993 \\
-0.009 \\
-0.009\end{array}$ & $\begin{array}{r}9.001 \\
8.990 \\
8.994 \\
-0.011 \\
-0.007\end{array}$ & $\begin{array}{l}9.0025 \\
8.994 \\
8.991 \\
-0.009 \\
-0.012\end{array}$ & $\begin{array}{c}2.253 \\
2.261 \\
2.2525 \\
+0.008 \\
-0.0005\end{array}$ & $\begin{array}{l}27.0055 \\
26.977 \\
26.978 \\
-0.029 \\
-0.028\end{array}$ & $\begin{array}{l}31.2315 \\
31.182 \\
31.196 \\
-0.050 \\
-0.036\end{array}$ \\
\hline 6 & $\begin{array}{l}\text { Pre I } \\
\text { Robot } \\
\text { PIE } \\
\text { Robot-Pre I } \\
\text { PIE-Pre.I }\end{array}$ & $\begin{array}{l}9.0015 \\
8.992 \\
8.977 \\
-0.010 \\
-0.025\end{array}$ & $\begin{array}{l}9.0025 \\
8.975 \\
8.986 \\
-0.028 \\
-0.017\end{array}$ & $\begin{array}{l}9.0025 \\
8.980 \\
8.988 \\
-0.023 \\
-0.015\end{array}$ & $\begin{aligned} & 2.2505 \\
& 2.278 \\
& 2.2525 \\
&+0.028 \\
&+0.002\end{aligned}$ & $\begin{array}{l}27.0065 \\
26.947 \\
26.951 \\
-0.060 \\
-0.056\end{array}$ & $\begin{array}{l}31.233 \\
31.164 \\
31.180 \\
-0.069 \\
-0.053\end{array}$ \\
\hline Robot & $\begin{array}{l}\text { Mean } \\
\text { Std Dev. }\end{array}$ & $\begin{array}{l}8.9872 \\
0.0082\end{array}$ & $\begin{array}{l}8.9813 \\
0.0081\end{array}$ & $\begin{array}{l}8.9833 \\
0.0084\end{array}$ & $\begin{array}{l}2.2672 \\
0.0076\end{array}$ & $\begin{array}{r}26.9518 \\
0.0213\end{array}$ & $\begin{array}{r}31.1640 \\
0.0152\end{array}$ \\
\hline PIE & $\begin{array}{l}\text { Mean } \\
\text { Std Dev. }\end{array}$ & $\begin{array}{l}8.9835 \\
0.0111\end{array}$ & $\begin{array}{l}8.9858 \\
0.0080\end{array}$ & $\begin{array}{l}8.9853 \\
0.0040\end{array}$ & $\begin{array}{l}2.2548 \\
0.0026\end{array}$ & $\begin{array}{r}26.9547 \\
0.0192\end{array}$ & $\begin{array}{r}31.1805 \\
0.0132\end{array}$ \\
\hline Pre I & $\begin{array}{l}\text { Mean } \\
\text { Std Dev. }\end{array}$ & $\begin{array}{l}9.0017 \\
0.0005\end{array}$ & $\begin{array}{l}9.0018 \\
0.0009\end{array}$ & $\begin{array}{l}9.0023 \\
0.0005\end{array}$ & $\begin{array}{l}2.2510 \\
0.0015\end{array}$ & $\begin{array}{r}27.0059 \\
0.0006\end{array}$ & $\begin{array}{r}31.2328 \\
0.0010\end{array}$ \\
\hline PIE-Pre & $\begin{array}{l}\text { I Mean } \\
\text { Std Dev. }\end{array}$ & $\begin{array}{r}-0.0185 \\
0.0110\end{array}$ & $\begin{array}{r}-0.0162 \\
0.0087\end{array}$ & $\begin{array}{r}-0.0173 \\
0.0038\end{array}$ & $\begin{array}{r}+0.0038 \\
0.0027\end{array}$ & $\begin{array}{r}-0.0517 \\
0.0197\end{array}$ & $\begin{array}{r}-0.0525 \\
0.0141\end{array}$ \\
\hline$\frac{\text { PIE-Pre }}{\text { Pre I }}$ & I $(\%$ strain $)$ & -0.21 & -0.18 & -0.19 & +0.17 & -0.19 & -0.17 \\
\hline
\end{tabular}

(a) 1 in. $=25.4 \mathrm{~mm}$.

(b) See Fig. 2-5. 
TABLE 4-2

FSV FUEL ELEMENT 1-0743 TRANSVERSE DIMENSIONAL

CHANGE - MINIMUM DISTANCE BETWEEN COOLANT HOLES

(inches) (a)

\begin{tabular}{|c|c|c|c|c|c|c|c|c|c|c|c|}
\hline $\begin{array}{l}\text { Holes } \\
312 \text { to } 13\end{array}$ & & $\begin{array}{c}312 \text { to } \\
270\end{array}$ & $\begin{array}{c}270 \text { to } \\
219\end{array}$ & $\begin{array}{c}219 \text { to } \\
106\end{array}$ & $\begin{array}{c}106 \text { to } \\
55\end{array}$ & $\begin{array}{c}55 \text { to } \\
13\end{array}$ & $\begin{array}{c}312 \text { to } \\
13\end{array}$ & & & Mean & Std Dev. \\
\hline $\begin{array}{l}\text { Top of } \\
\text { block }\end{array}$ & $\begin{array}{l}\text { Pre I } \\
\text { Robot } \\
\text { PIE } \\
\text { Robot-Pre I } \\
\text { PIE-Pre I }\end{array}$ & $\begin{array}{c}1.594 \\
1.593 \\
1.5913 \\
-0.001 \\
-0.0027\end{array}$ & $\begin{array}{c}1.597 \\
1.593 \\
1.5978 \\
-0.004 \\
+0.0008\end{array}$ & $\begin{array}{l}3.818 \\
3.814 \\
3.8132 \\
-0.004 \\
-0.0048\end{array}$ & $\begin{array}{l}1.595 \\
1.593 \\
1.5942 \\
-0.002 \\
-0.0008\end{array}$ & $\begin{array}{c}1.601 \\
1.594 \\
1.5945 \\
-0.007 \\
-0.0065\end{array}$ & $\begin{array}{l}12.695 \\
12.688 \\
12.6813 \\
-0.007 \\
-0.0137\end{array}$ & & & $\begin{array}{r}1.5968 \\
1.5933 \\
1.5945 \\
-0.0033 \\
-0.0023\end{array}$ & $\begin{array}{l}0.0031 \\
0.0005 \\
0.0027 \\
0.0026 \\
0.0031 .\end{array}$ \\
\hline $\begin{array}{l}\text { Bottom of } \\
\text { block }\end{array}$ & $\begin{array}{l}\text { Pre I } \\
\text { PIE } \\
\text { PIE-Pre I }\end{array}$ & $\begin{array}{r}1.598 \\
1.5918 \\
-0.0062\end{array}$ & $\begin{array}{r}1.594 \\
1.5970 \\
+0.0030\end{array}$ & $\begin{array}{r}3.816 \\
3.8189 \\
+0.0029\end{array}$ & $\begin{array}{c}1.601 \\
1.6018 \\
+0.0008\end{array}$ & $\begin{array}{c}1.598 \\
1.5955 \\
-0.0025\end{array}$ & $\begin{array}{l}12.700 \\
12.6978 \\
-0.0022\end{array}$ & & & $\begin{array}{r}1.5978 \\
1.5965 \\
-0.0012\end{array}$ & $\begin{array}{l}0.0029 \\
0.0041 \\
0.0040\end{array}$ \\
\hline $\begin{array}{l}\text { Holes } \\
319 \text { to } 6\end{array}$ & & $\begin{array}{c}319 \text { to } \\
295\end{array}$ & $\begin{array}{c}295 \text { to } \\
267\end{array}$ & $\begin{array}{c}267 \text { to } \\
235\end{array}$ & $\begin{array}{c}235 \text { to } \\
90\end{array}$ & $\begin{array}{l}90 \text { to } \\
58\end{array}$ & $\begin{array}{c}58 \text { to } \\
30\end{array}$ & $\begin{array}{c}30 \text { to } \\
6\end{array}$ & $\begin{array}{r}319 \\
6\end{array}$ & Mean & Std Dev. \\
\hline $\begin{array}{l}\text { Top of } \\
\text { block }\end{array}$ & $\begin{array}{l}\text { Pre I } \\
\text { Robot } \\
\text { PIE } \\
\text { Robot-Pre I } \\
\text { PIE-Pre I }\end{array}$ & $\begin{array}{l}0.655 \\
0.646 \\
0.6548 \\
-0.009 \\
-0.0002\end{array}$ & $\begin{array}{c}0.655 \\
0.665 \\
0.6603 \\
+0.010 \\
+0.0053\end{array}$ & $\begin{array}{l}0.657 \\
0.639 \\
0.6572 \\
-0.018 \\
+0.0002\end{array}$ & $\begin{array}{c}4.505 \\
4.514 \\
4.4982 \\
+0.007 \\
-0.0068\end{array}$ & $\begin{array}{c}0.658 \\
0.657 \\
0.6599 \\
-0.001 \\
+0.0019\end{array}$ & $\begin{array}{l}0.660 \\
0.648 \\
0.6547 \\
-0.012 \\
-0.0058\end{array}$ & $\begin{array}{l}0.658 \\
0.658 \\
0.6583 \\
0 \\
+0.0003\end{array}$ & $\begin{array}{l}12.191 \\
12.181 \\
12.1788 \\
-0.010 \\
-0.0122\end{array}$ & $\begin{array}{r}0.6572 \\
0.6522 \\
0.6575 \\
-0.0050 \\
+0.0003\end{array}$ & $\begin{array}{l}0.0019 \\
0.0095 \\
0.0024 \\
0.0100 \\
0.0036\end{array}$ \\
\hline $\begin{array}{l}\text { Bottom of } \\
\text { block }\end{array}$ & $\begin{array}{l}\text { Pre I } \\
\text { PIE } \\
\text { PIE-Pre I }\end{array}$ & $\begin{array}{r}0.655 \\
0.6564 \\
+0.0014\end{array}$ & $\begin{array}{r}0.654 \\
0.6612 \\
+0.0072\end{array}$ & $\begin{array}{c}0.655 \\
0.6558 \\
+0.0008\end{array}$ & $\begin{array}{c}4.508 \\
4.5089 \\
+0.0009\end{array}$ & $\begin{array}{r}0.656 \\
0.6564 \\
+0.0004\end{array}$ & $\begin{array}{c}0.657 \\
0.6505 \\
-0.0065\end{array}$ & $\begin{array}{r}0.662 \\
0.6638 \\
+0.0018\end{array}$ & $\begin{array}{l}12.196 \\
12.1924 \\
-0.0036\end{array}$ & $\begin{array}{r}0.6565 \\
0.6574 \\
+0.0009\end{array}$ & $\begin{array}{l}0.0029 \\
0.0046 \\
0.0044\end{array}$ \\
\hline $\begin{array}{l}\text { Holes } \\
303 \text { to } 22\end{array}$ & & $\begin{array}{c}303 \text { to } \\
264\end{array}$ & $\begin{array}{c}264 \text { to } \\
216\end{array}$ & $\begin{array}{c}216 \text { to } \\
109\end{array}$ & $\begin{array}{c}109 \text { to } \\
61\end{array}$ & $\begin{array}{c}61 \text { to } \\
22\end{array}$ & $\begin{array}{c}303 \text { to } \\
22\end{array}$ & & & Mean & Std Dev. \\
\hline $\begin{array}{l}\text { Top of } \\
\text { block }\end{array}$ & $\begin{array}{l}\text { Pre I } \\
\text { Robot } \\
\text { PIE } \\
\text { Robot-Pre I } \\
\text { PIE-Pre I }\end{array}$ & $\begin{array}{c}1.596 \\
1.589 \\
1.5946 \\
-0.007 \\
-0.0014\end{array}$ & $\begin{array}{c}1.597 \\
1.590 \\
1.5942 \\
-0.007 \\
-0.0028\end{array}$ & $\begin{array}{l}3.823 \\
3.819 \\
3.8144 \\
-0.004 \\
-0.0086\end{array}$ & $\begin{array}{c}1.598 \\
1.585 \\
1.5932 \\
-0.013 \\
-0.0048\end{array}$ & $\begin{array}{l}1.596 \\
1.586 \\
1.5936 \\
-0.010 \\
-0.0024\end{array}$ & $\begin{array}{l}12.696 \\
12.669 \\
12.6806 \\
-0.027 \\
-0.0154\end{array}$ & & & $\begin{array}{r}1.5968 \\
1.5875 \\
1.5939 \\
-0.0093 \\
-0.0029\end{array}$ & $\begin{array}{l}0.0010 \\
0.0024 \\
0.0006 \\
0.0029 \\
0.0014\end{array}$ \\
\hline $\begin{array}{l}\text { Bottom of } \\
\text { block }\end{array}$ & $\begin{array}{l}\text { Pre I } \\
\text { PIE } \\
\text { PIE-Pre I }\end{array}$ & $\begin{array}{r}1.598 \\
1.5975 \\
-0.0005\end{array}$ & $\begin{array}{r}1.598 \\
1.5989 \\
+0.0009\end{array}$ & $\begin{array}{r}3.823 \\
3.8236 \\
+0.0006\end{array}$ & $\begin{array}{r}1.596 \\
1.5978 \\
+0.0018\end{array}$ & $\begin{array}{c}1.601 \\
1.5934 \\
-0.0076\end{array}$ & $\begin{array}{l}12.705 \\
12.7045 \\
-0.0005\end{array}$ & & & $\begin{array}{r}1.5983 \\
1.5969 \\
-0.0014\end{array}$ & $\begin{array}{l}3.0021 \\
0.0024 \\
0.0043\end{array}$ \\
\hline $\begin{array}{c}\text { Holes } \\
170 \text { to } 155\end{array}$ & & $\begin{array}{c}170 \text { to } \\
167\end{array}$ & $\begin{array}{c}167 \text { to } \\
164\end{array}$ & $\begin{array}{c}164 \text { to } \\
161\end{array}$ & $\begin{array}{c}161 \text { to } \\
158\end{array}$ & $\begin{array}{c}158 \text { to } \\
155\end{array}$ & $\begin{array}{c}170 \text { to } \\
155\end{array}$ & & & Mean & Std Dev. \\
\hline $\begin{array}{l}\text { Top of } \\
\text { block }\end{array}$ & $\begin{array}{l}\text { Pre I } \\
\text { Robot } \\
\text { PIE } \\
\text { Robot-Pre I } \\
\text { PIE-Pre I }\end{array}$ & $\begin{array}{l}1.594 \\
1.585 \\
1.5931 \\
-0.009 \\
-0.0009\end{array}$ & $\begin{array}{l}1.596 \\
1.589 \\
1.5911 \\
-0.007 \\
-0.0049\end{array}$ & $\begin{array}{l}3.819 \\
3.826 \\
3.8166 \\
+0.007 \\
-0.0024\end{array}$ & $\begin{array}{c}1.595 \\
1.593 \\
1.5931 \\
-0.002 \\
-0.0019\end{array}$ & $\begin{array}{c}1.594 \\
1.590 \\
1.5930 \\
-0.004 \\
-0.0010\end{array}$ & $\begin{array}{l}12.690 \\
12.684 \\
12.6781 \\
-0.006 \\
-0.0119\end{array}$ & & & $\begin{array}{r}1.5948 \\
1.5893 \\
1.5926 \\
-0.0055 \\
-0.0022\end{array}$ & $\begin{array}{l}0.0010 \\
0.0033 \\
0.0010 \\
0.0031 \\
0.0019\end{array}$ \\
\hline $\begin{array}{l}\text { Bottom of } \\
\text { block }\end{array}$ & $\begin{array}{l}\text { Pre I } \\
\text { PIE } \\
\text { PIE-Pre I }\end{array}$ & $\begin{array}{r}1.594 \\
1.5935 \\
-0.0005\end{array}$ & $\begin{array}{r}1.596 \\
1.5961 \\
-0.0001\end{array}$ & $\begin{array}{r}3.817 \\
3.8168 \\
-0.0002\end{array}$ & $\begin{array}{c}1.597 \\
1.5964 \\
-0.0006\end{array}$ & $\begin{array}{r}1.596 \\
1.5947 \\
-0.0013\end{array}$ & $\begin{array}{l}12.692 \\
12.6895 \\
-0.0025\end{array}$ & & & $\begin{array}{r}1.5958 \\
1.5952 \\
-0.0006\end{array}$ & $\begin{array}{l}0.0013 \\
0.0013 \\
0.0006\end{array}$ \\
\hline Holes & & $\begin{array}{l}13 \text { to } \\
22\end{array}$ & $\begin{array}{c}22 \text { to } \\
170\end{array}$ & $\begin{array}{c}170 \text { to } \\
312\end{array}$ & $\begin{array}{c}312 \text { to } \\
303\end{array}$ & $\begin{array}{c}303 \text { to } \\
155\end{array}$ & $\begin{array}{c}155 \text { to } \\
13\end{array}$ & & & Mean & Std Dev. \\
\hline $\begin{array}{l}\text { Top of } \\
\text { block }\end{array}$ & $\begin{array}{l}\text { Pre I } \\
\text { Robot } \\
\text { PIE } \\
\text { Robot-Pre I } \\
\text { PIE-Pre I }\end{array}$ & $\begin{array}{c}6.037 \\
6.028 \\
6.0326 \\
-0.009 \\
-0.0044\end{array}$ & $\begin{array}{c}6.036 \\
6.026 \\
6.0221 \\
-0.010 \\
-0.0139\end{array}$ & $\begin{array}{l}6.034 \\
6.025 \\
6.0243 \\
-0.009 \\
-0.0097\end{array}$ & $\begin{array}{l}6.036 \\
6.024 \\
6.0283 \\
-0.012 \\
-0.0077\end{array}$ & $\begin{array}{l}6.035 \\
6.027 \\
6.0276 \\
-0.008 \\
-0.0074\end{array}$ & $\begin{array}{c}6.038 \\
6.025 \\
6.0313 \\
-0.013 \\
-0.0067\end{array}$ & & & $\begin{array}{r}6.0360 \\
6.0258 \\
6.0277 \\
-0.0102 \\
-0.0083\end{array}$ & $\begin{array}{l}0.0014 \\
0.0015 \\
0.0040 \\
0.0019 \\
0.0032\end{array}$ \\
\hline $\begin{array}{l}\text { Bottom of } \\
\text { block }\end{array}$ & $\begin{array}{l}\text { Pre I } \\
\text { PIE } \\
\text { PIE-Pre I }\end{array}$ & $\begin{array}{r}6.036 \\
6.0416 \\
+0.0056\end{array}$ & $\begin{array}{r}6.037 \\
6.0405 \\
+0.0035\end{array}$ & $\begin{array}{c}6.035 \\
6.0328 \\
-0.0022\end{array}$ & $\begin{array}{r}6.035 \\
6.0338 \\
-0.0012\end{array}$ & $\begin{array}{r}6.038 \\
6.0345 \\
-0.0035\end{array}$ & $\begin{array}{r}6.037 \\
6.0423 \\
+0.0053\end{array}$ & & & $\begin{array}{r}6.0363 \\
6.0376 \\
+0.0013\end{array}$ & $\begin{array}{l}0.0012 \\
0.0043 \\
0.0040\end{array}$ \\
\hline
\end{tabular}

(a) 1 in. $=25.4 \mathrm{~mm}$. 
TABLE 4-3

FSV FUEL ELEYENT 1-0743

SQUARENESS DATUM PLANES (inches) (a)

\begin{tabular}{|c|c|c|c|c|c|c|c|c|c|c|}
\hline \multirow[b]{2}{*}{ Face } & \multirow[b]{2}{*}{ Meas. } & \multicolumn{9}{|c|}{$\begin{array}{l}\text { Maximum Displacement from Squareness at Vertical } \\
\text { Incremental Distance up Length of Block(b) }\end{array}$} \\
\hline & & 1 & 2 & 3 & 4 & 5 & 6 & 7 & 8 & 9 \\
\hline A & $\begin{array}{l}\text { Pre I } \\
\text { Robot } \\
\text { PIE } \\
\text { Robot-Pre I } \\
\text { PIE-Pre I }\end{array}$ & $\begin{array}{l}-0.0005 \\
+0.0049 \\
+0.0021 \\
+0.0054 \\
+0.0026\end{array}$ & $\begin{array}{l}-0.00 \\
+0.0058 \\
+0.0037 \\
+0.0068 \\
+0.0047\end{array}$ & $\begin{array}{l}-0.002 \\
+0.0076 \\
+0.0049 \\
+0.0096 \\
+0.0069\end{array}$ & $\begin{array}{l}-0.002 \\
+0.0085 \\
+0.0056 \\
+0.0105 \\
+0.0076\end{array}$ & $\begin{array}{l}-0.001 \\
+0.0084 \\
+0.0048 \\
+0.0094 \\
+0.0058\end{array}$ & $\begin{array}{l}0.000 \\
+0.0073 \\
+0.0045 \\
+0.0073 \\
+0.0045\end{array}$ & $\begin{array}{l}-0.002 \\
+0.0051 \\
+0.0035 \\
+0.0071 \\
+0.0055\end{array}$ & $\begin{array}{l}-0.0015 \\
+0.0030 \\
+0.0020 \\
+0.0045 \\
+0.0035\end{array}$ & $\begin{array}{l}-0.0001 \\
-0.0001 \\
-0.0001 \\
0 \\
0\end{array}$ \\
\hline B & $\begin{array}{l}\text { Pre I } \\
\text { Robot } \\
\text { PIE } \\
\text { Robot-Pre I } \\
\text { PIE-Pre I }\end{array}$ & $\begin{array}{l}+0.001 \\
+0.0070 \\
+0.0015 \\
+0.0060 \\
+0.0005\end{array}$ & $\begin{array}{l}+0.0005 \\
+0.0110 \\
+0.0030 \\
+0.0105 \\
+0.0025\end{array}$ & $\begin{array}{l}0.000 \\
+0.0120 \\
+0.0046 \\
+0.0120 \\
+0.0046\end{array}$ & $\begin{array}{l}+0.001 \\
+0.0130 \\
+0.0061 \\
+0.0120 \\
+0.0051\end{array}$ & $\begin{array}{l}-0.0005 \\
+0.0120 \\
+0.0076 \\
+0.0125 \\
+0.0081\end{array}$ & $\begin{array}{l}0.000 \\
+0.0100 \\
+0.0086 \\
+0.0100 \\
+0.0086\end{array}$ & $\begin{array}{l}-0.001 \\
+0.0070 \\
+0.0063 \\
+0.0080 \\
+0.0073\end{array}$ & $\begin{array}{l}-0.001 \\
+0.0030 \\
+0.0030 \\
+0.0040 \\
+0.0040\end{array}$ & $\begin{array}{l}-0.001 \\
-0.001 \\
-0.0010 \\
0 \\
0\end{array}$ \\
\hline $\mathrm{C}$ & $\begin{array}{l}\text { Pre I } \\
\text { Robot } \\
\text { PIE } \\
\text { Robot-Pre I } \\
\text { PIE-Pre I }\end{array}$ & $\begin{array}{l}+0.0005 \\
+0.0043 \\
+0.0027 \\
+0.0038 \\
+0.0022\end{array}$ & $\begin{array}{l}+0.001 \\
-0.0076 \\
+0.0045 \\
+0.0066 \\
+0.0035\end{array}$ & $\begin{array}{l}+0.001 \\
+0.0088 \\
+0.0057 \\
+0.0078 \\
+0.0047\end{array}$ & $\begin{array}{l}+0.001 \\
+0.0101 \\
+0.0065 \\
+0.0091 \\
+0.0055\end{array}$ & $\begin{array}{l}+0.001 \\
+0.0104 \\
+0.0068 \\
+0.0094 \\
+0.0058\end{array}$ & $\begin{array}{l}+0.0015 \\
+0.0087 \\
+0.0063 \\
+0.0072 \\
+0.0048\end{array}$ & $\begin{array}{l}+0.001 \\
+0.0068 \\
+0.0050 \\
+0.0058 \\
+0.0040\end{array}$ & $\begin{array}{l}+0.0005 \\
+0.0052 \\
+0.0039 \\
+0.0047 \\
+0.0034\end{array}$ & $\begin{array}{c}+0.0015 \\
+0.0015 \\
+0.0015 \\
0 \\
0\end{array}$ \\
\hline $\mathrm{D}$ & $\begin{array}{l}\text { Pre I } \\
\text { Robot } \\
\text { PIE } \\
\text { Robot-Pre I } \\
\text { PIE-Pre I }\end{array}$ & $\begin{array}{l}+0.0005 \\
-0.0004 \\
-0.0010 \\
-0.0009 \\
-0.0015\end{array}$ & $\begin{array}{l}+0.0005 \\
-0.0008 \\
-0.0020 \\
-0.0013 \\
-0.0025\end{array}$ & $\begin{array}{l}+0.001 \\
+0.0008 \\
-0.0023 \\
-0.0002 \\
-0.0033\end{array}$ & $\begin{array}{l}+0.001 \\
+0.0014 \\
-0.0024 \\
+0.0004 \\
-0.0034\end{array}$ & $\begin{array}{l}+0.001 \\
\div 0.0021 \\
-0.0023 \\
+0.0011 \\
-0.0033\end{array}$ & $\begin{array}{l}+0.0001 \\
+0.0007 \\
-0.0012 \\
+0.0006 \\
-0.0013\end{array}$ & $\begin{array}{l}+0.0015 \\
+0.0013 \\
-0.0011 \\
-0.0002 \\
-0.0026\end{array}$ & $\begin{array}{l}+0.002 \\
+0.0009 \\
+0.0004 \\
-0.0011 \\
-0.0024\end{array}$ & $\begin{array}{c}+0.0025 \\
+0.0025 \\
+0.0025 \\
0 \\
0\end{array}$ \\
\hline$E$ & $\begin{array}{l}\text { Pre I } \\
\text { Robot } \\
\text { PIE } \\
\text { Robot-Pre I } \\
\text { PIE-Pre I }\end{array}$ & $\begin{array}{l}0.000 \\
-0.0021 \\
-0.0042 \\
-0.0021 \\
-0.0042\end{array}$ & $\begin{array}{l}+0.0005 \\
-0.0022 \\
-0.0056 \\
-0.0027 \\
-0.0061\end{array}$ & $\begin{array}{l}0.000 \\
-0.0043 \\
-0.0081 \\
-0.0043 \\
-0.0081\end{array}$ & $\begin{array}{l}+0.0005 \\
-0.0054 \\
-0.0089 \\
-0.0059 \\
-0.0094\end{array}$ & $\begin{array}{l}+0.001 \\
-0.0046 \\
-0.0087 \\
-0.0056 \\
-0.0097\end{array}$ & $\begin{array}{l}+0.001 \\
-0.0047 \\
-0.0078 \\
-0.0057 \\
-0.0088\end{array}$ & $\begin{array}{l}+0.001 \\
-0.0038 \\
-0.0054 \\
-0.0048 \\
-0.0064\end{array}$ & $\begin{array}{l}+0.0015 \\
-0.0009 \\
-0.0016 \\
-0.0024 \\
-0.0031\end{array}$ & $\begin{array}{c}+0.002 \\
+0.0020 \\
+0.0020 \\
0 \\
0\end{array}$ \\
\hline $\mathrm{F}$ & $\begin{array}{l}\text { Pre I } \\
\text { Robot } \\
\text { PIE } \\
\text { Robot-Pre I } \\
\text { PIE-Pre I } \\
\end{array}$ & $\begin{array}{l}0.000 \\
-0.0014 \\
-0.0020 \\
-0.0014 \\
-0.0020 \\
\end{array}$ & $\begin{array}{l}+0.0005 \\
-0.0028 \\
-0.0036 \\
-0.0033 \\
-0.0041 \\
\end{array}$ & $\begin{array}{l}+0.0005 \\
-0.0032 \\
-0.0047 \\
-0.0037 \\
-0.0052 \\
\end{array}$ & $\begin{array}{l}0.000 \\
-0.0026 \\
-0.0050 \\
-0.0026 \\
-0.0050 \\
\end{array}$ & $\begin{array}{l}0.000 \\
-0.0029 \\
-0.0051 \\
-0.0029 \\
-0.0051 \\
\end{array}$ & $\begin{array}{l}0.000 \\
-0.0043 \\
-0.0041 \\
-0.0043 \\
-0.0041 \\
\end{array}$ & $\begin{array}{l}+0.0005 \\
-0.0037 \\
-0.0035 \\
-0.0042 \\
-0.0040 \\
\end{array}$ & $\begin{array}{l}+0.0005 \\
-0.0041 \\
-0.0018 \\
-0.0046 \\
-0.0023 \\
\end{array}$ & $\begin{array}{c}+0.0005 \\
+0.0005 \\
+0.0005 \\
0 \\
0 \\
\end{array}$ \\
\hline Robot & $\begin{array}{l}\text { Mean } \\
\text { Std. Dev. }\end{array}$ & $\begin{array}{r}+0.0021 \\
0.0038\end{array}$ & $\begin{array}{r}+0.0031 \\
0.0058\end{array}$ & $\begin{array}{r}+0.0036 \\
0.0068\end{array}$ & $\begin{array}{r}+0.0042 \\
0.0074\end{array}$ & $\begin{array}{r}+0.0042 \\
0.0071\end{array}$ & $\begin{array}{r}+0.0030 \\
0.0066\end{array}$ & $\begin{array}{r}+0.0021 \\
0.0050\end{array}$ & $\begin{array}{r}+0.0012 \\
0.0033\end{array}$ & $\begin{array}{r}+0.0009 \\
0.0013\end{array}$ \\
\hline PIE & $\begin{array}{l}\text { Mean } \\
\text { Std. Dev. }\end{array}$ & $\begin{array}{r}-0.0002 \\
0.0027\end{array}$ & $\begin{array}{l}0 \\
0.0043\end{array}$ & $\begin{array}{l}0 \\
0.0058\end{array}$ & $\begin{array}{r}+0.0003 \\
0.0066\end{array}$ & $\begin{array}{r}+0.0005 \\
0.0068\end{array}$ & $\begin{array}{r}+0.0011 \\
0.0064\end{array}$ & $\begin{array}{r}+0.0008 \\
0.0048\end{array}$ & $\begin{array}{r}+0.0010 \\
0.0024\end{array}$ & $\begin{array}{r}+0.0009 \\
0.0013\end{array}$ \\
\hline
\end{tabular}

(a) 1 in. $=25.4 \mathrm{~mm}$.

(b) See detail $\mathrm{T}$ in Fig. 2-5 for interpretation of + and - values. 
TABLE 4-4

FSV FUEL ELEMENT 1-0743

COOLANT HOLE DIAIETERS

(inches) (a)

\begin{tabular}{|c|c|c|c|}
\hline \multirow{2}{*}{$\begin{array}{l}\text { Hole } \\
\text { No. }\end{array}$} & \multirow[b]{2}{*}{ Meas. } & \multicolumn{2}{|c|}{ Hole Diameter $(\mathrm{J})^{(\mathrm{b})}$} \\
\hline & & Top & Bottom \\
\hline 13 & $\begin{array}{l}\text { Pre I } \\
\text { Robot } \\
\text { PIE } \\
\text { Robot-Pre I } \\
\text { PIE-Pre I }\end{array}$ & $\begin{array}{l}0.625 \\
0.625 \\
0.6228 \\
0 \\
-0.0022\end{array}$ & $\begin{array}{l}0.625 \\
\mathrm{ND}(\mathrm{c}) \\
0.6234 \\
\mathrm{ND} \\
-0.0016\end{array}$ \\
\hline 22 & $\begin{array}{l}\text { Pre I } \\
\text { Robot } \\
\text { PIE } \\
\text { Robot-Pre I } \\
\text { PIE-Pre I }\end{array}$ & $\begin{array}{l}0.625 \\
0.626 \\
0.6224 \\
+0.001 \\
-0.0026\end{array}$ & $\begin{array}{l}0.625 \\
\text { ND } \\
0.6234 \\
\text { ND } \\
-0.0016\end{array}$ \\
\hline 155 & $\begin{array}{l}\text { Pre I } \\
\text { Robot } \\
\text { PIE } \\
\text { Robot-Pre I } \\
\text { PIE-Pre I }\end{array}$ & $\begin{array}{l}0.625 \\
0.623 \\
0.6227 \\
-0.002 \\
-0.0023\end{array}$ & $\begin{array}{l}0.624 \\
\text { ND } \\
0.6227 \\
\text { ND } \\
-0.0013\end{array}$ \\
\hline 170 & $\begin{array}{l}\text { Pre I } \\
\text { Robot } \\
\text { PIE } \\
\text { Robot-Pre I } \\
\text { PIE-Pre I }\end{array}$ & $\begin{array}{l}0.625 \\
0.624 \\
0.6229 \\
-0.001 \\
-0.0021\end{array}$ & $\begin{array}{l}0.624 \\
\text { ND } \\
0.6225 \\
\text { ND } \\
-0.0015\end{array}$ \\
\hline 303 & $\begin{array}{l}\text { Pre I } \\
\text { Robot } \\
\text { PIE } \\
\text { Robot-Pre I } \\
\text { PIE-Pre I }\end{array}$ & $\begin{array}{l}0.625 \\
0.623 \\
0.6224 \\
-0.002 \\
-0.0026\end{array}$ & $\begin{array}{l}0.624 \\
\text { ND } \\
0.6225 \\
\text { ND } \\
-0.0015\end{array}$ \\
\hline 312 & $\begin{array}{l}\text { Pre I } \\
\text { Robot } \\
\text { PIE } \\
\text { Robot-Pre I } \\
\text { PIE-Pre I }\end{array}$ & $\begin{array}{l}0.625 \\
0.624 \\
0.6227 \\
-0.001 \\
-0.0023\end{array}$ & $\begin{array}{l}0.624 \\
\text { ND } \\
0.6232 \\
\text { ND } \\
-0.0008\end{array}$ \\
\hline Robot & $\begin{array}{l}\text { Mean } \\
\text { Std Dev. }\end{array}$ & $\begin{array}{l}0.6242 \\
0.0012\end{array}$ & $\begin{array}{l}\text { ND } \\
\text { ND }\end{array}$ \\
\hline PIE & $\begin{array}{l}\text { Mean } \\
\text { Std Dev. }\end{array}$ & $\begin{array}{l}0.6227 \\
0.0002\end{array}$ & $\begin{array}{l}0.6230 \\
0.0004\end{array}$ \\
\hline Pre I & $\begin{array}{l}\text { Mean } \\
\text { Std Dev. }\end{array}$ & $\begin{array}{l}0.6250 \\
0\end{array}$ & $\begin{array}{l}0.6243 \\
0.0006\end{array}$ \\
\hline PIE-Pre I & $\begin{array}{l}\text { Mean } \\
\text { Std Dev. }\end{array}$ & $\begin{array}{r}-0.0024 \\
0.0002\end{array}$ & $\begin{array}{r}-0.0014 \\
0.0003\end{array}$ \\
\hline$\frac{\text { PIE-Pre I }}{\text { Pre }}$ & (\% strain) & -0.38 & -0.22 \\
\hline
\end{tabular}

(a) 1 in. $=25.4 \mathrm{~mm}$.

(b) See Fig. 2.5.

(c) Not determined. 
TABLE 4-5

FSV FUEL ELEXENT 1-0743

BOW OF SIX SIDE FriCES(a)

\begin{tabular}{|c|c|c|c|c|c|c|c|c|c|c|c|c|}
\hline \multirow[b]{2}{*}{ Position } & \multicolumn{2}{|c|}{ Face $A$} & \multicolumn{2}{|c|}{ Face B } & \multicolumn{2}{|c|}{ Face C } & \multicolumn{2}{|c|}{ Face D } & \multicolumn{2}{|c|}{ Face E } & \multicolumn{2}{|c|}{ Face F } \\
\hline & Robot & PIE & Robot & PIE & Robot & PIE & Robot & PIE & Robot & PIE & Robot & PIE \\
\hline $6^{(c)}$ & +0.0040 & +0.0036 & +0.0047 & +0.0047 & +0.0044 & $+0.002 i$ & $-0.00 i 6$ & $-0.00: 9$ & -0.0026 & -0.0034 & -0.0004 & -0.0018 \\
\hline 7 & +0.0049 & +0.0023 & +0.0064 & +0.0046 & +0.0042 & $+0.002 i$ & -0.0017 & -0.0018 & -0.0006 & -0.0031 & -0.0003 & -0.0020 \\
\hline 8 & +0.0050 & +0.0023 & +0.0074 & +0.0019 & +0.0042 & +0.0028 & -0.0008 & -0.0014 & -0.0024 & -0.0047 & -0.0012 & -0.0021 \\
\hline 9 & +0.0049 & +0.0026 & +0.0055 & +0.0043 & +0.0044 & $+0.003 i$ & -0.0007 & -0.0016 & -0.0025 & -0.0035 & -0.0002 & -0.0021 \\
\hline 10 & +0.0031 & +0.0019 & +0.0066 & +0.0047 & +0.0053 & +0.0032 & +0.0015 & -0.0009 & -0.0024 & -0.0034 & -0.0013 & -0.0021 \\
\hline 11 & +0.0052 & +0.0034 & +0.0112 & +0.0081 & +0.0076 & +0.0053 & 0 & -0.0017 & -0.0048 & -0.0063 & -0.0016 & -0.0039 \\
\hline 12 & +0.0068 & +0.0044 & +0.0100 & +0.0077 & +0.0058 & +0.0052 & +0.0006 & -0.0027 & -0.0040 & -0.0057 & -0.0024 & -0.0040 \\
\hline 13 & +0.0060 & +0.004 & +0.0118 & +0.0039 & +0.0074 & +0.0045 & $-0.00 i 6$ & -0.0027 & $-0.0 \cup 28$ & -0.0066 & -0.0024 & -0.0040 \\
\hline 14 & +0.0058 & +0.0037 & +0.0108 & +0.0074 & +0.0074 & +0.0044 & -0.0014 & -0.0033 & -0.0042 & -0.0059 & -0.0006 & -0.0033 \\
\hline 15 & +0.0070 & +0.0056 & +0.0094 & +0.0079 & +0.0068 & +0.0037 & -0.0022 & $-0.003 i$ & -0.0032 & -0.0066 & +0.0002 & -0.0030 \\
\hline 16 & +0.0090 & +0.0066 & +0.0121 & +0.0097 & +0.0092 & +0.0054 & -0.0028 & -0.0040 & -0.0058 & -0.0088 & +0.0008 & -0.0040 \\
\hline 17 & +0.0087 & +0.0048 & +0.0132 & +0.0093 & +0.0106 & +0.0055 & $-0.002 i$ & -0.0044 & -0.0048 & -0.0084 & -0.0009 & -0.0044 \\
\hline 18 & +0.0080 & +0.0055 & +0.0132 & +0.0058 & +0.0086 & +0.0058 & -0.0004 & -0.0034 & -0.0052 & -0.0096 & -0.0026 & -0.0052 \\
\hline 19 & +0.0087 & +0.0053 & +0.0125 & +0.0098 & +0.0092 & +0.0063 & -0.000 & -0.0032 & -0.0055 & -0.0084 & -0.0016 & -0.0051 \\
\hline 20 & +0.0073 & +0.0046 & +0.0128 & +0.0100 & +0.0089 & +0.0065 & +0.0005 & -0.0023 & -0.0062 & -0.0087 & -0.0019 & -0.0052 \\
\hline 21 & +0.0084 & +0.0054 & +0.0144 & $+0.01: 0$ & +0.0092 & +0.0073 & 0 & -0.0027 & -0.0066 & -0.0099 & -0.0032 & -0.0058 \\
\hline 22 & +0.0086 & +0.0060 & +0.0150 & +0.0111 & +0.0106 & +0.0068 & +0.0002 & -0.0035 & -0.0070 & -0.0100 & -0.0018 & -0.0056 \\
\hline 23 & +0.0090 & +0.0064 & +0.0146 & +0.0078 & +0.0098 & +0.0066 & -0.0002 & -0.0038 & -0.0066 & -0.0109 & -0.0018 & -0.0058 \\
\hline 24 & +0.0096 & +0.0058 & +0.0136 & +0.0106 & +0.0108 & +0.0061 & -0.0008 & -0.0049 & -0.0064 & -0.0098 & -0.0002 & -0.0052 \\
\hline 25 & +0.0100 & +0.0069 & +0.0138 & +0.0109 & +0.0096 & +0.0062 & -0.0014 & -0.0047 & -0.0064 & -0.0101 & +0.0004 & -0.0045 \\
\hline 26 & +0.0100 & +0.0070 & +0.0145 & +0.0114 & +0.0110 & +0.0064 & -0.0020 & -0.0047 & -0.0070 & -0.0107 & -0.0010 & -0.0042 \\
\hline 27 & +0.0095 & +0.0059 & +0.0150 & +0.0109 & +0.0110 & +0.0067 & -0.0015 & $-0.00: 5$ & -0.0060 & -0.0103 & -0.0015 & -0.0053 \\
\hline 28 & +0.0090 & +0.0058 & +0.0140 & +0.0097 & +0.0100 & +0.0069 & 0 & -0.0042 & -0.0060 & -0.0113 & -0.0020 & -0.0060 \\
\hline 29 & +0.0095 & +0.0060 & +0.0135 & +0.0109 & +0.0110 & +0.0070 & +0.0005 & -0.0039 & -0.0065 & -0.0105 & -0.0020 & -0.0060 \\
\hline 30 & +0.0085 & +0.0054 & +0.0140 & +0.0113 & +0.0095 & +0.0074 & +0.0015 & -0.0028 & -0.0070 & $-0.0: 04$ & -0.0035 & -0.0064 \\
\hline 31 & +0.0086 & +0.0053 & +0.0136 & +0.0106 & +0.0088 & +0.0068 & +0.0010 & -0.0026 & -0.0064 & -0.0097 & -0.0028 & -0.0059 \\
\hline 32 & +0.0074 & +0.0057 & +0.0130 & +0.0101 & +0.0094 & +0.0065 & -0.0002 & -0.0036 & -0.0060 & -0.0099 & -0.0032 & -0.0056 \\
\hline 33 & +0.0080 & +0.0057 & +0.0124 & $+0.011 i$ & +0.0082 & +0.0064 & -0.0018 & -0.0035 & -0.0064 & -0.0108 & -0.0032 & -0.0052 \\
\hline 34 & +0.0084 & +0.0056 & +0.0124 & +0.0104 & +0.0092 & +0.0063 & -0.0012 & -0.0049 & -0.0066 & -0.0098 & -0.0008 & -0.0046 \\
\hline 35 & +0.0090 & +0.0066 & +0.0122 & +0.0104 & +0.0104 & +0.0059 & -0.0016 & -0.0042 & -0.0066 & -0.0097 & +0.0046 & -0.0039 \\
\hline 36 & +0.0070 & +0.0056 & +0.0089 & +0.0088 & +0.0088 & +0.0050 & -0.0022 & $-0.004 i$ & -0.0062 & -0.0084 & -0.0008 & -0.0028 \\
\hline 37 & +0.0073 & +0.0051 & +0.0098 & +0.0083 & +0.0074 & +0.0052 & -0.0019 & $-0.004 i$ & -0.0062 & -0.0083 & $-0.002 i$ & -0.0042 \\
\hline 38 & +0.0060 & +0.0049 & +0.0098 & +0.0092 & +0.0064 & +0.0052 & -0.0016 & -0.0037 & -0.0058 & -0.0089 & -0.0024 & -0.0048 \\
\hline 39 & +0.0073 & +0.0050 & +0.0105 & +0.0081 & +0.0068 & +0.0055 & -0.0009 & $-0.003 ?$ & -0.0065 & -0.0081 & -0.0024 & -0.0036 \\
\hline 40 & +0.0057 & +0.0046 & +0.0102 & +0.0089 & $+0.006 i$ & +0.0059 & -0.0005 & $-0.002 i$ & -0.0058 & -0.0080 & $-0.003 i$ & -0.0053 \\
\hline 41 & +0.0038 & +0.0032 & +0.0068 & +0.0058 & +0.0046 & +0.006 & o & -0.0012 & -0.0032 & -0.0052 & -0.0034 & -0.0036 \\
\hline 42 & +0.0032 & +0.0036 & +0.0070 & +0.0054 & +0.0052 & +0.0036 & -0.0006 & -0.0022 & -0.0050 & -0.0053 & -0.0016 & -0.0020 \\
\hline 43 & +0.0040 & +0.0036 & +0.0062 & +0.0063 & +0.0046 & +0.0040 & -0.0024 & -0.0026 & -0.0032 & -0.0056 & -0.0026 & -0.0033 \\
\hline 44 & +0.0052 & +0.0037 & +0.0072 & +0.0053 & +0.0046 & +0.0039 & -0.0016 & $-0.003 i$ & -0.0048 & -0.0055 & $-0.00 i 4$ & -0.0027 \\
\hline 45 & +0.0040 & +0.0040 & +0.0056 & +0.0060 & +0.0052 & +0.0035 & -0.0008 & -0.0025 & -0.0058 & -0.0061 & -0.0002 & -0.0019 \\
\hline 46 & +0.0020 & +0.0018 & +0.0013 & +0.0025 & +0.0016 & +0.0014 & -0.0014 & -0.0006 & -0.0032 & -0.0025 & -0.0006 & -0.0003 \\
\hline 47 & $+0.002 i$ & +0.0017 & +0.0026 & +0.0023 & +0.0008 & +0.0018 & -0.0013 & -0.0010 & -0.0014 & -0.0020 & -0.0007 & -0.0007 \\
\hline 48 & +0.0010 & +0.0017 & +0.0026 & +0.0028 & +0.0008 & +0.0017 & -0.0012 & -0.0008 & -0.0006 & -0.0025 & +0.0022 & -0.0012 \\
\hline 49 & +0.0013 & +0.0018 & +0.0025 & +0.0021 & +0.0016 & +0.0018 & -0.0003 & -0.0006 & -0.0025 & -0.0019 & -0.0008 & -0.0011 \\
\hline 50 & +0.0019 & +0.0017 & +0.0024 & -0.0021 & -0.0003 & +0.0017 & +0.0005 & 0 & -0.0016 & -0.0014 & -0.0017 & -0.0015 \\
\hline $\operatorname{Mean}(d)$ & +0.0064 & +0.0045 & +0.0099 & +0.0075 & +0.0070 & +0.0049 & -0.0008 & -0.0029 & -0.0048 & -0.0073 & $-0.00 i 3$ & -0.0038 \\
\hline Std. Dev. & 0.0026 & 0.0016 & 0.0040 & 0.0033 & 0.0031 & 0.0018 & 0.0010 & 0.0013 & 0.0019 & 0.0029 & 0.0015 & 0.0016 \\
\hline
\end{tabular}

(a) See detail $T$ in Fig. 2-5 for interpretation of + and - values.

(b) 1 in. $=25.4 \mathrm{~mm}$.

(c) Positions 1-5 and 5i-55 have zero values.

(d) Excluding 1-5 and 51-55. 
TABLE $4-6$

FSV FUEL, ELEMENT 1-0743

DISPLACEMENT FRUM VERTICAL OF SIX SIDE FACES (a) (inches) (b)

\begin{tabular}{|c|c|c|c|c|c|c|c|c|c|c|c|c|}
\hline \multirow[b]{2}{*}{ Position } & \multicolumn{2}{|c|}{ Face A } & \multicolumn{2}{|c|}{ Face $B$} & \multicolumn{2}{|c|}{ Face $\mathrm{C}$} & \multicolumn{2}{|c|}{ Face D } & \multicolumn{2}{|c|}{ Face E } & \multicolumn{2}{|c|}{ Face $F$} \\
\hline & Robot & PIE & Robot & PIE & Robot & PIE & Robot & PIE & Robot & PIE & Kobot & PIE \\
\hline $6^{(c)}$ & +0.002 & +0.0030 & +0.001 & +0.0023 & +0.003 & +0.0008 & 0 & +0.0002 & +0.001 & +0.0003 & +0.001 & +0.0001 \\
\hline 7 & +0.003 & +0.0015 & +0.003 & +0.0023 & +0.003 & +0.0008 & 0 & +0.0004 & +0.003 & +0.0004 & +0.001 & +0.0001 \\
\hline 8 & +0.003 & +0.0014 & +0.004 & 0 & +0.003 & +0.0015 & +0.001 & +0.0008 & +0.001 & -0.0013 & 0 & 0 \\
\hline 9 & +0.003 & +0.0018 & +0.002 & +0.0021 & +0.003 & +0.0019 & +0.001 & +0.0005 & +0.001 & 0 & +0.001 & +0.0001 \\
\hline 10 & +0.001 & +0.0009 & +0.003 & +0.0023 & +0.004 & +0.0020 & +0.003 & +0.0011 & +0.001 & +0.0001 & 0 & 0 \\
\hline 11 & +0.001 & +0.0015 & +0.004 & +0.0033 & +0.005 & +0.0029 & +0.003 & +0.0022 & +0.002 & +0.0007 & +0.001 & +0.0002 \\
\hline 12 & +0.003 & +0.0027 & +0.003 & +0.0032 & +0.003 & +0.0029 & +0.004 & +0.0015 & +0.003 & +0.0013 & 0 & +0.0003 \\
\hline 13 & +0.002 & +0.0023 & +0.005 & 0 & +0.005 & +0.0020 & +0.002 & +0.0016 & +0.004 & +0.0002 & 0 & +0.0003 \\
\hline 14 & +0.002 & +0.0021 & +0.004 & +0.0028 & +0.005 & +0.0017 & +0.002 & +0.0010 & +0.003 & +0.0012 & +0.002 & +0.0009 \\
\hline 15 & +0.003 & +0.0044 & +0.002 & +0.0032 & +0.004 & +0.0011 & +0.001 & +0.0011 & +9.004 & +0.0008 & +0.003 & +0.0009 \\
\hline 16 & +0.003 & +0.0048 & +0.001 & +0.0026 & +0.005 & +0.0015 & +0.002 & +0.0022 & +0.005 & +0.0023 & +0.005 & +0.0018 \\
\hline 17 & +0.003 & +0.0024 & +0.003 & +0.0025 & +0.007 & +0.0015 & +0.003 & +0.0021 & +0.006 & +0.0022 & +0.003 & +0.0019 \\
\hline 18 & +0.002 & +0.0028 & +0.003 & 0 & +0.005 & +0.0020 & +0.005 & +0.0031 & +0.005 & +0.0006 & +0.001 & +0.0012 \\
\hline 19 & +0.003 & +0.0028 & +0.002 & +0.0031 & +0.005 & +0.0028 & +0.005 & +0.0031 & +0.005 & +0.0020 & +0.002 & +0.0014 \\
\hline 20 & +0.001 & +0.0017 & +0.002 & +0.0028 & +0.005 & +0.0029 & +0.005 & +0.0036 & +0.004 & +0.0018 & +0.002 & +0.0010 \\
\hline 21 & 0 & +0.0016 & 0 & +0.0014 & +0.004 & +0.0025 & +0.006 & +0.0051 & +0.007 & +0.0041 & +0.002 & +0.0025 \\
\hline 22 & +0.001 & +0.0026 & +0.001 & +0.0022 & +0.005 & +0.0022 & +0.007 & +0.0049 & +0.007 & +0.0039 & +0.003 & +0.0030 \\
\hline 23 & +0.001 & +0.0028 & +0.001 & 0 & +0.005 & +0.0016 & +0.007 & +0.0048 & +0.007 & +0.0027 & +0.003 & +0.0028 \\
\hline 24 & +0.002 & +0.0026 & 0 & +0.0015 & +0.006 & +0.0008 & +0.006 & +0.0037 & +0.008 & +0.0044 & +0.005 & +0.0032 \\
\hline 25 & +0.002 & +0.0045 & -0.001 & +0.0014 & +0.004 & +0.0010 & +0.005 & +0.0036 & +0.008 & +0.0047 & +0.006 & +0.0033 \\
\hline 26 & 0 & +0.0039 & -0.004 & -0.0005 & +0.004 & -0.0002 & +0.006 & +0.0077 & +0.011 & +0.0078 & +0.006 & +0.0055 \\
\hline 27 & 0 & +0.0018 & -0.002 & -0.0005 & +0.005 & 0 & +0.007 & +0.0058 & +0.012 & +0.0074 & +0.005 & +0.0052 \\
\hline 28 & -0.001 & +0.0013 & -0.003 & 0 & +0.004 & +0.0006 & +0.009 & +0.0066 & +0.011 & +0.0057 & +0.004 & +0.0047 \\
\hline 29 & 0 & +0.0018 & -0.004 & -0.0003 & +0.004 & +0.0012 & +0.009 & +0.0066 & +0.011 & +0.0069 & +0.004 & +0.0048 \\
\hline 30 & -0.002 & +0.0006 & -0.004 & -0.0007 & +0.003 & +0.0014 & +0.009 & +0.0070 & +0.010 & +0.0071 & +0.003 & $+0,0040$ \\
\hline 31 & -0.004 & -0.0004 & -0.008 & -0.0038 & +0.001 & -0.0004 & +0.010 & +0.0091 & +0.014 & +0.0112 & +0.005 & +0.0065 \\
\hline 32 & -0.004 & +0.0007 & -0.008 & -0.0033 & +0.001 & -0.0004 & +0.010 & +0.0090 & +0.015 & +0.0110 & +0.004 & +0.0073 \\
\hline 33 & -0.004 & +0.0003 & -0.008 & -0.0005 & +0.001 & -0.0011 & +0.009 & +0.0095 & +0.014 & +0.0095 & +0.004 & +0.0076 \\
\hline 34 & -0.003 & +0.0007 & -0.008 & -0.0033 & +0.002 & -0.0017 & +0.009 & +0.0080 & +0.015 & +0.0114 & +0.007 & +0.0079 \\
\hline 35 & -0.003 & +0.0029 & -0.010 & -0.0038 & +0.002 & -0.0020 & +0.008 & +0.0083 & +0.015 & +0.0124 & +0.013 & +0.0077 \\
\hline 36 & -0.001 & +0.0013 & -0.017 & -0.0078 & -0.001 & -0.0042 & +0.009 & +0.0105 & +0.019 & +0.0174 & +0.009 & +0.0108 \\
\hline 37 & -0.006 & -0.0006 & -0.014 & -0.0077 & -0.001 & -0.0041 & +0.010 & +0.0110 & +0.019 & +0.0165 & +0.007 & +0.0104 \\
\hline 38 & -0.008 & -0.0014 & -0.014 & -0.0044 & -0.002 & -0.0036 & +0.011 & +0.0114 & +0.018 & +0.0148 & +0.006 & +0.0102 \\
\hline 39 & -0.006 & -0.0009 & -0.014 & -0.0075 & -0.003 & -0.0026 & $+0.0 ! 1$ & +0.0115 & +0.018 & +0.0127 & +0.006 & +0.0115 \\
\hline 40 & -0.009 & -0.0021 & -0.015 & -0.0079 & -0.003 & -0.0025 & +0.010 & +0.0116 & +0.018 & +0.0164 & +0.006 & +0.0092 \\
\hline $4:$ & -0.013 & -0.0044 & -0.022 & -0.0134 & -0.006 & -0.0055 & +0.012 & +0.0144 & +0.024 & +0.0227 & +0.007 & +0.0130 \\
\hline 42 & -0.012 & -0.0031 & -0.021 & -0.0124 & -0.006 & -0.0056 & +0.013 & +0.0146 & +0.023 & +0.0225 & +0.008 & +0.0152 \\
\hline 43 & -0.012 & -0.0036 & -0.021 & -0.0092 & -0.005 & -0.0060 & +0.012 & +0.0147 & +0.024 & +0.0215 & +0.007 & +0.0138 \\
\hline 44 & -0.010 & -0.0028 & -0.020 & -0.0129 & -0.005 & -0.0067 & +0.012 & +0.0141 & +0.024 & +0.0228 & +0.009 & +0.0140 \\
\hline 45 & -0.012 & -0.0009 & -0.024 & -0.0130 & -0.006 & -0.0070 & +0.012 & +0.0141 & +0.023 & +0.0234 & +0.011 & +0.0136 \\
\hline 46 & -0.016 & -0.0037 & -0.032 & -0.0188 & -0.011 & -0.0104 & +0.013 & +0.0181 & +0.031 & +0.0307 & +0.012 & +0.0172 \\
\hline 47 & -0.015 & -0.0056 & -0.028 & -0.0182 & -0.010 & -0.0102 & +0.014 & +0.0184 & +0.031 & +0.0299 & +0.011 & +0.0181 \\
\hline 48 & -0.017 & -0.0064 & -0.028 & -0.0147 & -0.010 & -0.0096 & +0.015 & +0.0186 & +0.030 & +0.0280 & +0.013 & +0.0181 \\
\hline 49 & -0.016 & -0.0058 & -0.029 & -0.0180 & -0.011 & -0.0086 & +0.015 & +0.0183 & +0.029 & +0.0294 & +0.010 & +0.0183 \\
\hline 50 & -0.017 & -0.0069 & -0.030 & -0.0195 & -0.012 & -0.0091 & +0.014 & +0.0176 & +0.029 & +0.0300 & +0.010 & +0.0171 \\
\hline 51 & -0.021 & -0.0095 & -0.036 & -0.0240 & -0.013 & -0.0120 & +0.015 & +0.0195 & +0.034 & +0.0349 & +0.013 & +0.0207 \\
\hline 52 & -0.019 & -0.0084 & -0.035 & -0.0223 & -0.014 & -0.0115 & +0.017 & +0.0210 & +0.035 & +0.0348 & +0.012 & +0.0215 \\
\hline 53 & -0.020 & -0.0090 & -0.034 & -0.0194 & -0.012 & -0.0125 & +0.018 & +0.0216 & +0.034 & +0.0339 & +0.012 & +0.0214 \\
\hline 54 & -0.019 & -0.0081 & -0.034 & -0.0228 & -0.012 & -0.0133 & +0.017 & +0.0215 & +0.036 & +0.0354 & +0.013 & +0.0209 \\
\hline 55 & -0.020 & -0.0061 & -0.037 & -0.0237 & -0.014 & -0.0131 & +0.016 & +0.0208 & +0.036 & +0.0369 & +0.014 & +0.0194 \\
\hline Nean $(d)$ & -0.0050 & -0.0005 & -0.0104 & -0.0055 & -0.0008 & -0.0025 & +0.0084 & +0.0090 & +0.0150 & +0.0127 & +0.0058 & +0.0080 \\
\hline Std. Dev. & 0.0080 & 0.0039 & 0.0136 & 0.0088 & 0.0065 & 0.0051 & 0.0050 & 0.0068 & 0.0111 & 0.0121 & 0.0042 & 0.0071 \\
\hline
\end{tabular}

(a) See detail $T$ in Fig. 2-5 for interpretation of + and - values.

(b) 1 in. $=25.4 \mathrm{~mm}$.

(c) Positions 1-5 have zero values.

(d) Excluding 1-5. 

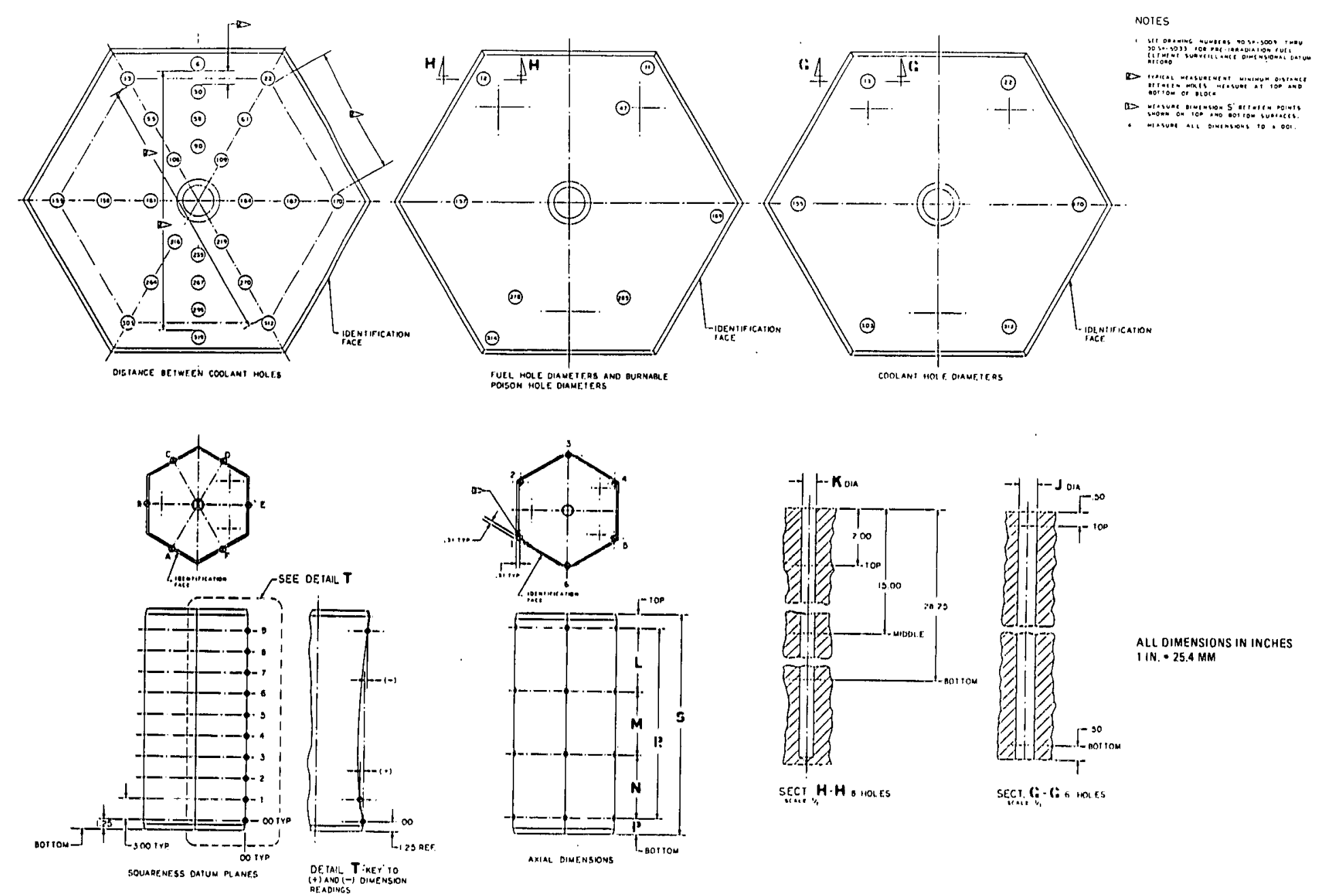

ALL DIMENSIONS IN INCHES
IIN. 25.4 MM

Fig. 2-5. Prefrradiation fuel block measurements for FSV fuel.

- element 1-0743

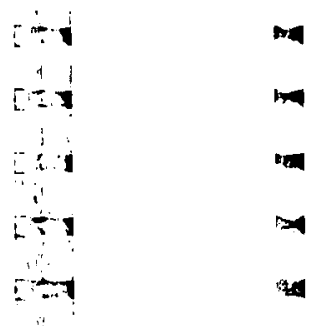

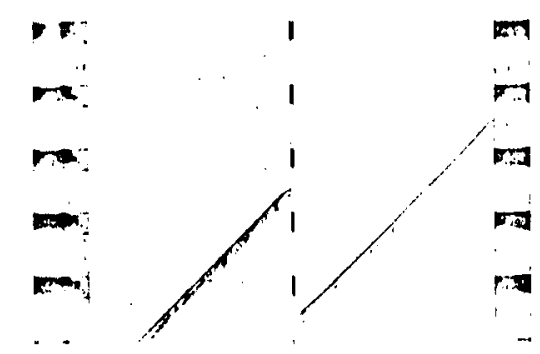

$m$
$m$
$n$
$n$

+1
+1
+1

$\begin{array}{ll}\cdots 1 & 1 \\ 1 & 1 \\ \cdots 1 & 1 \\ \cdots & 1\end{array}$


TABLE 4-7

FSV FUEL ELEMENT 1-0743

DISTANCES ACROSS FLATS

(inches) (a)

\begin{tabular}{|c|c|c|c|c|c|c|}
\hline Face (b) & Meas. & $1-2$ & $3-4$ & $5-6$ & Mean & Std Dev. \\
\hline$B-E$ & $\begin{array}{l}\text { Pre I } \\
\text { CC (d) } \\
\text { Robot } \\
\text { PIE } \\
\text { PIE-CC } \\
\text { Pre I } \\
\text { CC (d) } \\
\text { Robot } \\
\text { PIE } \\
\text { PIE-CC } \\
\text { Pre I } \\
\text { CC (d) } \\
\text { Robot } \\
\text { PIE } \\
\text { PIE-CC }\end{array}$ & $\begin{array}{l}14.147 \\
14.148 \\
14.151 \\
14.157 \\
\\
14.153 \\
14.150 \\
14.154 \\
14.154 \\
\\
14.150 \\
14.157 \\
14.159 \\
14.151\end{array}$ & $\begin{array}{l}14.150 \\
14.150 \\
14.154 \\
14.153 \\
\\
14.151 \\
14.154 \\
14.157 \\
14.152\end{array}$ & $\begin{array}{l}14.1772(\mathrm{c}) \\
14.1730 \\
14.149 \\
14.155 \\
14.154 \\
-0.0190 \\
14.1756(\mathrm{~d}) \\
14.1714 \\
14.152 \\
14.150 \\
14.154 \\
-0.0174 \\
14.1769(\mathrm{~d}) \\
14.1714 \\
14.154 \\
14.153 \\
14.154 \\
-0.0187\end{array}$ & $\begin{array}{l}14.1508 \\
14.1538 \\
14.1532 \\
14.1546\end{array}$ & $\begin{array}{l}0.0013 \\
0.0005\end{array}$ \\
\hline Robot & $\begin{array}{l}\text { Mean } \\
\text { Std Dev. }\end{array}$ & $\begin{array}{r}14.1525 \\
0.0043\end{array}$ & $\begin{array}{r}14.1518 \\
0.0026\end{array}$ & $\begin{array}{r}14.1522 \\
0.0023\end{array}$ & & \\
\hline $\begin{array}{l}\text { PIE } \\
\text { CC (b). }\end{array}$ & $\begin{array}{l}\text { Mean } \\
\text { Std Dev. } \\
\text { Mean } \\
\text { Std Dev. }\end{array}$ & $\begin{array}{c}14.1543 \\
0.0032 \\
\mathrm{ND}^{(\mathrm{e})}\end{array}$ & $\begin{array}{r}14.1538 \\
0.0023 \\
\text { ND }\end{array}$ & $\begin{array}{l}14.1540 \\
0 \\
14.1724 \\
0.0008\end{array}$ & & \\
\hline $\begin{array}{l}\text { PIE-Pre } \\
\frac{\text { PIE-Pre }}{\text { Pre I }}\end{array}$ & $\begin{array}{l}\text { I Mean } \\
\text { Std Dev. } \\
\text { I ( } \% \text { strai }\end{array}$ & ND & $\mathrm{ND}$ & $\begin{array}{c}-0.0184 \\
0.0009 \\
-0.13 \%\end{array}$ & & \\
\hline
\end{tabular}
(a) ${ }_{1}$ in. $=25.4 \mathrm{~mm}$.
(b) See Fig. 2-5.
(c) Cordax.
(d) Cordax corrected (CC).
(e) Not determined. 
TABLE 4-8

FSV FUEL ELEMENT 1-0743 LENGTH

(inches) (a)

\begin{tabular}{|c|c|c|c|c|c|c|}
\hline Meas. & Location (b) & Pre Char & Robot & PIE & $\begin{array}{c}\text { Corners } \\
\text { Only }\end{array}$ & $\begin{array}{r}\text { PIE - } \\
\text { Robot }\end{array}$ \\
\hline 1 & $C-324$ & 31.2345 & 31.150 & 31.170 & 31.169 & +0.020 \\
\hline 2 & $321-322$ & $\mathrm{ND}^{(\mathrm{c})}$ & 31.154 & 31.169 & & +0.015 \\
\hline 3 & S-319 & ND & 31.147 & 31.168 & & +0.021 \\
\hline 4 & $316-317$ & ND & 31.148 & 31.168 & & +0.020 \\
\hline 5 & $C-314$ & 31.2330 & 31.147 & 31.167 & 31.165 & +0.020 \\
\hline 6 & $275-289$ & $\mathrm{ND}$ & 31.147 & 31.170 & & +0.023 \\
\hline 7 & $293-294$ & ND & 31.155 & 31.171 & & +0.016 \\
\hline 8 & $296-297$ & ND & 31.154 & 31.173 & & +0.019 \\
\hline 9 & $288-301$ & ND & 31.163 & 31.173 & & +0.010 \\
\hline 10 & S-259 & ND & 31.163 & 31.175 & & +0.012 \\
\hline 11 & $257-272$ & ND & 31.164 & 31.176 & & +0.012 \\
\hline 12 & $237-254$ & ND & 31.162 & 31.178 & & +0.016 \\
\hline 13 & $251-252$ & ND & 31.160 & ND & & ND \\
\hline 14 & $233-249$ & $\mathrm{ND}$ & 31.156 & 31.178 & & +0.022 \\
\hline 15 & $246-262$ & ND & 31.153 & 31.175 & & +0.022 \\
\hline 16 & S-244 & ND & 31.155 & 31.173 & & +0.018 \\
\hline 17 & $190-209$ & ND & 31.156 & 31.178 & & +0.022 \\
\hline 18 & $192-211$ & ND & 31.162 & 31.179 & & +0.017 \\
\hline 19 & $195-214$ & ND & 31.162 & 31.181 & & +0.019 \\
\hline 20 & $203-221$ & ND & 31.166 & 31.181 & & +0.015 \\
\hline 21 & $206-224$ & ND & 31.168 & 31.180 & & +0.012 \\
\hline 22 & $208-226$ & ND & 31.166 & 31.179 & & +0.013 \\
\hline 23 & $C-171$ & 31.2330 & 31.164 & 31.182 & 31.180 & +0.018 \\
\hline 24 & $165-166$ & ND & 31.163 & 31.182 & & +0.019 \\
\hline 25 & $\mathrm{HH}-163$ & ND & 31.169 & ND & & ND \\
\hline 26 & $\mathrm{HH}-200$ & ND & 31.161 & ND & & ND \\
\hline 27 & $\mathrm{HH}-198$ & ND & 31.164 & ND & & ND \\
\hline 28 & $\mathrm{HH}-127$ & ND & 31.166 & ND & & ND \\
\hline 29 & HH-125 & ND & 31.167 & ND & & ND \\
\hline 30 & $\mathrm{HH}-162$ & ND & 31.162 & ND & & ND \\
\hline 31 & $159-160$ & ND & 31.165 & 31.184 & & +0.019 \\
\hline 32 & $C-154$ & 31.2330 & 31.159 & 31.182 & 31.180 & +0.021 \\
\hline 33 & $99-117$ & ND & 31.173 & 31.185 & & +0.012 \\
\hline 34 & $101-119$ & ND & 31.174 & 31.189 & & +0.015 \\
\hline 35 & $104-122$ & ND & 31.171 & ND & & ND \\
\hline 36 & $111-130$ & ND & 31.167 & ND & & ND \\
\hline 37 & $114-133$ & ND & 31.173 & 31.189 & & +0.016 \\
\hline 38 & $116-135$ & IID & 31.169 & 31.186 & & +0.017 \\
\hline 39 & $S-81$ & ND & 31.174 & 31.190 & & +0.016 \\
\hline 40 & $63-79$ & ND & 31.173 & 31.192 & & +0.019 \\
\hline 41 & $76-92$ & ND & 31.171 & ND & & ND \\
\hline 42 & $73-74$ & ND & 31.172 & ND & & ND \\
\hline 43 & $71-88$ & ND & 31.178 & ND & & ND \\
\hline 44 & $53-68$ & ND & 31.179 & 31.193 & & +0.014 \\
\hline 45 & $S-66$ & ND & 31.175 & 31.190 & & +0.015 \\
\hline 46 & $24-37$ & ND & 31.183 & 31.192 & & +0.009 \\
\hline 47 & $28-29$ & ND & 31.178 & 31.196 & & +0.018 \\
\hline 48 & $31-32$ & ND & 31.180 & 31.197 & & +0.017 \\
\hline 49 & $36-50$ & ND & 31.177 & 31.193 & & +0.016 \\
\hline 50 & $C-11$ & 31.2315 & 31.182 & 31.197 & 31.196 & +0.014 \\
\hline 51 & $8-9$ & ND & 31.180 & 31.196 & & +0.016 \\
\hline 52 & $S-6$ & ND & 31.182 & 31.191 & & +0.009 \\
\hline 53 & $3-4$ & ND & 31.185 & 31.196 & & +0.011 \\
\hline 54 & $C-1$ & 31.2320 & 31.182 & 31.197 & 31.195 & +0.013 \\
\hline Mean & & & 31.1662 & 31.1824 & & +0.0165 \\
\hline d. Dev. & & & 0.0103 & 0.0095 & & +0.0037 \\
\hline
\end{tabular}

(a) 1 in. $=25.4 \mathrm{~mm}$.

(b) $\mathrm{C}=$ corner of element; $\mathrm{S}=$ side of element; $\mathrm{HH}=$ handling hole.

For example, $\mathrm{C}-324=$ between corner and hole number 324 .

(c) $\mathrm{ND}=$ not determined. 
TABLE 4-9

FSV FUEL ELEMENT 1-0743

DISTANCES BETWEEN CENTERLINES OF COOLANT HOLES

(inches) (a)

\begin{tabular}{|c|c|c|c|c|c|c|c|c|}
\hline Hole & to Hole & $\begin{array}{l}\text { Pre I } \\
\text { Top }\end{array}$ & $\begin{array}{l}\text { Pre I } \\
\text { Bottom }\end{array}$ & $\begin{array}{l}\text { Robot } \\
\text { Top }\end{array}$ & $\begin{array}{l}\text { PIE } \\
\text { TOP }\end{array}$ & $\begin{array}{c}\text { PIE } \\
\text { Bottom }\end{array}$ & $\begin{array}{l}\text { PIE - } \\
\text { Pre I }\end{array}$ & $\frac{\%}{\text { Strain }}$ \\
\hline 259 & -222 & $\mathrm{ND}^{(\mathrm{b})}$ & ND & 2.5601 & 2.556 & 2.566 & & \\
\hline 222 & -181 & ND & ND & 2.5657 & 2.561 & 2.576 & & \\
\hline 181 & -144 & ND & ND & 2.5570 & 2.563 & 2.561 & & \\
\hline 144 & -103 & ND & ND & 2.5636 & 2.562 & 2.556 & & \\
\hline 103 & -66 & ND & ND & 2.5602 & 2.556 & 2.563 & & \\
\hline 259 & -66 & ND & ND & 12.8051 & 12.797 & 12.821 & & \\
\hline 312 & -270 & ND & ND & 2.2185 & 2.214 & 2.215 & & \\
\hline 270 & -219 & ND & ND & 2.2181 & 2.220 & 2.220 & & \\
\hline 219 & -106 & ND & ND & 4.4394 & 4.436 & 4.442 & & \\
\hline 106 & -55 & ND & ND & 2.2181 & 2.217 & 2.225 & & \\
\hline 55 & -13 & ND & ND & 2.2201 & 2.217 & 2.219 & & \\
\hline 312 & -.13 & 13.3200 & 13.3245 & 13.3149 & 13.304 & 13.321 & $\begin{array}{l}+0.0160 \text { (top) } \\
-0.0035 \text { (bot) }\end{array}$ & $\begin{array}{l}-0.12 \\
-0.03\end{array}$ \\
\hline 319 & -295 & ND & ND & 1.2710 & 1.277 & 1.282 & & \\
\hline 295 & -267 & ND & ND & 1.2900 & 1.283 & 1.284 & & \\
\hline 267 & -235 & ND & ND & 1.2640 & 1.279 & 1.279 & & \\
\hline 235 & -199 & ND & ND & 1.2930 & 1.279 & 1.298 & & \\
\hline 199 & -126 & ND & ND & 2.5722 & 2.562 & 2.547 & . & \\
\hline 126 & -90 & ND & ND & 1.2750 & 1.280 & 1.287 & & \\
\hline 90 & -58 & ND & ND & 1.2830 & 1.283 & 1.280 & & \\
\hline 58 & -30 & ND & ND & 1.2740 & 1.277 & 1.274 & & \\
\hline 30 & -6 & ND & ND & 1.2840 & 1.281 & 1.287 & & \\
\hline 319 & -6 & ND & ND & 12.8063 & 12.801 & 12.818 & & \\
\hline 303 & -264 & ND & ND & 2.2130 & 2.217 & 2.220 & & \\
\hline 264 & -216 & ND & ND & 2.2147 & 2.217 & 2.222 & & \\
\hline 216 & -109 & ND & ND & 4.4437 & 4.437 & 4.447 & & \\
\hline 109 & -61 & ND & ND & 2.2113 & 2.216 & 2.221 & & \\
\hline 61 & -22 & ND & ND & 2.2130 & 2.216 & 2.217 & & \\
\hline 303 & -22 & ND & ND & 13.2947 & 13.303 & 13.327 & & \\
\hline 244 & -213 & ND & ND & 2.5561 & 2.558 & 2.562 & & \\
\hline 213 & -180 & ND & ND & 2.5762 & 2.561 & 2.567 & & \\
\hline 180 & -145 & ND & ND & 2.5570 & 2.564 & 2.560 & & \\
\hline 145 & -112 & ND & ND & 2.5534 & 2.558 & 2.570 & & \\
\hline 112 & -81 & ND & ND & 2.5555 & 2.558 & 2.568 & & \\
\hline 224 & -81 & ND & ND & 12.7975 & 12.800 & 12.827 & & \\
\hline 170 & -167 & ND & ND & 2.2110 & 2.216 & 2.216 & & \\
\hline 167 & -164 & ND & ND & 2.2150 & 2.214 & 2.219 & & \\
\hline 164 & -161 & ND & ND & 4.4510 & 4.439 & 4.440 & & \\
\hline 161 & -158 & ND & ND & 2.2180 & 2.216 & 2.220 & & \\
\hline 158 & -155 & ND & ND & 2.2151 & 2.216 & 2.218 & & \\
\hline 170 & -155 & ND & ND & 13.3091 & 13.301 & 13.312 & & \\
\hline 13 & -22 & 6.6620 & 6.6610 & 6.6545 & 6.655 & 6.665 & $\begin{array}{ll}-0.0070 & (\text { top }) \\
+0.0040 & \text { (bot) }\end{array}$ & $\begin{array}{l}-0.11 \\
+0.06\end{array}$ \\
\hline 22 & -170 & 6.6610 & 6.6615 & 6.6520 & 6.645 & 6.663 & $\begin{array}{l}-0.0160 \text { (top) } \\
+0.0015 \text { (bot) }\end{array}$ & $\begin{array}{l}-0.24 \\
+0.02\end{array}$ \\
\hline 170 & -312 & 6.6590 & 6.6590 & 6.6490 & 6.647 & 6.656 & $\begin{array}{l}-0.0120 \text { (top) } \\
-0.0030 \text { (bot) }\end{array}$ & $\begin{array}{l}-0.18 \\
-0.05\end{array}$ \\
\hline 312 & -305 & 6.6610 & 6.6590 & 6.6496 & 6.651 & 6.657 & $\begin{array}{ll}-0.010 & \text { (top) } \\
-0.002 & \text { (bot) }\end{array}$ & $\begin{array}{l}-0.15 \\
-0.03\end{array}$ \\
\hline 303 & -133 & 6.6600 & 6.6620 & 6.6516 & 6.650 & 6.657 & $\begin{array}{ll}-0.010 & \text { (top) } \\
-0.005 & \text { (bot) }\end{array}$ & $\begin{array}{l}-0.15 \\
-0.08\end{array}$ \\
\hline & -13 & 6.6630 & 6.6615 & 6.6490 & 6.654 & 6.665 & $\begin{array}{ll}-0.009 & \text { (top) } \\
+0.0035 & \text { (bot) }\end{array}$ & $\begin{array}{l}-0.14 \\
+0.05\end{array}$ \\
\hline $\begin{array}{r}M \\
\text { Std }\end{array}$ & $\begin{array}{l}\text { lean } \\
\text {. Dev. }\end{array}$ & & & $\begin{array}{l}4.2731 \\
3.8059\end{array}$ & $\begin{array}{l}4.2720 \\
3.8047\end{array}$ & & Top, bottom & $\begin{array}{l}-0.16, \\
+0.01\end{array}$ \\
\hline
\end{tabular}

(a) 1 in. $=25.4 \mathrm{~mm}$.

(b) $N D=$ not determined. 
TABLE 4-10

FSV FUEL ELEMENT 1-0743

COOLANT HOLE DIAMETERS

(inches) (a)

\begin{tabular}{|c|c|c|c|c|c|}
\hline Hole & $\begin{array}{c}\text { Pre I } \\
\text { Top }\end{array}$ & $\begin{array}{r}\text { Pre I } \\
\text { Bottom }\end{array}$ & $\begin{array}{c}\text { Robot } \\
\text { Top }\end{array}$ & $\begin{array}{l}\text { PIE } \\
\text { Top }\end{array}$ & $\begin{array}{c}\text { PIE } \\
\text { Bottom }\end{array}$ \\
\hline 6 & $\mathrm{ND}{ }^{(b)}$ & ND & 0.625 & 0.6227 & 0.6237 \\
\hline 13 & 0.625 & 0.625 & 0.625 & 0.6228 & 0.6234 \\
\hline 22 & 0.625 & 0.625 & 0.626 & 0.6224 & 0.6234 \\
\hline 30 & ND & ND & 0.624 & 0.6227 & 0.6232 \\
\hline 55 & ND & ND & 0.623 & 0.6225 & 0.6235 \\
\hline 58 & $\mathrm{ND}$ & ND & 0.625 & 0.6228 & 0.6235 \\
\hline 61 & ND & ND & 0.625 & 0.6224 & 0.6236 \\
\hline 66 & ND & $\mathrm{ND}$ & 0.624 & 0.6234 & 0.6232 \\
\hline 81 & ND & ND & 0.624 & 0.6227 & 0.6234 \\
\hline 90 & ND & ND & 0.624 & 0.6231 & 0.6236 \\
\hline 103 & ND & ND & 0.624 & 0.6232 & 0.6237 \\
\hline 106 & ND & ND & 0.624 & 0.6228 & 0.6232 \\
\hline 109 & ND & ND & 0.624 & 0.6228 & 0.6232 \\
\hline 112 & ND & ND & 0.625 & 0.6222 & 0.6235 \\
\hline 126 & ND & ND & 0.499 & 0.4975 & 0.4978 \\
\hline 144 & ND & ND & 0.500 & 0.4974 & 0.4982 \\
\hline 145 & ND & ND & 0.499 & 0.4976 & 0.4981 \\
\hline 155 & 0.625 & 0.624 & 0.623 & 0.6227 & 0.6227 \\
\hline 158 & ND & ND & 0.623 & 0.6230 & 0.6233 \\
\hline 161 & $\mathrm{ND}$ & ND & 0.623 & 0.6229 & 0.6236 \\
\hline 164 & ND & ND & 0.624 & 0.6224 & 0.6232 \\
\hline 167 & ND & ND & 0.624 & 0.6229 & 0.6229 \\
\hline 170 & 0.625 & 0.624 & 0.624 & 0.6229 & 0.6225 \\
\hline 180 & ND & ND & 0.500 & 0.4970 & 0.4976 \\
\hline 181 & ND & ND & 0.499 & 0.4976 & 0.4980 \\
\hline 199 & ND & ND & 0.499 & 0.4973 & 0.4979 \\
\hline 213 & ND & ND & 0.623 & 0.6232 & 0.6235 \\
\hline 216 & ND & ND & 0.624 & 0.6226 & 0.6234 \\
\hline 219 & ND & ND & 0.624 & 0.6228 & 0.6231 \\
\hline 222 & ND & ND & 0.624 & 0.6226 & 0.6229 \\
\hline 235 & ND & ND & 0.624 & 0.6228 & 0.6231 \\
\hline 244 & ND & ND & 0.624 & 0.6225 & 0.6222 \\
\hline 259 & ND & ND & 0.624 & 0.6232 & 0.6227 \\
\hline 264 & ND & ND & 0.623 & 0.6228 & 0.6231 \\
\hline 267 & ND & ND & 0.622 & 0.6218 & 0.6232 \\
\hline 270 & ND & ND & 0.623 & 0.6222 & 0.6230 \\
\hline 295 & ND & ND & 0.625 & 0.6227 & 0.6228 \\
\hline 303 & 0.625 & 0.624 & 0.623 & 0.6224 & 0.6225 \\
\hline 312 & 0.625 & 0.624 & 0.624 & 0.6227 & 0.6232 \\
\hline 319 & ND & ND & 0.622 & 0.6222 & 0.6256 \\
\hline $\operatorname{Mean}(c)$ & 0.625 & 0.6243 & 0.6242 & 0.6227 & 0.6230 \\
\hline Std. Dev. & 0 & 0.0006 & 0.0012 & 0.0002 & 0.0004 \\
\hline Mean (d) & & & 0.6239 & 0.6227 & 0.0233 \\
\hline Std. Dev. & & & 0.0009 & 0.0003 & 0.0001 \\
\hline
\end{tabular}

(a) 1 in. $=25.4 \mathrm{~mm}$.

(b) $\mathrm{ND}=$ not determined.

(c) $\mathbf{n}=6$.

${ }^{(d)} n=34$. 
TABLE 4-11

ACCURACY OF METROLOGY ROBOT MEASUREMENTS

\begin{tabular}{|c|c|c|c|c|c|c|}
\hline \multirow{2}{*}{$\begin{array}{c}\text { Type } \\
\text { of } \\
\text { Measurement }\end{array}$} & \multicolumn{3}{|c|}{ Robot vs PIE(a) } & \multicolumn{3}{|c|}{ Robot v8 $Q C^{(b)}$} \\
\hline & $\begin{array}{l}\text { Number of } \\
\text { Comparisons }\end{array}$ & $\begin{array}{l}\text { Accuracy, } 1 \sigma \\
(\text { (n. })(c)\end{array}$ & $\begin{array}{c}\text { Bias } \pm 1 \sigma(d) \\
(\ln .)(c)\end{array}$ & $\begin{array}{c}\text { Number of } \\
\text { Comparisons }\end{array}$ & $\begin{array}{l}\text { Accuracy, } \\
\left.\text { (In.) }^{10}\right)^{1 \sigma}\end{array}$ & $\begin{array}{l}\text { Bias }(e, f) \\
(\text { In.) }(c)\end{array}$ \\
\hline Fuel-element length & 42 & \pm 0.004 & $0.011 \pm 0.001$ & 324 & \pm 0.005 & 0.007 \\
\hline Distance between fiducial holes & 18 & \pm 0.007 & $0.000 \pm 0.002$ & 90 & \pm 0.003 & 0.000 \\
\hline Distance between coolant holes & 30 & \pm 0.007 & $0.002 \pm 0.001$ & -- & $\mathrm{ND}(\mathrm{g})$ & ND \\
\hline D1stance across flats & 15 & \pm 0.003 & $0.000 \pm 0.001$ & 102 & \pm 0.003 & 0.000 \\
\hline Coolant hole diameters & 40 & \pm 0.001 & $-0.001 \pm 0.000$ & -- & ND & ND \\
\hline Side face bow & 270 & \pm 0.001 & $0.000 \pm 0.000$ & -- & ND & ND \\
\hline
\end{tabular}

(a) Comparison of robot and hot cell measurements for survelllance element 1-0743.

(b) Comparison of robot and QC measurements for calibration element 8-0182.

(c) 1 in. $=25.4 \mathrm{~mm}$.

(d) ${ }_{\text {B1as }}=$ PIE-Robot.

(e) ${ }_{\text {B1as }}=$ QC-Robot.

(f) Uncertainty on blas is less than \pm 0.0005 .

(g) $\mathrm{ND}=$ not determined. 
TABLE 4-12

CALCULATED AND MEASURED IRRADIATION-INDUCED STRAINS

AND BOW FOR FSV FUEL ELEMENT 1-0743

\begin{tabular}{|c|c|c|c|}
\hline \multirow[b]{2}{*}{ Parameter } & \multicolumn{2}{|c|}{ Measured } & \multirow[b]{2}{*}{ Calculated (b) } \\
\hline & Metrology Robot & Hot $\operatorname{Ce11}(a)$ & \\
\hline $\begin{array}{l}\text { Element average } \\
\text { axial strain } \\
(\%)\end{array}$ & $-0.182 \pm 0.014$ & -0.170 & -0.158 \\
\hline $\begin{array}{l}\text { Axial strain } \\
\text { Distribution } \\
(\%)\end{array}$ & & & \\
\hline Corner 1 & -0.239 & -0.220 & -0.145 \\
\hline Corner 2 & -0.244 & -0.218 & -0.148 \\
\hline Corner 3 & -0.205 & -0.170 & -0.160 \\
\hline Corner 4 & -0.129 & -0.118 & -0.169 \\
\hline Corner 5 & -0.127 & -0.114 & -0.166 \\
\hline Corner 6 & -0.189 & -0.170 & -0.153 \\
\hline $\begin{array}{l}\text { Element average }(c) \\
\text { radial strain } \\
(\%)\end{array}$ & $-0.103 \pm 0.042$ & -0.130 & -0.075 \\
\hline Bow (mm) & 0.30 & 0.28 & 0.05 \\
\hline
\end{tabular}

(a) No error estimates made.

(b) Obtained from SURVEY/STRESS calculations based on irradiation conditions from SURVEY analysis of FSV cycle 1 (36-time-interval SURVEY based on results from detailed GAUGE analysis of FSV cycle 1).

(c) Actually, the average radial strain at the top of the element. 
TABLE 4-13

PLENUM DEPTH, FUEL STACK LENGTH, AND PUSH-OUT FORCE MEASUREIENTS FOR FSV FUEL ELEMENT 1-0743

\begin{tabular}{|c|c|c|c|c|c|c|c|c|}
\hline \multirow[b]{2}{*}{ Hole } & \multicolumn{3}{|c|}{ Plenum Depth (in.) (a) } & \multicolumn{3}{|c|}{ Stack Length (in.) (a) } & \multicolumn{2}{|c|}{$\begin{array}{l}\text { Push-Out } \\
\text { Force (1b) (a) }\end{array}$} \\
\hline & Pre I & PIE & $\begin{array}{l}\text { PIE- } \\
\text { Pre I }\end{array}$ & Pre I & PIE & PIE-Pre I & Initial & Sustaining \\
\hline 12 & 1.630 & 1.7290 & +0.0990 & 29.140 & 29.0216 & -0.1184 & 0 & 0 \\
\hline 47 & 2.453 & 2.5619 & +0.1089 & 27.177 & 27.1108 & -0.0662 & 0 & 4 \\
\hline 157 & 1.649 & 1.7772 & +0.1282 & 29.121 & (b) & (b) & $2.5^{(\mathrm{b})}$ & 1 \\
\hline 189 & 1.645 & 1.7534 & +0.1084 & 29.125 & 29.0206 & -0.1044 & 0 & 2 \\
\hline 278 & 1.654 & 1.7647 & +0.1107 & 29.116 & 29.0129 & -0.1031 & 1 & 1 \\
\hline 285 & 1.661 & 1.7965 & +0.1355 & 29.109 & 28.9455 & -0.1635 & 0 & 3 \\
\hline Avg. & 1.782 & 1.8971 & +0.1151 & 28.798 & 28.6223 & -0.1111 & 0.58 & 1.83 \\
\hline Std. Dev. & 0.329 & 0.3265 & +0.0138 & 0.7942 & 0.8455 & 0.0351 & 1.02 & 1.47 \\
\hline 86 & -- & - & - & -- & -- & -- & 1.5 & 1 \\
\hline 121 & -- & -- & -- & -- & -- & -- & 1 & 1 \\
\hline 160 & -- & -- & -- & -- & -- & -- & 18 & 5 \\
\hline 194 & -- & - & -- & -- & -- & -- & 2 & 1 \\
\hline 231 & - & -- & -- & -- & -- & -- & 22 & 1 \\
\hline Avg. & -- & -- & -- & -- & -- & -- & 8.90 & 1.80 \\
\hline Std. Dev. & -- & -- & -- & -- & -- & -- & 10.24 & 1.79 \\
\hline
\end{tabular}

(a) 1 in. $=25.4 \mathrm{~mm} ; 11 \mathrm{~b}=4.448 \mathrm{~N}$.

(b) 1115 rods broken in stack during unloading. 
TABLE $4-14$

BROKEN FUEL PARTICLES OBSERVED ON SURFACES OF SEVENTEEN FUEL RODS FROM FSV FUEL ELEMENT 1-0743

\begin{tabular}{|c|c|}
\hline Rod ID & $\begin{array}{c}\text { No. of Broken } \\
\text { Particles }\end{array}$ \\
\hline $12-2$ & 16 \\
\hline $12-7$ & 9 \\
\hline $12-13$ & 12 \\
\hline $47-2$ & 14 \\
\hline $47-7$ & 16 \\
\hline $47-8$ & 9 \\
\hline $47-14$ & 8 \\
\hline $189-2$ & 21 \\
\hline $189-7$ & 10 \\
\hline $189-14$ & 15 \\
\hline $278-2$ & 9 \\
\hline $278-8$ & 21 \\
\hline $278-13$ & 17 \\
\hline $285-2$ & 9 \\
\hline $285-7$ & 9 \\
\hline $285-8$ & 11 \\
\hline $285-13$ & 11 \\
\hline Tota1 & $\overline{217}$ \\
\hline Mean & 13 \\
\hline
\end{tabular}


TABLE 4-15

MEASURED STRAINS FOR FUEL RODS

IRRADIATED IN FSV FUEL ELEMENT 1-0743

\begin{tabular}{|c|c|c|c|c|c|c|}
\hline \multirow{3}{*}{$\begin{array}{l}\text { Fue } 1 \\
\text { Stack } \\
\text { ID (a) }\end{array}$} & \multirow{3}{*}{$\begin{array}{l}\text { Time and } \\
\text { Fuel Stack } \\
\text { Averaged } \\
\text { Temperature } \\
\left({ }^{\circ} \mathrm{C}\right)\end{array}$} & \multirow{3}{*}{$\begin{array}{c}\text { Stack Averaged } \\
\text { Fast } \\
\text { Fluence } \\
\left(10^{25} \mathrm{n} / \mathrm{m}^{2}\right) \\
(\mathrm{E}>29 \mathrm{fJ})_{\text {HTGR }}\end{array}$} & \multicolumn{4}{|c|}{ Stack Averaged Fuel Rod Strain } \\
\hline & & & \multicolumn{2}{|c|}{ Radia1 } & \multicolumn{2}{|c|}{ Axia1 } \\
\hline & & & $\begin{array}{c}\text { Strain } \\
(\%)\end{array}$ & $\begin{array}{l} \pm 1 \sigma \\
(\%)\end{array}$ & $\begin{array}{c}\text { Strain } \\
(\%)\end{array}$ & $\begin{array}{l} \pm 1 \sigma \\
(\%)\end{array}$ \\
\hline 12 & 645 & 0.84 & -0.31 & 0.05 & -0.47 & 0.06 \\
\hline 47 & 645 & 0.83 & -0.34 & 0.02 & -0.44 & 0.03 \\
\hline 189 & 675 & 1.00 & -0.34 & 0.02 & -0.47 & 0.03 \\
\hline 278 & 690 & 1.10 & -0.43 & 0.02 & -0.50 & 0.04 \\
\hline 285 & 695 & 1.10 & -0.39 & 0.05 & -0.59 & 0.03 \\
\hline
\end{tabular}

(a) These fuel stacks contained only fuel rods that had been dimensionally characterized prior to irradiation. Fuel stack 157 also contained precharacterized fuel rods, but all were broken during unloading from the element. 
TABLE 4-16

DIMENSIONAL AND STRAIN DATA FOR FUEL RODS IRRADIATED IN FUEL STACK 12 OF FSV FUEL ELEMENT 1-0743

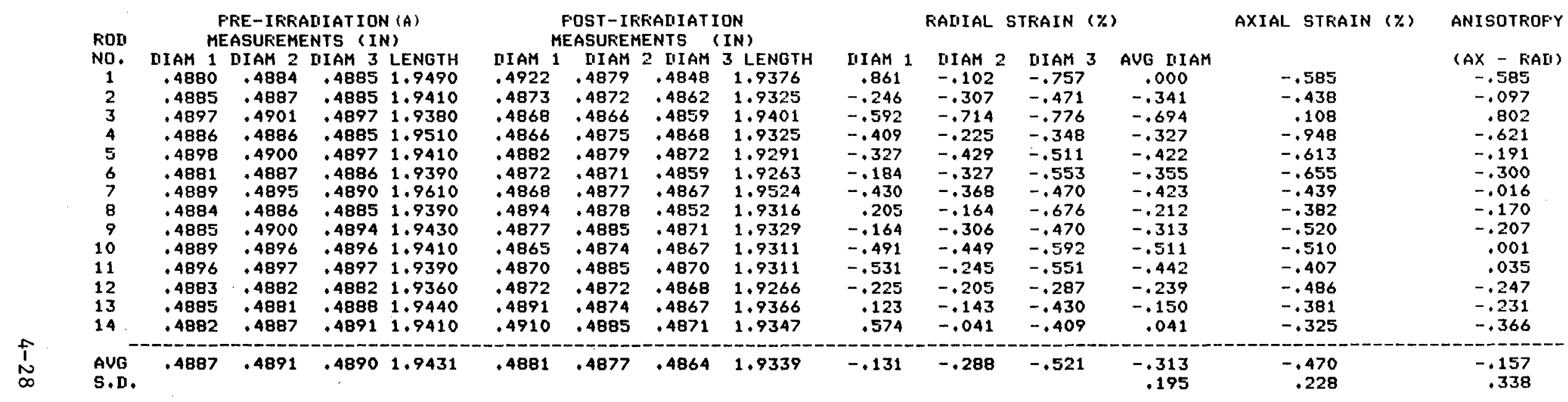

(A) PRE-IRFADIATION AIR BAUGE MEASUREMENTS WERE INCREASE! BY 0.0014 INCH TO MAKE MEASUREMENTS COMPATIRLE WITH THE FOST-IRRADIATION MICROMETEK TYFE MEASUREMENTS (REF. 17)

1 IN. $=25.4$ MM 
TABLE $\quad 4-17$

DIMENSIONAL AND STRAIN DATA FOR FUEL RODS IRRADIATED IN FUEL STACK 47 OF FSV FUEL ELEMENT 1-0743

\begin{tabular}{|c|c|c|c|c|c|c|c|c|c|c|c|c|c|c|}
\hline FOD & & $\begin{array}{l}\text { RE-IRRA } \\
\text { SASUREME }\end{array}$ & $\begin{array}{l}\text { ADIATION } \\
\text { ENTS (II }\end{array}$ & $\begin{array}{l}\text { V (A) } \\
\text { V) }\end{array}$ & ME & $\begin{array}{l}\text { OST-IRF } \\
\text { ASUREME }\end{array}$ & $\begin{array}{l}\text { ZADIAT } \\
\text { ETS }\end{array}$ & $\begin{array}{l}\text { ION } \\
\text { (IN) }\end{array}$ & & FALIIAL 5 & TRAIN & & AXIAL STRAIN (\%) & ANISOTFOFYY \\
\hline NO. & LIAM 1 & DIAM 2 & DIAM 3 & LENGTH & IIIAM 1 & UIAM 2 & 2 IIAM & 3 LENGTH & DIAM 1 & IIIAM 2 & DIAM 3 & AVG DIAM & & (AX - FALI) \\
\hline 1 & .4893 & .4902 & .4899 & 1.9370 & .4877 & .4880 & .4875 & 1.9310 & -.327 & -.449 & -.490 & -.422 & -.310 & .112 \\
\hline 2 & .4884 & .4879 & .4887 & 1.9380 & .4867 & .4869 & .4866 & 1.9278 & -.348 & -.205 & -.430 & -.328 & -.526 & -.199 \\
\hline 3 & .4886 & .4890 & .4889 & 1.9390 & .4879 & .4868 & .4866 & 1.9279 & -.143 & -.450 & -.470 & -.355 & -.572 & -.218 \\
\hline 4 & .4890 & .4897 & .4898 & 1.9410 & .4880 & .4877 & .4862 & 1.9297 & -.204 & -.408 & -.735 & -.449 & -.582 & -.133 \\
\hline 5 & .4881 & .4896 & .4897 & 1.9430 & .4887 & .4875 & .4866 & 1.9341 & .123 & -.429 & -.633 & -.313 & -.458 & -.145 \\
\hline 6 & .4891 & .4891 & .4890 & 1.9420 & .4867 & .4883 & .4867 & 1.9331 & -.491 & -.164 & -.470 & -.375 & -.458 & -.083 \\
\hline 7 & .4881 & .4886 & .4888 & 1.9420 & .4867 & .4876 & .4858 & 1.9314 & -.287 & -.205 & -.614 & -.368 & -.546 & -.177 \\
\hline 8 & .4889 & .4897 & .4895 & 1.9390 & .4880 & .4885 & .4866 & 1.9319 & -.184 & -.245 & -.592 & -.341 & -.366 & -.026 \\
\hline 9 & .4881 & .4887 & .4887 & 1.9420 & .4877 & .4865 & .4866 & 1.9340 & -.082 & -.450 & -.430 & -.321 & -.412 & -.091 \\
\hline 10 & .4889 & .4891 & .4894 & 1.9400 & .4871 & .4873 & .4866 & 1.9349 & -.368 & -.368 & -.572 & -.436 & -.263 & .173 \\
\hline 11 & .4882 & .4885 & .4887 & 1.9380 & .4875 & .4877 & .4871 & 1.9299 & -.143 & -.164 & -.327 & -.212 & -.418 & -.206 \\
\hline 12 & .4891 & .4896 & .4896 & 1.9570 & .4887 & .4880 & .4868 & 1.9462 & -.082 & -.327 & -.572 & -.327 & -.552 & -.225 \\
\hline 13 & .4886 & .4888 & .4887 & 1.9400 & .4871 & .4873 & .4875 & 1.9330 & -.307 & -.307 & -.246 & -.286 & -.361 & -.074 \\
\hline 14 & .4884 & .4894 & .4896 & 1.9390 & .4881 & .4875 & .4882 & 1.9339 & -.061 & -.388 & -.286 & -.245 & -.263 & -.018 \\
\hline & .4886 & .4891 & .4892 & 1.9412 & .4876 & .4875 & .4868 & 1.9328 & -.208 & -.326 & -.491 & -.341 & -.435 & -.094 \\
\hline S.n. & .4000 & .4072 & .4072 & 107412 & .4010 & .4010 & .4000 & $1,70<0$ & -.200 & $-.3<0$ & -.471 & .068 & .112 & .122 \\
\hline
\end{tabular}

(A) FRE-IRRALIATION AIR GAUGE MEASUREMENTS WERE INCREASED EY 0.0014 INCH TD MAKE MEASUREMENTS COMF'ATIELE WITH THE FOST-IRRADIATION MICROMETER TYFE MEASUREMENTS (REF. 17)

$1 \mathrm{IN}=25.4 \mathrm{~m}$ 
TABLE $4-18$

DIMENSIONAL AND STRAIN DATA FOR FUEL RODS IRRADIATED IN FUEL STACK 189 OF FSV FUEL ELEMENT 1-0743

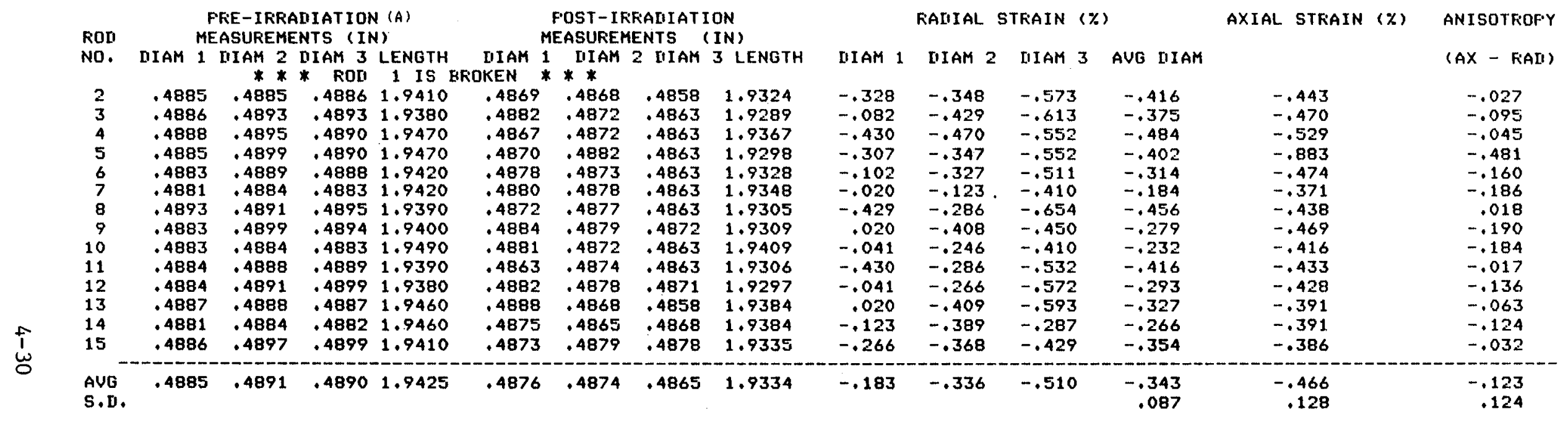

(A) FRE-IRRADIATION AIR GAUGE MEASUREMENTS WERE INCREASED BY 0.0014 INCH TO MAKE MEASUFEMENTS COMPATIELE WITH THE FOST-IRRADIATION MICROMETER TYFE MEASUREMENTS (REF. 17)

$1 \mathrm{NN}=25.4 \mathrm{MM}$ 
TABLE $4-19$

DIMENSIONAL AND STRAIN DATA FOR FUEL RODS IRRADIATED IN FUEL STACK 278 OF FSV FUEL ELEMENT 1-0743

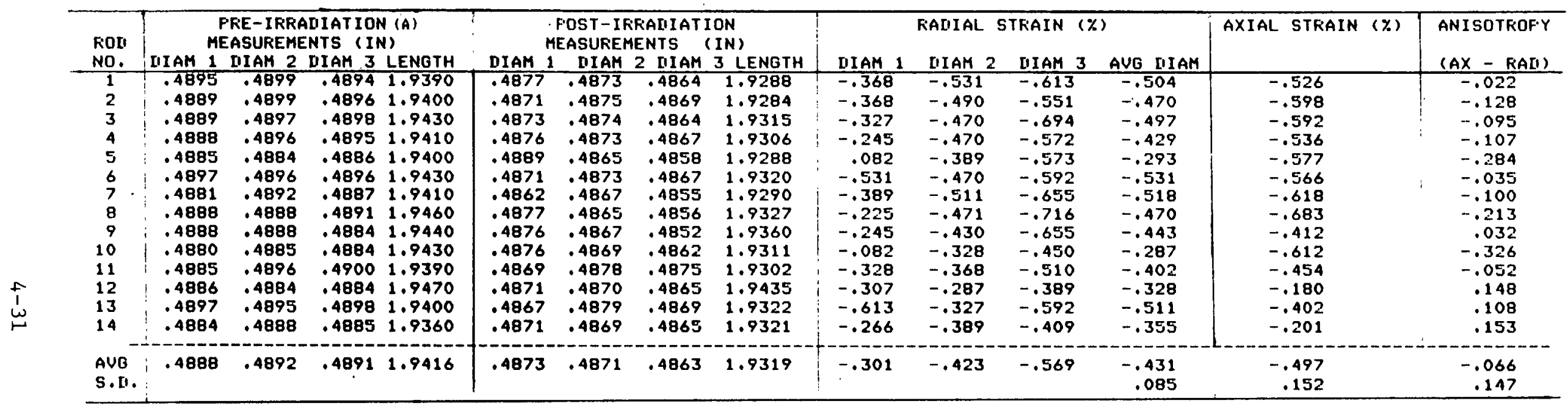

(A) PRE-IRRAIIATION AIR GAUGE MEASUREMENTS WERE INCREASEI

EY 0.0014 INCH TO MAKE MEASUREMENTS COMFATI ILE WITH THE

FOST-IRRADIATION MICROMETER TYFE MEASUREMENTS (REF. 17)

1 IN. $=25.4$ MM 
TABLE $4-20$

DIMENSIONAL AND STRAIN DATA FOR FUEL RODS IRRADIATED IN FUEL STACK 285 OF FSV FUEL ELEMENT 1-0743

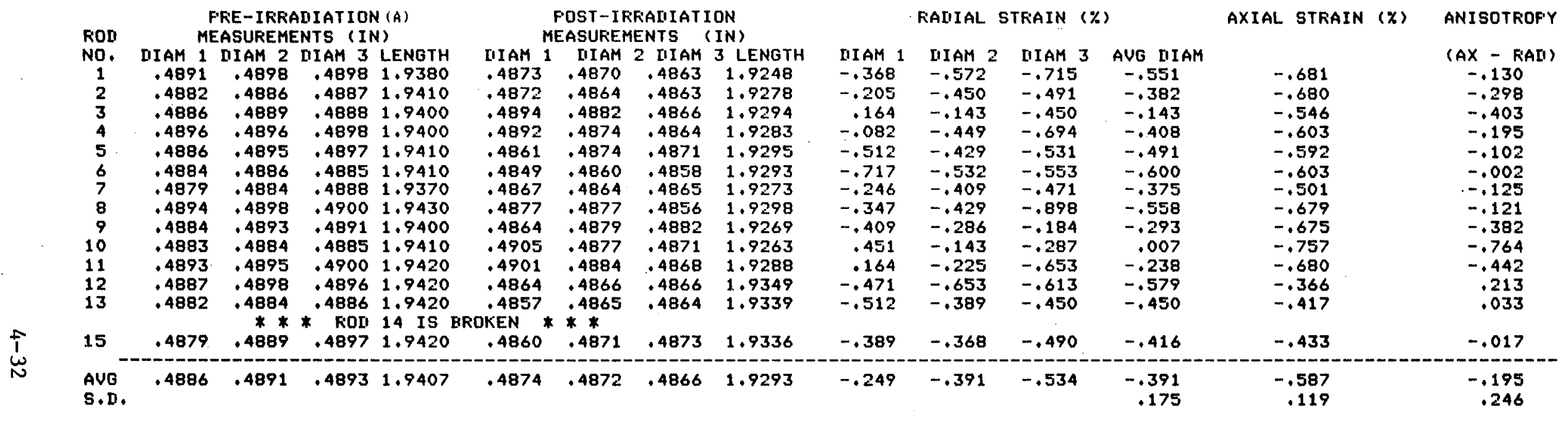

(A) FRE-IRRAIIATION AIF GAUGE MEASUREMENTS WERE INCREASEI BY 0.0014 INCH TO MAKE MEASUREMENTS COMFATIBLE WITH THE FOST-IRRAIIIATION MICFOMETER TYFE MEASUFEMENTS (REF. 17

$1 \mathrm{IN}=25.4 \mathrm{NM}$ 
TABLE 4-21

COMPRESSION TESTING OF FUEL RODS FROM

FSV FUEL ELEMENT 1-0743

FAILURE LOAD AT RUPTURE

\begin{tabular}{|c|c|c|c|}
\hline \multicolumn{2}{|c|}{ Unirradiated Rods } & \multicolumn{2}{|c|}{ Irradiated Rods } \\
\hline $\begin{array}{l}\text { Fuel Rod } \\
\text { ID }\end{array}$ & $\begin{array}{l}\text { Force } \\
(1 b)(a)\end{array}$ & $\begin{array}{l}\text { Fuel Rod } \\
\text { ID }\end{array}$ & $\begin{array}{l}\text { Force } \\
\text { (1b) (a) }\end{array}$ \\
\hline 1 & 113 & $70-1$ & 125 \\
\hline 2 & 95 & $70-2$ & 155 \\
\hline 3 & 100 & $70-3$ & 139 \\
\hline 4 & 110 & $70-4$ & 136 \\
\hline 5 & 95 & $70-5$ & 118 \\
\hline 6 & 114 & $70-6$ & 121 \\
\hline 7 & 102 & $70-7$ & 123 \\
\hline 8 & 103 & $70-8$ & 151 \\
\hline 9 & 124 & $70-9$ & 127 \\
\hline \multirow[t]{6}{*}{10} & 102 & $70-10$ & 100 \\
\hline & & $70-11$ & 122 \\
\hline & & $70-12$ & 102 \\
\hline & & $70-13$ & 125 \\
\hline & & $70-14$ & 124 \\
\hline & & $70-15$ & 110 \\
\hline Mean & 105.8 & & 121.8 \\
\hline Standard deviation & 9.3 & & 13.3 \\
\hline Standard deviation $/ \sqrt{n}$ & 2.9 & & 3.7 \\
\hline
\end{tabular}

(a) $11 b=4.448 \mathrm{~N}$ 
TABLE 4-22

FISSION GAS RELEASE MEASUREMENTS FOR FUEL RODS IRRADIATED IN FSV FUEL ELEMENT 1-0743

\begin{tabular}{|c|c|c|c|c|c|c|}
\hline \multirow{2}{*}{$\begin{array}{l}\text { Fuel Rod } \\
\text { ID }\end{array}$} & \multirow{2}{*}{$\begin{array}{c}\text { Time- } \\
\text { Averaged } \\
\text { Maximum Fuel } \\
\text { Temp } \\
\left({ }^{\circ} \mathrm{C}\right)(\mathrm{a})\end{array}$} & \multirow{2}{*}{$\begin{array}{c}\text { Fast }(a) \\
\text { F1uence } \\
\left(10^{25} \mathrm{n} / \mathrm{m} 2\right) \\
(\mathrm{E}>29 \mathrm{fJ})_{\mathrm{HTGR}}\end{array}$} & \multicolumn{2}{|c|}{ Fission Gas Release(b) } & \multicolumn{2}{|c|}{$\begin{array}{r}\text { Burnup (a) } \\
(\% \text { FIMA) }\end{array}$} \\
\hline & & & Preirrad. & Postirrad. & F1ssile & Fertile \\
\hline $12-2$ & 690 & 0.8 & & $1.1 \times 10^{-4}$ & 6.1 & 0.3 \\
\hline $12-7$ & 660 & 0.8 & & & 6.2 & \\
\hline $12-13$ & 625 & 0.8 & & & 6.2 & \\
\hline $47-2$ & 685 & 0.8 & & & 6.1 & \\
\hline $47-7$ & 660 & 0.8 & & & 6.2 & \\
\hline $47-14$ & 625 & $0.8\}$ & & & 6.2 & \\
\hline $189-7$ & 695 & 1.0 & & & 6.2 & \\
\hline $285-2$ & 750 & 1.1 & & & 6.1 & \\
\hline $285-7$ & 720 & 1.1 & & & 6.2 & \\
\hline $285-14$ & 680 & 1.1 & & & 6.2 & \\
\hline $47-8$ & 655 & 0.8 & & $\downarrow$ & 6.2 & \\
\hline $278-8$ & 745 & 1.0 & $\left.1.3 \times 10^{-4(c)}\right\}$ & $9.3 \times 10^{-5}$ & 6.2 & \\
\hline $285-8$ & 710 & 1.1 & & & 6.2 & \\
\hline $189-2$ & 720 & 1.0 & & $9.2 \times 10^{-5}$ & 6.1 & \\
\hline $189-14$ & 655 & 1.0 & & $5.5 \times 10^{-5}$ & 6.2 & \\
\hline $278-12$ & 745 & 1.0 & & $8.2 \times 10^{-5}$ & 6.2 & \\
\hline $278-13$ & 670 & 1.1 & & $8.8 \times 10^{-5}$ & 6.2 & \\
\hline Average & 690 & 0.9 & $1.3 \times 10^{-4}$ & $1.0 \times 10^{-4}$ & 6.2 & 0.3 \\
\hline
\end{tabular}

(a) From SURVEY analysis based on detailed (335 time intervals) GAUGE analysis of cycle 1 and axial power and flux profiles from FEVER.

(b) $\mathrm{R} / \mathrm{B}$ of $\mathrm{Kr}-85 \mathrm{~m}$ at $1000^{\circ} \mathrm{C}$.

(c) Measured on group of five rods including rods 47-8, 278-8, and 285-8. Rod 157-8, one of the five rods, was broken during disassembly and could not be measured for fission gas release. 
TABLE $\quad 4-23$

FISSILE PARTICLE RESULTS OF METALLOGRAPHIC EXAMINATION OF FUEL RODS IRRADIATED IN FSV FUEL ELEMENT 1-0743

\begin{tabular}{|c|c|c|c|c|c|c|c|c|c|c|}
\hline \multirow{4}{*}{$\begin{array}{c}\text { Fuel Rod } \\
\text { ID }\end{array}$} & \multicolumn{3}{|c|}{ Irradiation Conditions } & & . & & & & & \\
\hline & \multirow{3}{*}{$\begin{array}{l}\text { Maximum } \\
\text { Time- } \\
\text { Averaged } \\
\text { Temp. } \\
\left({ }^{\circ} \mathrm{C}\right)\end{array}$} & \multirow{3}{*}{$\begin{array}{c}\text { Fluence } \\
\text { x } 10^{25} \mathrm{n} / \mathrm{m}^{2} \\
(\mathrm{E}>29 \mathrm{fJ})_{\mathrm{HTGR}}\end{array}$} & \multirow[b]{3}{*}{$\begin{array}{l}\text { Burnup } \\
\text { (\% FIMA) }\end{array}$} & \multicolumn{7}{|c|}{ Fissile Particles } \\
\hline & & & & \multicolumn{6}{|c|}{ Faflure $(\%)$} & \multirow{2}{*}{$\begin{array}{c}\text { IPyC } \\
\text { Debonding } \\
(\%)\end{array}$} \\
\hline & & & & $\begin{array}{l}\text { Particles } \\
\text { Examined }\end{array}$ & Buffer & IPyC & $\mathrm{S} 1 \mathrm{C}$ & OPyC & $\begin{array}{l}\text { Total } \\
\text { Coating }\end{array}$ & \\
\hline $189-2$ & 720 & 1.0 & 6.1 & 316 & 0 & 0 & 0. & 0 & 0 & 11.1 \\
\hline $189-14$ & 655 & 1.0 & 6.2 & 337 & 0 & 1.8 & 1.5 & 0.6 & 0.6 & 17.5 \\
\hline $278-2$ & 745 & 1.0 & 6.1 & 333 & 0 & 0.3 & 1.2 & 1.2 & 0.3 & 14.4 \\
\hline $278-8$ & 705 & 1.1 & 6.2 & 521 & 0 & 0.2 & 0.4 & 0.2 & 0.2 & 11.1 \\
\hline $\begin{array}{l}\text { Average } \\
95 \% \text { confidence }\end{array}$ & 705 & 1.0 & 6.2 & $\begin{array}{l}1507 \\
\text { (total) }\end{array}$ & 0 & $0.3 \leq \mathrm{F} \leq 0.9$ & $0.4 \leq \mathrm{F} \leq 1.2$ & $0.2 \leq{ }^{0.5} \leq 0.8$ & $0.1 \leq F \leq 0.5$ & 15.0 \\
\hline
\end{tabular}


TABLE 4-24

FERTILE ANALYSIS RESULTS OF METALLOGRAPHIC EXAMINATION OF FUEL RODS IRRADIATED IN FSV FUEL ELEMENT 1-0743

\begin{tabular}{|c|c|c|c|c|c|c|c|c|c|c|c|}
\hline \multirow[b]{4}{*}{$\begin{array}{l}\text { Fuel Rod } \\
\text { ID }\end{array}$} & \multicolumn{3}{|c|}{ Irradiation Conditions } & & & & & & & \multirow{4}{*}{$\begin{array}{c}\text { IPyC } \\
\text { Debonding } \\
(\%)\end{array}$} & \multirow{4}{*}{$\begin{array}{c}\text { Matrix } \\
\text { Macro- } \\
\text { porosity } \\
(\%)\end{array}$} \\
\hline & \multirow{3}{*}{$\begin{array}{l}\text { Maximum } \\
\text { Time- } \\
\text { Averaged } \\
\text { Temp. } \\
\left({ }^{\circ} \mathrm{C}\right)\end{array}$} & \multirow{3}{*}{$\begin{array}{c}\text { Fluence } \\
\text { x } 10^{25} \mathrm{n} / \mathrm{m}^{2} \\
(\mathrm{E}>29 \mathrm{fJ})_{\mathrm{HTGR}}\end{array}$} & \multirow[b]{3}{*}{$\begin{array}{l}\text { Burnup } \\
\text { (\% FIMA) }\end{array}$} & \multicolumn{6}{|c|}{ Fertile Particles } & & \\
\hline & & & & \multirow{2}{*}{$\begin{array}{l}\text { Number of } \\
\text { Particles } \\
\text { Examined }\end{array}$} & \multicolumn{5}{|c|}{ Faflure (\%) } & & \\
\hline & & & & & Buffer & IPyC & $\mathrm{Sic}$ & OPyC & $\begin{array}{c}\text { Total } \\
\text { Coating }\end{array}$ & & \\
\hline $189-2$ & 720 & 1.0 & 0.3 & 266 & $\mathrm{ND}(\mathrm{a})$ & ND & 0.8 & 1.5 & 0.4 & $\mathrm{ND}$ & 36.4 \\
\hline $189-14$ & 655 & 1.0 & 0.3 & 186 & 2.7 & 0.5 & 0 & 0 & 0 & 9.1 & 21.6 \\
\hline $278-2$ & 745 & 1.0 & 0.3 & 267 & 1.9 & 2.6 & 1.1 & 2.2 & 0.4 & 6.0 & ND \\
\hline $278-8$ & 705 & 1.1 & 0.3 & 204 & ND & ND & 0 & 0 & 0 & ND & $20.5^{-}$ \\
\hline $\begin{array}{c}\text { Average } \\
95 \% \text { confidence }\end{array}$ & 705 & 1.0 & 0.3 & $\begin{array}{c}923 \\
\text { (tota1) }\end{array}$ & $\begin{array}{l}2.2 \\
\mathrm{ND}\end{array}$ & $\begin{array}{l}1.7 \\
\text { ND }\end{array}$ & $0.2 \leq \mathrm{F} \leq 1.2$ & $0.6 \leq \mathrm{F} \leq 1.8$ & $0.0 \leq \mathrm{F} \leq 0.7$ & 7.3 & 26.2 \\
\hline
\end{tabular}

(a) $\mathrm{ND}$ - not determined. 


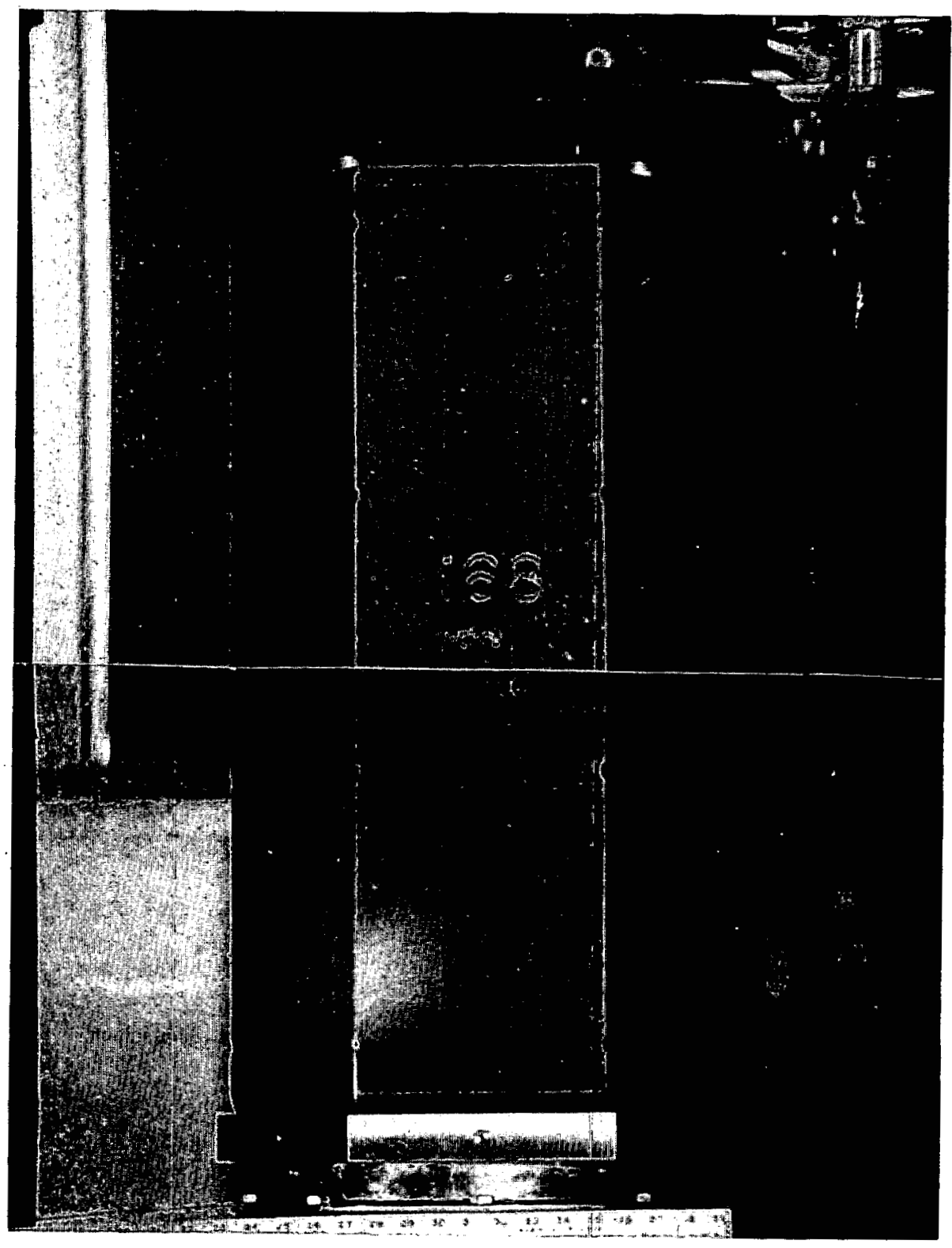

Fig. 4-1. FSV fuel element 1-0743, side face A: vertical and horizontal scrapes observed in vicinity of serial number 


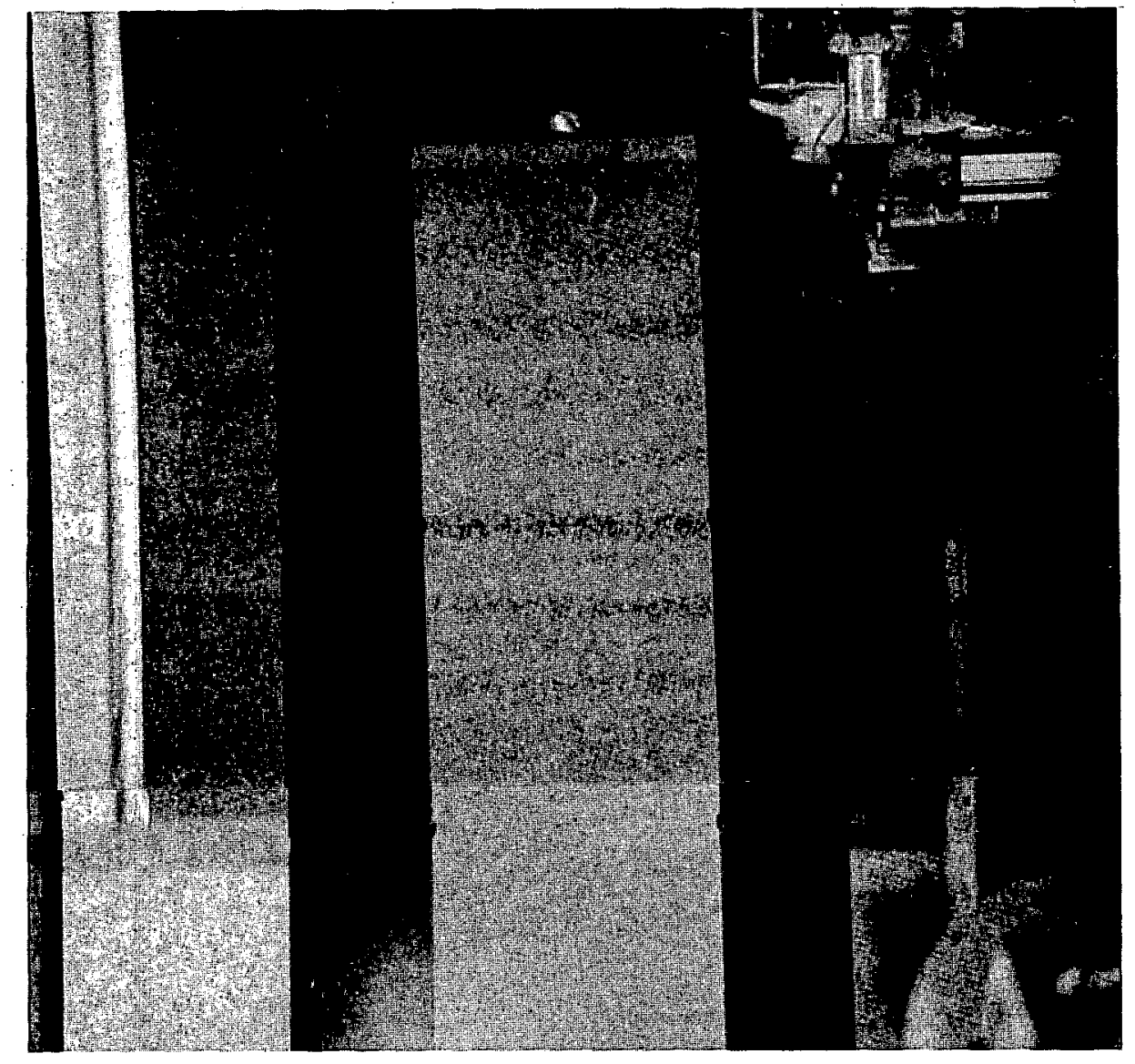

Fig. 4-2. FSV fuel element 1-0743, side face B: long scratch observed starting near top center and extending down face 


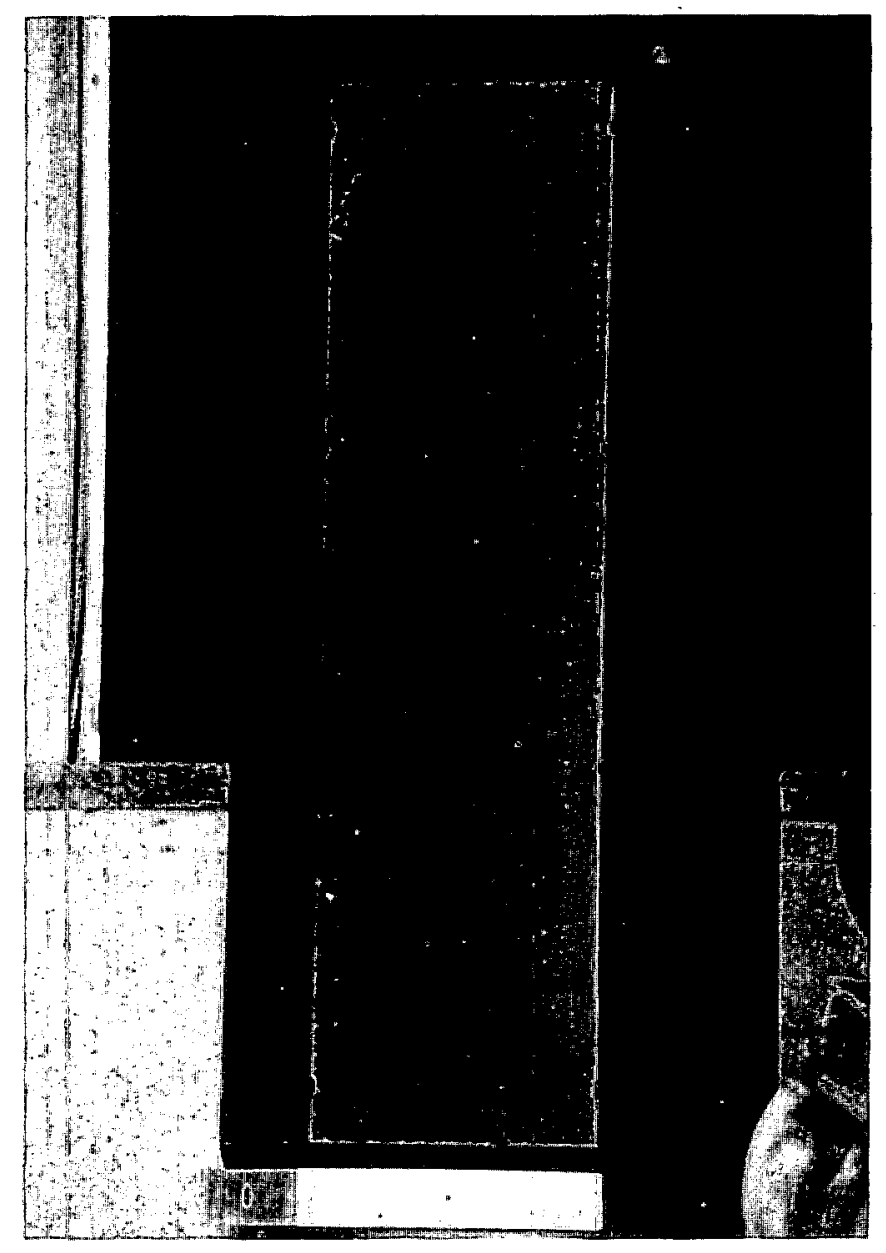

Fig. 4-3. FSV fuel element 1-0743, side face C: rub marks observed down both sides of element 


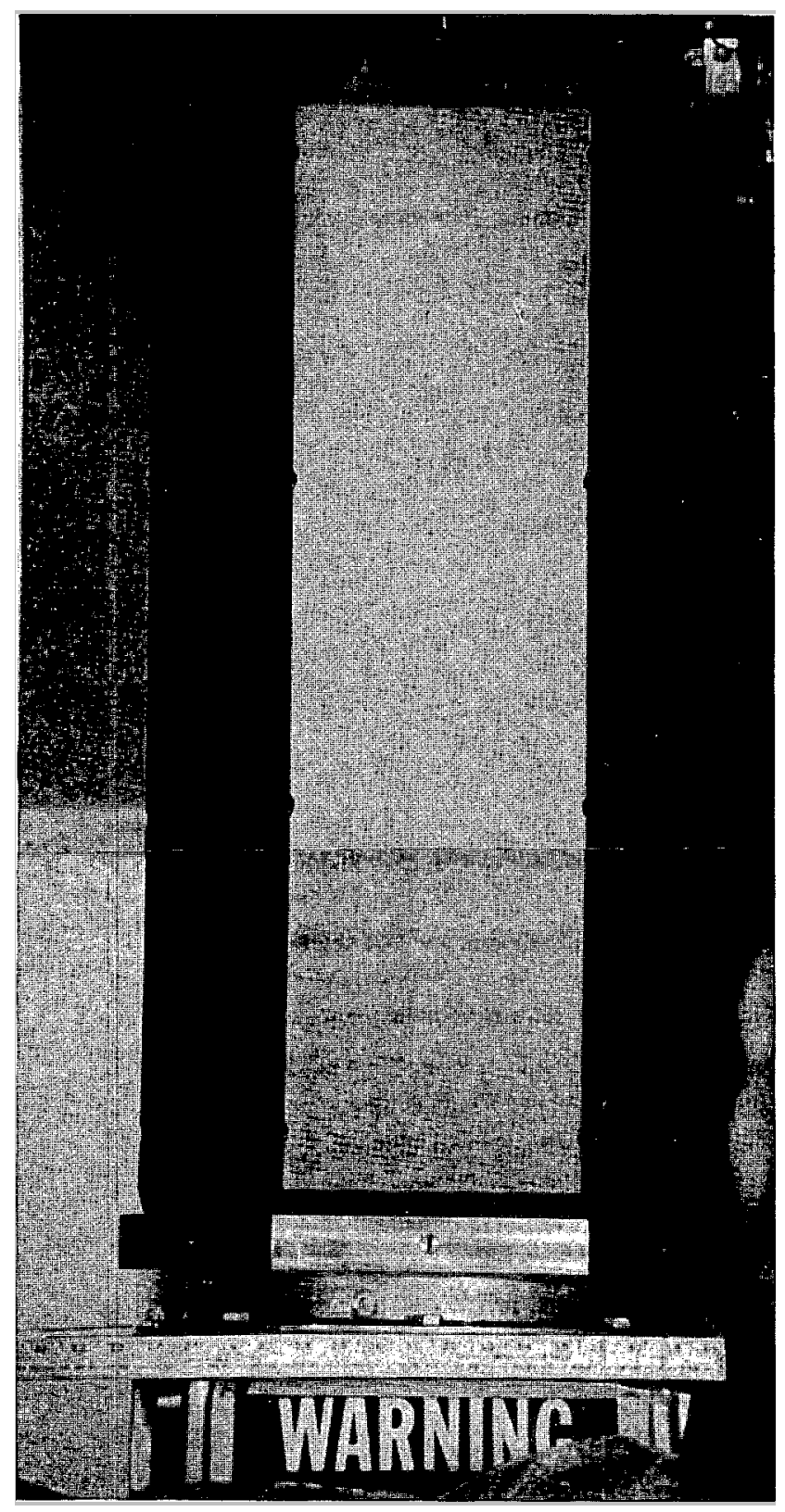

Fig. 4-4. FSV fuel element 1-0743, side face D: rub marks and scrapes observed on surface of element 


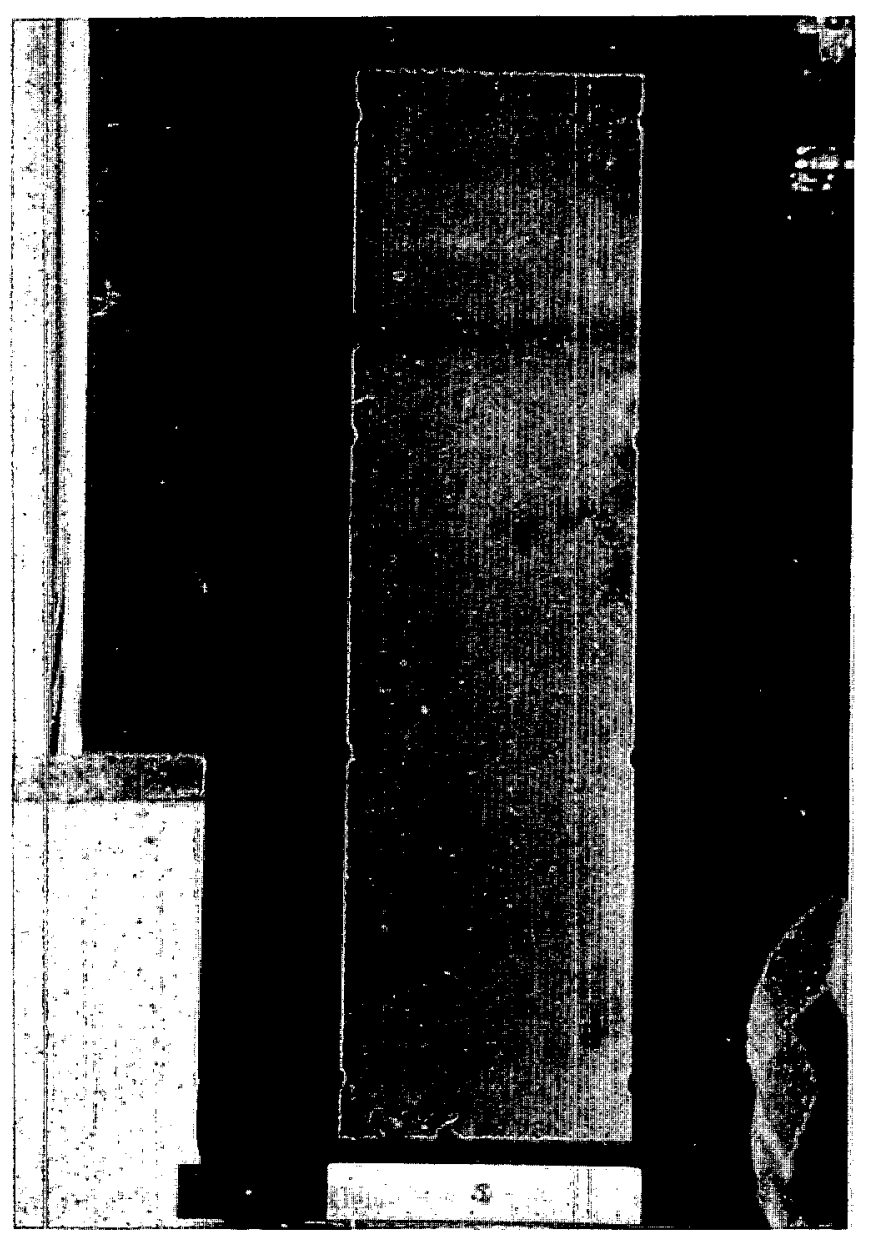

Fig. 4-5. FSV fuel element 1-0743, side face E: numerous dark smudges (most likely soot deposits) observed on surface of element 


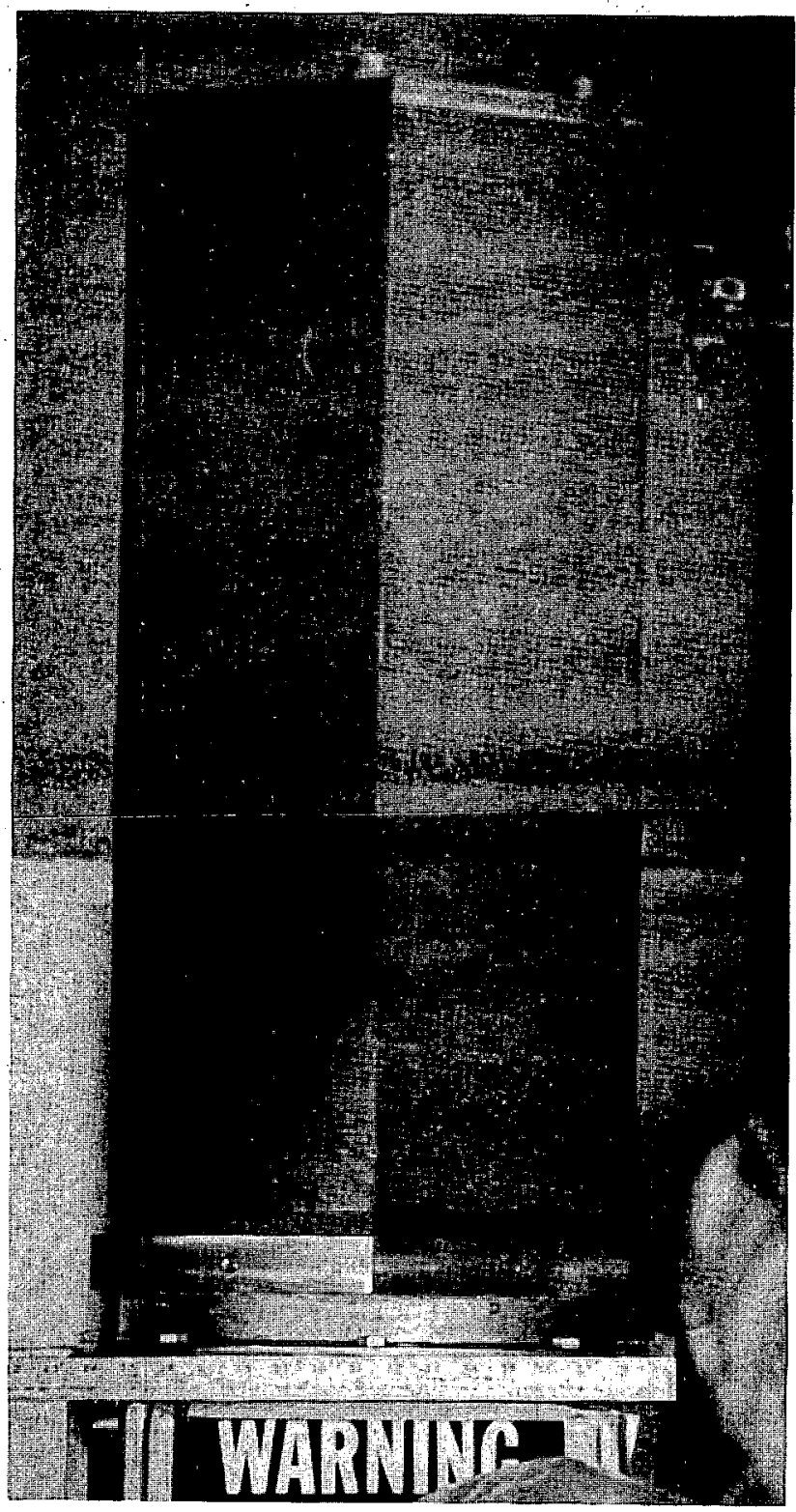

Fig. 4-6. FSV fuel element 1-0743, side faces $E$ (on left) and F (on right); numerous horizontal markings (most likely scrapes) observed on face $F$ 


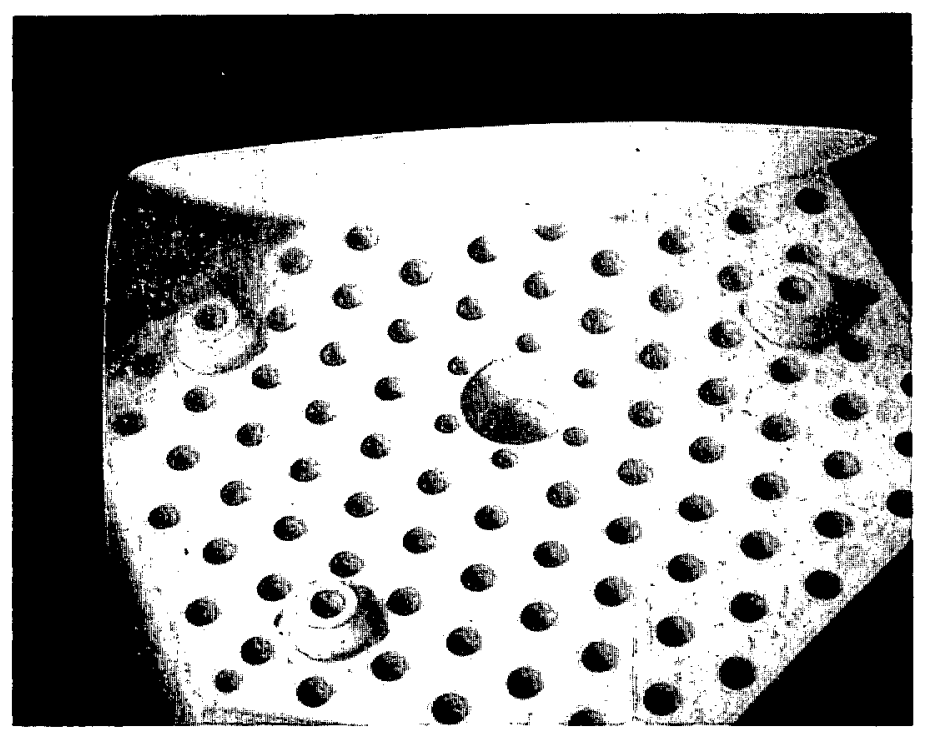

Fig. 4-7. FSV fuel element 1-0743, top surface: fuel handling machine extension sleeve is at top of photograph

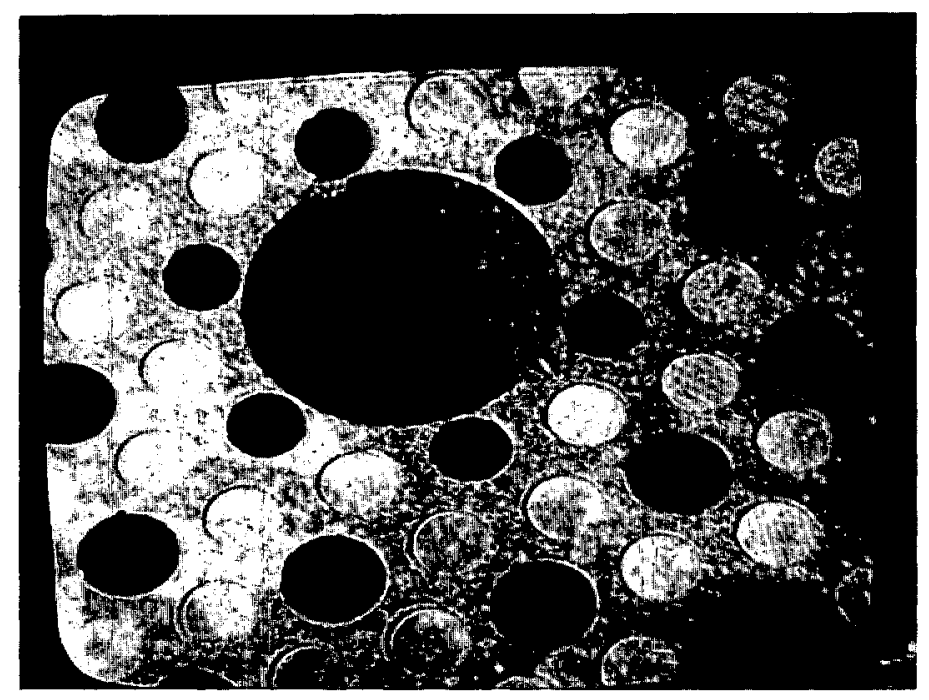

Fig. 4-8. FSV fuel element 1-0743, close-up of fuel handling hole: sma11 chip observed at edge of hole 


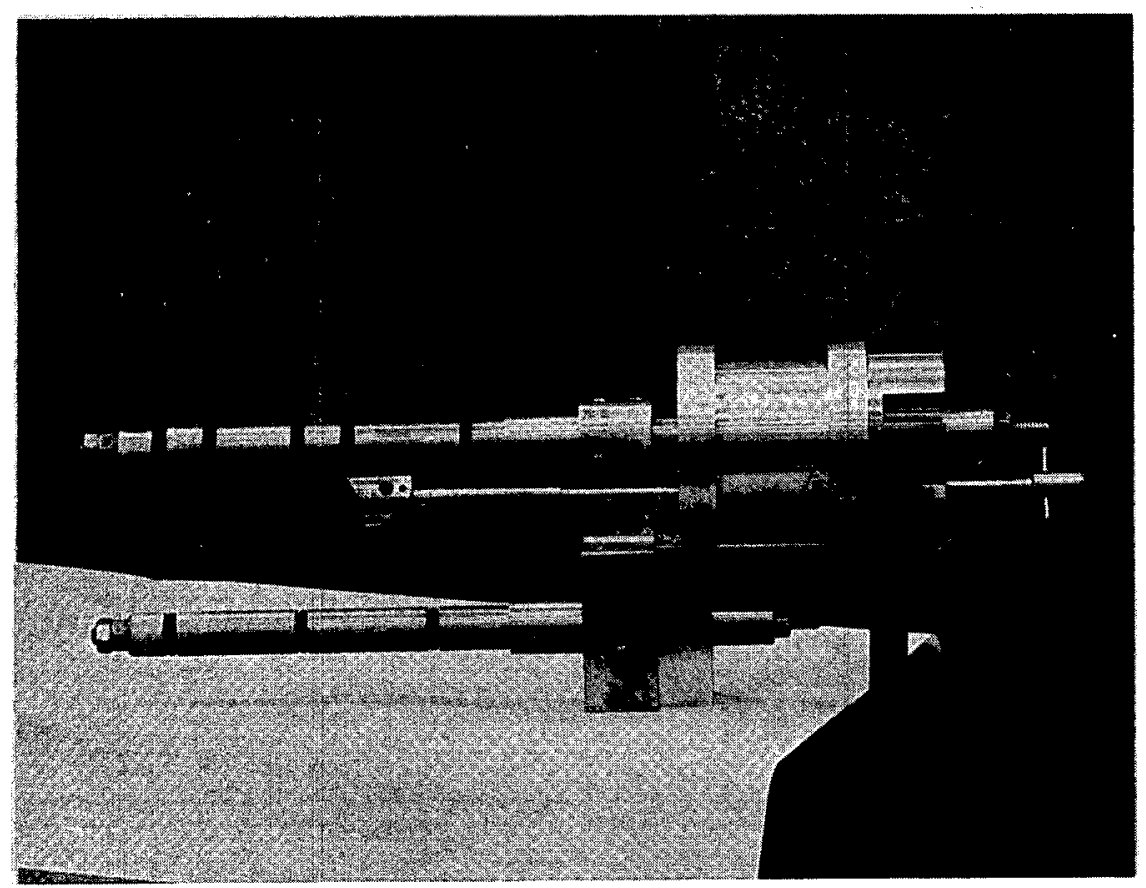

Fig. 4-9. Coring tool 


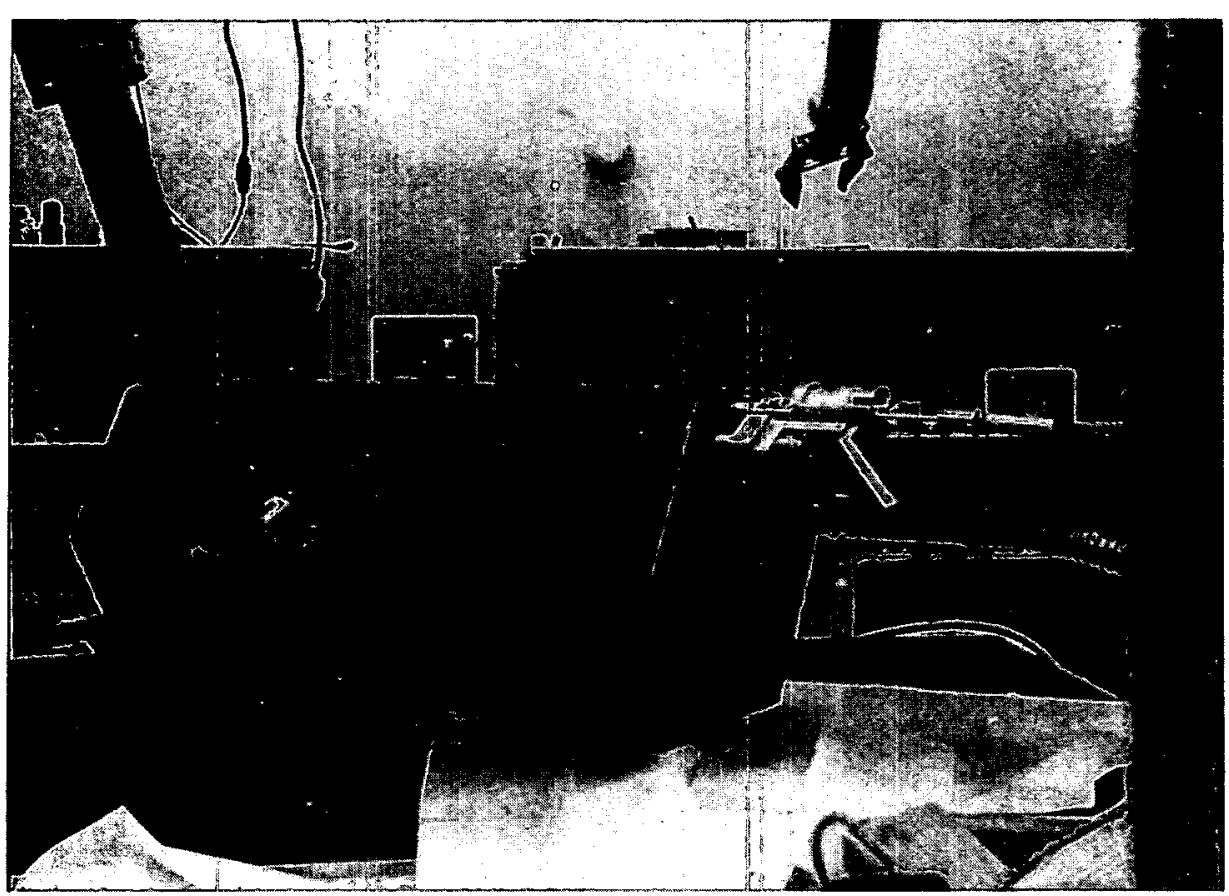

Fig. 4-10. Coring tool in operation

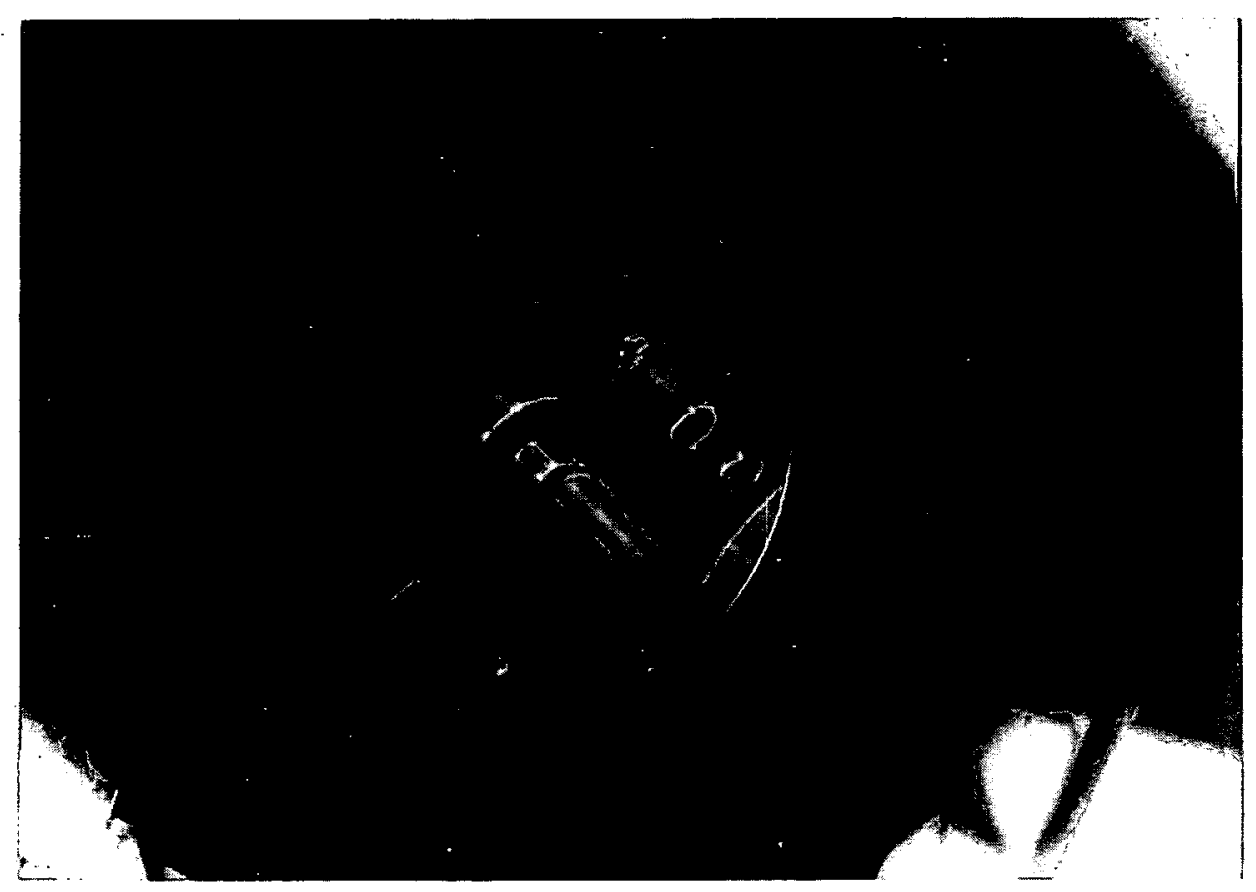

Fig. 4-11. Close-up of coring tool in operation 


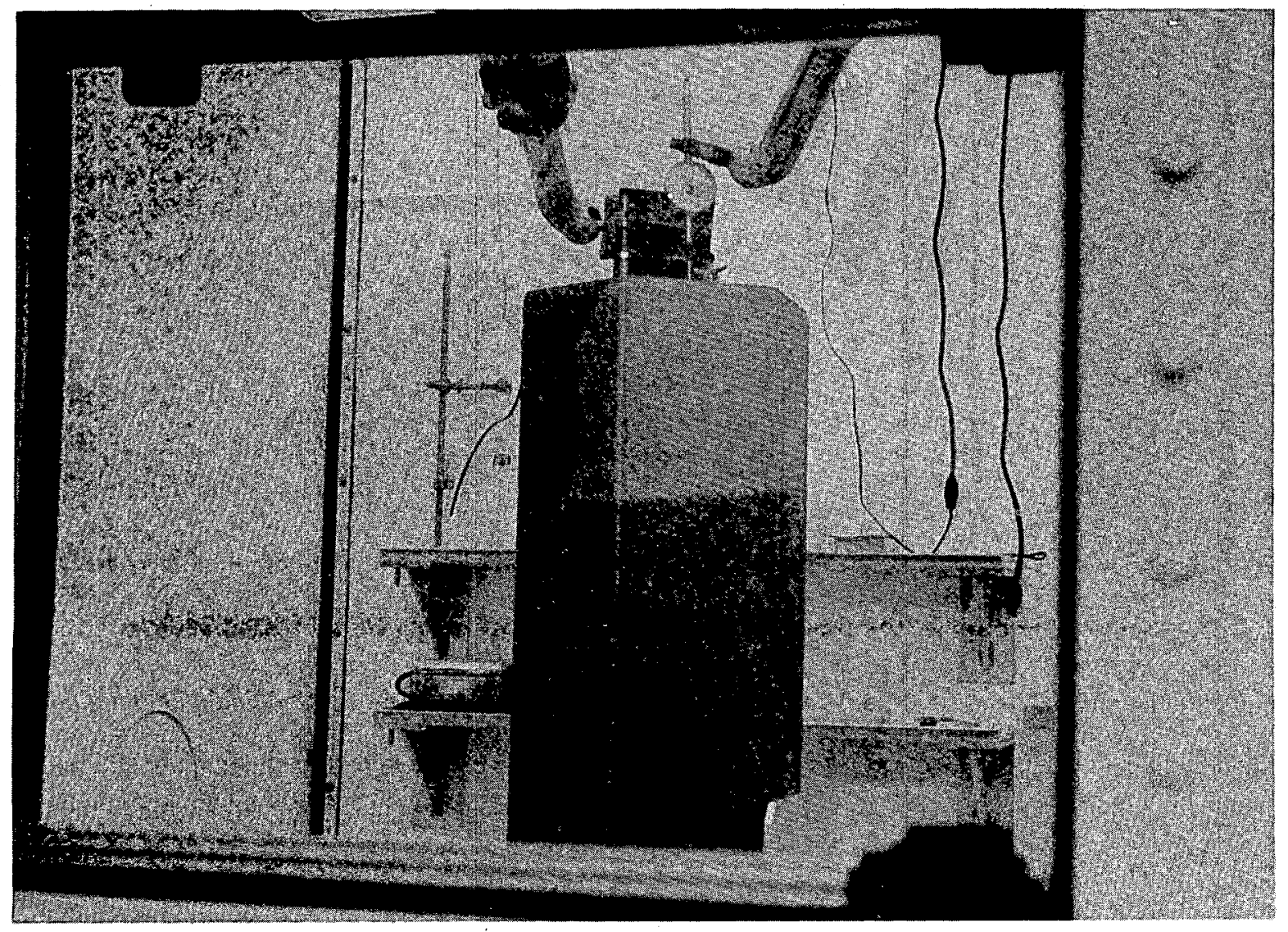

Fig. 4-12. Plenum depth measurement at top surface of fuel element 


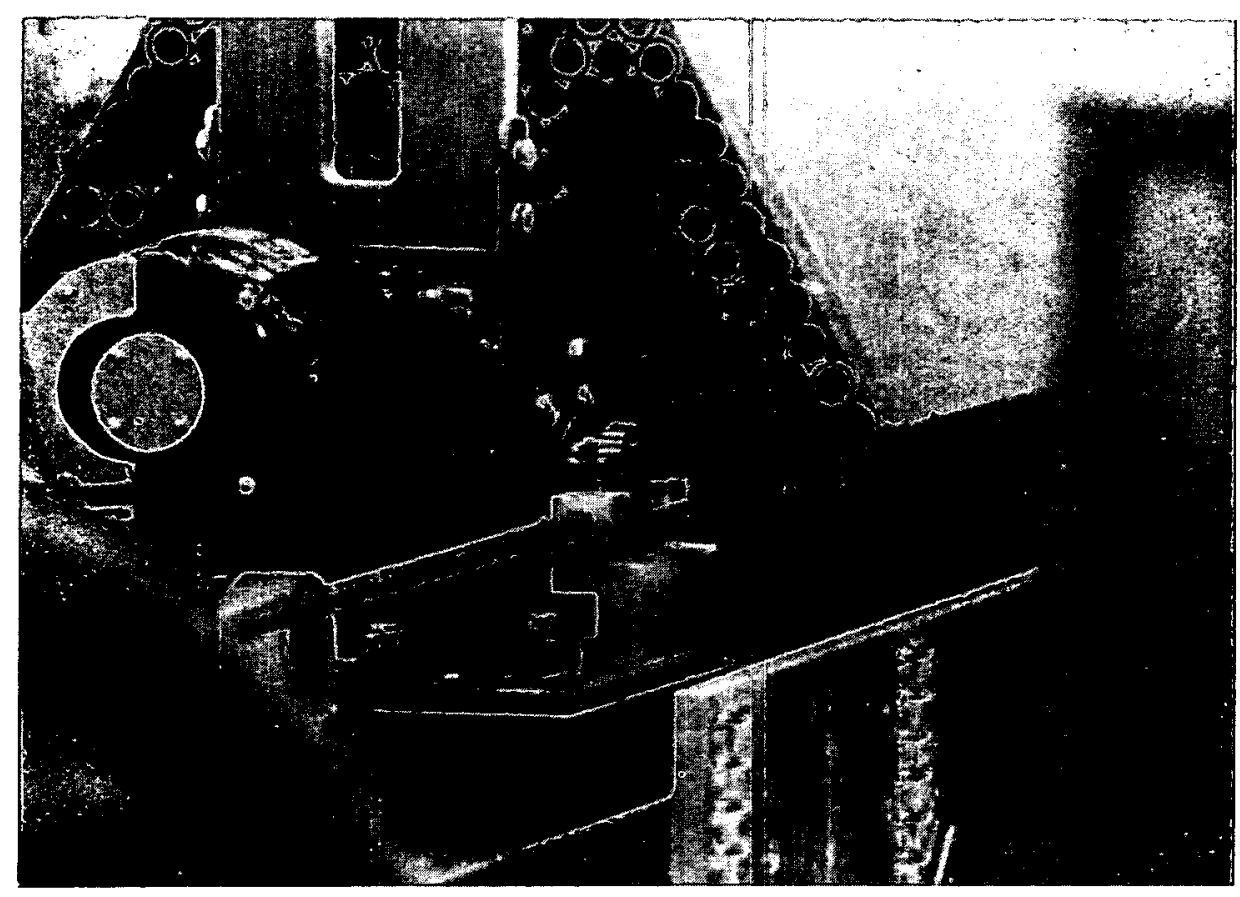

Fig. 4-13. Push-out drive in operation

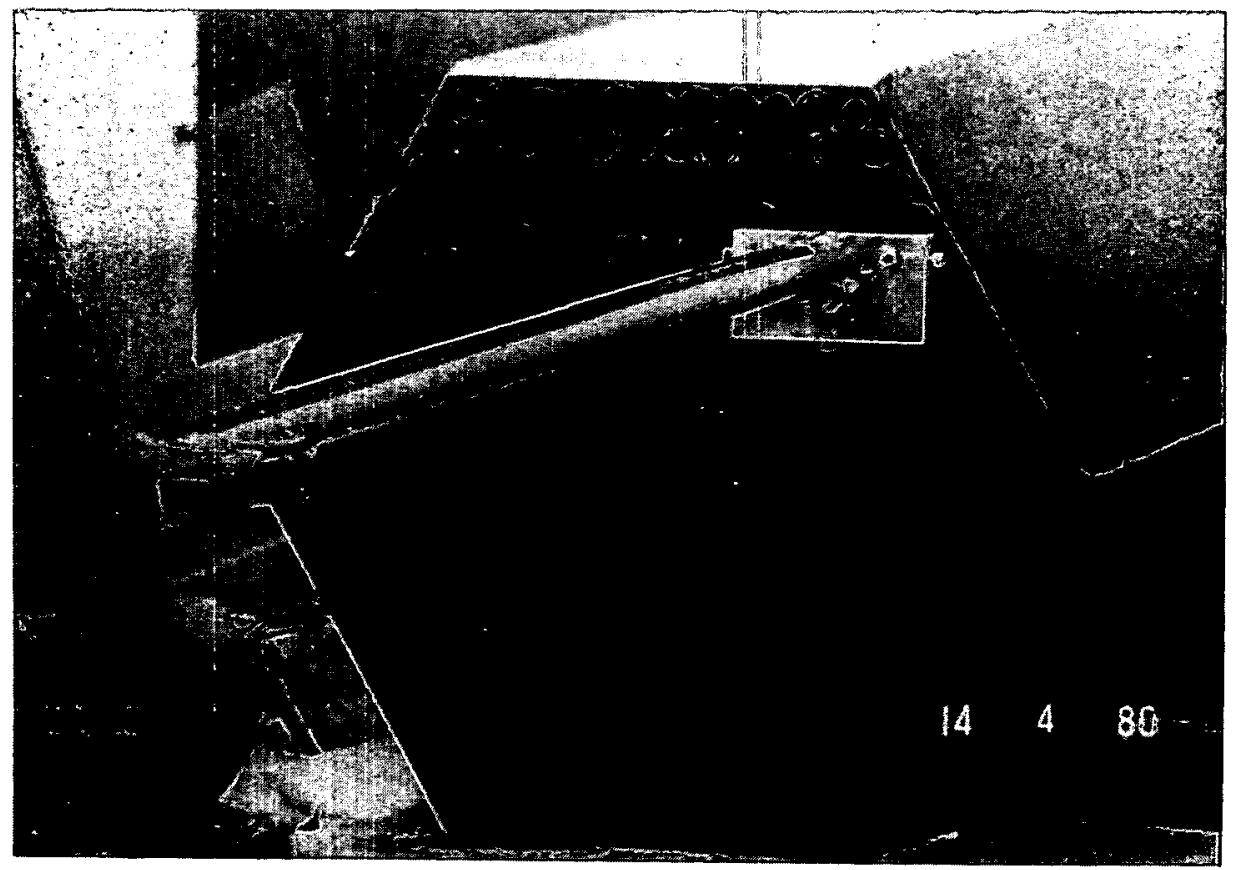

Fig. 4-14. Dual-tube receiving trough for fuel rod stacks 



Fig. 4-15. Fuel rods irradiated in FSV fuel element 1-0743. Chipping and end cap cracking observed at ends of rods: (a) rod 12-2, end of rod is chipped (S8020-1); (b) rod 278-13: cracks in matrix end cap (S8020-34); rod 47-14: chipping at end of rod, failed fuel particles observed ( $58020-77)$. 

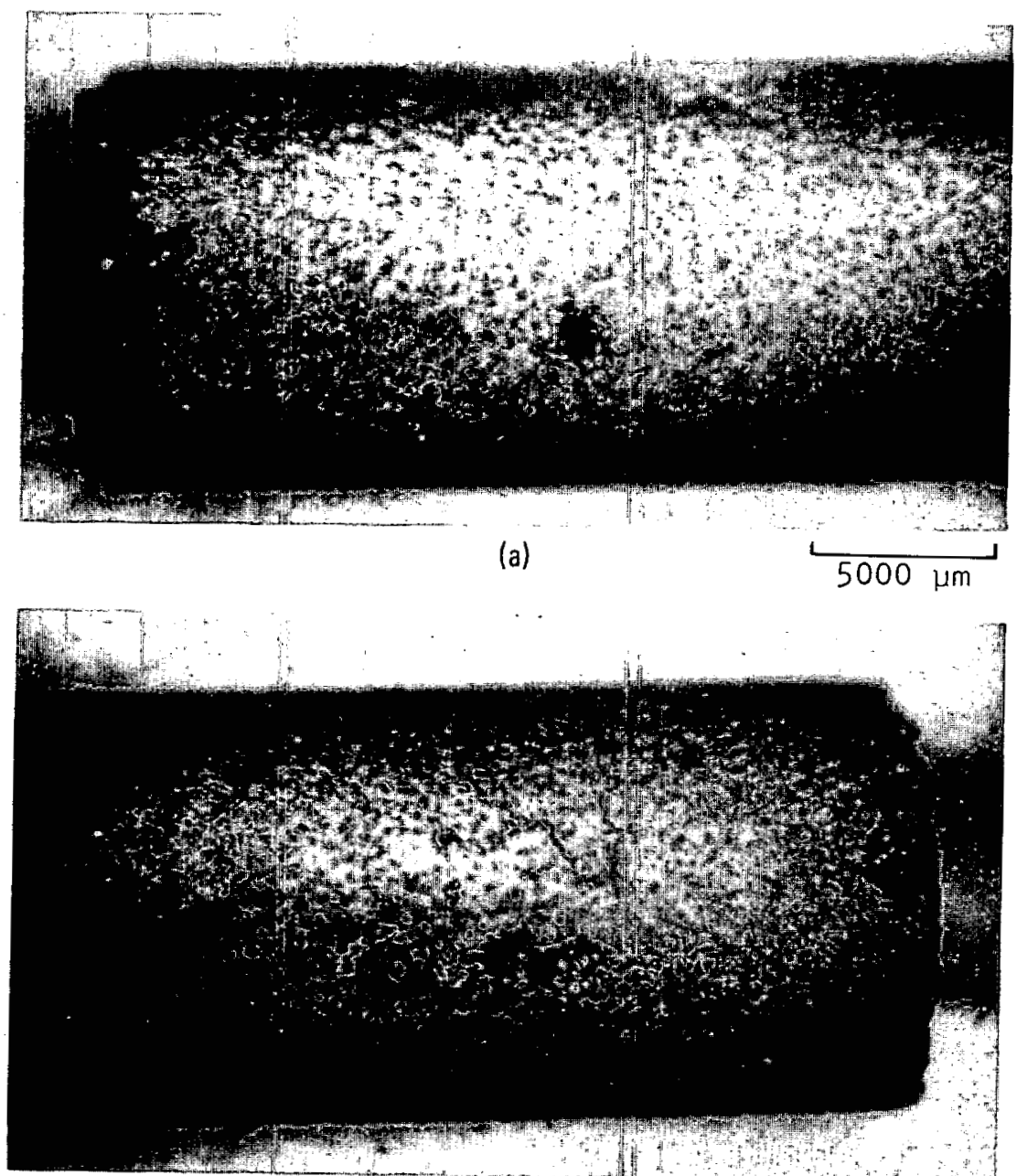

(b)

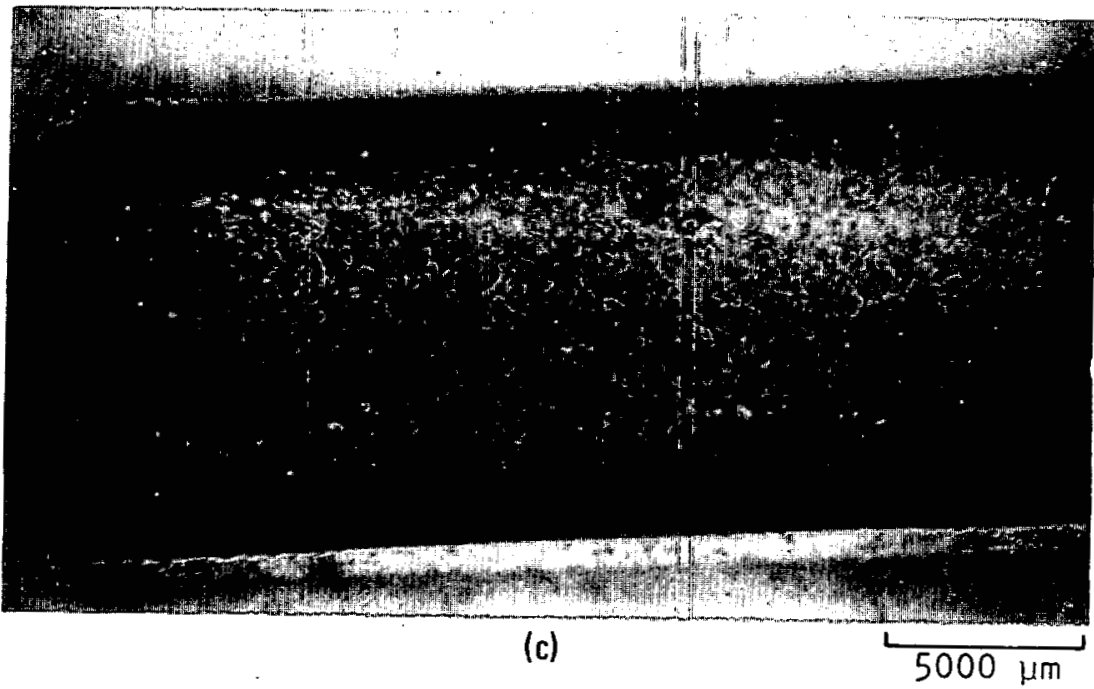

Fig. 4-16. Fuel rods irradiated in FSV fuel element 1-0743. Debonding observed on surfaces of rods: (a) rod 278-2 (S8020-20); (b) rod 278-8 (S8020-27); (c) rod 189-7 (S8020-56); striation resulting from interaction of loose particles or graphite debris (from coring operation) with fuel rod during removal from element also observed. 

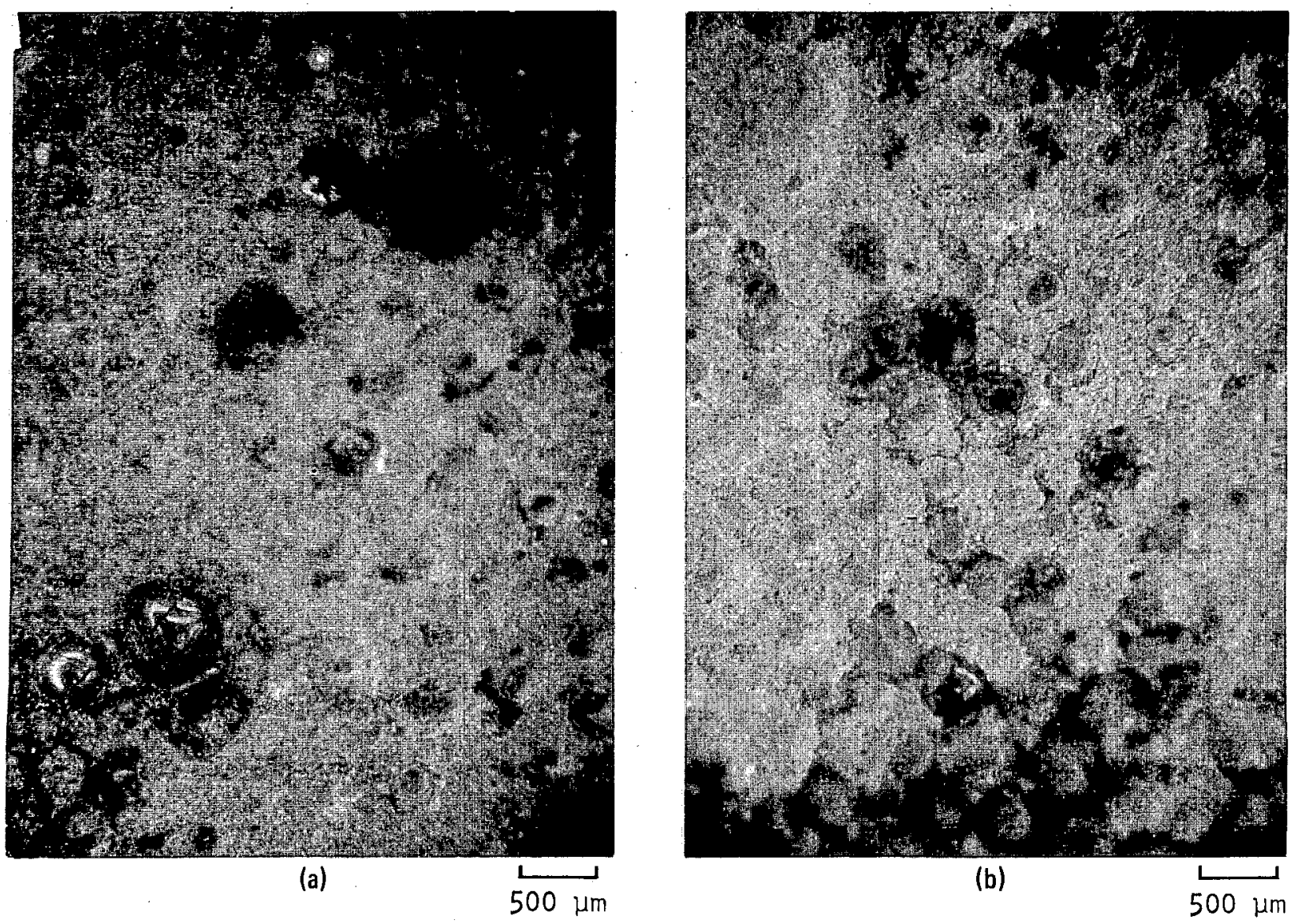

Fig. 4-17. Fuel rods irradiated in FSV fuel element 1-0743. Broken particles observed on end surfaces of fuel rods: (a) rod 12-2 $(\mathrm{S} 8020-8)$; (b) $\operatorname{rod} 12-7(\mathrm{~S} 8020-11)$. 


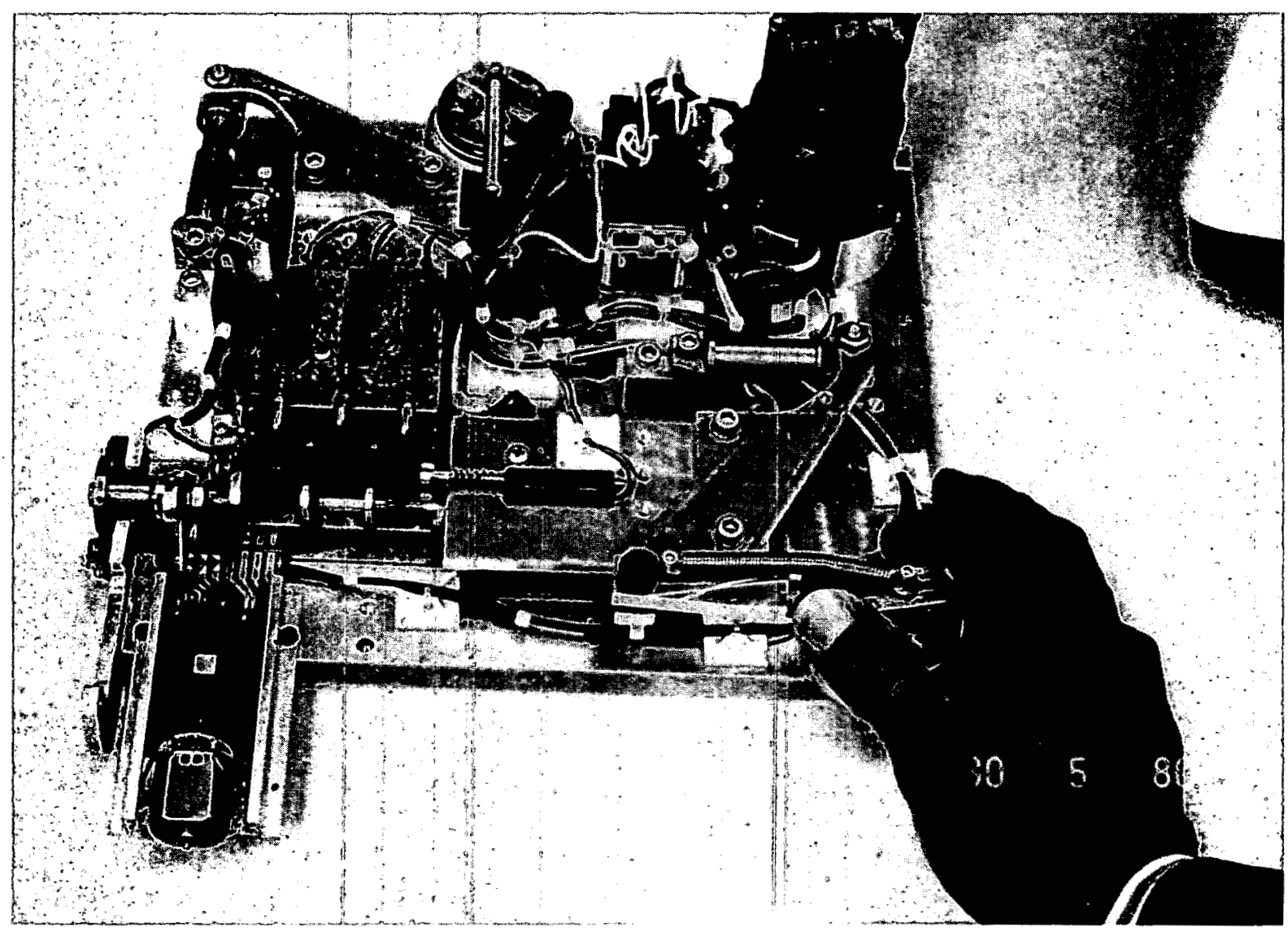

Fig. 4-18. Automated fuel rod dimensioning device used for metrology of fuel rods irradiated in FSV fuel element 1-0743 


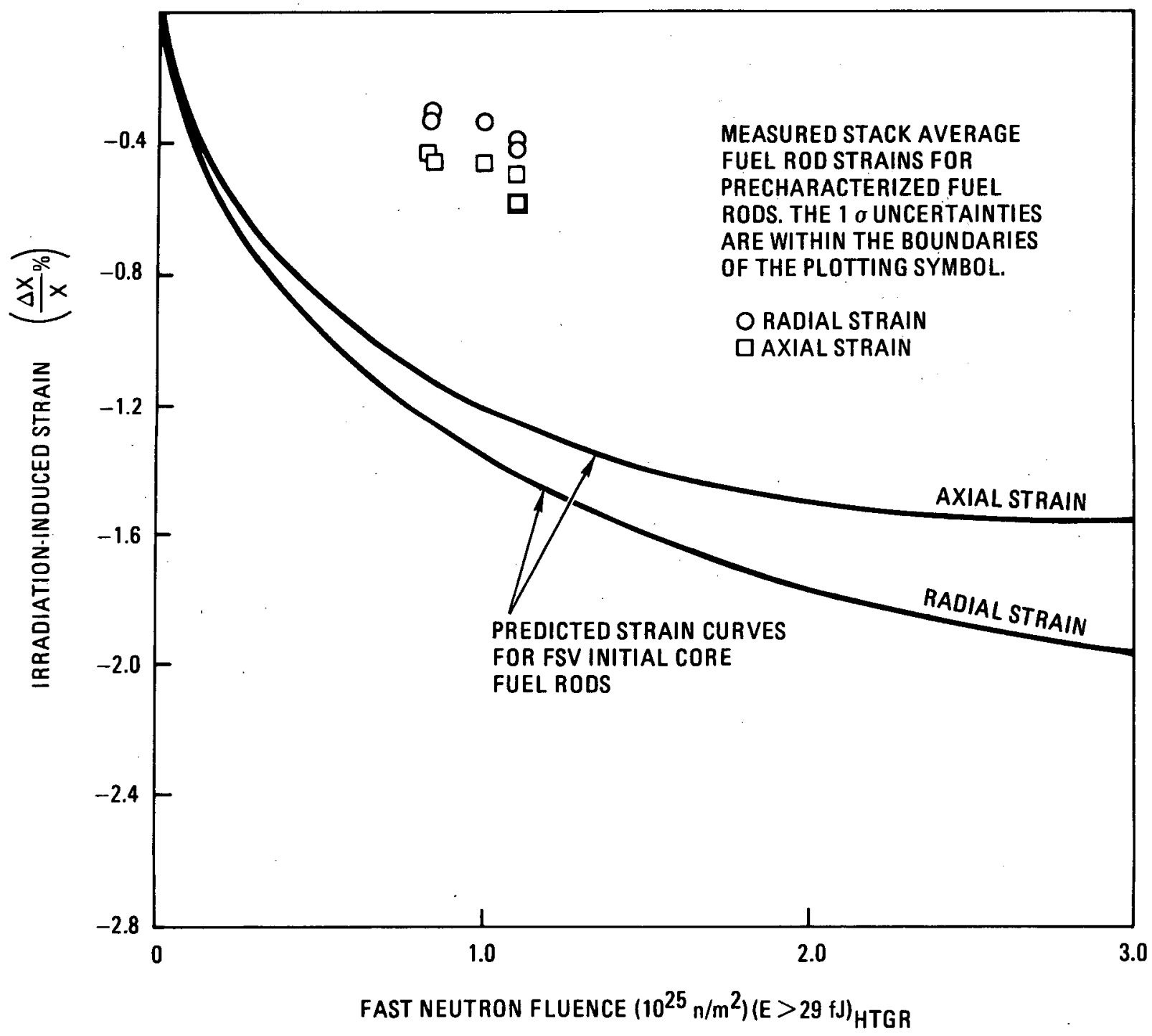

Fig. 4-19. Comparison of calculated and measured strain for fuel rods irradiated in FSV fuel element 1-0743 


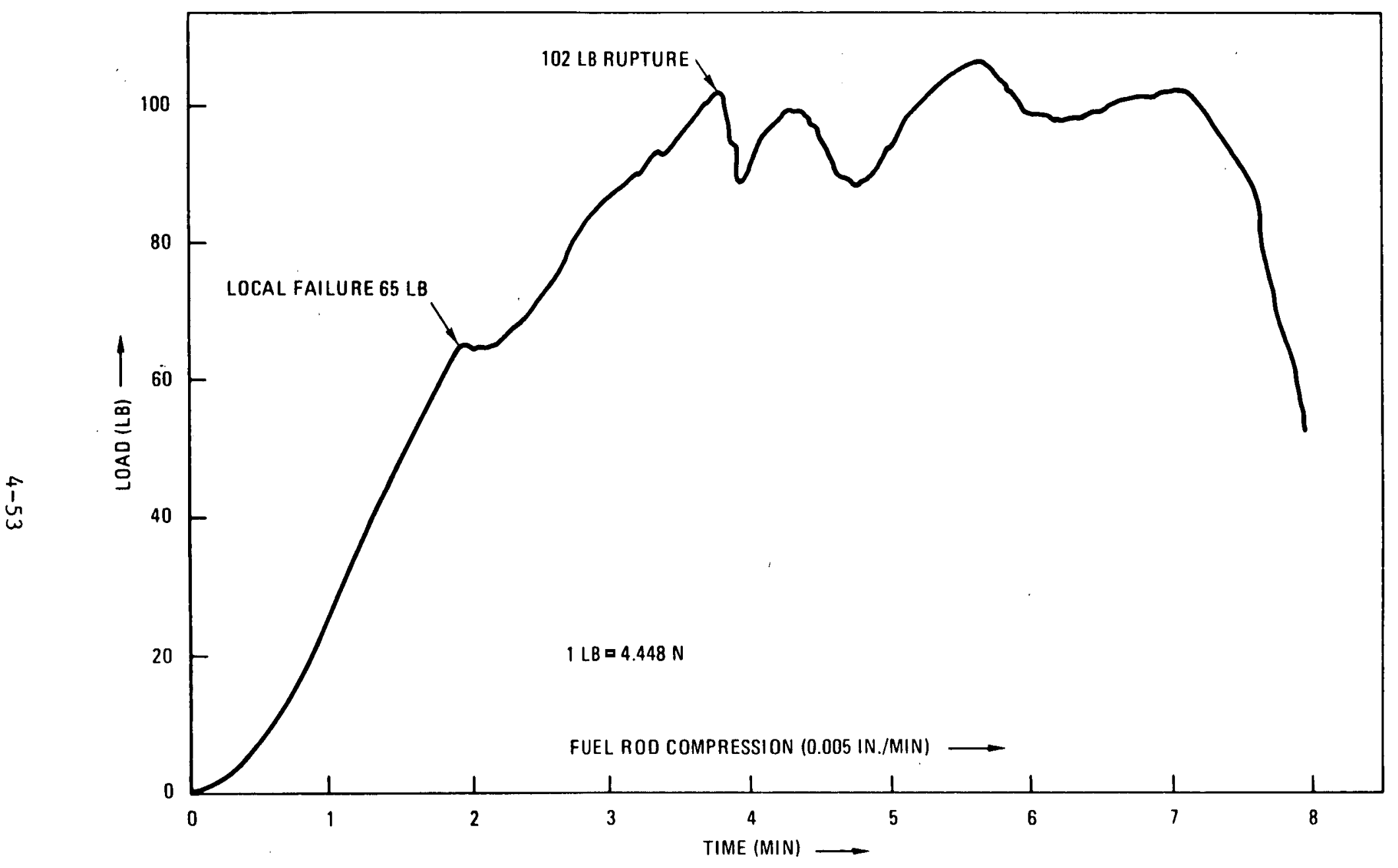

Fig. 4-20. Load distribution during strength testing of a typical irradiated fuel rod from FSV fuel element 1-0743 

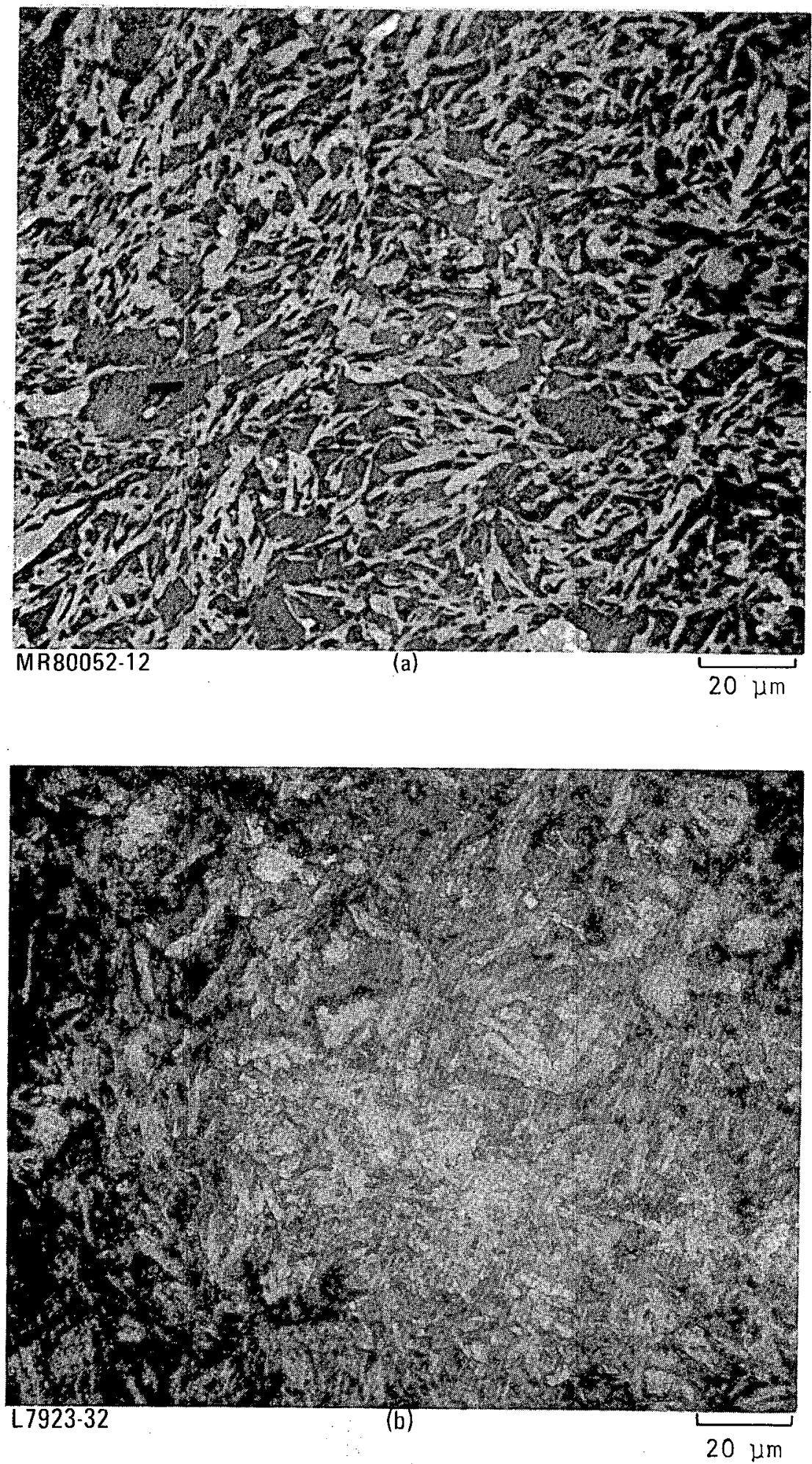

Fig. 4-21. Photomicrographs representative of matrix phase: (a) unirradiated; (b) irradiated in FSV fuel element $1-0743$ at $720^{\circ} \mathrm{C}$ to a fluence of $1.0 \times 10^{25} \mathrm{n} / \mathrm{m}^{2}$. (E $>29 \mathrm{fJ}$ ) HTGR. The matrix phase is difficult to distinguish in (b) because the polished section was etched. 


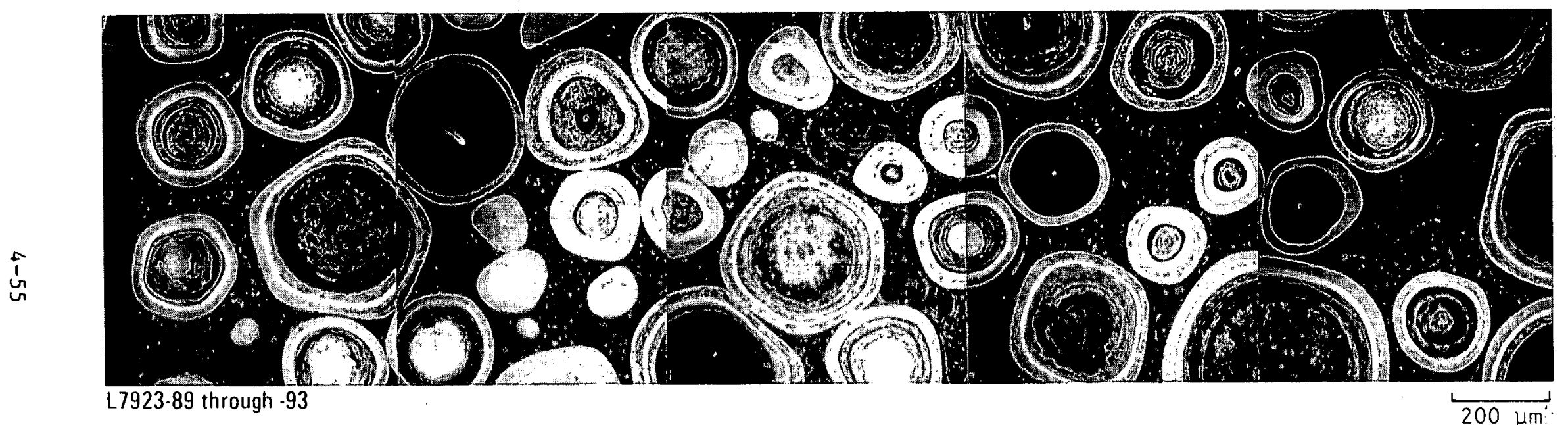

Fig. 4-22. Representative photomicrograph of composite of radial cross section of fuel rod irradiated in FSV fuel element $1-0743$ at $705^{\circ} \mathrm{C}$ to a fluence of $1.1 \times 10^{25} \mathrm{n} / \mathrm{m}^{2}$ (E $\left.>29 \mathrm{fJ}\right){ }_{\mathrm{HTCR}}$. The matrix phase is difficult to distinguish because the polished section was etched. 

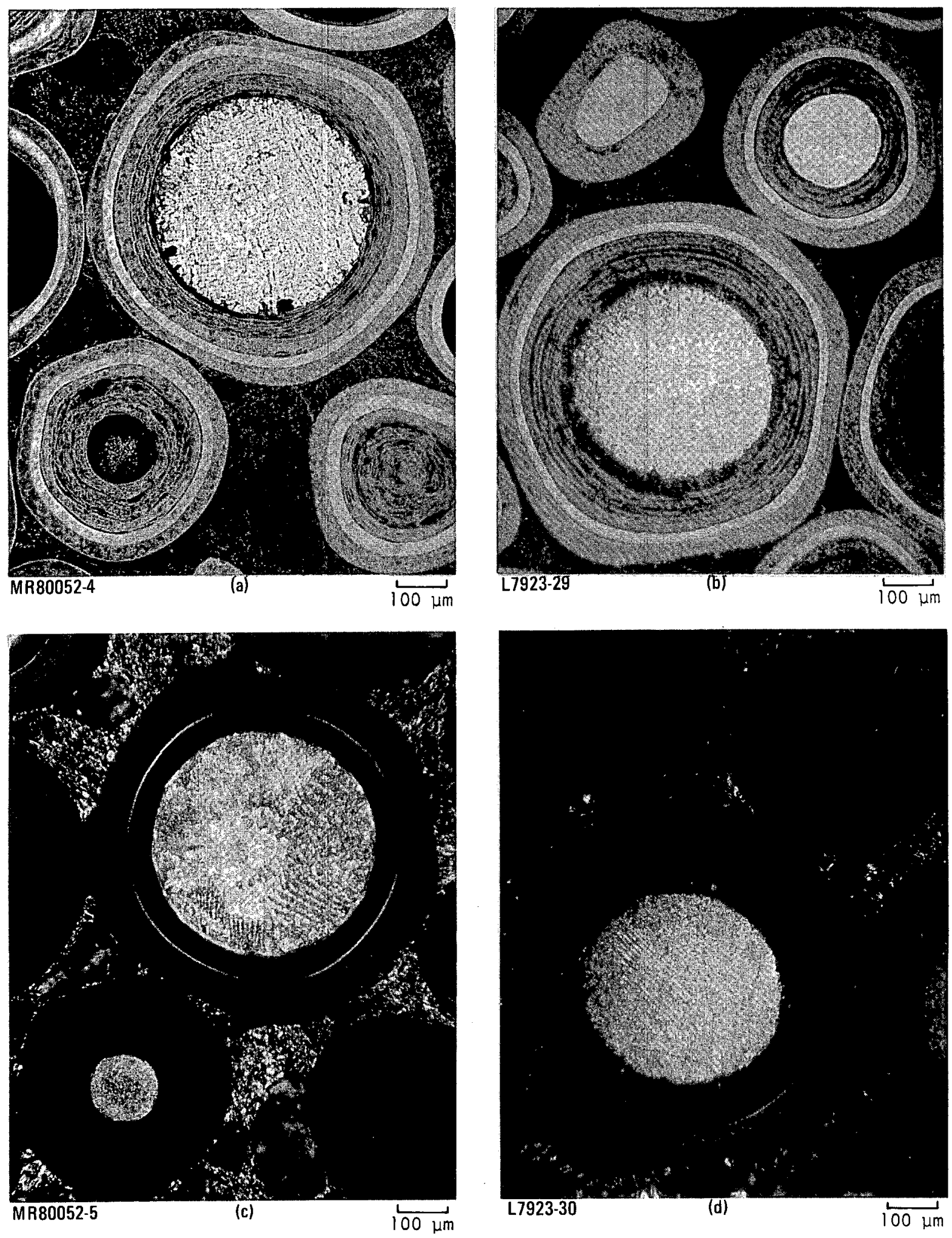

Fig. 4-23. Photomicrographs of fissile (small) and fertile (large) particles: (a) and (c) unirradiated; (b) and (d) irradiated in FSV fuel element 1-0743 to a fluence of $1.0 \times 10^{25} \mathrm{n} / \mathrm{m}^{2}(\mathrm{E}>29 \mathrm{fJ})_{\mathrm{HTGR}}$ at a temperature of $720^{\circ} \mathrm{C}$. (a) and (b) bright field illumination; (c) and (d) polarized light. 

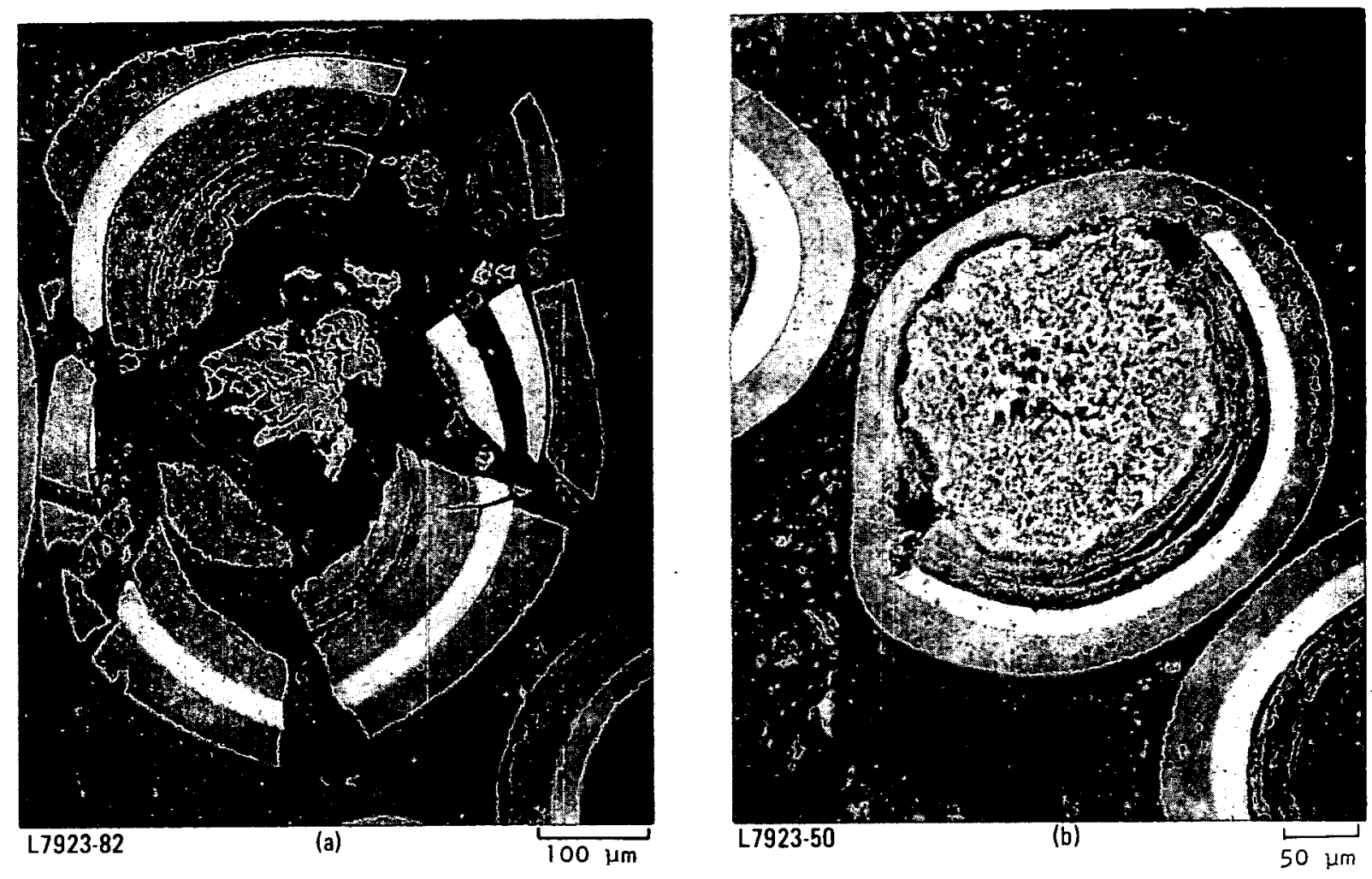

Fig. 4-24. Photomicrographs of examples of as-manufactured defective particles in irradiated fuel rods: (a) total coating failure, (b) Sic coating failure 

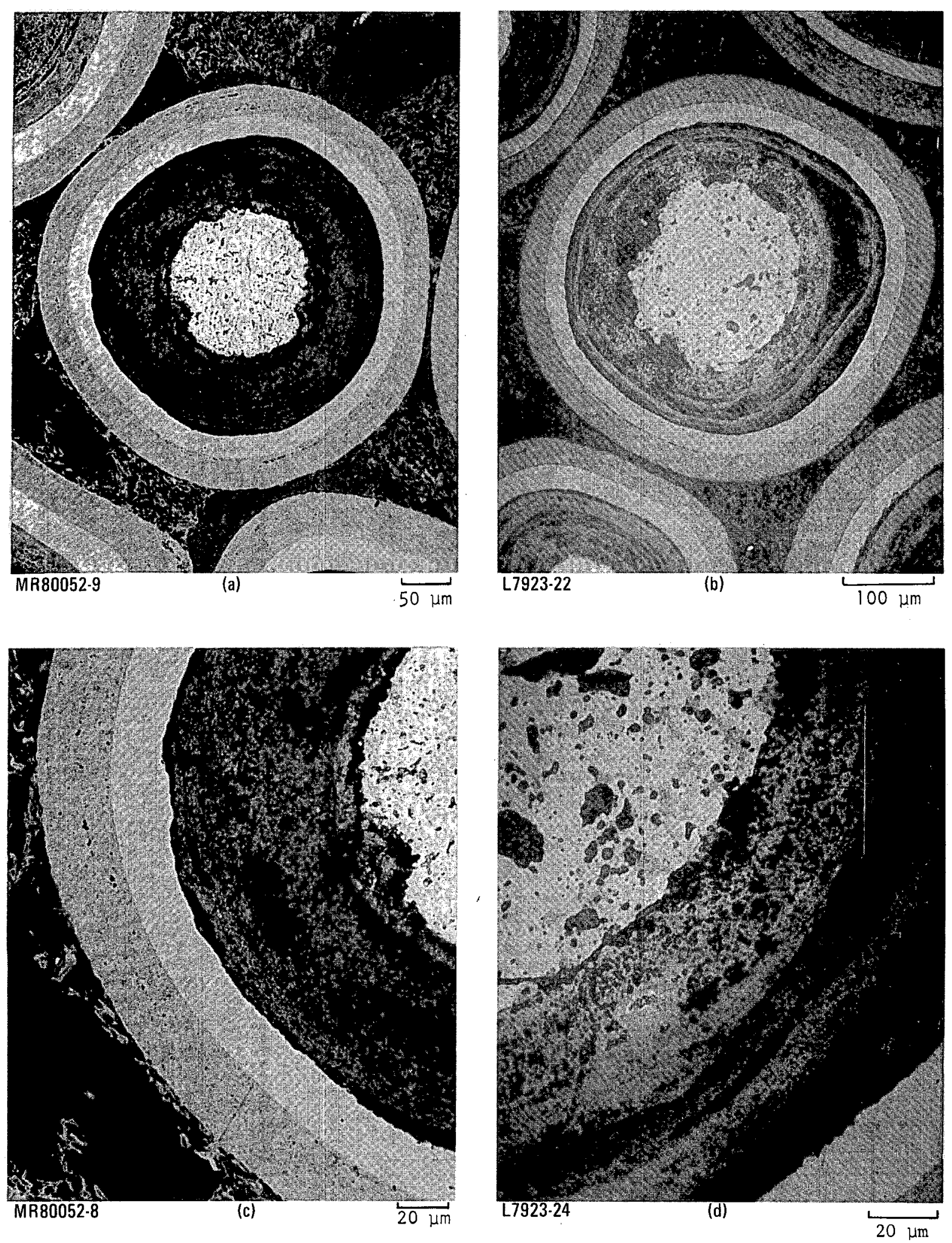

Fig. 4-25. Photomicrographs of TRISO (Th,U) $C_{2}$ particles apparently exhlbiting slight fuel dispersion in buffer coating: (a) and (c) unirradiated; (b) and (d) irradiated in FSV fuel element $1-0743$ at $720^{\circ} \mathrm{C}$ to a burnup of $6.1 \%$ FIMA. Note the low-density IPyC coating. 


\section{SUMMARY AND CONCLUSIONS}

FSV fuel element 1-0743 was irradiated for 174 EFPD in core location 17.04.F.06, experiencing an average fast neutron exposure of about $0.95 \mathrm{x}$ $10^{25} \mathrm{n} / \mathrm{m}^{2}$ (E > $29 \mathrm{fJ}$ ) HTGR, a time- and volume-averaged fuel temperature in the vicinity of $680^{\circ} \mathrm{C}$, fissile and fertile fuel particle burnups of about $6.2 \%$ and $0.3 \%$ FIMA, respectively, and a total burnup of $12,210 \mathrm{MWd} /$ tonne. The element was removed from the reactor during the first refueling in February 1979. After undergoing nondestructive examination in the hot service facility at FSV in July 1979, the element was shipped to the GA hot cell for extensive PIE.

The PIEs of fuel element 1-0743 at FSV and at GA were performed as part of the DOE-sponsored surveillance program for FSV. The purpose of these examinations was to verify the good performance of the fuel element and to acquire in-pile data for verification of core design methods. In addition, the examination of the element at GA was designed to verify the techniques developed for nondestructive examination of core components in the hot service facility at FSV. The results of the PIEs of fuel element 1-0743 are summarized below.

\subsection{FUEL ELEMENT PERFORMANCE}

The performance of the fuel element was excellent. Specific observations are as follows:

1. The graphite fuel body was in good condition. No cracks were observed on any of the surfaces. All observed blemishes were surface markings only and had not etched the graphite to a harmful extent. 
2. The graphite fuel block was dimensionally stáble. The average shrinkage in the block was only $1.3 \mathrm{~mm}$ in length and $0.5 \mathrm{~mm}$ across flats. The maximum observed bow was only $0.3 \mathrm{~mm}$.

3. No evidence of mechanical interaction between the fuel rods and fuel body was found. A clearance of at least $37 \mathrm{~mm}$ was observed between the top fuel rod and the fuel hole plug in six fuel holes for which plenum depth measurements were made. Except in a few cases, very little force was required to push the fuel rods out of the block. Misalignment of the fuel rod receiving trough, and debris from the coring and removal of the fuel hole plugs and graphite containment are believed to be causes of the occasionally high push-out forces.

4. Although minor cracking in the matrix end caps and some surface debonding were observed, the fuel rods were in good condition. No more than 21 broken fuel particles were observed on the surface of any rod. About 3\% of the rods were broken, but the majority were broken during unloading, and the evidence indicates that the remainder were broken prior to assembly of the element.

5. Irradiation-induced dimensional changes in the fuel rods were small and slightly anisotropic. The average radial and axial strains were $-0.36 \%$ and $-0.49 \%$, respectively. The matrix porosity, which is composed of voids $\geq 50 \mu \mathrm{m}$, increased from $19 \%$ prior to irradiation to $26 \%$ after irradiation.

6. The fuel rod compressive strength increased by approximately $15 \%$ as a result of irradiation.

7. The results of fission gas release measurements and metallography indicate no in-pile fuel failure. Approximately 1500 fissile and 925 fertile particles were examined during metallography. For the $(\mathrm{Th}, \mathrm{U}) \mathrm{C}_{2}$ and $\mathrm{ThC}_{2}$ particles, respectively, the OPyC coating 
failure was $0.5 \%$ and $1.1 \%$, the SiC coating failure $0.7 \%$ and $0.5 \%$, and the total coating failure $0.3 \%$ and $0.2 \%$. However, the evidence indicates that the failed coatings were as-manufactured failures which occurred during coating or fuel rod fabrication.

8. The chemical behavior of the particles was acceptable. No chemical attack on SiC coatings was observed, and no kernel migration was seen. A small amount of a dense phase, attributed to fuel dispersion in as-manufactured particles, was observed in the buffer coating of some $(\mathrm{Th}, \mathrm{U}) \mathrm{C}_{2}$ particles. The fuel dispersion did not detrimentally affect the performance of the particles.

\subsection{VERIFICATION OF HTGR CORE DESIGN METHODS}

HTGR design codes used to calculate irradiation and performance parameters for fuel element 1-0743 are summarized below:

GAUGE :

column average power, neutron flux, and nuclide inventories. Radial power distributions, neutron fluences, and fuel burnup can be obtained from GAUGE output using the appropriate axial distributions obtained from another source. Two GAUGE analyses were performed for FSV cycle 1, a "detailed" GAUGE for which the power history was represented by 335 time intervals, and a "short" GAUGE for which the power history was represented by only 11 time intervals.

FEVER:

axial power, neutron flux, and nuclide inventory distributions.

BUG-2 : axial power, neutron flux, and nuclide inventory distributions for fuel elements influenced by control rods in neighboring elements. 
GATT :

axial and radial power distributions, neutron fluence, and fuel burnup.

SURVEY: $\quad$ temperatures and fuel performance. SURVEY also calculates neutron fluences and fuel burnup by bringing together GAUGE, FEVER, and BUG-2 results. SURVEY analysis for FSV cycle 1 is based on the "detailed" GAUGE .

SURVEY/STRESS: stresses, strains, and deformation for the graphite fuel body.

Verification of HTGR core design methods cannot be accomplished from comparisons of experimental observations and design code calculations for one element. Instead, many such comparisons for core components which have collectively experienced a wide range of irradiation conditions are required. One of the primary objectives of the FSV surveillance program is to provide the in-pile data required for these comparisons. The results of comparisons between measurements and design code calculations for fuel element 1-0743 should be reviewed with this in mind. The results are as follows :

1. Radial power distribution: The observed tilt in the time-averaged power distribution was $9 \%$ (relative to element average power), and the calculated tilts were $13 \%$ from SURVEY-detailed GAUGE and $4 \%$ from the short GAUGE. At EOL, the observed tilt was $8 \%$ and calculated tilts were $4 \%$ from SURVEY-detailed GAUGE, $3 \%$ from GATT, and $4 \%$ from the short GAUGE. The agreement between calculated and measured local to block average power factors was within $7.5 \%$ for all local points. This is well within the $\pm 10 \%$ ( $1 \sigma)$ uncertainty for GAUGE calculations. 
2. Axial power distribution: At EOL, the agreement between calculated and measured local to block average power factors was within about $3 \%$ at all axial positions. The time-averaged distributions were also in good agreement except near the bottom of the block, where the axial power was underpredicted by about $10 \%$. The reason for this discrepancy is that the FEVER model cannot account for the control rod in region 34, which was partially inserted during much of cycle 1 . The effect of this control rod was to tilt the axial power toward the bottom of the element.

3. Neutron fluences: The agreement between measured and calculated fast fluences was within $6 \%$ for all comparisons. Calculated fluences were obtained from SURVEY-detailed GAUGE, GATT, and short GAUGE-GATT. The predicted thermal fluence (from short GAUGE-GATT) is $11.9 \%$ smaller than the thermal fluence determined from V-Co dosimeters and $39.9 \%$ greater than the fluence determined from pure $\mathrm{V}$ dosimeters. The fluence determined from the $\mathrm{V}$ dosimeters is believed to be in error.

4. Temperature: The calculated temperature for each temperature monitor was approximately $25^{\circ} \mathrm{C}$ greater than the measured temperature. In all cases, the calculated temperature was within the $95 \%$ confidence limits for the measured temperature.

5. Fuel burnup: The relative differences between measured and calculated composite burnups (indicative of total power generation) were $3.5 \% \pm 2.0 \%(1 \sigma)$ for SURVEY-detailed GAUGE, $9.9 \% \pm 1.9 \%$

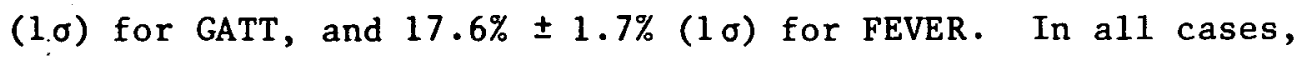
calculated burnups were less than measured burnups. The fissile particle burnup was slightly better predicted than the fertile burnup. 
6. Isotopic composition: The atom \% concentrations of $\mathrm{U}-234, \mathrm{U}-235$, $\mathrm{U}-236$, and $\mathrm{U}-238$ in the $\mathrm{UC}_{2}$ particles irradiated in the burnup monitors were measured and calculated. The relative differences in the measured and calculated atom \% concentrations are $0.4 \% \pm$ $0.2 \%(1 \sigma)$ for $U-234,3.7 \% \pm 0.0 \%$ for $U-235,18.9 \% \pm 0.2 \%(1 \sigma)$ for $\mathrm{U}-236$, and $10.5 \% \pm 0.1 \%(1 \sigma)$ for $U-238$. The concentrations of U-234 and U-235 were overpredicted; the concentrations of U-236 and U-238 were underpredicted.

7. Fuel body strain ( $\mathrm{H}-327$ graphite): A comparison of measured and calculated strains and bow for all 49 segment 1 fuel elements examined at FSV is presented in Ref. 1.

8. Fuel rod strain: The radial strain was predicted to be approximately $1.3 \%$, but strains of only about $0.4 \%$ were measured. Axial strains were also overpredicted by about a factor of 3 . One possible explanation is that the model used to predict the strain was developed primarily from design data in the fast fluence range 4 to $10 \times 10^{25} \mathrm{n} / \mathrm{m}^{2}$ ( $\mathrm{E}>29 \mathrm{fJ}$ ) HTGR and extrapolated to low fluence. This extrapolation may have introduced some error into the model.

9. Fuel performance: In-pile failure was calculated to be $0.32 \%$ for the $(\mathrm{Th}, \mathrm{U}) \mathrm{C}_{2}$ fissile particles and $0.07 \%$ for the $\mathrm{ThC}_{2}$ fertile particles. These failures were attributed to manufacturing defects. The conclusion from the fuel rod examination was that no in-pile failure occurred. The model for failure due to manufacturing defects therefore appears to be conservative. 


\subsection{VERIFICATION OF NONDESTRUCTIVE EXAMINATION TECHNIQUES}

- Techniques for performing visual, metrological, and gamma spectroscopic examinations of core components in the hot service facility at FSV using automated data acquisition systems were verified. The results are as follows :

1. A visual examination of the fuel block was performed in the hot cell. Nothing of significance was observed that had not been observed during the earlier examination at FSV using the metrology robot TV camera system.

2. In order to verify the results of the metrological examination performed at FSV using the metrology robot, the metrological examination was repeated at GA using conventional hot ce11 measuring techniques. A comparison of the results of these measurements with the results obtained with the metrology robot, and comparisons of robot measurements and QC measurements on a calibration fuel block established that the accuracy of the metrology robot is $\pm 0.18 \mathrm{~mm}(0.007 \mathrm{in.})(1 \sigma)$ or better for each type of robot measurement after corrections are applied for observed measurement biases.

3. The element average composite burnups determined from gamma scanning and from destructive measurements agreed to within $2.8 \% \pm 2.1 \%(1 \sigma)$.

4. The gamma scan robot currently being developed for gamma scanning core components at FSV was successfully employed (in a preliminary state of development) to examine fuel element 1-0743 in the hot cel1 at GA. 
- 


\section{ACKNOWLEDGMENTS}

The authors wish to acknowledge the following contributors to this report:

Preparation and Preirradiation Characterization of Element

W. W. Hudritsch, W. J. Scheffel, and O. M. Stansfield

Element Irradiation

Courtesy of Public Services of Colorado (PSC)

Nuclear, Thermal, and Strain Calculations

V. Malakhof, S. C. Bachelor, M. Y. Wan, and W. Shen

Data Reduction

F. McCord

Data Acquisition at Hot Cell

H. O. Johnson and staff, D. W. Hill, F. McCord, T. L. Smith, and

W. E. Simpson

Analysis of Gamma Scan Data

R. C. Jensen (San Diego State University)

Burnup Analysis

T. B. Crockett, D. M. Fleishman, and M. A. Hiatt

Fission Gas Release Measurements

R. W. Tomlin

Analysis of SiC Temperature Monitors

P. W. Flynn and R. G. Hudson

Editing/Typing

J. E. Weaver and staff 
- 


\section{REFERENCES}

1. Miller, C. M., and J. J. Saurwein, "Non-destructive Examination of 51 Fuel and Reflector Elements from Fort St. Vrain Core Segment 1," DOE Report GA-Al6000, General Atomic Company, to be published.

2. Wagner, M. R., "GAUGE, A Two-Dimensional Few Group Neutron Diffusion-Depletion Program for a Uniform Triangular Mesh," USAEC Report GA-8307, General Atomic Company, March 15, 1968.

3. Kraetsch, H., and M. R. Wagner, "GATT, A Three-Dimensional Few Group Neutron Diffusion Theory Program for a Hexagonal-z Mesh," USAEC Report GA-8547, Genera1 Atomic Company, January 1, 1969.

4. Todt, F. W., and L. J. Todt, "FEVER/M1, A One-Dimensional Depletion Program for Reactor Fuel Cycle Analysis," General Atomic Report GA-9780, October 22, 1969.

5. Dorsey, J. P., R. Froehlich, and F. Todt, "BUG-2/BUGTRI, TwoDimensional Multigroup Burnup Codes for Rectangular and Hexagonal Geometry," USAEC Report GA-8272, General Atomic Company, August 22, 1969 .

6. Georghiou, D. L., "SURVEY, A Computer Code for the Thermal and Fuel Performance Analysis of High-Temperature Gas-Cooled Reactors," General Atomic Company, unpublished data, November 1978.

7. Smith, P. D., "SURVEY/STRESS, A Model to Calculate Irradiation-Induced Stresses, Strains, and Deformations in an HTGR Fue1 Block Using Viscoelastic Beam Theory," General Atomic Report GA-Al3712, October 20, 1975 .

8. Malakhof, V., "GATT, Analysis of FSV Cycle 1," General Atomic Company, unpublished data, August 1979.

9. Malakhof, V., "Analytical Results for FSV Surveillance Element 17.04.F.06," General Atomic Company, unpublished data, July 25, 1980. 
10. Saurwein, J. J., and C. F. Wallroth, "Nuclear and Thermal Design Verification for the Peach Bottom High-Temperature Gas-Cooled Reactor," DOE Report GA-A14726, General Atomic Company, September 1979.

11. Boden, R. J., "Fue1 Rod Homogeneity - FSV Segments 1, 7, and 8," General Atomic Company, unpublished data, April 22, 1980.

12. Mathews, D., and V. Malakhof, "FSV Dosimetry Cross Sections," Genera1 Atomic Company, unpub1ished data, September 15, 1980.

13. Palentine, J. E., "The Development of Silicon Carbide as a Routine Irradiation Temperature Monitor and Its Calibration in a Thermal Reactor," J. lJuc1. Mater. 61, 243-253 (1976).

14. Peterson, J. F., "TAC2D, A General Purpose Two-Dimensional Heat Transfer Computer Code," USAEC Report GA-8868, General Atomic Company, September 6, 1969 .

15. Miller, C. M., "Test Specification for Metrology of FSV Graphite Fue1 Elements," General Atomic Company, unpublished data, June 10, 1980.

16. McCord, F., and T. L. Smith, "Test Procedure for Metrology of Irradiated FSV Fuel Rods using the Fuel Rod Measuring Device (MICROBOT)," General Atomic Company, unpublished data, Apri1 30, 1980.

17. Jameson, L., "Bias for the Fort St. Vrain Preirradiation Fuel Rod Diameter Measurements," General Atomic Company, unpublished data, July 16,1980 .

18. Kovacs, W. J., and D. P. Harmon, "Irradiation-Induced Dimensiona1 Changes in HTGR Fue1 Rods," General Atomic Company, unpublished data, October 1979 .

19. "Fuel Design Data Manua1," Issue C, General Atomic Company, unpublished data.

20. Scott, C. B., and D. P. Harmon, "Postirradiation Examination of Capsule F-30," General Atomic Report GA-A13208, Apri1 1, 1975.

21. Stansfield, 0. M., "Thermally Induced Fuel Dispersion in Production Fuel Particles," General Atomic Company, unpublished data, October 14, 1971 .

22. Stansfield, 0. M., "Additional Discussion of Thermally Induced Fuel Dispersion in Production Fuel Particles," General Atomic Company, unpublished data, January 27, 1972. 
APPENDIX A

DISCUSSION OF BURNUP ANALYSIS

by

T. B. Crockett

A-1 


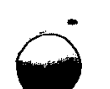


Three monitor packages removed from Stacks 12 and 278 of FSV-Surveillance Element No. 1 contained fissile particles which were analyzed in accordance with procedure ACD;RC-001, "Atom Percent Fission in Fissile and Fertile Fuel Particles." Since these monitor packages had not been designed to incorporate fertile particles, the fertile particles we did use for assay had to be selected from fuel rods. We separated the $T h / U$ fissile and Th fertile particles based on Cs-134/Cs-137 end-of-life ratio. After selection of the Th fertile particles an abbreviated burnup analysis was performed rather than that specified in ACD:RC-001.

The fissile fuel particles were cleaned to remove external contamination, and after this cleaning operation each particle was measured for prominent fission products. Fission product ratios were calculated for each sample to reveal any abnormal fuel particles, i.e., either damaged or particles foreign to set being analyzed.

The ASTM radiochemical method was used in the analysis of the fissile fue 1 particles. This method uses fission product $\mathrm{Cs}_{\mathrm{S}}-137$ as burnup monitor. In addition to the fission product method, the fissile fuel particles were analyzed by a mass spectrometric uranium isotopic analysis method. This method measures burnup through changes in uranium isotopic composition and can be applied only to fuel particles that do not contain thorium or U-233 before irradiation; thus it is not applicable for fertile fuel particles.

Replicate analyses were preformed on the fissile particles passing the selection criteria. Initially, the particles were crushed and dissolved in perchloric acid mixture. These solutions containing fission products and uranium were separated by an anion exchange method. A portion of the $U$ fraction from each of the samples was analyzed mass spectrometrically for both uranium concentration and uranium isotopic composition. Results from isotope dilution mass spectrometric analyses 
are compared with colorimetric results in Table 1; and the fissile fuel particle atom percent uranium isotopic composition results (both archive and irradiated) are in the attached report.

The mass spectrometric data from the LFE report was treated in accordance with ASTM procedure E244, "Atom Percent Fission in Uranium and Plutonium Fue 1 (Mass Spectrometric Method)." Burnup determined by this method is shown in the attached computer printout. Table 2 provides a comparison of mass spectrometric fissile burnup with that measured radiochemically.

Fertile burnup analysis by the abbreviated case basically took advantage of the fact that due to elapsed time since end of irradiation, no $\mathrm{Pa}-233 \mathrm{ac}-$ tivity remained in these fertile particles. We then proceeded to irradiate (in TRIGA) these particles along with bare kernel $\mathrm{ThO}_{2}$ standards and generated $\mathrm{Pa}-233$ activity. By virtue of the $\mu \mathrm{Ci} \mathrm{Pa}-233 / \mathrm{mgm} \mathrm{Th}$ in the bare kernels, we computed the Th weight in the FSV particles on the basis of their respective Pa-233 activities. Lie made an estimate of the end-of-life $U$ in these particles by comparing fission product Ce 143 in the FSV fertile particles with that produced in some bare kernels enriched $\mathrm{UO}_{2}$ particles. After consideration of the differences between U-233 and U-235 fission cross-sections and fission product yields plus estimating $U-233$ to be $85-90 \%$ of the final end-of-life $U$ the overall error is roughly $20 \%$. This has little effect upon the final FIMA values since the $U$ represents only $1: 3 \%$ of end-of-life heavy metal content. The fertile FIMA's shown in Table 3 were computed by the following equation:

$$
F_{3}=\frac{F}{T h^{0}} \times 100=\frac{F}{T h^{R}+-U^{R}+F} \times 100=\% \text { FIMA }
$$

where:

$F_{3}=$ Heavy element atom percent fission from $U-233$ (Th-232).

$F=$ Fissions per total sample $=N^{\prime} / Y$.

$N^{\prime}=$ Atoms of $\mathrm{Cs}-137$ (corrected for decay during and after irradiation).

$Y=$ Fractional fission yields of Cs-137 (6.80\%).

$\mathrm{Th}^{\mathrm{O}}=$ Initial atoms of thorium.

$U^{R}=$ Remaining atoms of uranium.

$\mathrm{Th}^{\mathrm{R}}=$ Remaining atoms of thorium.

One last item worth noting is that the ASTM Method generates a flux value based on the isotopic composition change. I have underlined those values on the attached computer printout. The fission to capture value for $U-235(.2238)$ was obtained from the materials you originally provided.
cc:
D. $\mathrm{Hill}$
D. Flieshman
M. Hiatt 
TABLE 1

\begin{tabular}{|c|c|c|c|}
\hline $\begin{array}{l}\text { SAMPLE } \\
\text { MONI TOR } \\
\end{array}$ & $\begin{array}{l}\text { IDENTITY } \\
\text { PARTICLEE }\end{array}$ & $\begin{array}{l}\text { MASS SPEC* } \\
\cup \mu G M .\end{array}$ & $\begin{array}{l}\text { CHEMISTRY* } \\
\cup \mu G M\end{array}$ \\
\hline 21 & 4 & 10.93 & 10.69 \\
\hline 21 & 5 & 8.40 & 8.40 \\
\hline 22 & 3 & 9.53 & 9.57 \\
\hline 22 & 4 & 10.12 & 9.81 \\
\hline 81 & 4 & 9.08 & 9.37 \\
\hline 81 & 5 & 10.11 & 9.52 \\
\hline
\end{tabular}

* After chemical yield correction 
TABLE 2

SAMPLE

MONITOR

21

21

22

22

81

81
IDENTITY

PARTICLE

4

5

3

4

4

5
RADIOCHEMISTRY

FIMA

32.1

32.2

31.7

31.6

33.7

31.6
ASTM MASS SPEC

FIMA
30.2

30.8

30.3

30.1

32.8

31.1 
TABLE 3

\section{FERTILE FIMA's}

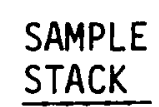

12

12

12

12

12

12

279

279

279

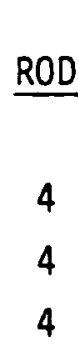

11

11

11

3

3

3
PARTICLE

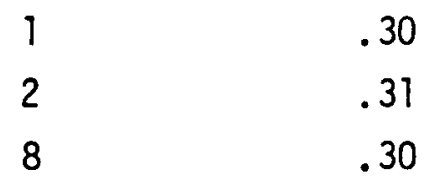

$3 \quad .31$

$4 \quad .32$

$5 \quad .33$

$2 \quad .35$

$6 \quad .33$

$8 \quad .35$ 
MASS SPECTROMETRIC ANALYSIS REPORT

REPORT TO

GENERAL ATOMIC COMPANY

P. 0. BOX. 81608

SAN DIEGO, CALIFORNIA 92138

ORDER NUMBER

BL-81-99 PR-740855

LFE ENVIRONMENTAL

ANALYSIS LABORATORIES DIVISION

2030 WRIGHT AVENUE

RICHMOND, CALIFORNIA 94804

AUGUST 18,1980

PREPARED BY KATSUMI YAMAMOTO, SUPERVISOR

MASS SPECTROMETRY

APPROVED BY R. MELGARD

LABORATORY OPERATIONS MANAGER 
GENERAL ATOMIC COMPANY

BL-81-99 PR-740855

URANIUM ANALYSIS

\begin{tabular}{|c|c|c|c|c|c|c|}
\hline & & & & ATOM & RCENT & \\
\hline & Monitor & Part & 234 & 235 & 236 & 238 \\
\hline B3 & 21 & $\$ 4$ & $\begin{array}{r}0.800 \\
\pm 0.004\end{array}$ & $\begin{array}{l}79.93 \\
\pm 0.03\end{array}$ & $\begin{array}{l}10.80 \\
\pm 0.03\end{array}$ & $\begin{array}{l}8.475 \\
\pm 0.021\end{array}$ \\
\hline B4 & 21 & $\# 5$ & $\begin{array}{r}0.795 \\
\pm 0.003\end{array}$ & $\begin{array}{l}79.87 \\
\pm 0.04\end{array}$ & $\begin{array}{l}10.79 \\
\pm 0.04\end{array}$ & $\begin{array}{l}8.548 \\
\pm 0.022\end{array}$ \\
\hline B5 & 22 & $\# 3$ & $\begin{array}{r}0.795 \\
\pm 0.007\end{array}$ & $\begin{array}{l}79.92 \\
\pm 0.05\end{array}$ & $\begin{array}{l}10.80 \\
\pm 0.04\end{array}$ & $\begin{array}{r}8.48 \\
\pm 0.04\end{array}$ \\
\hline B6 & 22 & \#4 & $\begin{array}{r}0.792 \\
\pm 0.007\end{array}$ & $\begin{array}{l}79.97 \\
\pm 0.04\end{array}$ & $\begin{array}{l}10.788 \\
\pm 0.025\end{array}$ & $\begin{array}{r}8.46 \\
\pm 0.03\end{array}$ \\
\hline B7 & 81 & $\# 4$ & $\begin{array}{r}0.797 \\
\pm 0.005\end{array}$ & $\begin{array}{l}79.29 \\
\pm 0.05\end{array}$ & $\begin{array}{l}11.10 \\
\pm 0.04\end{array}$ & $\begin{array}{l} \pm 8.81 \\
\pm 0.04\end{array}$ \\
\hline B8 & 81 & $\$ 5$ & $\begin{array}{l}0.7979 \\
\pm 0.0016\end{array}$ & $\begin{array}{l}79.39 \\
\pm 0.06\end{array}$ & $\begin{array}{l}11.23 \\
\pm 0.06\end{array}$ & $\begin{array}{l}8.582 \\
\pm 0.027\end{array}$ \\
\hline
\end{tabular}

PARTS PER MILLION

B3

B4

B5

B6

B7

B8

\begin{tabular}{rrrr}
\multicolumn{1}{c}{235} & \multicolumn{2}{c}{236} & \multicolumn{2}{c}{238} & TOTAL \\
0.775 & 0.1052 & 0.0832 & 0.971 \\
\pm 0.008 & \pm 0.0011 & \pm 0.0009 & \pm 0.010 \\
0.592 & 0.0803 & 0.0641 & 0.742 \\
\pm 0.006 & \pm 0.0009 & \pm 0.0007 & \pm 0.008 \\
0.675 & 0.0916 & 0.0726 & 0.846 \\
\pm 0.007 & \pm 0.0011 & \pm 0.0009 & \pm 0.009 \\
0.718 & 0.0973 & 0.0769 & 0.899 \\
\pm 0.008 & \pm 0.0011 & \pm 0.0009 & \pm 0.010 \\
0.638 & 0.0898 & 0.0718 & 0.806 \\
\pm 0.007 & \pm 0.0010 & \pm 0.0009 & \pm 0.009 \\
0.712 & 0.1012 & 0.0779 & 0.898 \\
\pm 0.008 & \pm 0.0013 & \pm 0.0009 & \pm 0.010
\end{tabular}


